\title{
A Synaptic and Systems Level Investigation of Memory Processes Using a Novel Multiple Memory Behavioural Procedure
}

\section{Brianne C. Wartman}

A thesis submitted to the Faculty of Graduate and Postdoctoral Affairs in partial fulfillment of the requirements for the degree of

Doctor of Philosophy

in

Neuroscience

Carleton University

Ottawa, ON

(C) 2014

Brianne C. Wartman 


\section{$0.1 \quad$ Abstract}

It is proposed that memories stabilize through synaptic and systems level consolidation processes, forming long-lasting memory representations. Systems consolidation models suggest that hippocampal contribution to memory storage disengages as time progresses, while ensembles of cortical neurons are proposed to form increasingly strong connections that store and represent remote memories as consolidation proceeds. Investigations into memory representations highlight discrepancies between the involvement of a cortical region in remote memory processes, the anterior cingulate cortex (ACC), and the hippocampus. The present thesis introduces a novel behavioural procedure that examines the effect of increased processing demands on the hippocampus and the consequent effect on memory consolidation, storage and retrieval.

Rats were trained on a single spatial task, two different spatial tasks, or one spatial and one non-spatial task. Regional involvement of the hippocampus and the ACC during retrieval were examined using immediate early genes and proteins of interest. Structural modifications in memory storage were examined using the Golgi-Cox method and quantification of dendritic complexity was analyzed through neuron reconstruction. The contribution of the ACC to spatial memory retrieval was assessed through behavioural performance and transient ACC pharmacological inactivation.

The present thesis provides evidence that increased demand on the hippocampus results in accelerated processing of spatial memories. Findings show that the involvement of the ACC in memory processing can be manipulated by increasing hippocampal-processing demand. Functional, structural and behavioral data suggest that taxing the demand on the hippocampus accelerates the involvement of the ACC. There is also evidence of continued hippocampal involvement in memory processes at recent and remote time points. Taken together, these findings indicate an increased recruitment of the ACC, but not an accelerated independence from the hippocampus, in spatial memory processes. The use of a novel behavioral procedure aimed at increasing hippocampaldemand can reveal processes more similar to those present in the human condition, where there is a constant demand on memory systems. The novelty of the findings presented in this thesis will further our understanding of remote memory. 


\subsection{Co-Authors}

Mathew R. Holahan

Jennifer Gabel 


\subsection{Acknowledgments}

There are many people to credit with helping me reach this goal. I owe thanks

to my colleagues, the staff and faculty at Carleton University and my supervisor, Dr. Matthew Holahan. I want to extend a special thanks to my friends and family for their encouragement and patience with me throughout this process and the reassuring words they provided me with countless times. 


\subsection{Dedication}

I dedicate this thesis to my soon-to-be husband, Dr. Zack Patterson.

Over the last five years, Zack has had unwavering confidence in my abilities.

He has celebrated my successes and supported me through tough times. I could not have done this without him.

Zack, I am so proud that we have made it through this process together. I owe this success to you. 


\subsection{List of Abbreviations}

ACC, anterior cingulate cortex; aCSF, artificial cerebral spinal fluid; AMPAR, $\alpha$-amino-3-hydroxy-5-methyl-4-isoxazolepropionic acid receptor; Arc, activityregulated cytoskeleton-associated protein; Arg, activity-regulated gene; BME, beta mercaptoenthanol; CA, cornu ammonis; CaMKII, calcium /calmodulindependent protein kinase II; CMT, cognitive map theory; DG, dentate gyrus; E-LTP, early long-term potentiation; EC, entorhinal cortex; fMRI, functional magnetic resonance imaging; GAP-43, growth-and plasticity-associated protein; GAPDH, Glyceraldehyde 3-phosphate dehydrogenase; GluR2, glutamate receptor 2; HPC, hippocampus; IDV, integrated density value; IEG, immediate early gene; L-LTP, late long-term potentiation; L, left; LTP, long-term potentiation; mPFC, medial prefrontal cortex; mRNA, messenger ribonucleic acid; MTL, medial temporal lobe; MTT, multiple trace theory; NMDAR, NMethyl-D-aspartic acid receptor; NSF, N-ethylmaleimide-sensitive factor; ODN, oligodeoxynucleotide; OP, operant conditioning; PDK1, phosphoinositide- dependent protein kinase 1; PFC, prefrontal cortex; PKA, protein kinase A; $\mathrm{PKC} \zeta$, protein kinase $\mathrm{C} \zeta$; PSD, postsynaptic density; PVDF, polyvinylidene difluoride; $\mathrm{R}$, right; $\mathrm{RA}$, retrograde amnesia; RAM, radial arm maze; RIPA, Radio Immuno-Precipitation Assay; RNA, ribonucleic acid; SB, subiculum; SMC, standard model of consolidation; TGRA, temporally graded retrograde amnesia; WM, water maze 


\section{Contents}

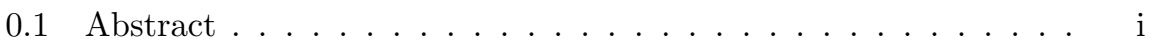

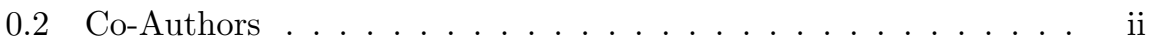

0.3 Acknowledgments . . . . . . . . . . . . . . iii

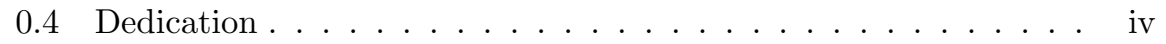

0.5 List of Abbreviations . . . . . . . . . . . . . . $\mathrm{v}$

List of Figures $\quad$ X

List of Tables xii

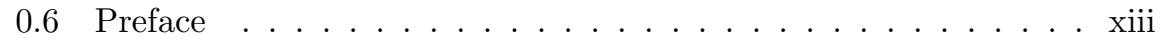

1 Introduction $\mathbf{1}$

1.1 General Introduction . . . . . . . . . . . . . . . . . 1

1.2 Where is Memory Stored in the Brain? . . . . . . . . . . . . 3

1.3 The Hippocampus . . . . . . . . . . . . . . . . . 4

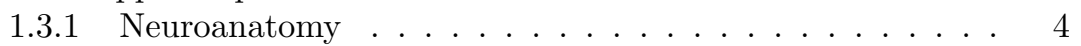

1.3.2 Memory .................. 5

1.3.3 Spatial Function ................ 6

1.4 The Anterior Cingulate Cortex . . . . . . . . . . . . . 8

1.4.1 Neuroanatomy .................. 9

1.4.2 Connections between the Hippocampus and Anterior Cingulate Cortex . . . . . . . . . . . . . . . 9

1.5 Behavioural Tasks ....................... 11

1.5.1 Water Maze . . . . . . . . . . . . . . . . . . 11

1.5.2 Radial Arm Maze .................. . 13

1.5.3 Operant Conditioning .............. . . 14

1.6 Molecular Mechanisms of Memory . . . . . . . . . . . . . . 14

1.6.1 Long-Term Potentiation . . . . . . . . . . . . . . . . 15

1.7 Proteins of Interest in Memory Formation and Storage . . . . . . 17

1.7 .1 c-Fos . . . . . . . . . . . . . . . 17

1.7.2 Activity-regulated cytoskeleton-associated protein . . . . 18

1.7.3 Protein Kinase M $\zeta$. . . . . . . . . . . . . . . . . . . . . . 19

1.7.4 Growth and Plasticity Associated Protein (GAP-43) . . . 21

1.8 Structural Changes Associated with Long-Term Memory . . . . . 21

1.9 Consolidation .................... 24 
1.9.1 Models of Consolidation . . . . . . . . . . . . . 25

1.10 Memory Dynamics . . . . . . . . . . . . . . . . . 27

1.10.1 Roles of the Hippocampus and Prefrontal Cortex . . . . . 27

1.10 .2 Memory Organization . . . . . . . . . . . . . 27

1.10 .3 Limitations . . . . . . . . . . . . . . . . 35

1.11 Objectives \& Hypothesis . . . . . . . . . . . . . . 36

2 The use of sequential hippocampal-dependent and -non-dependent tasks to study the activation profile of the anterior cingulate cortex during recent and remote memory tests 46

2.1 Abstract . . . . . . . . . . . . . . . . 47

2.2 Introduction . . . . . . . . . . . . . . . . . . . 48

2.3 Materials \& Methods . . . . . . . . . . . . . . . . . . . . . . 49

2.3 .1 Subjects . . . . . . . . . . . . . . . . . . 49

2.3.2 Apparatus . . . . . . . . . . . . . . . . . . . . . 49

2.3.3 Behavioural Procedure . . . . . . . . . . . . . . . 50

2.3 .4 Immunohistochemistry . . . . . . . . . . . . . . . . . 51

2.4 Results . . . . . . . . . . . . . . . . . 53

2.4.1 Behavioural Training . . . . . . . . . . . . . . . . 53

2.4.2 Water Maze Probe . . . . . . . . . . . . . . . . . . 53

2.4.3 c-Fos expression in the ACC . . . . . . . . . . 54

2.4.4 c-Fos expression in the CA1 of the hippocampus . . . . 54

2.5 Discussion . . . . . . . . . . . . . . . . . . . 55

2.6 Supplemental Introduction . . . . . . . . . . . . . . . . 58

2.7 Supplemental Materials \& Methods . . . . . . . . . . . . . . . . 59

2.7 .1 Immunohistochemistry . . . . . . . . . . . . . . . 59

2.7 .2 Arc Quantification . . . . . . . . . . . . . . 60

2.8 Supplemental Results . . . . . . . . . . . . . . . . . . 60

$2.8 .1 \quad \mathrm{ACC} \ldots \ldots \ldots \ldots \ldots \ldots$

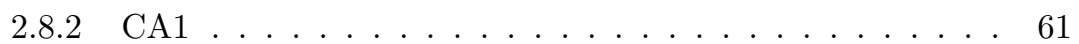

$2.8 .3 \quad \mathrm{CA} 3 \ldots \ldots \ldots \ldots \ldots \ldots$

2.9 Supplemental Discussion . . . . . . . . . . . . . . . . . . . 61

3 The impact of multiple memory formation on dendritic complexity in the hippocampus and anterior cingulate cortex as$\begin{array}{ll}\text { sessed at recent and remote time points. } & \mathbf{7 6}\end{array}$

3.1 Abstract . . . . . . . . . . . . . . . 77

3.2 Introduction . . . . . . . . . . . . . . . . . 78

3.3 Materials \& Methods . . . . . . . . . . . . . . . . . . . . . . 79

3.3.1 Subjects . . . . . . . . . . . . . . . . . . 79

3.3 .2 Apparatus . . . . . . . . . . . . . . . . . . . . 79

3.3.3 Behavioural Procedure . . . . . . . . . . . . . . . . . 80

3.3.4 Golgi-Cox Procedure . . . . . . . . . . . . . . . . . . . . 81

3.3.5 Quantitative neuromorphology . . . . . . . . . . . 82

3.3 .6 Statistical analyses . . . . . . . . . . . . . . . . 83

3.4 Results . . . . . . . . . . . . . . . . . . . . . 83 
3.4.1 Behavioural training . . . . . . . . . . . . . . . . . 83

3.4.2 Neuron morphology . . . . . . . . . . . . . . . . . . . 83

3.5 Discussion . . . . . . . . . . . . . . . . . . . . . . . . 89

3.5.1 Results . . . . . . . . . . . . . . . . 9 90

3.5.2 Conclusion . . . . . . . . . . . . . . . . . . . 93

3.6 Supplemental Introduction . . . . . . . . . . . . . . . . . . 94

3.7 Supplemental Materials \& Methods . . . . . . . . . . . . . . . . . 94

3.7.1 Enzyme-linked immunosorbent assay (ELISA) . . . . . . 94

3.8 Supplemental Results . . . . . . . . . . . . . . . . . . 95

3.9 Supplemental Discussion . . . . . . . . . . . . . . . . . . . . . 95

4 Inactivation of the anterior cingulate reveals enhanced reliance on cortical networks for remote spatial memory retrieval after multiple memory processing

4.1 Abstract . . . . . . . . . . . . . . . . . . 113

4.2 Introduction . . . . . . . . . . . . . . . . . . . . 114

4.3 Materials \& Methods . . . . . . . . . . . . . . . . . . . 115

4.3.1 Subjects . . . . . . . . . . . . . . . . . 115

4.3.2 Surgical Procedures . . . . . . . . . . . . . . . . 115

4.3.3 Apparatus . . . . . . . . . . . . . . . . . . . . 116

4.3.4 Behavioural Procedure . . . . . . . . . . . . . . . . 116

4.3.5 Injections and Water Maze Probe . . . . . . . . . . . . 117

4.3.6 Tissue Processing . . . . . . . . . . . . . . . . . . . . . . 118

$4.3 .7 \quad$ c-Fos quantification . . . . . . . . . . . . . . . . . . . . 1119

4.4 Results . . . . . . . . . . . . . . . . . . . . . . . . . . . . 119

4.4.1 Behavioural Training . . . . . . . . . . . . . . . . . 119

4.4 .2 Water Maze Probe Test . . . . . . . . . . . . . 120

4.4.3 c-Fos labeling in the CA1 of the hippocampus . . . . . . 122

4.4 .4 Placements . . . . . . . . . . . . . . . . . . 123

4.5 Discussion . . . . . . . . . . . . . . . . . . . . . 123

4.6 Supplemental Introduction . . . . . . . . . . . . . . . . . . 126

4.7 Supplemental Materials \& Methods . . . . . . . . . . . . . . . 127

4.7.1 Western Blot . . . . . . . . . . . . . . . . . 127

4.8 Supplemental Results . . . . . . . . . . . . . . . . . . . . . . 128

4.9 Supplemental Discussion . . . . . . . . . . . . . . . . . . . . 128

5 General Discussion $\quad 140$

5.1 Summary of Results . . . . . . . . . . . . . . . . . . . 140

5.2 Consolidation Theories . . . . . . . . . . . . . . . . . . . . 141

5.3 Complementary Memory Systems . . . . . . . . . . . . . . . . . 142

5.4 The Special Case of Spatial Memory . . . . . . . . . . . . . 143

5.5 Relevant Findings and Future Directions . . . . . . . . . . . 144

5.6 Conclusion . . . . . . . . . . . . . . . . . . . . . . . . . 147 
6 Appendix A- Copyright Documentation

6.1 Permission from Neurobiology of Learning and Memory . . . . . 149

6.2 Permission from Frontiers in Behavioural Neuroscience . . . . . . 150

6.3 Co-author Permission Statements . . . . . . . . . . . . . . 151

7 Appendix B-Supervisor Statement 


\section{List of Figures}

1.1 Chapter 1, Figure $1 \ldots \ldots \ldots$

1.2 Chapter 1, Figure $2 \ldots \ldots \ldots \ldots$

1.3 Chapter 1, Figure 3 . . . . . . . . . . . . . . . . . . . . . . . . . 39

1.4 Chapter 1, Figure $4 \ldots \ldots \ldots$

2.1 Chapter 2, Figure 1 . . . . . . . . . . . . . . . . . . . 64

2.2 Chapter 2, Figure $2 \ldots \ldots \ldots \ldots \ldots$

2.3 Chapter 2, Figure $3 \ldots \ldots \ldots$

2.4 Chapter 2, Figure $4 \ldots \ldots \ldots \ldots$

2.5 Chapter 2, Figure $5 \ldots \ldots \ldots \ldots$

2.6 Chapter 2, Figure 6 . . . . . . . . . . . . . . . . . . . . . . . . . . . . 69

2.7 Chapter 2, Figure $7 \ldots \ldots \ldots$

2.8 Chapter 2, Figure $8 \ldots \ldots \ldots \ldots$

2.9 Chapter 2, Figure $9 \ldots \ldots \ldots \ldots$

2.10 Chapter 2, Figure $10 \ldots \ldots \ldots \ldots \ldots$

2.11 Chapter 2, Figure $11 \ldots \ldots \ldots \ldots$

3.1 Chapter 3, Figure 1 . . . . . . . . . . . . . . . . . . . . . 98

3.2 Chapter 3, Figure 2 . . . . . . . . . . . . . . . . . . . . . . . . . . . 99

3.3 Chapter 3, Figure 3 . . . . . . . . . . . . . . . . . . . 100

3.4 Chapter 3, Figure $4 \ldots \ldots \ldots 10 \ldots$

3.5 Chapter 3, Figure 5 . . . . . . . . . . . . . . . . . 102

3.6 Chapter 3, Figure 6 . . . . . . . . . . . . . . . . . . 103

3.7 Chapter 3, Figure 7 . . . . . . . . . . . . . . . . . . . . . . . . 104

3.8 Chapter 3, Figure 8 . . . . . . . . . . . . . . . . . . . . . . . 105

3.9 Chapter 3, Figure 9 . . . . . . . . . . . . . . . . . . . 106

3.10 Chapter 3, Figure $10 \ldots \ldots$. . . . . . . . . . . . . 107

3.11 Chapter 3, Figure 11 . . . . . . . . . . . . . . . . . . . . 108

3.12 Chapter 3, Figure 12 . . . . . . . . . . . . . . . . . . . . . 109

3.13 Chapter 3, Figure 13 . . . . . . . . . . . . . . . . . 110

4.1 Chapter 4, Figure $1 \ldots \ldots \ldots$

4.2 Chapter 4, Figure $2 \ldots \ldots \ldots$

4.3 Chapter 4, Figure 3 . . . . . . . . . . . . . . . . . . . . . 132

4.4 Chapter 4, Figure $4 \ldots \ldots \ldots$ 
4.5 Chapter 4, Figure $5 \ldots \ldots \ldots$

4.6 Chapter 4, Figure $6 \ldots \ldots$. . . . . . . . . . . . . . 135

4.7 Chapter 4, Figure $7 \ldots \ldots \ldots$

4.8 Chapter 4, Figure $8 \ldots \ldots \ldots \ldots \ldots$

4.9 Chapter 4, Figure $9 \ldots \ldots \ldots$

4.10 Chapter 4, Figure $10 \ldots \ldots \ldots$ 


\section{List of Tables}

1.1 Chapter 1 , Table $1 \ldots \ldots$. . . . . . . . . . . . . 41

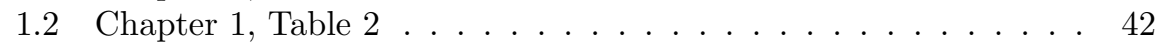

1.3 Chapter 1 , Table 3 . . . . . . . . . . . . . . . . 43

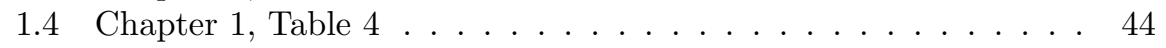




\subsection{Preface}

Memory is an integral part of the human identity. Skills and experiences learned throughout ones life are stored as memories, developing intelligence and creating a sense of self. Memory deficits resulting from disease or injury are often devastating and permanent. The consequences of memory impairments are widespread. The strains placed on caregivers, the large demands on the medical community and the financial burden resulting can be overwhelming. Considering dementias alone, the population afflicted is expected to double, to over 1.1 million in the next 25 years (Rising Tide, Alzheimer Society of Canada, 2010), with an estimated financial burden of 153 billion dollars. Even more devastating than the financial burden is the emotional toll on afflicted individuals and their caregivers. With few effective treatments available to help those suffering from memory impairments, research uncovering the basic mechanisms involved in memory storage must be continued. A more complete understanding of how and where memories are stored in the brain will provide insight on how better to treat those suffering from memory impairments.

The hippocampus has an established role in memory, however, its precise role and involvement in memory processes is controversial. Recently, the anterior cingulate cortex (ACC) has been implicated as an important node in remote memory processes. Conflict exists within and between animal and human research. Humans have a seemingly endless sequence of memory storage and retrieval across the lifetime. Laboratory animals are typically required to store and retrieve only one memory for one task. As a starting point to bridge the human and animal work, it was of interest to develop a behavioural procedure that taxed the memory processing demands of the animal. To this end, a multiple memory behavioural procedure was developed. Briefly, this multiple memory behavioural procedure trained rats on a single spatial task, two different spatial tasks, or, one spatial and one non-spatial task. The multiple memory behavioural procedure examined whether the number of memories processed by an organism affected systems consolidation processes. The present thesis contains three manuscripts that report on the role of the hippocampus and ACC in memory processes by providing behavioural, structural and functional results that shed light on the unsolved quandary of memory storage.

Manuscript 1 (published in Neurobiology of Learning and Memory) introduces a unique behavioural procedure to examine remote memory processes, the multiple memory behavioural procedure described above. Hippocampal and ACC activation were examined. Hippocampal activation was greater when memory retrieval occured at remote, compared to recent, time points. ACC activation was greater remotely, compared to recently, except in rats trained on two spatial tasks. Rats trained on two spatial tasks showed accelerated activation of the ACC at recent time points.

In Manuscript 2 (published in Frontiers in Behavioural Neuroscience) the multiple memory procedure was used for structural analysis of neurons in the hippocampus and ACC. This study was concerned with examining structural changes owing to the consolidation and storage of memories, not their retrieval, 
thus no probe test was administered prior to sacrifice. Structural differences were noted between behavioural groups indicating multiple memory processing led to different morphological modifications. Structural changes indicative of increased dendritic complexity were noted recently in the ACC of rats trained on two spatial tasks, suggesting accelerated recruitment of the ACC in memory processing. Manuscript 2 provides structural support for the functional results presented in Manuscript 1.

Manuscript 3 (submitted to PLOS ONE) directly examined the necessity of the ACC in memory retrieval. In this study, transient inactivation of the ACC revealed a graded effect of behavioural deficits. A role of the ACC in recent memory retrieval was noted in rats trained on two spatial tasks. Manuscript 3 provides direct evidence supporting the activational findings from Manuscript 1 and structural findings from Manuscript 2.

\section{Contrubution of Authors}

Manuscripts 1 and 2 have two authors: the candidate and her supervisor. Manuscript 3 contains an additional author, a summer student, who assisted in conducting some of the behavioural procedures under the supervision of the candidate. The candidate provided the conception and design of the experiments, conducted behavioural testing, provided analysis and interpretation of data, drafted and revised the manuscripts and served as corresponding author dealing with reviewer comments. The supervisor provided guidance in overall design, data analysis and interpretation. The supervisor edited written text, and provided approval for submitted and revised manuscripts. 


\section{Chapter 1}

\section{Introduction}

\subsection{General Introduction}

Learning and memory represent two of the most basic functions characteristic of organisms: the ability to gain, store and access knowledge. Learning and memory are such inherent processes it becomes difficult to define these terms without limiting their function. To empirically study these processes, a working definition is necessary. Learning may be defined as the acquisition of knowledge or skills, which underscores the ability of an organism to change its behaviour (Kandel and Hawkins, 1992). Memory may be defined as the ability to store, retain and recall experiences and information (Kandel and Hawkins, 1992). These two processes are complementary: learning being the process whereby knowledge and information is acquired and memory being the process by which this knowledge is retained and retrieved or used. Learning and memory are often based on changes in synaptic relationships or function at the level of the neuron and inferred from changes in behaviour at the level of the organism.

Human memory is an exquisitely complex process. Memory is defined according to the time since initial acquisition. In this scheme, memories are classified as short-term or long-term. Though no strict distinctions exist, short-term memories are usually defined as those which last for seconds to minutes, while long-term memories are generally defined as persisting for hours to days to years (Atkinson and Shiffrin, 1971, 1968; Kolb and Whishaw, 2011; McGaugh, 2000; Sweatt, 2010). Short-term memory has a finite store (Cowan, 2001; Miller, 1956), while long-term memory is believed to have a limitless capacity whereby an infinite amount of information can be retained on a seemingly permanent basis.

Short-term memory may be subdivided into sensory memory, short-term storage, and working memory (Atkinson and Shiffrin, 1971, 1968; Jeneson and Squire, 2012; Sweatt, 2010; Tulving, 1992). Sensory memory deals with transient information brought in through the senses and represents the initial stage of processing new information into a memory (Sweatt, 1999, 2010). Short-term 
storage involves the retention of information following processing. This processing may include new sensory information, or, the recall of a previously stored memory (Sweatt, 2010). Working memory holds information for short periods of time while it is being used (Sweatt, 2010).

Long-term memory can be divided into declarative memory and nondeclarative memory, also referred to as explicit and implicit memory, respectively (Cohen and Squire, 1980; Eichenbaum et al., 1996; Kolb and Whishaw, 2011; Reber et al., 1998; Schacter, 1992; Squire, 1992; Tulving, 1992). Declarative memories are memories for facts and events, both semantic (factual information and knowledge e.g. definitions of terms) and episodic (autobiographical events and personal experiences e.g. the events that took place on a special day in ones life) (Cohen and Squire, 1980; Squire, 1992; Tulving, 1992). Declarative memories are consciously recalled and are primarily dependent on medial temporal lobe structures for initial encoding (Gaffan, 1974; Hirsh, 1974; Squire, 1982). Nondeclarative memories include procedural memories such as motor memories, skills and habits, emotional memories, conditioning, and priming (Squire, 1992). Nondeclarative memories demonstrate knowledge but do not explicitly recall information. These types of memories depend on different brain structures depending on the demands of the task (Squire, 1992). For example, skill learning and habits depend on the striatum (Heindel et al., 1989, 1988; Packard et al., 1989; Saint-Cyr et al., 1988; Wang et al., 1990), priming depends on the posterior neocortex (Squire, 1992) and conditioning of skeletal musculature depends on the cerebellum (Thompson, 1986).

As implied above, declarative and nondeclarative memories have distinct neuroanatomies upon which they are dependent. Schott et al. (2005) published a study comparing hemodynamic activity of declarative memory and priming (nondeclarative memory). Priming was associated with hemodynamic decreases in areas such as frontal and occipital brain regions, while declarative memory was associated with increases in parietal and temporal brain regions. Hamann and Squire (1997) reported on patient E.P. who suffered from extensive bilateral hippocampal damage. E.P. had unimpaired perceptual memory (nondeclarative memory) but impaired recognition memory (declarative memory). Patient H.M. showed spared implicit memory function (ability to acquire new motor skills and perceptual learning- nondeclarative memory) but severe declarative memory deficits (inability to perform tasks that extended beyond immediate memory capacity) following bilateral removal of the medial temporal lobes (Milner, 1972; Milner et al., 1968).

As time after initial memory encoding progresses, it is suggested memories are transformed from an initially vulnerable state to a more permanent state through consolidation (Frankland and Bontempi, 2005; Müller and Pilzecker, 1900; Ribot, 1882). Consolidation may involve both synaptic and systems level processes to stabilize the memory against decay. In the present thesis, the terms recent and remote are used to define long-term time points of interest following initial memory encoding. Recent long-term memories refer to a time point 8 days following the end of training procedures. Remote long-term memories refer to a time point 37 days following the end of training procedures. These time 
points are later than what is commonly characterized as short-term memory (seconds to minutes) and are based on time points established by others (Bontempi et al., 1999; Frankland et al., 2004; Kolb and Whishaw, 2011; Lopez et al., 2012; Maviel et al., 2004; McGaugh et al., 2000; Restivo et al., 2009; Sweatt, 2010; Teixeira et al., 2006; Vetere et al., 2011; Weible et al., 2012).

\subsection{Where is Memory Stored in the Brain?}

Karl Lashley (Lashley, 1950), famous for his search for the engram, conducted a series of experiments in the early half of the 20th century in an attempt to determine where memory representations (the engram in Lashleys terminology) are stored in the brain. To determine the locus of memory storage, Lashley lesioned different cortical areas in the rat brain and examined the resulting performance on a maze task. Lashley observed that memory function was disrupted in a manner related to the extent of cortical damage, but not to the location of damage. Thus, Lashley concluded that it was not the location of the lesion, but rather the extent of the lesion that mattered, which led him to suggest memory storage was not localized in a particular brain region, but rather distributed across cortical areas (Bruce, 2001; Josselyn, 2010; Lashley, 1950).

Lashleys findings were likely heavily influenced by the fact that complex tasks, such as maze learning for a rat, require the use of multiple brain structures so that damage to a number of different brain regions was necessary to disrupt the memory for the task. Lashleys distributed view of memory storage has been challenged by case studies involving localized damage to medial temporal lobe structures (Bayley et al., 2005, 2006; Rosenbaum et al., 2000; Scoville and Milner, 1957; Squire et al., 2001; Teng and Squire, 1999). Especially influential were the seminal findings of William Scoville and Brenda Milner (Scoville and Milner, 1957), which showed a localized and severe effect of bilateral removal of the medial temporal lobes on memory. Animal research has shown similar localized effects (Clark et al., 2005a,b; McDonald and White, 1993, 1994, 1995; Morris et al., 1982; Packard et al., 1989; Poldrack and Packard, 2003). Results from these experiments suggest that memory function and storage may be localized to certain subcortical brain structures.

Multiple brain systems mediate different types of memory. These multiple memory systems are hypothesised to be able to act independently in the processing of select forms of memory representations (White and McDonald, 2002). For example, declarative memories are associated most strongly with the medial temporal region (particularly the hippocampus) (Gaffan, 1974; Hamann and Squire, 1997; Hirsh, 1974; Milner, 1972; Milner et al., 1968; Scoville and Milner, 1957; Squire, 1982). Motor memories, including habits, motor skills and response learning, have been mapped primarily to areas including the cerebellum and basal ganglia (Lalonde and Botez, 1990; Marr, 1969; Packard, 2009). Emotional memories have been mapped to areas including the amygdala (Gale et al., 2004; Johansen et al., 2011) and hypothalamus (Ledoux, 1993; Trogrlic et al., 2011). 
The quintessential case showing localized memory function was patient H.M. who underwent a bilateral medial temporal lobe resection at the age of 27 (Scoville and Milner, 1957). Following removal of the medial temporal lobes, H.M. demonstrated severe anterograde amnesia in the form of declarative memory deficits as well as temporally graded retrograde amnesia (Corkin, 2002; Milner et al., 1968; Scoville and Milner, 1957). Despite retaining above average intelligence scores, H.M. was not able to recall the names of people he met following his surgery, he could not recall the date or year, and he could not successfully perform simple tasks such as recalling the type of food he had eaten a short time after a meal (Milner et al., 1968). Interestingly, H.M. showed spared nondeclarative memory function (Corkin, 2002; Milner et al., 1968). He was able to improve on tasks that did not require conscious recall of information, such as a mirror-tracing task. These striking memory deficits provided evidence of the pivotal role played by the hippocampus and surrounding structures in declarative memory and spurred the study of memory organization.

In another seminal case study, patient R.B. suffered an ischemic episode that resulted in a bilateral lesion of the CA1 area of the hippocampus (Zola-Morgan et al., 1986). This patient exhibited severe declarative anterograde and retrograde amnesia. Similar to H.M., R.B. displayed deficits on recall tasks (story recall, diagram recall, paired associates), but performed at control levels on tasks that tested implicit memories such as word priming completion tests. The limited and localized brain damage responsible for the memory impairments evidenced in patient R.B. indicated that damage confined to the hippocampus was sufficient and capable of producing profound declarative memory deficits. It is important to note that for both patients H.M and R.B., there was a pattern of impaired anterograde memory loss (the inability to form new memories) and a time-limited retrograde amnesia (the loss of memories from the recent past while memories from the remote past were spared). Many clinical studies have shown a similar link between anterograde and retrograde amnesia for declarative memories and hippocampal damage (Cohen, 1984; Cohen and Squire, 1980; Milner

et al., 1968; Scoville and Milner, 1957; Squire, 1992), leading to investigation of the hippocampus and its role in memory.

\subsection{The Hippocampus}

The hippocampus, located in the medial temporal lobes, is the brain region typically linked to declarative memory. As such, a discussion of its anatomy and function is pertinent.

\subsubsection{Neuroanatomy}

The anatomical regions that comprise the hippocampus can be separated into the hippocampus proper and the hippocampal formation. The hippocampus proper consists of three main regions named the cornu Ammonis (CA)1, CA2, and CA3. The hippocampal formation includes these regions along with others: 
the dentate gyrus (DG), entorhinal cortex (EC), subiculum (SB), parasubiculum and presubiculum (Figure 1.1 displays a coronal section of the rat hippocampus as it represents the species under study in the present thesis).

Primarily, but not exclusively, the hippocampus is connected in a unidirectional circuit, with the bulk of synaptic inputs coming from sensory cortices via the EC or from within the borders of the hippocampus itself. The majority of synaptic inputs to the DG come from the EC, forming part of the Perforant pathway, first described by Cajal (Cajal, 1893). A less prominent input has also been shown to come into the DG via the parasubiculum and presubiculum (Kohler, 1985). Granule cells are the principal cells of the DG that predominantly project to the CA3 region (Blackstad et al., 1970; Claiborne et al., 1986; Gaarskjaer, 1978; Jaffe and Gutierrez, 2007; Swanson et al., 1978), forming the Mossy Fibre pathway (Cajal, 1893). The CA3 also receives a direct cortical projection from the EC (Witter, 1993), some subcortical structures (such as the amygdala) (Pikkarainen et al., 1999; Pitkanen et al., 2000) and is heavily innervated by its own neurons both from the ipsilateral (recurrent collaterals) and contralateral hemispheres (Ishizuka et al., 1990; Li et al., 1994). The CA3 projects to the CA1, referred to as the Schaffer Collateral pathway, forming synaptic contacts on the proximal apical dendrites (Ishizuka et al., 1990; Li et al., 1994; Schaffer, 1892). The CA1 also receives input directly from the EC where synaptic contacts are made on the distal aspects of the apical dendrites (Witter et al., 1988). The CA1 has reciprocal connections to the subiculum (Amaral et al., 1991; Tamamaki et al., 1987) and EC (Naber et al., 2001). Pyramidal cells are the principal cell types of both the CA3 and CA1. The subiculum projects to the para- and pre- subiculum, and shares reciprocal connections with the EC (Caballero-Bleda and Witter, 1993), with both structures containing pyramidal cells as their principal cell type. The subiculum has substantial efferent projections to brain regions outside the hippocampus such as cortical areas, subcortical structures and diencephalic structures (Canteras and Swanson, 1992; Donovan and Wyss, 1983; Groenewegen et al., 1987; Swanson and Cowan, 1975; Swanson et al., 1981; Witter et al., 1990). The EC projects to and receives input from most of the structures within the hippocampal formation and acts as the main point of entrance for sensory information into the hippocampus and the main point of exit for processed information. The connections between the EC and cortical areas are largely reciprocal in nature, with the EC receiving inputs from areas such as the infralimbic, prelimbic and anterior cingulate cortices and projecting back to these same areas (Insausti et al., 1997; Krettek and Price, 1977; Witter, 1997).

\subsubsection{Memory}

The hippocampus critically contributes to the initial encoding and storage of declarative memory representations (Cohen and Eichenbaum, 1993; Scoville and Milner, 1957; Squire, 1992). Damage to the hippocampus and surrounding areas in humans results in memory impairment in the form of declarative anterograde and temporally graded retrograde amnesia, while nondeclarative memory 
is spared (Bayley et al., 2005, 2006; Kapur and Brooks, 1999; Manns et al., 2003; Scoville and Milner, 1957; Zola-Morgan et al., 1986). The hippocampus plays a role in both the encoding and retrieval of declarative memories. Electrophysiological and neuroimaging experiments have revealed greater levels of hippocampal activity during the encoding of more successfully remembered items such as learned words and word-pairs (Cameron et al., 2001; Fell et al., 2003; Fernández et al., 1999, 2002; Paller and Wagner, 2002). Greater hippocampal activity has been observed during more successful retrieval of word-drawing associations and face-name pairs (Eldridge et al., 2000; Gabrieli, 1997; Kirwan and Stark, 2004; Stark and Okado, 2003; Stark and Squire, 2000, 2001). In addition, hippocampal-damaged patients are able to acquire a number of nondeclarative memories such as perceptual motor tasks (Milner, 1962), categorization tasks (Posner and Keele, 1968), and priming tasks (Warrington and Weiskrantz, 1968, 1974), illustrating the importance of the hippocampus in declarative, but not nondeclarative, memories.

\subsubsection{Spatial Function}

Medial temporal lobe damage, particularly hippocampal damage, in animals and humans disrupts spatial memory (Bartsch et al., 2010; Broadbent et al., 2004, 2006; Burgess, 2008; Clark et al., 2005a,b; D'Hooge and De Deyn, 2001; Dupret et al., 2010; Ekstrom et al., 2003; Goodrich-Hunsaker and Hopkins, 2010; Hassabis et al., 2009; Holdstock et al., 2000; Jarrard, 1983; Morris et al., 1982; Olton and Papas, 1979; Parsons and Davis, 2011; Ramos, 2009; Remondes and Schuman, 2004; Sutherland et al., 2001, 1983; Teixeira et al., 2006; Wiltgen et al., 2010). Pivotal to this finding was the discovery of place cells in the hippocampus by O'Keefe and Dostrovsky in 1971. Place cells are specialized cells that code for a particular spatial location and show an increased firing rate when, for example, an animal is in a specific area or place field. O'Keefe and Dostrovsky implanted electrodes in the dorsal hippocampus of freely moving animals to investigate the neural activity of rats in distinct locations. Rats in a certain area of the testing apparatus and oriented in a specific direction showed high rates of activity in a subset of neurons, indicating those neurons exhibited a preference for spatial orientation. Other neurons showed elevated firing rates when the rats were in different locations. Following the discovery of place cells, O'Keefe and Nadel (O'Keefe and Nadel, 1978) proposed the hippocampus was primarily responsible for compiling and forming a spatial cognitive map of the external environment.

Rats and humans may make use of both egocentric and allocentric strategies when processing spatial relationships. Egocentric strategies are frameworks of the environment that move with the self, while allocentric strategies are frameworks of the environment which remain fixed in the environment and do not change as a function of ones own movement (Burgess et al., 2002). The hippocampus is particularly important in processing allocentric information, but not as important in processing egocentric information (Nadel and Hardt, 2004). Evidence from imaging studies has shown allocentric information processing is 
associated with increased hippocampal activity (Bohbot et al., 2004; Parslow et al., 2004; Zaehle et al., 2007). Studies on patients with hippocampal damage reported impaired performance on tasks that required allocentric information (Bohbot et al., 2004; Feigenbaum and Morris, 2004; Holdstock et al., 2000). Forming a cognitive map of the environment, as is accomplished in allocentric processing, requires the integration of landmarks in the environment and depends on hippocampal function (Bohbot et al., 2004; Nadel and Hardt, 2004; O'Keefe and Nadel, 1978).

Spatial memory tasks are an attractive measure in animal research as they provide a simple way of testing hippocampal function through examining performance on a task, such as a maze. Measures of task performance, such as incorrect turns made in a maze, time to complete a maze, or the search strategy used, may be used as an indication of spatial memory and, potentially, hippocampal function. Evidence from rodent (Broadbent et al., 2004, 2006; Clark et al., 2005a,b; D'Hooge and De Deyn, 2001; Morris et al., 1982; Parsons and Davis, 2011; Ramos, 2009; Remondes and Schuman, 2004; Sutherland et al., 2001, 1983; Teixeira et al., 2006; Wiltgen et al., 2010) and human (Burgess, 2008; Dupret et al., 2010; Ekstrom et al., 2003; Hassabis et al., 2009; Holdstock et al., 2000) studies complement one another, suggesting a role for the hippocampus in the acquisition and encoding of spatial information. The significance of the hippocampus in spatial navigation functions has been demonstrated through impaired behavioural performance on the radial arm maze task (Jarrard, 1983; Olton and Papas, 1979) and the hidden platform Morris water maze task (Morris et al., 1986, 1982) following hippocampal damage. Human patients with hippocampal lesions have shown similar deficits in performance on analogous spatial tasks in the form of a virtual radial arm maze (Goodrich-Hunsaker and Hopkins, 2010) and virtual water maze task (Bartsch et al., 2010).

A human study examined hippocampal volume in London taxi drivers. Taxi drivers in London are required to undergo extensive training, lasting about two years, to learn how to properly navigate the city (Maguire et al., 2000). Imaging results revealed experienced London taxi drivers had significantly larger posterior hippocampi compared to control subjects. Analyses revealed that right posterior hippocampal volume was positively correlated to the amount of time spent as a taxi driver. This indicated that posterior hippocampal volume was likely an acquired change, and not a predisposition in taxi drivers, which suggested an ongoing role of the hippocampus in spatial navigation memory. The results indicated the capacity for plastic changes in the hippocampus according to environmental demands and highlighted the importance of the hippocampus in spatial navigation.

Individual case studies in humans with hippocampal damage have advanced knowledge regarding the role of the hippocampus in spatial memory function. Patient E.P. suffered bilateral hippocampal damage. He was unable to successfully navigate through the neighbourhood to which he moved following his hippocampal damage (Teng and Squire, 1999). However, E.P. was able to successfully navigate through the neighbourhood he had resided as a youth, and demonstrated both familiar and novel navigation strategies (Teng and Squire, 
1999). E.P.s findings indicated a role for the hippocampus in acquiring spatial memories, but not necessarily retrieving them (Teng and Squire, 1999). Patient K.C. also suffered from bilateral hippocampal damage. On spatial tests, K.C. was impaired in recalling detailed spatial information regardless of when the spatial information had been acquired, suggesting a permanent role of the hippocampus in location details (Rosenbaum et al., 2000). Patient Y.R. suffered from bilateral hippocampal damage and showed significant deficits in recalling allocentric, but not egocentric, spatial information (Holdstock et al., 2000). Patient H.M., who had removal of the medial temporal lobes, showed severe deficits in spatial memory tasks such as remembering the location of a house to which he moved five years following his operation in 1958 (Milner et al., 1968). Human case studies provide insight into the functional role of the hippocampus. However, factors such as the extent of the hippocampal lesion, the amount of damage to surrounding areas, and tests used to determine impairments can vary between patients making it difficult to determine the precise role of the hippocampus.

Not only is there cross-species conservation of function in the hippocampus between rats and humans, but evidence from avian investigations has also supported a role for the hippocampus in spatial memory. Avian species provide an appropriate model when investigating spatial behaviour because their behaviours often include sophisticated spatial memory functions such as homing, food storing, and other navigation skills (Lee et al., 1998). Homing pigeons are selectively bred based on the ability to find their way home over very long distances. They are hypothesised to use the hippocampus to acquire cognitive spatial maps, which enables them to find their way home even when released in a novel area (Bingman et al., 1990). Hippocampal ablation in homing pigeons has revealed a deficit in the ability to navigate home from distant, unfamiliar locations, suggesting the hippocampus is important in acquiring navigational maps (Bingman et al., 1990). Food storing birds store and retrieve hoarded food in up to thousands of food caches. These birds have larger hippocampal volumes compared to non-food storing birds (differences which cannot be accounted for due to allometric associations between body size and hippocampal size) (Krebs et al., 1989; Sherry et al., 1989). Further analyses revealed that hippocampal volume was related to the amount of food storing behaviour birds engaged in: Birds that relied heavily on food storing had increased hippocampal volume compared to those who relied less on food storing behaviour (Basil et al., 1996; Hampton et al., 1995; Healy and Krebs, 1993). These avian data emphasize the conservation of hippocampal spatial function across species and demonstrate the importance of the hippocampus in forming cognitive spatial maps.

\subsection{The Anterior Cingulate Cortex}

The anterior cingulate cortex (ACC), the frontal part of the cingulate cortex, has typically been associated with higher cognitive functions such as impulse 
control (Aharoni et al., 2013), emotion (Allman et al., 2001; Etkin et al., 2011) attention (Weissman et al., 2005) and error detection (Bush et al., 2000; Cohen et al., 2000; Gehring and Knight, 2000). More recently, it has been linked to remote memory. Research has showed increased activity in the ACC on tests of remote memory (Bontempi et al., 1999; Frankland et al., 2004; Lopez et al., 2012; Maviel et al., 2004; Teixeira et al., 2006; Weible et al., 2012), performance deficits on remote memory tests following ACC inactivation, (Frankland et al., 2004; Lopez et al., 2012; Maviel et al., 2004; Restivo et al., 2009; Teixeira et al., 2006) and structural changes (including increased spine density and growth) indicative of memory storage in the ACC at remote time points (Restivo et al., 2009; Vetere et al., 2011).

\subsubsection{Neuroanatomy}

The ACC is part of the cingulate cortex, a large component of the limbic lobe, which wraps around the corpus collosum (Vogt et al., 2004). In humans, the ACC is defined as Brodmanns area 24 (Brodmann, 1909). The cingulate cortex is an area that receives and integrates inputs from many sources (Bush et al., 2000). It is composed of anterior and posterior regions. Historically, the anterior region has been associated with higher cognitive functions (executive functions) while the posterior region has been associated with evaluative functions, such as monitoring sensory events (Vogt et al., 1992). The ACC may also be separated into rostral and caudal components. Typically, the rostral component of the ACC is associated with affective processes, and the caudal portion associated with cognitive functions (Devinsky et al., 1995).

The rat ACC is a part of the prefrontal cortex, specifically the medial prefrontal cortex (Öngür and Price, 2000). This cortical area is morphologically heterogeneous. Many different cell types are found in this area, including pyramidal cells, multipolar cells, bitufted cells and bipolar cells (Vogt and Peters, 1981). Within the ACC are rostral and caudal regions. The rostral ACC is strongly associated with the infralimbic and prelimbic areas of the prefrontal cortex (Jones et al., 2005; Shibata and Naito, 2008). Animal research investigating remote recall has largely focused on the caudal region of the ACC. The ACC is highly interconnected with other prefrontal regions, as well as sensory, motor and limbic cortices (Jones et al., 2005; Uylings et al., 2003). With exten-

sive connections, the ACC may be well situated to coordinate the retrieval of remote memories from cortical networks.

\subsubsection{Connections between the Hippocampus and Ante- rior Cingulate Cortex}

The ACC receives afferents from many cortical regions. Hoover and Vertes (2007) demonstrated projections from the CA1 of the hippocampus and subiculum to the dorsal medial prefrontal cortex, including the ACC. A report from Cenquizca and Swanson (2007) substantiated this finding, indicating a moderately dense projection from the dorsal CA1 to the rostrocaudal length of 
the dorsal and ventral retrosplenial areas, which spills over into the caudal portion of the ACC. The retrosplenial cortex is considered an important hub for information flow from limbic structures (Van Groen and Wyss, 2003) and shares reciprocal connections with the hippocampus and parahippocampal region (Vann et al., 2009). The retrosplenial cortex also projects strongly to the ACC (Hoover and Vertes, 2007), suggesting another route through which the hippocampus and ACC may interact. In examining the efferent connections from the ACC, it is interesting to note that evidence suggests projections do not target the hippocampus directly. Instead, extensive projections seem to target the parahippocampal region, suggesting information from the ACC is processed in parahippocampal regions prior to entering the hippocampal formation (Jones and Witter, 2007).

Systems consolidation (discussed later) suggests that as time passes there is a shift in the balance of neural activity from the hippocampus to cortical regions in the representation of a remote memory. There are prominent ipsilateral, unidirectional, monosynaptic projections from the hippocampus to the prefrontal cortex in rats (Ferino et al., 1987; Jay et al., 1989; Jay and Witter, 1991; Swanson et al., 1981). The hippocampal-prefrontal cortex pathway is of functional importance for information transfer. Stimulation of the CA1 can induce LTP in the prefrontal cortex (Jay et al., 1996; Jay and Witter, 1991) indicating the capacity for synaptic plasticity in this circuit. A study examining synaptic efficacy during learning revealed different dynamics between hippocampal inputs from the entorhinal cortex and hippocampal outputs to the prefrontal cortex (Doyere et al., 1993). Briefly, synaptic potentiation at hippocampal inputs occurred early during learning while synaptic potentiation at prefrontal hippocampal output synapses occurred at a delayed time point following maximal learning. These electrophysiological results suggest the hippocampal-prefrontal cortex pathway is involved in late consolidation and the stabilization of a cortical representation (Laroche and Davis, 2000).

The Papez circuit is a network showcasing connections between the hippocampal formation and cortical areas, as well as connections between a number of other limbic structures. The Papez circuit was originally proposed to play a role in emotion (Papez, 1937), however, a role in memory storage has also been suggested (Aggleton and Brown, 1999; Barbizet, 1963; Carlesimo, 2012; Thomas et al., 2011; Valenstein et al., 1987). As described by Papez (1937), the hippocampus receives input from the EC and passes it to the SB. The SB connects to the mammillary bodies of the hypothalamus via the fornix (a major fibre system associated with outgoing signals from the hippocampus). From the mammillary bodies, projections travel through the mammillothalamic tract to the anterior thalamic nucleus. From the anterior thalamic nuclei, the circuit connects to the cingulate cortex, and the cingulate cortex projects back to the EC through the cingulum. This circuit highlights connections between the hippocampus and the cingulate cortex, indirectly through the mammillothalamic tract to the anterior thalamic nucleus, or directly via the EC. This circuit may represent the neural substrate by which information is passed during systems consolidation. 
Research has indicated that successful spatial task performance is associated with stable place fields (Kentros et al., 2004). Altered responsivity in the hippocampus, resulting in less stable firing fields in place cells, occurred following prefrontal cortex lesions (Kyd and Bilkey, 2003). It was suggested a loss of attentional control resulted in less stable environmental cues exerting a stronger influence than they would normally (Kyd and Bilkey, 2003). These results posit that attention, a function attributed to the ACC, can modulate the stability of hippocampal place fields and thus memory (Rowland and Kentros, 2008), suggesting an important relationship between these structures relating to spatial memory storage. Furthermore, disrupting the connections between the hippocampus and the prefrontal cortex result in deficits on a number of spatial behavioural tasks such as the water maze (Wang and Cai, 2008), T-maze (Wang et al., 2006), and the Hebb-Williams maze (Churchwell et al., 2010), highlighting the significant relationship between these structures, especially in spatial memory processes.

\subsection{Behavioural Tasks}

\subsubsection{Water Maze}

The water maze (WM) is a classic test of spatial learning and memory in rodents. Developed by Richard Morris in 1981 (Morris, 1981), rats are placed in a pool of water with a hidden platform located just below the surface of the water, affording escape from the water (Morris, 1981). The design of the water maze differentiates between proximal (detectable cues of an object of interest) and distal (cues relative to an invisible object of interest) cues, and tests the use of allocentric spatial reference memory (Morris, 1981). Distal cues are located around the testing room to allow rats to learn the location of the submerged platform using allocentric information. A single WM trial typically lasts about 60 seconds. If the rat does not find the hidden platform within this time, the experimenter will lead the rat to the platform. The rat will remain on the platform for 15-30 seconds before being removed by the experimenter. During this time, information relating to the location of the hidden platform relative to extra-maze cues may be gathered. If the hidden platform remains in the same position for subsequent trials, rats easily learn the location of the platform. Spatial learning is commonly measured by the latency to reach the hidden platform. As rats learn the location of the hidden platform, the time it takes for them to reach the platform decreases.

The water maze task is often accompanied by a probe trial(s) following training (Figure 1.2). During a probe trial, rats are released into the pool as during training, however, the platform is removed from the pool. The purpose of a probe trial is to analyze the search strategy for the platform location. Water maze software is able to track performance during a probe trial and provide in-

formation on a number of variables of interest. The percent time spent in each of the four quadrants of the pool is often analyzed. The quadrants include 1) 
the target quadrant, the quadrant of the pool where the platform was located during training, 2) the opposite quadrant, the quadrant of the pool furthest removed from the target quadrant, and 3) two adjacent quadrants. It is expected that rats spend $25 \%$ of their search time in any given quadrant due to chance. A strong memory for the platform location is indicated by rats spending above chance amounts of time searching in the quadrant where the platform was located during training i.e. spending significantly more than $25 \%$ of their search time in the target quadrant.

The percent of time spent in the target annulus area is often analyzed as well. The target annulus represents an area closely associated with the precise location of the escape platform. This measure is similar to measuring the time spent in the target quadrant; however, it is a more accurate measure as it more precisely represents the location of the platform and neglects time spent along the wall, which would not be indicative of an informed search path. The number of passes made through the location where the platform was located during training can be measured. A greater number of passes through the platform location indicates a strong memory for the platform location. Thigmotaxis, swimming around the perimeter of the pool, is another factor frequently measured. Thigmotaxis is an ineffective search strategy but is common at the beginning of training on the WM task. Once rats have learned the location of the hidden platform, thigmotactic behaviour generally decreases. Additional information such as the amount of time spent floating, swim speed, and heading direction may be analyzed as well. These factors can be analyzed during different time bins during the probe trial. This provides the opportunity to examine initial search patterns separately from later search patterns. It may be the case that initial search patterns focus on the correct area of the pool, while later search patterns include other areas of the pool as continued searching was met with failure in finding the platform (which was removed from the pool during the probe trial). The analysis of these factors provide insight into the search patterns of rats during the probe test, allowing one to examine the area of the pool rats spent most of their time searching, offering an indication of the strength of the memory for the platform location.

The water maze is a spatial navigation task that is dependent on the hippocampus (Morris et al., 1982). Bilateral lesions or inactivations of the hippocampus and associated areas (dentate gyrus, subiculum, entorhinal cortex) impair the performance of animals on this task (Broadbent et al., 2006; Clark et al., 2005a,b; D'Hooge and De Deyn, 2001; Morris et al., 1982; Ramos, 2009; Remondes and Schuman, 2004; Sutherland et al., 2001, 1983; Teixeira et al., 2006; Wiltgen et al., 2010). Morris et al. (1982) reported the first findings of hippocampal-lesioned rats and their performance on the water maze. In this experiment, there was a place-navigation and a cue-navigation task where the platform was hidden or visible, respectively. Animals with hippocampal lesions and trained on the place-navigation task (hidden platform) showed significant impairments in locating the hidden platform. These impairments were not attributed to locomotor or swimming deficits. Rats with hippocampal lesions took longer to find the platform than control rats, did not leave the start lo- 
cation heading toward the hidden platform, and swam a more circuitous path (exhibiting more thigmotaxis) than control animals. Hippocampal-lesioned animals performed similar to control animals in the cue-navigation task. Many studies have since reported similar hidden-platform water maze deficits, but intact cue-navigation performance, following hippocampal lesions (Benhamou and Poucet, 1995; Brandeis et al., 1989; McNaughton et al., 1996; Sutherland et al., 1983; Whishaw et al., 1997). The degree of impairment on the water maze task in hippocampal-lesioned animals is correlated with the degree of damage in the dorsal hippocampus (Moser et al., 1993, 1995).

\subsubsection{Radial Arm Maze}

The Radial Arm Maze (RAM) is a spatial task in which a number of arms, baited with a food reward at their end, radiate out from a central location (Olton and Samuelson, 1976) (Figure 1.3). The successful completion of the maze requires the rat to visit all arms to obtain the food reward, without reentering an arm that has already been visited (i.e., the rat cannot retrace its steps). The RAM requires the rat to return to the central arena prior to making another choice, thus requiring the rat to decide between all of the maze arms. A variation on the RAM task involves baiting only a subset of arms (Bouffard and Jarrard, 1988; Olton and Papas, 1979). In this task, the baited arms are always baited and the non-baited arms are never baited. Rats receive repeated trials with continuously baited arms within and across days. Extramaze (distal cues) are available. Rats learn which arms are baited and travel only down these arms without re-entering them. An error is defined as re-entering an already entered arm where the food reward has been taken, or entering an unbaited arm. This spatial task is hippocampal-dependent and requires reference and working memory (Bouffard and Jarrard, 1988; Jarrard, 1978; Olton and Papas, 1979). The reference memory portion of the task includes remembering which arms are baited and which arms are not. The working memory portion of the task involves remembering which baited arm an animal has already collected the reward from, so as to not re-enter that arm. Animals with a normal functioning hippocampus are very successful in completing the RAM task, rarely re-entering arms. Errors on the RAM task include entering arms that are never baited (reference memory errors) and re-entering arms (working memory errors).

Lesion studies demonstrate the importance of a functioning hippocampus in the RAM task (Bouffard and Jarrard, 1988; Jarrard, 1976, 1978; Olton and Papas, 1979; Olton et al., 1978; Winocur, 1982). Winocur (1982) lesioned the hippocampus of rats and found impaired performance on a standard radial arm maze task where rats had to use extramaze cues for successful performance. Hippocampal-lesioned rats showed a greater number of incorrect arm choices compared to controls. Performance of hippocampal-lesioned rats improved when an intramaze-cued version of the radial arm maze was used. Bouffard and Jarrard (1988) employed a RAM task where only a subset of the arms was baited with a food reward. Hippocampal-lesioned rats in this experiment demonstrated a number of reference memory and working memory errors, indicating the im- 
portance of the hippocampus in successful performance this task. Amnesic patients with hippocampal damage performed a virtual RAM task with 4 of 8 arms baited (Goodrich-Hunsaker and Hopkins, 2010). Amnesic participants made a greater number of errors compared to control participants evidenced by entering non-baited arms and re-entering arms already visited. The deficits found in these human patients on the RAM are strikingly similar to the deficits noted in animal research, and suggest the hippocampus is an important structure involved in successful performance on the RAM task. In addition to lesion studies, activation studies have shown increased c-Fos activity in the hippocampus during radial arm maze training and testing (He et al., 2002; Vann et al., 2000), supporting the finding that the hippocampus is a critical structure involved in the successful completion of this task.

\subsubsection{Operant Conditioning}

Non-spatial tasks are minimally affected or altogether unaffected by damage to the hippocampus. One such task is operant conditioning (OP). Operant conditioning can be defined as behaviour modification that utilizes positive or negative reinforcement and positive or negative punishment to alter behaviour (Skinner, 1937). The operant conditioning discussed here focuses on positive reinforcement by lever-pressing for food, and is not hippocampal-dependent (Carvalho et al., 2001; Corbit and Balleine, 2000; Gallagher and Hollandt, 1992; Shull and Holloway, 1985). The procedure used placed animals in an operant conditioning box where there were two levers: an active lever and an inactive lever (Figure 1.4). Animals were required to press the active lever two times in a row to receive a food reward. Upon doing this, the chamber light extinguished. Presses on the inactive lever elicited no food reward. Animals learned over time that a reward was given after pressing on the active lever 2 times in a row (Holahan et al., 2010, 2012).

Gallagher and Hollandt (1992) used a within subjects study design to examine the effect of hippocampal lesions on a spatial learning task compared to a non-spatial operant discrimination task. Hippocampal-lesioned animals showed severe deficits on the spatial task, but performed similarly to controls on the operant discrimination task. Corbit and Balleine (2000) investigated the effects of dorsal hippocampal lesions on instrumental conditioning. Hippocampal-lesioned animals did not exhibit any deficits on the acquisition of an instrumental operant task. Carvalho et al. (2001) tested $\alpha$-CaMKIIT286A mice, that is mice that exhibit deficits in hippocampal LTP and spatial learning. These mice showed normal acquisition on an instrumental conditioning task, displaying no deficit in operant conditioning performance.

\subsection{Molecular Mechanisms of Memory}

One of the obstacles in memory research is uncovering how, amidst constant metabolic turnover in the brain, memories are stored and able to become stable, 
durable and persist through time. Short-term memories are able to last minutes among the chaos of activity between and within discrete neural networks. Even more impressive, long-term memories are able to last a lifetime. This begs the question: how are memories able to persist?

Synaptic plasticity, a change in the strength of connections between neurons due to experience, is believed to represent a process through which learning and memories are stored (Martin et al., 2000). A synapse is malleable and able to respond to experience. Learning can bring about cellular and molecular changes that can influence the strength of connections between neurons. If these changes persist, memories are believed to be represented (Benfenati, 2007; Ho et al., 2011; Martin et al., 2000). Long-term potentiation (LTP) represents one of the best-characterized illustrations of synaptic plasticity and is one of the prime candidates for the storage of memories in the brain.

\subsubsection{Long-Term Potentiation}

Long-term potentiation (LTP), a long-lasting change in synaptic strength resulting from stimulation, is believed to be a key cellular process underlying learning and memory (Bliss and Lø mo, 1973). LTP is induced rapidly by high frequency stimulation and is characterized by the strengthening of a synapse between simultaneously activated neurons. LTP is long lasting, and once induced, may persist for hours, days or longer if the stimulation is repeated.

Findings described by Terje Lømo (Lø mo, 1966) regarding a potentiated response in the hippocampus following the cessation of stimulation, drove Lømo and Tim Bliss to their seminal study of LTP in 1973. Bliss and Lømo used electrodes to stimulate the perforant pathway of the hippocampus, and recorded evoked responses in the dentate gyrus of an anaesthetized rabbit. They found that repetitive stimulation led to a potentiated response in granule cells of the dentate, lasting up to $10 \mathrm{hrs}$. These findings suggested a potential cellular process for long-term information storage, as events that occurred hours prior exerted long-lasting effects on the synapse and cell responses. Subsequent research has supported Bliss and Lømos findings (Andersen et al., 1980; Bindman et al., 1979; Bliss and Gardner-Medwin, 1973; Bliss et al., 1983; Dunwiddie and Lynch, 1978; Haas and Rose, 1982; McNaughton, 1982). Based on its persistence and longevity, LTP is believed to be a key cellular process underlying learning and memory.

LTP can be dissociated into different phases: LTP1 (or early LTP), LTP2 and LTP3 (or Late LTP). LTP1 is defined by weak stimulus induction and a rapid onset and decay (Raymond, 2007). LTP1 lasts approximately 1-3 hours and is independent of protein synthesis; instead, this phase relies upon the alteration of pre-existing proteins by phosphorylation (Benfenati, 2007; Lynch et al., 2004; Raymond, 2007; Sweatt, 1999). The existence of an intermediary phase, LTP2, is disputed. However, research supports the idea of a transitional phase between LTP1 and LTP3 processes, which shifts from transcription independent to transcription dependent processes (Huang and Kandel, 2005; Kelleher et al., 2004; Otani et al., 1989). LTP2 is defined by a dependence on protein synthesis 
in the absence of recent gene transcription (Raymond, 2007). Translation occurs on pre-existing mRNA in the absence of recent gene transcription (Raymond, 2007). LTP3 is both protein synthesis and gene transcription dependent (Benfenati, 2007; Lynch et al., 2004; Raymond, 2007; Sweatt, 1999). It is induced by repeated stimulation and is the most persistent form of LTP (Abraham and Williams, 2003; Benfenati, 2007; Lynch et al., 2004; Raymond, 2007; Sweatt, 1999). LTP3 can last from hours to days to weeks (Lynch et al., 2004; Raymond, 2007). These different phases of LTP are often used as experimental models of memory. LTP1 is frequently used as a model of short-term memory, while LTP3 is frequently used as a model of long-term memory.

The reliability of LTP has prompted its use as an experimental cellular model to determine the molecular mechanisms underlying memory. Short-term memories are fragile, unstable and liable to disruptions. It is believed the molecular basis of short-term memory involves transient changes in pre-existing neuronal networks, accomplished through alterations in intracellular pathways (Benfenati, 2007; Dudai, 2004; Kandel, 2001; Laroche and Davis, 2000; Sweatt, 1999; Wang et al., 2006). Protein synthesis is not critical to short-term memory formation, as protein synthesis inhibition does not interfere with this process (as seen in LTP1) (Bliss and Collingridge, 1993; Frey et al., 1988; Kandel, 2012; Krug et al., 1984; Matthies et al., 1990; Squire and Barondes, 1972). Longterm memory depends on protein synthesis and gene transcription (Benfenati, 2007; Dudai, 2004; Kandel, 2001, 2012; Laroche and Davis, 2000; Roberson and Sweatt, 1999; Squire and Barondes, 1972; Sweatt, 1999). The longevity of long-term memories implies that the molecular representations must persist throughout time to ensure their stability.

\section{Induction}

LTP is induced through electrical stimulation in presynaptic axons, which leads to the release of glutamate capable of activating postsynaptic NMDARs (Benfenati, 2007; Lynch et al., 2004; Raymond, 2007; Sweatt, 1999). In this type of LTP, the NMDAR is a fundamental and crucial component of LTP induction. NMDAR blockade interferes with both LTP induction and memory storage, lending support to the idea of LTP as an underlying process for memory storage (Bashir et al., 1990; Coan et al., 1987; Collingridge et al., 1983; Morris, 1989; Morris et al., 1986). The NMDAR is a voltage-dependent, glutamategated receptor with a $\mathrm{Mg}^{++}$blocked pore, which, unless removed, prevents the flow of calcium ions through the receptor (Ascher and Nowak, 1988; Bliss and Collingridge, 1993; Monaghan and Jane, 2009; Sweatt, 1999). The $\mathrm{Mg}^{++}$block is expelled from the pore by membrane depolarization. Depolarization coupled with the binding of glutamate opens the channel and allows calcium into the post-synaptic cell. Calcium influx results in the activation of second messenger cascades (Benfenati, 2007; Lynch et al., 1983, 2004; Raymond, 2007; Sweatt, 1999), and sets in motion processes that underlie the induction of LTP. 


\section{Expression}

The exact pathways necessary for inducing LTP are disputed. It seems likely that many signalling cascades are capable of triggering LTP indicating several potentially important kinases. A second messenger activated by calcium influx and a key player in E-LTP, is calcium /calmodulin-dependent protein kinase II (CaMKII) (Bliss and Collingridge, 1993; Ito et al., 1991; Lynch et al., 1983; Malenka et al., 1989; Malinow et al., 1989; Mody et al., 1984; Reymann et al., 1988). CaMKII represents one of the best-studied second messengers in ELTP and short-term memory. Calcium entering the postsynaptic cell stimulates CaMKII activation, resulting in its translocation to the postsynaptic zone of the NMDAR. Once activated, CaMKII is able to autophosphorylate itself and sustain its activity independent from calcium binding (Malenka et al., 1989; Malinow et al., 1988; Miller and Kennedy, 1986). In this way, a transient event (the influx of calcium) can result in an event that persists beyond this initial activation (autophosporylation of CaMKII).

CaMKII activation can enhance $\alpha$-amino-3-hydroxy-5-methyl-4-isoxazolepropionic acid receptor (AMPAR) function and facilitate the insertion of AMPARs into the postsynaptic membrane, facilitating LTP expression and resulting in structural rearrangement (Isaac et al., 1996; Kessels and Malinow, 2009; Malenka and Bear, 2004; Malinow et al., 2000; Malinow and Malenka, 2002). In addition, phosphorylation of AMPARs can modify the functional properties of these receptors, an important mechanism in LTP expression (Malenka and Bear, 2004).

\section{Maintenance}

The characteristics surrounding the early phases of LTP, described above, are well defined. What remains unclear, are the mechanisms involved in the maintenance of LTP for days and weeks. It is widely accepted that the late phase of LTP involves protein synthesis and gene transcription (Benfenati, 2007; Dudai, 2004; Kandel, 2001; Laroche and Davis, 2000; Roberson and Sweatt, 1999; Sweatt, 1999). Long-term maintenance may occur through structural rearrangements at synapses where LTP has occurred. Structural rearrangements may include new dendritic spines, enlargement of existing spines, changes in the types of spines present, or increases in dendritic complexity (Bailey and Eric, 1993; Malenka and Bear, 2004; Matsuzaki et al., 2004).

\subsection{Proteins of Interest in Memory Formation and Storage}

\subsection{1 c-Fos}

A nuclear protein and immediate early gene of interest in memory formation, consolidation and retrieval is c-Fos (Dragunow et al., 1989; Kaczmarek and Kamiska, 1989; Katche et al., 2010; Morgan and Curran, 1989; Tischmeyer and 
Grimm, 1999). c-Fos is a regulatory transcription factor associated with neuronal activity and expressed following many forms of synaptic activation (Greenberg and Ziff, 1984; Kovács, 2008; Morgan and Curran, 1989; Tischmeyer and Grimm, 1999). The transcription factor, AP-1, is formed through the dimerization of c-Fos and c-jun members, providing the mechanism through which c-Fos plays a role in gene transcription (Greenberg and Ziff, 1984; Katche et al., 2010; Kovács, 2008). There is evidence to suggest that c-Fos is involved in memory formation and consolidation (Guzowski et al., 2001)(For reviews see (Herdegen and Leah, 1998; Tischmeyer and Grimm, 1999)). Behavioural training, on a number of different types of tasks, has been shown to increase c-Fos protein in the hippocampus (Cammarota et al., 2000; Guzowski et al., 2001; Papa et al., 1995; Vann et al., 2000; Wan et al., 1999; Zhu et al., 1997). c-Fos knockout mice displayed deficits in performance on both the cued and non-cued water maze task (Paylor et al., 1994). Grimm et al. (1997) used a shock-motivated brightness discrimination task to show that a pretraining injection of c-Fos antisense oligodeoxynucleotides (ODN) did not affect task acquisition, but did affect 24hour retention. Animals injected with c-Fos antisense ODNs showed a significantly reduced retention index (calculated from the number of training errors and the number of errors made at the 24 hour retention test), indicating a significant impairment in memory retention compared to control groups. Katche et al. (2010) reported evidence of a delayed wave of c-Fos expression in the hippocampus 24 hours following a strong training protocol. This delayed expression of c-Fos was found to be important for memory persistence and indicates the importance of c-Fos in the consolidation process.

\subsubsection{Activity-regulated cytoskeleton-associated protein}

Activity-regulated cytoskeleton-associated protein/ activity-regulated gene 3.1 (Arc/Arg 3.1) is an immediate early gene (IEG) whose mRNA levels at baseline are low, but become upregulated in the presence of stimulation that results in LTP2/3 (Guzowski et al., 2000; Link et al., 1995; Lyford et al., 1995). Arc mRNA is localized selectively to dendrites that have recently been activated thereby labelling potentiated synapses (Kovács, 2008; Kuhl and Skehel, 1998; Link et al., 1995; Lyford et al., 1995; Steward et al., 1998; Steward and Worley, 2001). Arc protein labeling is associated with memory consolidation, but not acquisition (Guzowski et al., 2000). Guzowski and colleagues infused Arc antisense ODN into the hippocampus prior to spatial water maze training. Controls and Arc antisense animals performed similarly during acquisition, but Arc antisense animals exhibited a deficit in retention when tested 48 hours later. Plath et al. (2006) provided evidence showing Arc knockout mice exhibited deficiencies in LTP2/3 and the formation of long-lasting memories, while LTP1 and short-term memories were not affected. Behavioural tasks used by Plath et al. (2006) included hippocampal-dependent and independent tasks. Impaired spatial learning strategies were displayed by Arc knockout animals when tested on the water maze task. Although these knockout animals showed a preference for the target quadrant, they spent significantly less time in the target quadrant 
compared to wildtype controls and had significantly fewer annulus crossings over the trained platform location. In addition, Arc knockout animals were slower to learn a new platform location. These deficits were not assumed to be due to impairment in sensory-motor functions as swim velocities, time floating and performance on a visible platform task were not significantly different between Arc knockout animals and wildtype controls. Arc knockout animals showed performance deficits in a long-term fear-related memory task. Both wildtypes and knockouts showed a short-lasting fear association to context and tone during acquisition. Retention of this memory was tested 24 hours later. Knockout animals showed significantly lower levels of freezing to both context and tone compared to wildtype controls. A novel object-recognition task was used to determine object-recognition memory. Both wildtypes and knockouts displayed short-term object-recognition memory, evidenced by spending a greater amount of time examining a novel object. Long-term retention tested 24 hours later showed a lack of object-recognition memory in Arc knockout animals evidenced by only barely above chance levels of exploration of a novel object. Arc knockout animals were tested on conditioned taste aversion, a very robust, rapidly-learned long-lasting association memory. Arc knockout animals did not form a long-term conditioned taste aversion memory, despite normal gustatory processing.

Evidence exists to support the idea that Arc plays a role in LTP maintenance (Guzowski et al., 2000; Messaoudi et al., 2007). Guzowski and colleagues (Guzowski et al., 2000) used intrahippocampal infusions of Arc antisense ODNs to inhibit Arc protein expression and determine the effect on LTP. Results indicated that initial LTP amplitude did not significantly differ between animals treated with antisense ODNs and scrambled ODNs, suggesting LTP induction was not affected by the inhibition of Arc protein expression. The rate of decay of LTP in Arc antisense treated animals was much more rapid than that seen in Arc scrambled animals, suggesting Arc protein expression plays a role in the maintenance of LTP. Similar results were found by Messaoudi and colleagues (Messaoudi et al., 2007) who injected Arc antisense two hours after the onset of high frequency stimulation (HFS). Disruption of Arc protein expression at this time caused a rapid reversal of LTP with field excitatory postsynaptic potential recordings returning to baseline levels within 60 minutes. Interestingly, no effect was seen when Arc antisense was injected four hours following HFS, indicating a time sensitive role of Arc in LTP maintenance. Bramham and colleagues (Bramham et al., 2010) have suggested Arc may act by regulating actin activity to promote LTP consolidation, proposing Arc may play a part in stabilizing structural changes at potentiated synapses as part of its role as a cytoskeleton-associated protein.

\subsubsection{Protein Kinase $\mathrm{M} \zeta$}

An attractive candidate for the maintenance of LTM and LTP2/3 is an unusual kinase, protein kinase $\mathrm{M} \zeta(\mathrm{PKM} \zeta)$. PKM $\zeta$ is a constitutively active PKC isoform that lacks the regulatory domain of PKC and contains only the catalytic domain (Hernandez et al., 2003). As a second-messenger independent kinase, 
its activity seems to persist longer than usual for a typical kinase. $\operatorname{PKM} \zeta$ has been shown to play a critical role in maintaining L-LTP (Pastalkova et al., 2006; Serrano et al., 2005). Evidence shows that blocking the activity of PKM $\zeta$ selectively reversed LTP2/3, but had no effect on LTP1 (Pastalkova et al., 2006; Serrano et al., 2005). As a result, it was hypothesized that PKMZ $\zeta$ may be involved in forming and preserving structural changes at synapses thought to be critical in maintaining long-term memories (Serrano et al., 2005; Shao et al., 2011; Shema et al., 2007). PKM $\zeta$ plays an imperative role in LTP, and thus potentially in long-term memory maintenance, such that inhibition hours or days after LTP induction causes LTP2/3 maintenance to be reversed. Locally inhibiting $\mathrm{PKM} \zeta$ days, weeks or months after training can erase long-term memories, both declarative and procedural, illustrating its profound and potent effect on memory storage (Madroñal et al., 2010; Migues et al., 2010; Shema et al., 2007; von Kraus et al., 2010). PKM $\zeta$ s suspected mode of action is through the maintenance of glutamate receptor 2 (GluR2) at the postsynaptic density. Evidence suggests $\operatorname{PKM} \zeta$ blocks a pathway important in removing certain postsynaptic AMPAR, thereby increasing levels of postsynaptic AMPAR persistently (Hernandez et al., 2003; Ling et al., 2006, 2002; Migues et al., 2010; Sacktor, 2008; Shao et al., 2011; Yao et al., 2008).

In neurons, $\mathrm{PKM} \zeta$ mRNA is produced from an internal promoter within the $\mathrm{PKC} \zeta$ gene, which produces a $\zeta$ catalytic domain lacking a regulatory domain. $\mathrm{PKM} \zeta \mathrm{mRNA}$ is shuttled to the dendrites of neurons but is translationally blocked until signalling cascades set off by LTP induction (such as CaMKII, PKA, and NMDAR activation) release the block. Following translation, PKM $\zeta$ binds to and is phosphorylated by phosphoinositide-dependent protein kinase 1 (PDK1), which increases $\mathrm{PKM} \zeta$ s constitutive activity, and maintains increased translation of itself. PKM $\zeta$ regulates AMPAR trafficking by effecting the interaction between N-ethylmaleimide-sensitive factor (NSF) and GluR2, a relationship which, under basal conditions keeps a homeostatic balance of AMPARs to potentiate synaptic transmission by increasing the number of GluR2-containing subunits at postsynaptic sites (Migues et al., 2010; Sacktor, 2008, 2011; Yao et al., 2008).

Although initially very promising, presently the role of PKM $\zeta$ in LTP and memory maintenance is under question. Recently, reports have emerged that call into question the role played by this kinase. Volk and colleagues (Volk et al., 2013) developed conventional and conditional PKC $\zeta / \mathrm{PKM} \zeta$ knockout mice. These mice showed normal hippocampal synaptic transmission and normal performance on learning and memory tasks. Of particular interest in this study, was the effect of zeta inhibitory peptide (ZIP), a peptide frequently used in $\mathrm{PKM} \zeta$ research and believed to be a selective $\mathrm{PKM} \zeta$ pseudosubstrate inhibitor (Shema et al., 2007). ZIP resulted in LTP reversal in knockout mice. The results from Volk et al. (2013) suggest ZIP has either non-specific effects, or, its action is not dependent on PKM $\zeta$. Lee et al. (2013) also used PKC $\zeta / \mathrm{PKM} \zeta$ knockout mice. Knockout mice performed similarly to controls on tests of learning and memory. Of importance, injections of ZIP into the nucleus accumbens of knockout mice impaired reward memory. The results from Lee et al. (2013) 
support the findings of Volk et al. (2013), and suggest ZIP can impair memory through $\operatorname{PKM} \zeta$ independent mechanisms. These findings are particularly troubling as a great deal of work suggesting the importance of PKM $\zeta$ in memory maintenance has been accomplished through the use of ZIP (Migues et al., 2010; Pastalkova et al., 2006; Serrano et al., 2008; Shao et al., 2011; Shema et al., 2007; Yao et al., 2008). Research suggesting ZIP can impair memory through $\mathrm{PKM} \zeta$ independent mechanisms question the importance of this kinase in memory maintenance.

\subsubsection{Growth and Plasticity Associated Protein (GAP-43)}

Growth and plasticity associated protein (GAP-43) is a presynaptic protein involved in axon growth, and is used as a marker of newly formed synapses (Benowitz, Larry and Routtenberg, 1997). GAP-43 is a protein kinase C substrate shown to be important during development (Benowitz, Larry and Routtenberg, 1997; Maier et al., 1999; Meiri et al., 1998; Routtenberg et al., 2000). GAP-43 is highly concentrated in growth cones (Nelson et al., 1989), is associated with regenerative growth (Skene, 1989) and is essential in development, as mice lacking GAP-43 die early in the postnatal period (Strittmatter et al., 1995).

Expression of GAP-43 plays a role in learning and memory processes. Evidence suggests a strong link between the phosphorylation state of GAP-43 and an enhancement of LTP (Gianotti et al., 1992; Lovinger et al., 1985; Lovinger and Routtenberg, 1988). Pascale et al. (2004) demonstrated that GAP-43 protein and mRNA expression levels in the hippocampus were elevated in rats following training on a water maze task compared to swim control rats. Similarly, Young et al. $(2002,2000)$ found increased GAP-43 levels and phosphorylation in the hippocampus following contextual fear conditioning training, control animals did not show this change. Maviel et al. (2004) trained animals on a five arm spatial discrimination task and showed animals tested 30 days following training had increased GAP-43 expression in the ACC, compared to animals tested 1 day following training, showing region-specific changes. Vetere et al. (2012) replicated findings showing an increase in GAP-43 in the ACC at remote time points following contextual fear conditioning training. Additionally, they examined GAP-43 expression following extinction. Interestingly, GAP-43 levels were found to still be high in the ACC following extinction. This sustained increase occurred with a decrease in c-Fos levels and spine loss. This led to the suggestion that high levels of GAP-43 may represent a neurons ability to undergo activity-dependent structural changes.

\subsection{Structural Changes Associated with Long- Term Memory}

The human brain, with an estimated 86 billion neurons (Azevedo et al., 2009), is able to store and recall memories across the lifetime. Structural changes are 
proposed to underlie the storage and persistence of long-term memories (Bailey and Eric, 1993; Trommald et al., 1996; Yang et al., 2009). It has been demonstrated that stimulation, producing LTP, is sufficient to cause persistent structural changes.

Lynch and colleagues provided important research indicating information storage led to structural changes (Lee et al., 1981, 1980). These early studies demonstrated that stimulation producing LTP resulted in an increase in synapse number. This finding has been replicated many times since its initial discovery (Chang and Greenough, 1984; Desmond and Levy, 1983, 1986, 1988; Geinisman et al., 1991). More recently, it has been proposed that circuitry and connectivity changes are important for information storage, not simply new synapse production. Thus, synapse elimination has also been found to be an important mechanism in information storage (Bednarek and Caroni, 2011; De Roo et al., 2008; Holtmaat et al., 2006; Lai et al., 2012; Xu et al., 2009; Yang et al., 2009).

In addition to synapse rearrangement, other changes affecting structural plasticity may represent a basis for memory storage. Dendrites and dendritic spines represent the postsynaptic point of contact in a synapse. Increases in dendritic density have been associated with memory formation (Knafo et al., 2004; Leuner et al., 2003; Restivo et al., 2006, 2009). Structural changes in dendrites may underlie mechanisms associated with long-term memory storage (Bailey and Eric, 1993; Trommald et al., 1996). Increases in dendritic characteristics such as dendritic length, branching and number of spines (collectively called dendritic complexity), serve to increase the available surface area for synaptic contact, increasing the capacity for neuronal connectivity (Cajal, 1909). Greater dendritic length and greater dendritic branching increase the number of possible synaptic contacts and information that may be received by a dendrite (Jan and Jan, 2001). Alterations in dendritic characteristics may reflect increased or decreased significance to incoming synaptic information.

Spine remodelling, which includes both spine formation and elimination, has been suggested to play an important role in structural plasticity (Yang et al., 2009). Yang et al. (2009) demonstrate that only a small proportion of new spines that result from novel experiences persist and survive experiencedependent elimination. Those that do survive are believed to represent a structural basis for memory storage throughout the lifetime. Thus, number of spines or spine density may not always be an effective indicator of memory storage or retention on its own; Spine formation may represent a transient event following experience. Likely, the type of spine (thin, mushroom, etc) is of greater importance for memory persistence. Matsuzaki et al. (2001, 2004) used twophoton uncaging of glutamate to stimulate individual spines. These researchers found evidence suggesting that enlargement of small spines due to stimulation was associated with increased AMPAR expression and that large spines may represent structural traces underlying the storage of long-term memories (Matsuzaki et al., 2001). In support of these findings, low frequency stimulation causing long term depression (LTD- a lasting decrease in synaptic effectiveness (Bear and Abraham, 1996)), has been reported to cause spine shrinkage or disappearance (Okamoto et al., 2004; Zhou et al., 2004). The results from 
these studies suggested a difference in form and function between small and large spines. It was suggested small spines are learning spines: those that may change their form rapidly, contain fewer functional AMPA receptors, and are preferred sites for LTP induction (Bourne and Harris, 2007, 2008; Kasai et al., 2003; Matsuzaki, 2007; Matsuzaki et al., 2001, 2004; Yang et al., 2009). Large spines were suggested to be memory spines: those that are more stable, contain greater numbers of AMPA receptors, and encode long-term memories (Bourne and Harris, 2007, 2008; Kasai et al., 2003; Matsuzaki, 2007; Matsuzaki et al., 2001, 2004; Yang et al., 2009).

Spines are often classified according to type. Spine type is considered to play a role in information storage. Mushroom spines, spines with constricted necks and heads exceeding 0.6 microns in diameter (Bourne and Harris, 2007, 2008; Harris et al., 1992) are considered memory spines and involved in long-term memory storage. Mushroom spines can form strong synapses due to large postsynaptic densities (PSD) that accommodate greater numbers of AMPAR (Ashby et al., 2006; Bourne and Harris, 2007, 2008; Ganeshina et al., 2004; Harris et al., 1992). Thin spine, spines with a constricted neck and small head (Bourne and Harris, 2007, 2008; Harris et al., 1992) are considered learning spines and are involved in transient events. Thin spines are flexible, responsive to changes in synaptic activity levels and have smaller PSDs which contain fewer AMPAR but many NMDAR (Ashby et al., 2006; Bourne and Harris, 2007, 2008; Farris et al., 2001; Ganeshina et al., 2004; Harris et al., 1992; Hübener and Bonhoeffer, 2010).

A number of studies have used the Golgi method to investigate structural changes associated with memory formation (Hoh et al., 2003; Knafo et al., 2004; Leuner et al., 2003; Restivo et al., 2006, 2009; Vetere et al., 2011). The Golgi method, originally named the Black Reaction was developed by Camillo Golgi in 1873 (Golgi, 1873). The invention of the Golgi method marked a huge advancement in the field of neuroscience, largely thanks to Santiago Ramon y Cajal, who refined the technique and popularized its use (Cajal, 1888, 1909). The Golgi technique impregnates neurons at random in their entirety, staining cell bodies as well as all of their processes, permitting the examination of neuronal morphology. With entire neurons stained, it became possible to quantitatively examine a number of morphological characteristics such as dendritic length, spine number and more. Curiously, and fortunately, the Golgi method stains only a small percentage of nerve cells (estimates range between 1-5\% (Milatovic et al., 2010; Spacek, 1989). This is one of the great advantages of the Golgi method: if every neuron were to become impregnated and stained with Golgi, it would be impossible to trace a single neuron from its beginning to end amongst all the processes from other neurons. The seemingly random staining seen in the Golgi method provides the opportunity to examine individual neurons. Even presently, it is not well understood why some neurons become impregnated with Golgi solution and why some do not. Some factors suggested to play a role include the functional or metabolic state of a neuron at the time of Golgi fixation or mechanical injury from tissue processing rendering a neuron more susceptible to impregnation (Pannese, 2010; Spacek, 1989). The Golgi-Cox method used 
in this study is a variation on the original Golgi method. This variation uses potassium dichromate and mercuric chloride and is well suited for analyses in rats (Gibb and Kolb, 1998; Glaser and Loos, 1981). The Golgi-Cox method is considered well suited for the purposes of studying dendritic morphology (Buell, 1982; Ranjan and Mallick, 2010).

\subsection{Consolidation}

The fragility of new memories and their vulnerability to disruption has been well documented (Alberini et al., 2006; Dudai, 2004; McGaugh et al., 2000; Muller and Pilzecker, 1900; Squire and Alvarez, 1995; Squire and Barondes, 1972). The term consolidation refers to the postacquisition stabilization of long-term memory (Dudai, 2004). This term was first coined by Muller and Pilzecker (1900), who made the famous discovery that a memory representation takes time to stabilize. The term consolidation is now widely used to describe the variety of processes whereby initially labile, fragile memory traces stabilize over time and become permanently stored. The mechanisms by which consolidation proceeds, the time course and the ultimate site of long-term memory storage are still debated. Whatever these specific mechanisms are, they allow for the permanent storage of long-term memories to persist across the lifetime.

Consolidation refers to modifications at the synaptic level and reorganization at the systems level (Dudai, 2000, 2004, 2012; Frankland and Bontempi, 2005; McGaugh et al., 2000; Squire and Kandel, 1999). Synaptic consolidation, also termed local or cellular consolidation, is the quicker of the two types of consolidation and occurs in the hours following acquisition. Synaptic consolidation refers to synaptic growth or remodelling, the induction of signalling cascades and second messenger systems, gene expression alterations, and ultimately, modifications that alter synaptic dynamics in circuits important for encoding a memory (Alberini et al., 2006; Dudai, 2004, 2012; Kandel, 2001). Interference with any step of this process via behavioural, drug, or lesion manipulations can impair memory consolidation (Davis and Squire, 1984; Dudai, 2004; Frankland and Bontempi, 2005; Laroche and Davis, 2000; Squire and Barondes, 1972). This process underlies the morphological manifestations that occur during consolidation of a labile memory to a stable, persistent memory. Simply put, synaptic consolidation may be thought of as synaptic stabilization.

Systems consolidation is a much longer, more gradual process of reorganization of brain regions and circuits supporting memory (Dudai, 2004, 2012; Frankland and Bontempi, 2005; Kassardjian et al., 2005; McGaugh, 2000). Systems level consolidation refers to a slow and gradual process of reorganization and rearrangement in the brain circuits which support the storage of memories, shifting the storage of memories from an initial encoding circuit to a circuit for long-term storage (Alberini et al., 2006; Dudai, 2004, 2012; Frankland and Bontempi, 2005; Kandel, 2001; McGaugh et al., 2000). 


\subsubsection{Models of Consolidation}

\section{Standard Model of Consolidation}

Literature stemming from memory impairments associated with temporally graded retrograde amnesia led to the development of the Standard Model of Consolidation (SMC) (Squire and Alvarez, 1995). The SMC posits that as consolidation of a memory progresses, hippocampal involvement gradually disengages (McClelland et al., 1995; Squire and Alvarez, 1995). The SMC hypothesises information initially registered by the neocortex is integrated by the hippocampus and related structures, which acts as a preliminary assimilation center to form a memory trace (McClelland et al., 1995; Scoville and Milner, 1957; Squire and Alvarez, 1995). Over time, as the memory is rehearsed or replayed, extra-hippocampal sites become synchronized and coordinated such that they form cortico-cortical connections that become independent from the hippocampus (Dudai, 2004; Frankland and Bontempi, 2005; Squire and Alvarez, 1995; Winocur et al., 2010). The hippocampus acts as a support system during this passive process. In this way, memories acquired recently are dependent upon the hippocampus while remote memories have formed strong neural connections outside of the hippocampus and are stored in these extra-hippocampal areas, indicating a temporary role for the hippocampus in memory (Dudai, 2004; Frankland and Bontempi, 2005; McClelland et al., 1995; Scoville and Milner, 1957; Squire and Alvarez, 1995; Winocur et al., 2010).

The Standard Model is not universally supported. A major criticism is that it does not describe a mechanism whereby the transfer of memory from hippocampus to extra-hippocampal sites is accomplished (Nadel and Bohbot, 2001; Nadel et al., 2007). The theory taken in its most literal sense, defines memories as becoming independent from the hippocampus. If this were the case, hippocampal activation would not be expected to occur during retrieval. However, the hippocampus has been shown to be necessary for, and activated during, remote memory retrieval (Bolhuis and Stewart, 1994; Broadbent et al., 2006; Clark et al., 2005a,b, 2007; Epp et al., 2008; Martin et al., 2005; Mumby et al., 1999; Squire, 2004; Sutherland et al., 2001; Teixeira et al., 2006; Winocur et al., 2005a). Revisions to the SMC have justified this by suggesting it is not the retrieval of memories causing the activation, rather the encoding of new memories which require the hippocampus and its activation (Nadel et al., 2007). Another critique of the SMC is that the model does not distinguish between episodic and semantic memories. Retrograde amnesia has been noted in many cases to vary with memory type (see (Moscovitch et al., 2006, 2005). Retrograde amnesia resulting from medial temporal lobe lesions may be virtually ungraded for autobiographical/episodic memories, while amnesia for semantic memories is generally less extensive. The model does not account for how or why episodic memories become more semantic as they age (details are lost and memories become more gist-like), a major criticism of the standard model (Moscovitch et al., 2006, 2005; Nadel and Moscovitch, 1997, 1998). In fact, there is question as to whether all types of memory undergo systems level consolidation. Some suggest 
vivid episodic type memories may never become completely independent from the hippocampus (Nadel and Moscovitch, 1997; Winocur et al., 2010).

\section{Multiple Trace Theory}

Similar to the SMC, the Multiple Trace Theory (MTT), proposed by Nadel and Moscovitch (1997), suggests that the hippocampal complex encodes information that is attended to and binds the neocortical neurons that represent the experience into a memory trace (Moscovitch et al., 2005). The MTT distinguishes between episodic and semantic memories (Moscovitch et al., 2006, 2005; Nadel and Moscovitch, 1997, 1998). The MTT postulates autobiographical (episodic) memories always rely on the hippocampus, regardless of their age. In this respect, memories rich in detail and vividness permanently rely upon the hippocampus, suggesting that autobiographical (episodic) memories will always depend on the hippocampus, regardless of their age. A memory that had lost its vividness and detail, or become semanticized, would disengage from the hippocampus, and become dependent on the neocortex (Moscovitch et al., 2006). According to the MTT, there is no prolonged consolidation process as described by the SMC. Instead, MTT posits that each time an episodic memory is retrieved, a new trace in the hippocampus is created (Moscovitch et al., 2006, 2005; Nadel and Moscovitch, 1997, 1998; Nadel et al., 2007). This suggests that older memories are represented by more traces than recent memories, and are thus less vulnerable to disruption from damage. Older memories would be able to withstand hippocampal damage better than recent memories because the greater number of traces related to the older memory would increase the likelihood of some spared traces following damage. Thus, large and extensive lesions of the hippocampus would be required to disrupt them. Newer memories would be more vulnerable to hippocampal damage than older memories, because fewer traces would be associated with them. In this way, MTT accounts for temporally graded retrograde amnesia (Nadel et al., 2007). However, research has not consistently provided evidence to support this hypothesis, causing some to question the validity of the model (Bright et al., 2006; Broadbent et al., 2006; Clark et al., 2005b; Haijima and Ichitani, 2008; Kopelman et al., 2003; Lehmann et al., 2007; Martin et al., 2005; Quinn et al., 2008; Ramos, 2008, 2009; Sutherland and Lehmann, 2011; Sutherland et al., 2008).

\section{Cognitive Map Theory}

As with the SMC and MTT, Cognitive Map Theory (CMT) claims hippocampal and cortical neurons interact to form memory traces initially (Burgess et al., 2002; O'Keefe and Nadel, 1978). However, CMT does not differentiate between recent and remote memories. CMT is centered on the idea that the hippocampus is necessary for forming, retaining and retrieving allocentric spatial representations, regardless of their age (Burgess et al., 2002; Gomez et al., 2012; Moscovitch et al., 2005; Nadel and Moscovitch, 1998; O'Keefe and Nadel, 1978). CMT suggests that allocentric spatial representations provide the context for 
episodic events and focus on this idea in memory storage (Burgess et al., 2002; Moscovitch et al., 2006). Hippocampal damage, suggested by CMT, should result in impaired retrieval of spatial and episodic memories, regardless of recency or remoteness. Serious concerns have arisen about the CMT based on clinical observations and neuroimaging studies, which have shown that the hippocampus is not critical for retrieval and retention of remote spatial memories (even allocentric spatial memories) (Rosenbaum et al., 2005, 2000, 2001; Teng and Squire, 1999).

\subsection{Memory Dynamics}

Despite disagreement among consolidation theories, several lines of evidence indicate a dynamic role of the hippocampus in memory storage as a memory ages. Similarly, the role of the prefrontal corex (PFC), specifically the ACC, is suggested to change as a memory matures. Largely, though not exclusively, the hippocampus plays a significant role in recent memory, but disengages as a memory ages. The ACC becomes an increasingly important node as a memory becomes more remote. Overall, there appears to be a reorganization of structures involved in memory storage.

\subsubsection{Roles of the Hippocampus and Prefrontal Cortex}

There are two primary theories that account for the involvement of the PFC in remote, but not recent memories. Frankland and Bontempi (2005) suggest memories initially acquired by the hippocampus become consolidated in cortical areas remotely, such that cortical areas can function independently from the hippocampus at remote time points. This theory suggests the involvement of the PFC is dependent upon the age of the memory. Rudy et al. (2005) suggest the involvement of the PFC is not dependent upon the age of the memory, but the strength of the memory. They hypothesize that activation of the PFC results from failure to access a degraded, or weak, memory trace, and that activation of the PFC can increase activity in brain areas responsible for the memory trace. This theory implies that a weak, recent memory would require PFC activation, while a strong, remote memory would not. Regardless of which theory is correct (if either are), evidence supporting a reorganization of brain structures involved in memory storage exists in both humans and animal models.

\subsubsection{Memory Organization}

\section{Human Studies}

Human investigations into the effect of focal medial temporal lobe or cortical lesions are limited to the number of individuals that present with damage to the area of interest. Functional neuroimaging results complement lesion studies nicely. Though neither can provide a complete understanding of memory storage dynamics, their combination allows for greater insight into the processes at 
play.

A great deal of human research supports the idea of a time limited role for the hippocampus in memory storage (Barr et al., 1990; Bayley et al., 2005, 2003, 2006; Corkin, 1984; Kapur and Brooks, 1999; Kirwan et al., 2008; Manns et al., 2003; Marslen-Wilson and Teuber, 1975; O'Connor et al., 1992; Reed and Squire, 1998; Rempel-Clower et al., 1996; Rosenbaum et al., 2000; Scoville and Milner, 1957; Squire et al., 2001; Teng and Squire, 1999; Zola-Morgan et al., 1986). Evidence from these human studies suggests discrete hippocampal damage leads to severe anterograde amnesia and temporally graded retrograde amnesia, where recent memories are affected but remote memories are spared.

Recall patient H.M. who, at the age of 27 , underwent bilateral removal of his medial temporal lobes to treat the incapacitating seizures he suffered when all other therapies failed (Milner, 1962; Scoville and Milner, 1957). Following the removal of his medial temporal lobes, H.M. suffered from severe anterograde and temporally graded retrograde amnesia for declarative memories. The anterograde amnesia rendered him unable to commit new declarative information to long-term memory storage, while the temporally graded retrograde amnesia impaired his recent memory, but left his remote memory, for the most part, intact. This seminal case study implicated a transient role for the hippocampus in memory storage. H.M. was able to recall memories from his childhood, but was unable to recall memories in the few years leading up to his surgery, suggesting these more recent memories were still to some degree dependent on hippocampal function, while more remote childhood memories were increasingly independent from the hippocampus. For example, reports of H.M. described his inability to remember and locate his new home address to which he moved following his surgery (Milner et al., 1968; Scoville and Milner, 1957). However, he remembered the location of the home he had lived in before his operation. H.M. did not recognize neighbours or friends with whom he was acquainted with following his surgery, but remembered those he knew before his surgery (Milner et al., 1968).

As mentioned earlier, patient R.B. suffered an ischemic episode resulting in bilateral damage to the entire CA1 field of the hippocampus. Analyses demonstrated normal cognitive function on a variety of tests, with the exception of memory (Zola-Morgan et al., 1986). Patient R.B. exhibited profound anterograde amnesia, but little, if any, retrograde amnesia, suggesting a time limited role of the hippocampus in memory function. Patient E.P. had extensive bilateral medial temporal lobe damage that rendered him unable to acquire new information, including spatial memories. However, E.P. was unimpaired in his ability to recall remote spatial memories (Teng and Squire, 1999), contributing evidence to support the idea that remote spatial memories may be stored outside of the medial temporal lobes. E.P. was asked to navigate around the area he grew up in but had moved away from more than 50 years ago. E.P. was able to construct familiar routes, novel routes and point out landmarks from this area, as well or better than age-matched controls familiar with but who had also moved away from the area long ago. In stark contrast, E.P. demonstrated no knowledge of his current neighbourhood. He could not form a response when 
asked to perform the same tasks in the neighbourhood in which he currently resided, one that he had moved to following his surgery.

Kapur and Brooks (1999) reported the results of detailed memory testing on two patients with discrete bilateral damage limited to the hippocampus. The results from these two cases revealed mild retrograde amnesia limited to a few years prior to hippocampal damage, and severe anterograde amnesia, supporting the idea of a time-limited role for the hippocampus in memory storage. Manns et al. (2003) provided further support for a time-dependent role of the hippocampus in memory storage. Patients with bilateral damage limited to the hippocampal region were examined. Results suggested these patients suffered from temporally graded retrograde amnesia from years just prior to hippocampal damage, while their remote memory remained intact.

Not all evidence from human studies supports the idea of a time-limited role for the hippocampus in memory storage. There exists a wealth of research that suggests a seemingly permanent role for the hippocampus in memory (Barr et al., 1990; Bright et al., 2006; Cermak and O'Connor, 1983; Chan et al., 2007; Cipolotti et al., 2001; Damasio et al., 1985; Hirano and Noguchi, 1998; Kartsounis et al., 1995; Kopelman et al., 1999; Noulhiane et al., 2007; O'Connor et al., 1992; Sanders and Warrington, 1971; Steinvorth et al., 2005; Tulving et al., 1988; Victor and Agamanolis, 1990; Viskontas et al., 2000; Warrington and McCarthy, 1988).

S.S. presented with bilateral temporal lobe damage. He was able to retain information in his working memory, but was not able to retrieve such information at a later time, suggesting he could not transfer material into his long-term memory (Cermak and O'Connor, 1983). Analysis of his remote memory revealed a significant impairment in the ability to recall autobiographical events from the recent or remote past, indicating a time-independent role of the hippocampus in such memories. Cipolotti et al. (2001) examined patient V.C. who presented with markedly shrunken hippocampi, but normal EC volume. V.C. demonstrated profound, extensive, ungraded retrograde amnesia for the recall of episodic events. Steinvorth et al. (2005) examined two amnesic patients with bilateral medial temporal lobe lesions. Episodic and semantic remote memory tests were evaluated. Results from these tests suggested the medial temporal lobes played an important role in episodic memories in the form of autobiographical memory, regardless of the age of the memory. Findings suggested remote semantic memories did not rely on medial temporal lobe function.

Maguire et al. (2001) failed to show evidence of differences in age-related activity in the hippocampus as assessed by functional magnetic resonance imaging (fMRI). Noulhiane et al. (2007) examined autobiographical memory across the lifespan in patients who had received a left or right medial temporal lobe resection. Their findings indicated impaired autobiographical memories covering the entire lifespan in patients with medial temporal lobe resections. Moscovitch et al. (2006) suggested hippocampal function was required for detailed episodic and spatial memories regardless of age. These researchers stressed the importance of differentiating between episodic and semantic memory and used the Multiple Trace Theory to help explain their hypotheses. Rosenbaum 
et al. (2008) examined autobiographical events in amnesic patients with varying levels of hippocampal and neocortical damage. Their findings suggested episodic autobiographical memory depends largely on hippocampal function in a time independent manner. Winocur et al. (2010) reviewed a large body of human and animal research investigating the transformation hypothesis, which focuses on a few key distinctions from the standard model of consolidation. They list a number of studies where hippocampal damage did not result in temporally graded retrograde amnesia. They presented evidence that suggested hippocampal-mediated memories differ from extra-hippocampal mediated memories, and put forth an argument stressing the differences between hippocampalmediated memories and cortical-mediated memories.

Human literature on the cortical contribution to memory storage is not abundant. Takashima et al. (2006) examined the progression of consolidated memories in human subjects over a three-month time period. fMRI findings suggested hippocampal activity decreased as memory aged, while ventral medial prefrontal region activity increased. Takashima et al. (2007) found similar findings in a future study. Woodard et al. (2007) used fMRI to examine temporally graded signal differences in cortical areas. Results revealed the ACC was activated in response to both recent (5-10 years) and remote stimuli (40-50 years), with no significant difference in activation between the two. These findings supported a role for the ACC in recalling both recent and remote stimuli.

Söderlund et al. (2012) examined neural correlates when retrieving autobiographical memories. The hippocampus was bilaterally activated regardless of the age of the memory. Interestingly, imaging results showed continued connectivity between the hippocampus and neocortical structures and indicated the hippocampus had different functional connections to cortical regions when retrieving recent compared to remote events. Similarly, Harand et al. (2012) examined neural activity at 3 days and 3 months following initial learning. Data indicated that hippocampal activity remained stable over time for episodic memories, but decreased when initially episodic memories became semantic over time (memories lost contextual richness and detail). Therefore, these results suggested the retrieval of episodic memories relies on hippocampal activity regardless of the age of the memory. Activity in prefrontal cortical areas, notably the ACC, was found at both recent and remote time points. See tables 1.1 and 1.2 for a summary of selected human studies.

\section{Animal Models}

A large body of research from primates and rodents exists supporting a timelimited role of the hippocampus in memory storage (Anagnostaras et al., 1999; Cho et al., 1993; Cho and Kesner, 1996; Clark et al., 2002; Debiec et al., 2002; Gaskin et al., 2009; Glenn et al., 2003; Izquierdo et al., 1997; Kim et al., 1995; Kim and Fanselow, 1992; Kubie et al., 1999; Laurent-Demir and Jaffard, 1997; Maren et al., 1997; Maviel et al., 2004; Quillfeldt et al., 1996; Quinn et al., 2008; Ramos, 1998, 2009; Remondes and Schuman, 2004; Ross and Eichenbaum, 2006; Shimizu et al., 2000; Squire et al., 2004; Takehara et al., 2003, 2002; Tse et al., 
2007; Vnek and Rothblat, 1993; Ward et al., 1999; Winocur, 1990; Winocur et al., 2001; Zola-Morgan and Squire, 1990). Mounting evidence suggests the ACC becomes a critical node in memory storage at remote time points after encoding (Bonaccorsi et al., 2013; Bontempi et al., 1999; Ding et al., 2008; Frankland et al., 2004; Gafford et al., 2013; Holahan et al., 2007; Lopez et al., 2012; Maviel et al., 2004; Restivo et al., 2009; Teixeira et al., 2006; Vetere et al., 2011; Weible et al., 2012).

Zola-Morgan and Squire (1990) tested monkeys on an object discrimination task, learned prior to surgery, at recent and remote time points following bilateral lesion of the hippocampus. Control monkeys showed normal forgetting; remembering recently learned objects better than remotely learned objects. Monkeys that received hippocampal lesions were severely impaired on tests of recent memory, while they performed as well as controls on tests of remote memory and significantly better on tests of remote compared to recent memory, suggesting a time-limited role for the hippocampus in memory storage. Anagnostaras et al. (1999) used a within-subjects design where rats had both a recent and remote contextual fear memory. Rats that received dorsal hippocampal lesions exhibited a specific impairment in recent, but not remote, contextual fear memory, indicating memory disengagement from the hippocampus over time.

An influential study by Bontempi et al. (1999) provided convincing evidence of a reorganization of neuronal structures involved in memory storage over time. Non-invasive functional brain imaging was used to examine metabolic activity by examining (14C) 2-deoxy-glucose uptake in 74 brain regions in mice. Memory was tested using a spatial discrimination task (RAM) with testing intervals of 5 (recent) or 25 (remote) days following initial training. Results showed significantly lower levels of metabolic activity in the hippocampus in mice tested on retention remotely compared to mice tested recently. Remotely tested mice displayed increased levels of metabolic activity in the frontal, anterior cingulate and temporal cortices, indicating a reorganization of brain circuitry underlying remote memory storage. Additionally, remote retention tests included mice tested in a different room and different maze as well as mice tested in the same room with a different reward location. Mice in both these situations showed high levels of metabolic activity in the hippocampus. These findings showed that a novel context or novel reward location caused a reactivation of hippocampal metabolic activity. Combined, these results support the idea of systems level consolidation and the gradual disengagement of the hippocampus in memory storage over time, supporting the idea that the decline in hippocampal activity seen in the original remote retention group is due to the retrieval of remotely stored information.

Frankland et al. (2001) provided further support of a reorganization of structures underlying remote memory storage. These researchers elucidated some of the molecular processes underlying the storage of remote memories in cortical networks. They showed that mice with an $\alpha$-CaMKII+/- mutation had impaired cortical LTP (but not impaired hippocampal LTP) and were impaired on two hippocampal-dependent tasks at long-term retention intervals (10-50 
days), but not short-term retention intervals (1-3 days). As a follow up to this study, Frankland et al. (2004) presented a series of experiments which investigated the expression of activity-dependent genes in specific cortical areas and the effect of temporary inactivation of cortical areas, in $\alpha$-CaMKII $+/-$ mutants, using contextual fear conditioning at recent (1 day) and remote (36 days) time points. Two activity-dependent genes were examined: zif-268 (an immediate early gene) and c-Fos. Wildtype mice showed increased expression of these genes in the ACC, infralimbic cortex, prelimbic and temporal cortex after remote memory tests. $\alpha$-CaMKII+/- mice, that were impaired on tasks at remote retention intervals, did not show increased expression of activity-dependent genes in these cortical areas. Lidocaine was injected into the prelimbic and anterior cingulate cortex to determine the effect a localized, transient, inactivation of these areas would have on memory. Inactivation of the prelimbic cortex caused no discernible disruption in memory function at recent or remote time points tested. Inactivation of the ACC caused disruption of remote, but not recent, contextual fear memory, suggesting the ACC plays a vital role in remote memory storage and/or retrieval processes. Furthermore, levels of zif-268 in the CA1 of the hippocampus were reduced below control levels in wildtype mice following tests of remote memory, suggesting hippocampal inhibition may occur during remote memory processing.

Maviel et al. (2004) provided evidence of a time-limited role for the hippocampus in memory consolidation. These researchers showed an increase in c-fos and zif268 expression as well as structural changes including synaptogenesis and laminar reorganization in the ACC in remote (30 days) but not recent ( 1 day) memory tests of a baited RAM task. These researchers investigated the effect of an inactivation of the ACC and found that local inactivation disrupted remote memory retention, suggesting a vital role for the ACC in remote memory storage and/or retrieval. Teixeira et al. (2006) used c-fos expression patterns as well as pharmacological blockade to show the engagement of the ACC in processing remote (30 days), but not recent (1 day) spatial memories. They also showed that the hippocampus was necessary for spatial memory processing regardless of the age of the memory finding no difference in c-fos expression patterns in the hippocampus at recent or remote time points. Ross and Eichenbaum (2006), showed a transitory role of the hippocampus in memory storage using a social transmission of food preference memory test. Evidence from these studies showed recent (1 day) but not remote (21 days) hippocampal damage produced behavioural deficits in this food preference task evidenced by recently damaged rats displaying a lack of preference in food choice.

Restivo et al. (2009) demonstrated hippocampal disengagement over time by examining dendritic spine growth using Golgi-Cox impregnation following contextual fear conditioning at recent (24 hour) and remote (36 days) time points. They found increased spine density on hippocampal neurons at the recent time point and increased spine density on ACC neurons at the remote time point. Furthermore, brains examined at the remote time point did not show increased spine density in the hippocampus. Interestingly, this pattern of spine density fluctuations was seen even when no memory test was given, suggesting these 
changes do not occur as a result of memory retrieval, but occur naturally as the memory ages. Restivo et al. (2009) also examined the effect of lesioning the hippocampus directly following conditioning. Hippocampal lesions resulted in impaired performance on a remote memory task and also prevented the growth of spines on ACC neurons at the remote time point. Vetere et al. (2011) examined whether interfering with spine growth in the ACC would disrupt memory consolidation. Mice were trained on a contextual fear conditioning task followed by interference with spine growth one day or 42 days later. Results revealed that interfering with spine growth immediately following contextual fear conditioning resulted in disrupted memory expression assessed one week later. These results suggest the ACC is a critical node involved in remote memory expression.

Neurophysiological support for a role of the ACC in remote memory comes from Weible et al. (2012) who recorded neurons in the ACC of mice that underwent an object memory task. Mice received a single exposure or repeated exposures to a pair of objects in an arena and were returned back to the arena following a delay of 6 hours or 30 days, with one of the objects removed. Caudal ACC neurons displayed absent-object responses in mice that had been repeatedly exposed to the objects during familiarization up to 30 days following the familiarization sessions. These results suggest caudal ACC neurons show electrophysiological correlates for consolidation and remote memory processes.

Studies which provide evidence displaying a flat, or ungraded, retrograde amnesia following hippocampal damage exist as well (Bolhuis and Stewart, 1994; Broadbent et al., 2006; Burwell et al., 2004; Clark et al., 2005a,b, 2007; Driscoll et al., 2005; Epp et al., 2008; Gaffan, 1993; Gaskin et al., 2003; Haijima and Ichitani, 2008; Koerner et al., 1996; Lehmann et al., 2007, 2013; Martin et al., 2005; Mumby et al., 1999; Riedel et al., 1999; Salmon et al., 1987; Sparks et al., 2011; Sutherland et al., 2008; Thornton et al., 1997; Weisand et al., 1996; Wiltgen et al., 2006; Winocur et al., 2005a,b). Similarly, conflicting results exist for the role of the ACC, with some research suggesting that inactivation of the ACC does not impair remote memory storage or retrieval processes (DeCoteau et al., 2009; Oswald et al., 2010).

Typically, ungraded amnesia in animal research arises when spatial memory is being tested, specifically when using the WM task (Bolhuis and Stewart, 1994; Clark et al., 2005b; Martin et al., 2005; Mumby et al., 1999; Mumby and Glenn, 2000; Riedel et al., 1999; Sutherland et al., 2001). Bolhuis and Stewart (1994) trained rats on a hidden platform water maze task then gave them sham lesions or lesions of the hippocampus 3 days or 14 weeks following the end of training. Rats that received hippocampal lesions spent significantly less time in the training quadrant compared to sham animals, and displayed a great deal of circling behaviour during the probe test. These behavioural deficits in hippocampal-lesioned animals were noted 3 days following the end of training as well as 14 weeks following the end of training, indicating the lack of a timedependent role of the hippocampus in memory storage.

Broadbent et al. (2006) trained rats on a hidden platform water maze task to examine the effects of reversible inactivation of the hippocampus. Rats were 
given a lidocaine or artificial cerebral spinal fluid (aCSF) injection into the hippocampus 4-5 hours or 30 days following training. Probe test analyses revealed rats given lidocaine injections spent significantly less time in the training quadrant and entered the target zone significantly fewer times compared to the aCSF injected rats, at both recent (4-5 hours) and remote (30 days) injection intervals. Furthermore, rats given a lidocaine injection recently, and retested two days after without injection, performed as well as controls on the water maze probe test. The results suggest the hippocampus is important in recent and remote spatial memory in the water maze task.

Clark et al. (2005b) suggested the reason research animals may show ungraded retrograde amnesia was because training procedures did not occur at an early enough age and training procedures did not permit rodents to learn the behavioural tasks sufficiently well. To this end, they began training rats at 21 days of age. Rats received 49 training sessions over 69 days. Hippocampal lesions were made 100 days following the completion of training. Probe test results indicated rats given hippocampal lesions showed no evidence of spared remote memory. Follow up studies from this group suggested hippocampal lesions resulted in performance deficits, responsible for the appearance of a flat, ungraded retrograde amnesia (Clark et al., 2007). Another possibility for the discrepancy seen between human and animal research when testing rodents on spatial tasks such as the water maze lies in the nature of the testing procedure. Human testing on measures of remote spatial memory, generally require an answer to the questions posed, simply stated verbally or depicted pictorially. These procedures are not possible when testing rodents. Instead, physical testing procedures must be used, which may depend on new learning to some degree. For example, to successfully complete the water maze task, rodents need to keep informed of their location in space relative to cues- this may require new learning.

Not all animal research supports a role for the ACC in remote memory. Oswald et al. (2010) presented results on trace eye blink conditioning in rabbits finding that ibotenic acid lesions to the ACC immediately following learning resulted in performance deficits. However, ibotenic acid lesions to the ACC one week following learning did not result in significant performance deficits, suggesting a role for the ACC in recently, not remotely acquired information. DeCoteau et al. (2009) examined long-term object-based memory on a go/no-go scene discrimination task. The effect of lesioning the ACC resulted in only a transient impairment in performance. Ross and Eichenbaum (2006) examined patterns of activation following training on a social transmission of food preference task. The experimenters demonstrated damage to the hippocampus 1 day, but not 21 days, following training impaired recall of this task. The experimenters found differences in c-fos activity in the ventral subiculum as well as in olfactory recipient regions, but failed to find differences in activation in other regions including the ACC. See tables 1.3 and 1.4 for a summary of selected animal studies. 


\subsubsection{Limitations}

Competing theories regarding memory consolidation and storage have encouraged disagreement concerning the roles of the hippocampus and cortical areas in such processes. The primary competing theories, the Systems Consolidation Model and the Multiple Trace Theory, each receive support from human and animal research. However, research in this field, from both human and animal studies, is flooded with inconsistencies. Following the human case studies and experimental animal studies introduced above, it is valuable to include a brief overview of some of the limitations present within the literature.

Human lesion research often samples from a pool of patients with damage that is not limited to a single area in the brain. Damage to the brain may result from a number of causes including trauma, infection, disease or surgery. Thus, patients often present with damage in many brain areas, making it difficult to determine lesion effects and to compile results across patients. Recent advancements in imaging technology allow for more accurate assessments of the extent and localization of brain damage. Techniques used previously may have over- or under-estimated the extent of damage in patients, leading to potentially flawed conclusions. In fact, recent research regarding the infamous case study of H.M. showed the extent of the medial temporal lobe resection was smaller than previously thought, revealing H.M. retained a significant portion of the hippocampus in both hemispheres (Annese et al., 2014). The assessments used to determine the extent of memory impairment in patients have progressed throughout the years. Assessments with greater specificity have been developed to more accurately reflect the memory deficits present in individuals. For example, tests that differentiate between semantic and episodic memory are now used. Prior to the idea that these different types of memory may be stored differently, careful analysis of these types of details were not taken. Additionally, older research may not have sufficiently examined remote memories by probing long enough into the past.

Animal research permits many manipulations not possible in human research. However, these manipulations are accompanied by inconsistencies. Methodological variances in experiments, such as differences in behavioural procedures and tasks, can influence experimental outcomes substantially. Memory is difficult to test in rodents because it requires a measure of behavioural performance to indicate memory. This complicates memory examination as it becomes difficult to determine whether neurological results result from the memory of an event, or from the performance of a task.

Animal research investigating the ACC in memory storage is a relatively new field. Historically, the ACC has been linked to higher cognitive functions such as conflict resolution, emotional processing and decision making (Aharoni et al., 2013; Allman et al., 2001; Bush et al., 2000; Cohen et al., 2000; Etkin et al., 2011; Gehring and Knight, 2000; Weissman et al., 2005), only recently has it become of interest to memory researchers. As such, the distinct subregions of the ACC under investigation may be of importance. The region of the ACC under examination may account for inconsistencies within literature. The 
rostral and caudal regions of the ACC may play different roles. The region of the ACC targeted in some of the experiments where ACC involvement was not found to be important in long-term memory, was more rostral than in studies whose results support a remote role of the ACC, suggesting anatomical differences in the ACC may be an important consideration (Weible, 2013). In spite of the accumulating evidence supporting a role of the ACC in remote memory, the precise role the ACC plays in facilitating remote memory is largely still unknown.

Evidence gathered from animal models of memory impairment is often used to help understand the human condition. Clearly, rodent and human brains are different. The cerebral cortex of a rat is approximately a thousand times smaller than that of humans (Uylings and Van Eden, 1990). Primates have also developed more complex cognitive functions, leading researchers to question whether rat brains contain comparable cortical areas with primates. In drawing functional and anatomical comparisons from research in rats to humans, it becomes challenging to determine homologous areas- such is the case in considering the ACC. It is important to consider the available data and literature in terms of the limitations it includes. The contradictions present may represent avenues worthy of further research.

\subsection{Objectives \& Hypothesis}

The underlying mechanisms associated with remote memory storage remain to be fully elucidated. How are these memories able to become stable, durable and persist for a lifetime? Brain regions of importance have been uncovered through case studies and animal research, but there is disagreement about the roles these areas play. Molecular markers of interest in memory consolidation and storage have been suggested, while structural changes are widely still believed to underlie memory storage. Given the great number of uncertainties in remote memory storage coupled with disagreement in the literature, the present thesis introduced a unique behavioural procedure to better characterize the mechanisms and regions involved. We hypothesized that increasing the processing demands placed upon the hippocampus would accelerate the time course of memory consolidation, and that structural changes at the synaptic and systems level would be visible at an accelerated timeframe. Furthermore, we suggest a continued involvement of the hippocampus in memory processes, regardless of memory age, but hypothesize a cortical region, the ACC, will become of increasing importance as consolidation progresses. To this end, Manuscript 1 reports on activational markers following the novel behavioural manipulation. Manuscript

2 examines structural correlates in the ACC and hippocampus following training on the behavioural procedure. Manuscript 3 examines the direct contribution of the ACC through transient inactivation following behavioural training. 


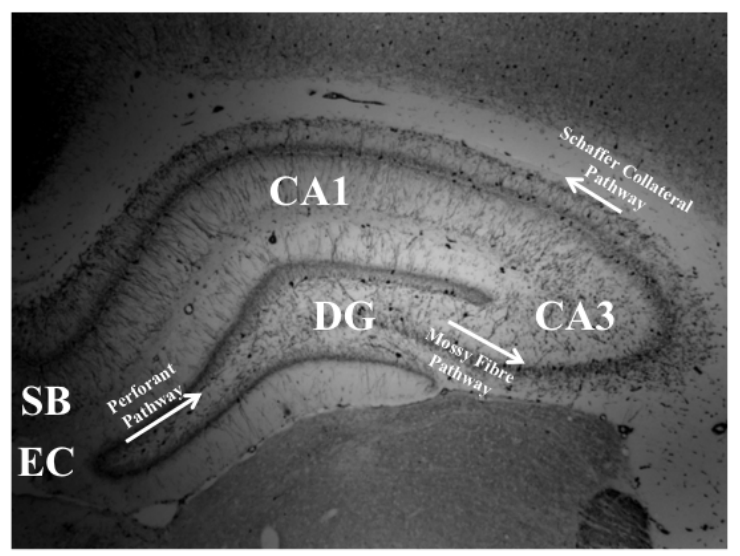

Figure 1.1: Hippocampal pathways of connectivity.The primary areas and networks within the rat hippocampus depicting the largely unidirectional flow of information. The EC provides the majority of synaptic inputs to the DG and CA3 through the Perforant Pathway. Granule cells from the DG predominantly project to the CA3 via the Mossy Fibre pathway. The major efferent connection from the CA3 projects to the CA1 via the Schaffer Collateral pathway. The CA1s major output is to the subiculum (SB) and EC. 


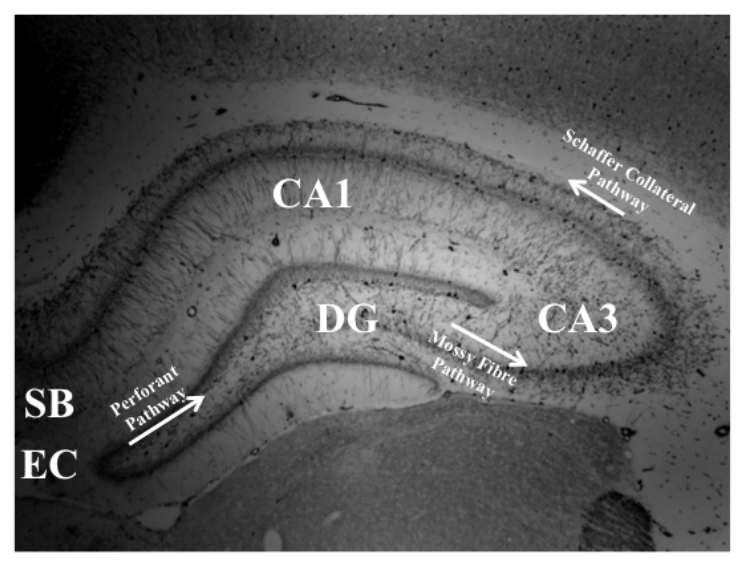

Figure 1.1: Hippocampal pathways of connectivity.The primary areas and networks within the rat hippocampus depicting the largely unidirectional flow of information. The EC provides the majority of synaptic inputs to the DG and CA3 through the Perforant Pathway. Granule cells from the DG predominantly project to the CA3 via the Mossy Fibre pathway. The major efferent connection from the CA3 projects to the CA1 via the Schaffer Collateral pathway. The CA1s major output is to the subiculum (SB) and EC. 


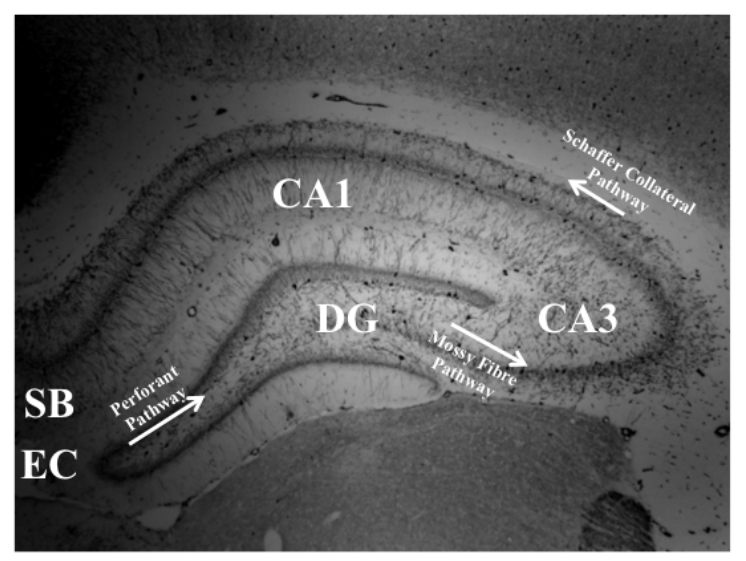

Figure 1.1: Hippocampal pathways of connectivity.The primary areas and networks within the rat hippocampus depicting the largely unidirectional flow of information. The EC provides the majority of synaptic inputs to the DG and CA3 through the Perforant Pathway. Granule cells from the DG predominantly project to the CA3 via the Mossy Fibre pathway. The major efferent connection from the CA3 projects to the CA1 via the Schaffer Collateral pathway. The CA1s major output is to the subiculum (SB) and EC. 


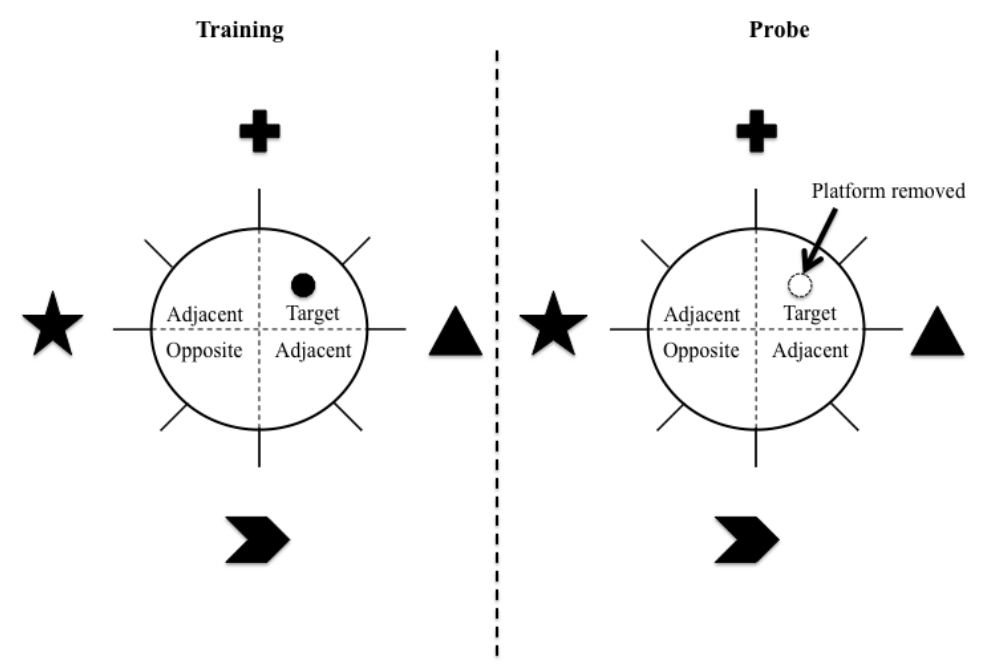

Figure 1.2: Illustration of the water maze task. 


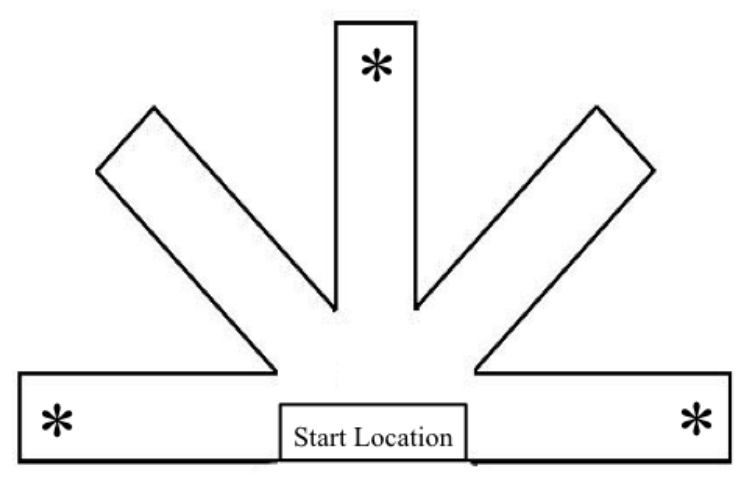

Figure 1.3: Illustration of the radial arm maze task. 


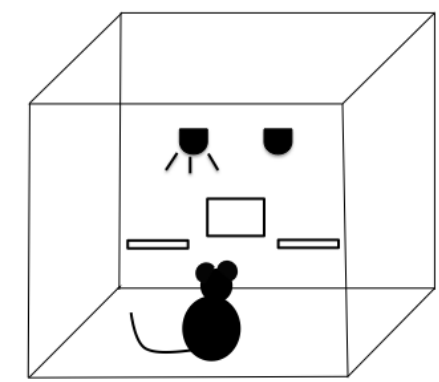

Figure 1.4: Illustration of the operant task. 


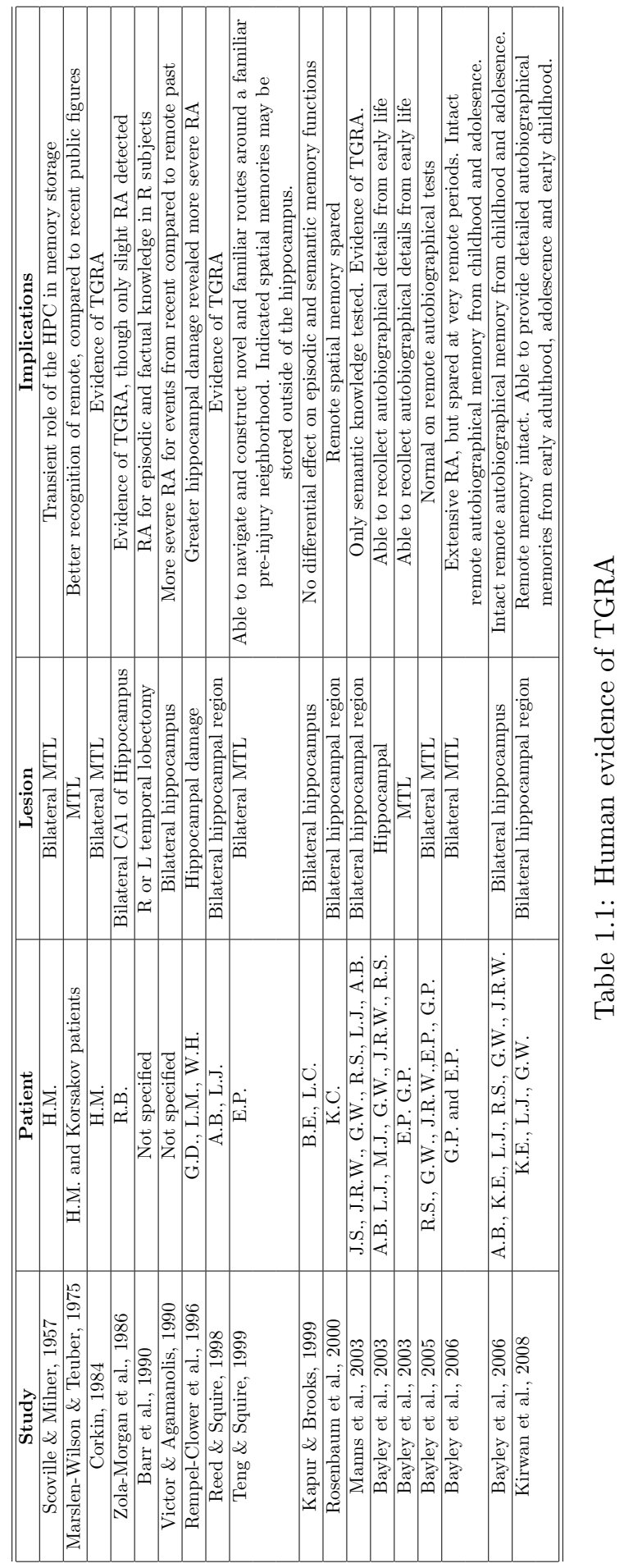




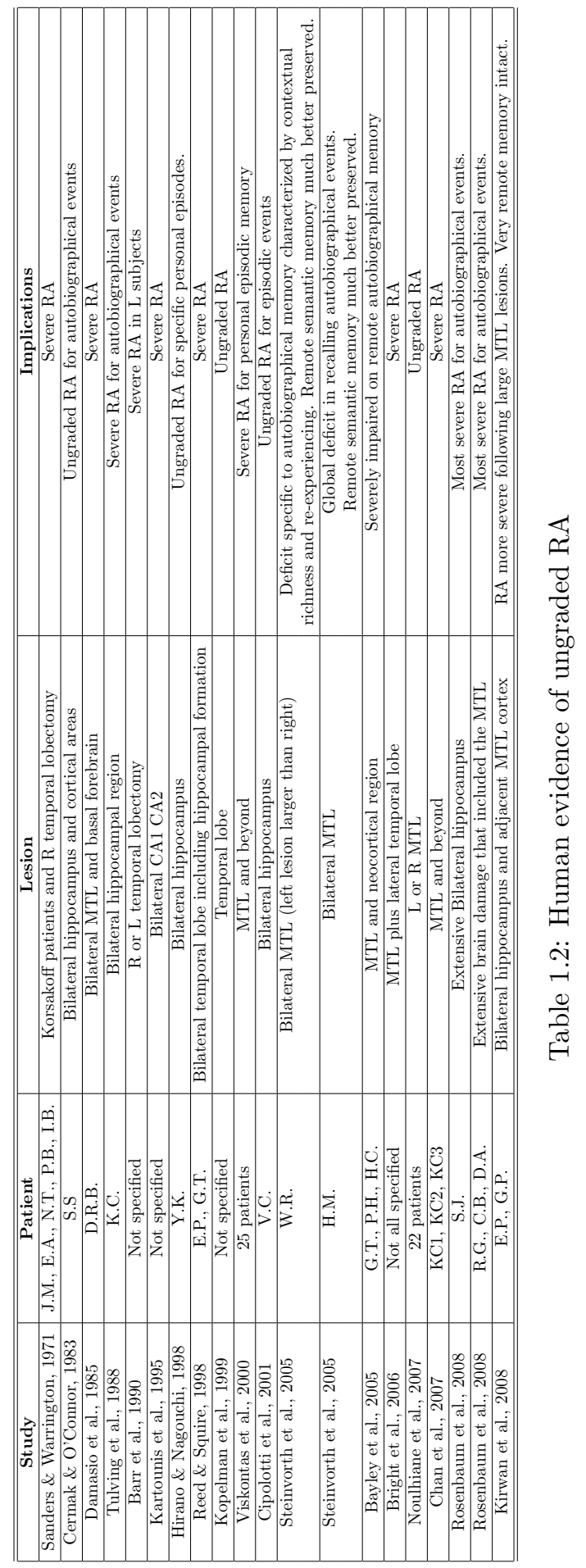




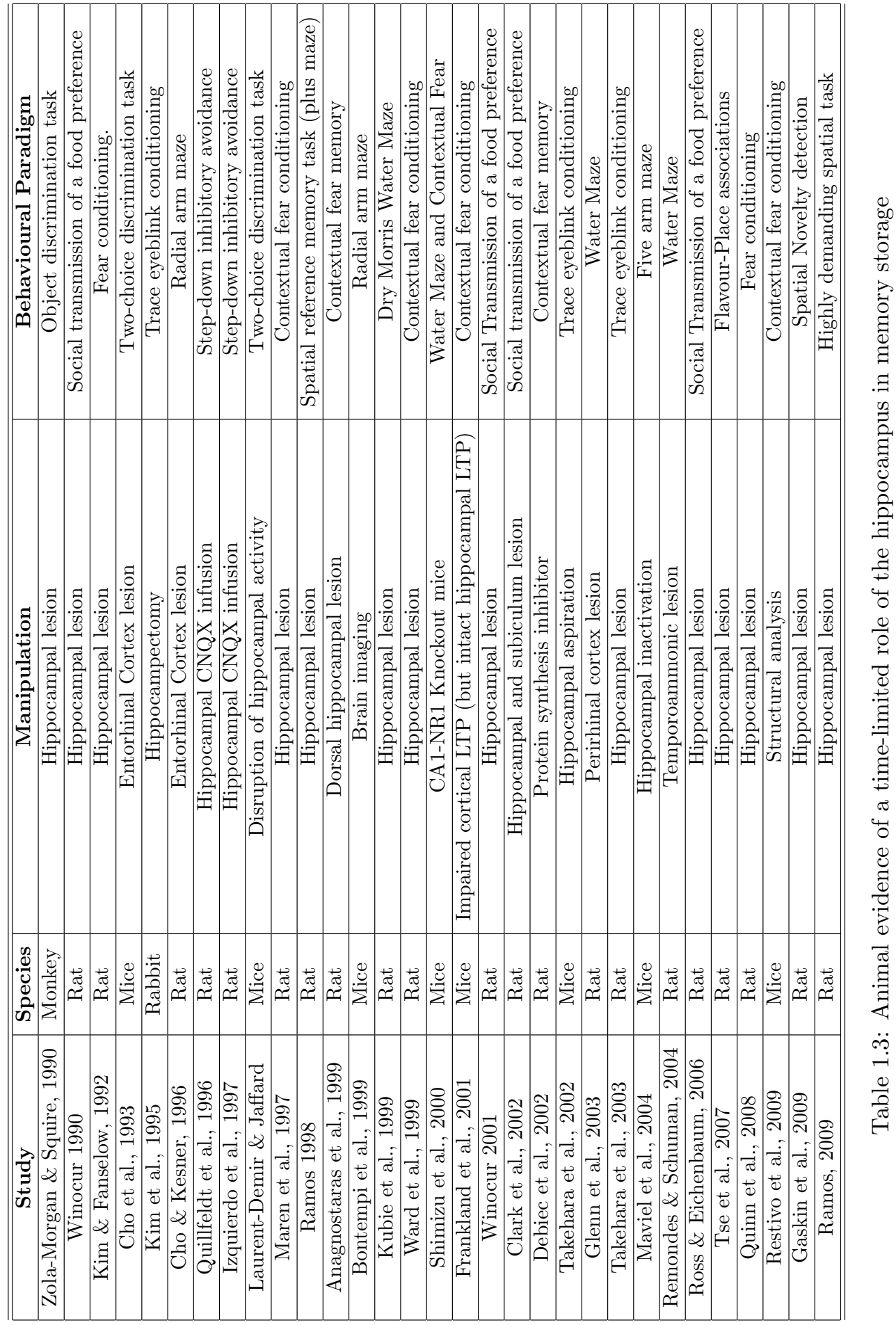




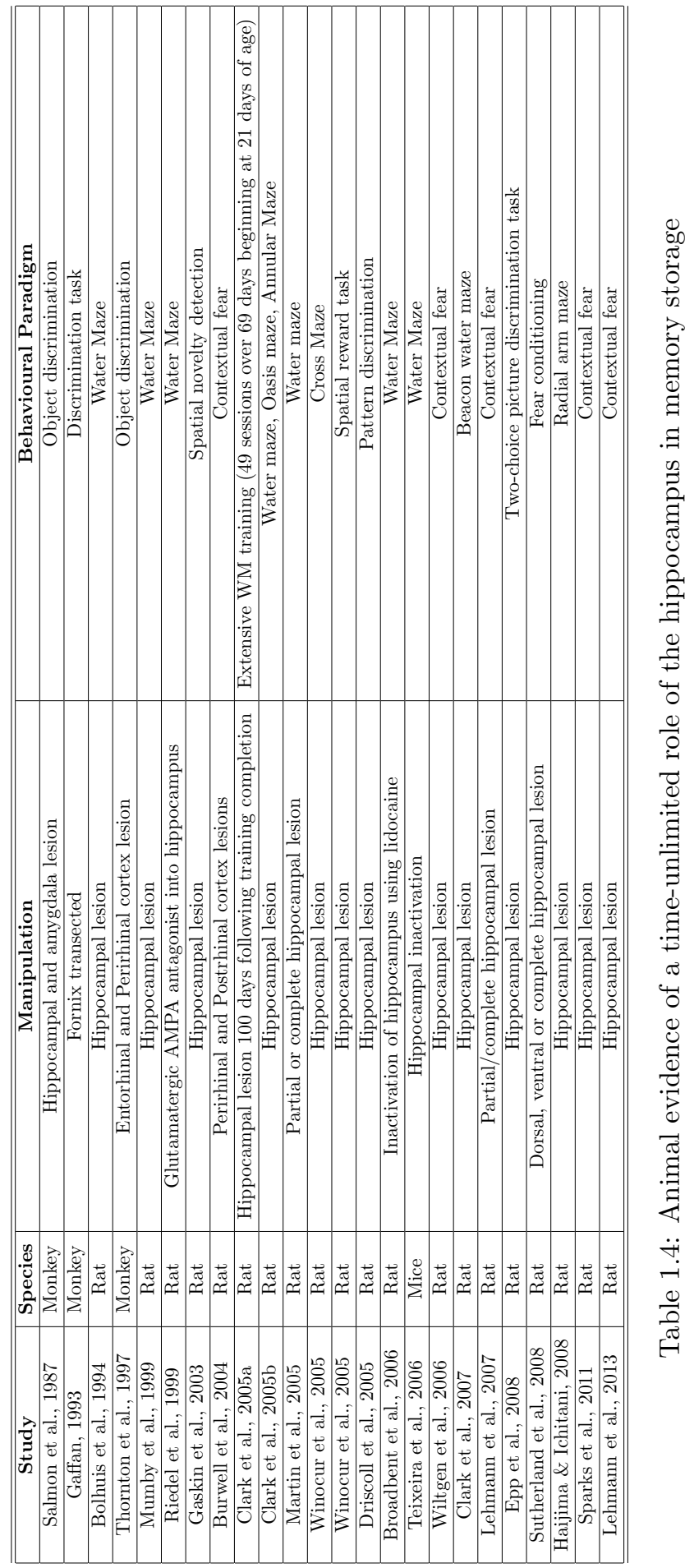




\section{Manuscript 1}

The factors that influence where and when a memory will be represented in a particular brain region are not well understood. Some evidence suggests the hippocampus only participates in recent memory processes and, as time progresses, the anterior cingulate cortex (ACC) plays a prominent role in remote memory processes. The present study sought to determine whether taxing the memory processing demands of the rat would influence the time course at which activation in the hippocampus and anterior cingulate cortex was observed during recent and remote memory processing. To examine this, a multiple memory behavioural procedure was used to investigate systems consolidation processes within the hippocampus and ACC by measuring protein expression in these two brain regions at recent and remote time points. 


\section{Chapter 2}

The use of sequential hippocampal-dependent and -non-dependent tasks to study the activation profile of the anterior cingulate cortex during recent and remote memory tests

B.C. Wartman \& M.R. Holahan

Neurobiology of Learning and Memory, 2013

106: 334-342. doi: 10.1016/j.nlm.2013.08.011

Department of Neuroscience, Carleton University

Keywords: remote memory; hippocampus; anterior cingulate; spatial memory; water maze; radial arm maze; multiple memories 


\section{$2.1 \quad$ Abstract}

Recent findings suggest that as time passes, cortical networks become recruited for memory storage. In animal models, this has been studied by exposing rodents to one task, allowing them to form a memory representation for the task then waiting different periods of time to determine, either through brain imaging or region-specific inactivation, the location of the memory representation. A number of reports show that 30 days after a memory has been encoded, it comes to be stored in cortical areas such as the anterior cingulate cortex. The present study sought to determine what factors, in addition to the passage of time, would influence whether memory retrieval was associated with cortical activation. To this end, rats were assigned to one of three behavioural groups: 1) Training on one hippocampal-dependent memory task, the water maze (WM); 2) Training on two, different hippocampal-dependent memory tasks, the WM followed by the radial arm maze; 3) Training on one hippocampal-dependent memory task (WM) followed by training on one non-hippocampal-dependent task, operant conditioning. After training, each group received a recent (2 d) or remote $(31 \mathrm{~d})$ water maze probe test. The group trained on two different hippocampal-dependent tasks and tested $2 \mathrm{~d}$ later, showed the strongest preference for the platform location during the probe test. This group also displayed a pattern of c-Fos staining in the anterior cingulate cortex similar to the pattern of staining observed in the remotely-tested groups and different from that seen in the other recently-tested groups. These results suggest the formation of multiple hippocampal-dependent memories accelerate the speed at which cortical network recruitment is seen and leads to enhanced behavioural performance in the recent term. 


\subsection{Introduction}

Consolidation is a naturally occurring process whereby a recently acquired memory becomes permanent over time. In 1900, Müller and Pilzecker (as described in (Lechner et al., 1999)) proposed that temporary reverberation of neural activity representing a memory or trace persists after an event and consolidation of the trace takes place during this period. The reverberating activity may promote synaptic changes thought to mediate more permanent memory representations (Hebb, 1955; McGaugh and Herz, 1972; Milner, 1957).

Current uses of consolidation refer to neural modifications that happen at the cellular and systems levels (Dudai, 1996, 2000, 2004; Frankland and Bontempi, 2005, 2006; McGaugh et al., 2000; McGaugh, 2000). Systems consolidation appears to be a gradual process whereby the permanent storage of a memory may ultimately be represented by neural circuits that are linked to, but independent from, the brain circuit that initially encoded the memory. In this view, memory representations show a shift in the balance of neural locus for long-term or remote storage (Dudai, 2009; Frankland and Bontempi, 2005, 2006; Kandel, 2001; Wiltgen et al., 2004).

Evidence from animal models suggests that memories initially encoded by the hippocampus become increasingly dependent on cortical areas at remote time points (Bontempi et al., 1999; Ding et al., 2008; Frankland et al., 2004; Holahan and Routtenberg, 2007; Kim and Fanselow, 1992; Maviel et al., 2004; Teixeira et al., 2006). In this view, the anterior cingulate cortex (ACC) has been implicated as a site for remote memory storage. Work has consistently shown evidence of increased activity in the ACC on tests for remote memory (Bontempi et al., 1999; Frankland et al., 2004; Lopez et al., 2012; Maviel et al., 2004; Teixeira et al., 2006; Weible et al., 2012). Inactivation of the ACC has been shown to hinder performance on remote memory tests (Frankland et al., 2004; Lopez et al., 2012; Maviel et al., 2004; Teixeira et al., 2006) and evidence has shown structural changes indicative of memory storage within the ACC at remote time points (Restivo et al., 2009; Vetere et al., 2011).

Evidence also exists supporting a hippocampal-centric view of memory storage, whereby damage to the hippocampus at any time after memory formation results in a flat, ungraded, retrograde amnesia (Broadbent et al., 2006; Clark et al., 2005a,b, 2007; Epp et al., 2008; Lehmann et al., 2007, 2013; Sparks et al., 2011; Sutherland et al., 2008). While various explanations have been put forth to address these differential findings (Sutherland and Lehmann, 2011; Sutherland et al., 2010; Winocur et al., 2010), the current study explored an alternate possibility to explain why a memory may come to be represented in extrahippocampal networks such as the ACC.

Systems consolidation models suggest that as time passes, there is a shift in the balance of neural activity from the hippocampus to the ACC in the representation of a remote memory. This has typically been studied by exposing rats to one task, allowing them to form a memory for the task, then waiting different

periods of time to assess the location of the memory representation based on neural activity. Some reports indicate 30 days after encoding, the ACC becomes 
a critical node in storing the memory (Bontempi et al., 1999; Ding et al., 2008; Frankland et al., 2004; Kim and Fanselow, 1992; Maviel et al., 2004; Teixeira et al., 2006), while other cases have reported the hippocampus remains engaged (Broadbent et al., 2006; Clark et al., 2005a,b, 2007; Epp et al., 2008; Lehmann et al., 2007, 2013; Sparks et al., 2011; Sutherland et al., 2008). We sought to determine if the shift in the balance of neural activity favouring the ACC could be influenced by the number of memories being sequentially processed. It was hypothesized that multiple hippocampal-dependent memories may impact the time course at which the ACC becomes part of a critical node in storing memory representations.

\subsection{Materials \& Methods}

\subsubsection{Subjects}

A total of 49 male Long Evans rats (190-250 grams) from Charles River, Quebec were used. Rats were housed individually in clear plastic cages (26 X 20 X 45 $\mathrm{cm}$ ) and given water ad libitum under a 12 hour light/dark cycle (lights on at 8:00 a.m.; rats tested during the light phase). Rats received no nesting material and no direct enrichment of any kind in their home cage environment. Efforts were made to minimize enrichment and experiences outside of those outlined in the experimental procedure to minimize the impact of housing variables. Food was restricted until rats reached $90 \%$ of their free-feeding baseline, which was maintained throughout the experiment. Prior to behavioural training, rats were given 5 chocolate pellets $(45 \mathrm{mg})$ in their home cage and handled for 5 minutes daily. Principles of laboratory animal care were followed and all procedures were conducted in accordance with the Canadian Council on Animal Care and protocols approved by the Carleton University Animal Care Committee.

\subsubsection{Apparatus}

\section{Water Maze (WM)}

The water maze was located in a room within the animal housing area. The opaque, white, polypropylene pool measured $155 \mathrm{~cm}$ in diameter and $60 \mathrm{~cm}$ in height and was filled to a depth of $37.5 \mathrm{~cm}$ with water that remained at approximately $21^{\circ} \mathrm{C}$. The platform was submerged $2 \mathrm{~cm}$ below the water surface and made from clear Plexiglas. Visual cues such as posters and geometric shapes were placed on the walls at distances of $48 \mathrm{~cm}, 188 \mathrm{~cm}, 54 \mathrm{~cm}$ and $104 \mathrm{~cm}$. The experimenter remained in the same position throughout all trials.

\section{Radial Arm Maze (RAM)}

RAM testing was done in a different room than WM testing located across the hall within the animal housing area. The maze was positioned $98.5 \mathrm{~cm}$ off the floor. Each arm measured $59 \mathrm{~cm}$ long and $11 \mathrm{~cm}$ wide. The distance between 
the ends of arms, where food (chocolate pellet- BioServe, New Jersey) reward was located was $32.5 \mathrm{~cm}$. Plastic inserts were located on the sides of maze arms to stop animals from jumping across arms. Visual cues such as posters and geometric shapes were located on the walls around the room. The experimenter remained in the same position throughout all trials.

\section{Operant Conditioning (OP)}

Rats were tested in groups of six using six operant chambers (Habitest Operant Cage, Coulbourn Instruments; 12 X 10 X 12 inches). Each chamber was housed in an insulated box to minimize external noise. Each chamber possessed a pellet dispensing system, two levers separated by a food hopper, a houselight, a grid floor and a three light-panel. OP training took place in a room outside of the animal housing area.

\subsubsection{Behavioural Procedure}

An overview of the behavioural procedure is shown in Figure 2.1. Rats were assigned to one of 3 behavioural groups: 1) Training on one hippocampaldependent task, the WM; 2) Training on two, hippocampal-dependent tasks, the WM followed by the RAM; 3) Training on one hippocampal-dependent task (WM) and training on one non-hippocampal-dependent task, OP. Sequential training on two tasks was separated by a 24 hour rest period. Following this training, rats received either a recent WM probe test $(8$ days after the end of WM training, $\mathrm{n}=25$ ) or a remote $\mathrm{WM}$ probe test (37 days after the end of WM training, $\mathrm{n}=25)$. Six experimental groups resulted: 1) WM:recent $(\mathrm{n}=8), 2)$ WM:remote $(\mathrm{n}=8), 3) \mathrm{WM} / \mathrm{RAM}$ :recent $(\mathrm{n}=8)$, 4) WM/RAM:remote $(\mathrm{n}=7)$, 5) WM/OP:recent $(\mathrm{n}=9)$ and 6) WM/OP:remote $(\mathrm{n}=9)$.

\section{Water Maze}

Rats received 5, 60 second training trials per day for 5 days with a different starting location for every trial within a day and randomized starting locations across days. The hidden platform was in a fixed location within and across days. Rats were placed in the pool facing the wall and given a maximum of 60 seconds to locate the hidden platform. Rats that did not find the hidden platform within 60 seconds were guided to the platform by the experimenter. All rats remained on the platform for 15 seconds then given a 15 second rest period in a holding cage before the next trial. All movement within the pool was tracked using HVS Image 2100 Tracking System (HVS Image, Buckingham, UK). Following the final trial of each day, rats were dried with a towel and placed in a holding cage on a heating pad in the housing room for 10-15 minutes after which they were returned to the home cage. 


\section{Radial Arm Maze}

Rats received one day of pretraining and 4 days of testing on the RAM. On the first trial of pretraining, chocolate pellets were located in the starting area, at the entrance to arms, within the arms as well as in the food holes located at the end of each arm. On trial 2 of pretraining, pellets were located within the arms and in food holes only. Trials 3-5 of pretraining had pellets located only in food holes.

Days 2-5 were testing days, where pellets were located in food holes at the ends of the 3 of the 5 arms. Baited arms were always the same for an individual rat but differed between rats. Each rat was given 5 trials per day. Trials were a maximum of 5 minutes each or ended when all food reward had been collected. Rats were placed in a holding cage for 30 seconds between trials while arms were re-baited. Performance on the maze was manually scored. Sessions were timed and correct and incorrect arm entries were recorded. An arm entry was defined as all 4 feet inside an arm.

\section{Operant Conditioning}

Rats in the WM/OP condition were trained to lever-press for chocolate pellets over 5 days, 30 minutes per day. Upon pressing the lever to the left of the hopper two times (FR2), the house light extinguished, the panel lights above the lever changed from red to green and the pellet dispenser released one 45-mg chocolate pellet (BioServe, New Jersey) into the hopper. Presses on the right lever had no programmed consequences. Presses on the left lever were considered correct and presses on the right lever, incorrect. The number of lever presses, the number of times a rat poked its nose into the hopper and locomotor activity were recorded automatically (Graphic State Notation 2 Version 2.002-00, Coulbourn Instruments).

\subsubsection{Immunohistochemistry}

Sixty minutes after the end of the WM probe test (recent and remote), rats were placed into a Decapicone (Braintree) and decapitated. Brains were rapidly removed and hemisected. Right hemispheres were kept in $4 \%$ paraformaldehyde/0.01M phosphate buffer solution (PB; pH7.4) overnight at $4^{\circ} \mathrm{C}$. Brains were then cryoprotected in a $30 \%$ sucrose/0.01M PB solution the following day and stored at $4^{\circ} \mathrm{C}$ for at least 72 hours or until sectioned. Right hemispheres were sectioned at $25 \mu \mathrm{m}$ on a Leica CM1900 cryostat (Weztler, Germany). Sections were stored in a $0.1 \%$ sodium azide $/ 0.01 \mathrm{M} \mathrm{PB}$ solution at $4^{\circ} \mathrm{C}$.

Sections were washed for $3 \mathrm{X} 5$ minutes in a $0.2 \%$ Triton-X/0.01M phosphatebuffered saline (T-PBS) solution then blocked for 15 minutes in a $0.3 \% \mathrm{H}_{2} \mathrm{O}_{2}$ T-PBS solution. Sections were washed for $3 \mathrm{X} 5$ minutes in T-PBS solution then blocked in a $3 \%$ Animal Free Blocker (AFB) (Vector)/ T-PBS solution for 30 minutes followed by incubation in the primary antibody (rabbit anti-c-Fos from Abcam, 1:5000) overnight at room temperature. The following day, sections were washed for 3X10 minutes in T-PBS solution then incubated for two 
hours in the secondary antibody (biotinylated goat anti-rabbit, 1:500). Sections were then washed 3 X10 minutes in T-PBS followed by one-hour incubation in an avidin-biotinylated complex (ABC Elite kit; Vector Laboratories). Sections were given a wash in PBS then reacted with a $0.25 \%$ 3,3'-diaminobenzidine tetrahydrochloride (DAB) solution in PBS solution containing $0.0025 \% \mathrm{H}_{2} \mathrm{O}_{2}$ for 6 minutes. All sections were given a final rinse in 0.01M PBS for 15 minutes and mounted on glass slides. Sections were dehydrated and coverslipped with glass coverslips and Permount (Sigma).

\section{c-Fos Quantification}

Utilizing unbiased stereological principles with the Optical Fractionator method, an estimate of the number of c-Fos positive cells in the ACC and CA1 region of the dorsal hippocampus was undertaken. Stained sections were visualized using an Olympus BX51 brightfield microscope with a motorized stage (Olympus Canada, Markham, ON) and images captured with an Olympus U-CMAD3 camera. Stereo Investigator (MBF Bioscience, Williston, VT) software was used for unbiased stereological quantification. The ACC volume of interest was constricted in the anterior-posterior plan from bregma +1.2 to $+1.0 \mathrm{~mm}$. The medial border was the midline of the brain and the lateral border was the corpus callosum. The CA1 volume of interest focused on the anterior aspect of the dorsal hippocampus and was constricted in the anterior-posterior plan from bregma -3.4 to $-3.6 \mathrm{~mm}$. The region of interest (ACC or CA1 of the hippocampus) for each section was traced digitally at $4 \mathrm{X}$ magnification, with reference to Paxinos and Watson (2006) (see figure 2.5A and 2.7A). Two to three random coronal sections were sampled from each of these regions from each rat. c-Fos counting was performed using sampling parameters sufficient to produce a Gundersons coefficient of error $(\mathrm{GCE}, \mathrm{m}=1$ ) less than 0.1 , which has been established to be a suitable coefficient of error estimate (Blum et al., 2012; Gundersen and Jensen, 1987; Gundersen et al., 1999). A minimum of 4 animals per group was included in analysis. Not all animals could be included in analysis due to various factors such as missing sections for some animals. Counting parameters were set to a counting frame of $50 \times 50 \mu \mathrm{m}^{2}$ (ACC) and $30 \mathrm{X} 30 \mu \mathrm{m}^{2}$ (CA1) and a dissector height of $10 \mu \mathrm{m}$ between the top and bottom guard zones with average mounted section thickness of $16.0 \mu \mathrm{m}$ (ACC) and $14.9 \mu \mathrm{m}$ (CA1). c-Fos positive cells were quantified using a $60 \mathrm{X}$ magnification lens (oil immersion, NA 1.35) when the uppermost tip of c-Fos positive nuclei were in focus within the counting frame and the dissector height. Stereo Investigator software used planar and depth information for each counted nuclei to calculate the volume for the digitally traced region of interest. Quantification is represented as an estimated total per mean measured thickness per $10000 \mu \mathrm{m}^{3}$ to allow for comparisons across brain sections. 


\subsection{Results}

\subsubsection{Behavioural Training}

Figure 2.2 depicts performance of animals during behavioural training. All groups received WM training. Latency to reach the hidden platform was recorded (Figure 2.2A). A two-way, repeated measures ANOVA (group by day) revealed a main effect of day $\left(\mathrm{F}_{(4,176)}=132.9, p<0.001\right)$. No significant differences were observed between the different experimental groups.

Two groups received training on the WM followed by training on the RAM: one assigned to receive a recent $\mathrm{WM}$ probe test and one assigned to receive a remote WM probe test. The number of errors (unbaited arm entries and re-entries) made during training was analyzed with a two-way, repeated measures ANOVA (group by day; Figure 2.2B). A main effect of training day was found $\left(\mathrm{F}_{(3,42)}=23.7, p<0.001\right)$ demonstrating fewer errors made across days. No significant difference between the two groups was found. Within each group, a significant effect of day was found (WM/RAM:recent $\left(\mathrm{F}_{(3,21)}=5.7, p<0.01\right)$ WM/RAM:remote $\left(\mathrm{F}_{(3,21)}=2.7, p<0.05\right)$ indicating learning across days.

Two groups of animals received WM training followed by OP: one assigned to a recent WM probe test and one assigned to a remote WM probe test. The number of correct lever presses was analyzed with a two-way, repeated measures ANOVA (group by day; Figure 2.2C). A main effect of training day was found $\left(\mathrm{F}_{(4,64)}=27.7, p<0.001\right)$ demonstrating performance on the operant task improved across days. No significant group differences were observed.

\subsubsection{Water Maze Probe}

Rats received either a recent or remote WM probe test. Note that two rats did not have their probe performance tracked properly and were not included in the analyses. The amount of time spent swimming in the target quadrant (quadrant where platform was located during testing) and opposite quadrant, were examined (Figure 2.3A and 2.3B). Separate 3X2 (behaviour (WM, WM/RAM, or WM/OP) X time (recent or remote)) fixed factor ANOVAs were run. Statistical analyses on time spent in the target quadrant (Figure 2.3A) revealed a significant interaction between behaviour and time $\left(\mathrm{F}_{(2,41)}=5.7, p<0.01\right)$. Tukeys post hoc test revealed group WM/RAM:recent spent significantly more time swimming in the target quadrant than group WM/RAM:remote $(p<0.05)$. No other experimental groups differed significantly from one another.

Analysis of time spent in the opposite quadrant (Figure 2.3B) revealed a main effect of time $\left(\mathrm{F}_{(1,41)}=6.3, p<0.05\right)$ and a significant interaction between behaviour and time $\left(\mathrm{F}_{(2,41)}=3.6, p<0.05\right)$. Post hoc comparisons using Tukeys test revealed group WM/RAM:recent spent significantly less time swimming in the opposite quadrant than WM/RAM:remote $(p<0.05)$. No other experimental groups sharing behavioural procedures (i.e. WM:recent vs WM:remote or WM/OP:recent vs WM/OP:remote) differed significantly from one another.

Further analyses on the probe test examined the time spent in the target 
quadrant in 15 second slices (Figure 2.4A-D). Time spent in the target quadrant was analyzed from 0-15 seconds (Figure 2.4A), 15-30 seconds (Figure 2.4B), 3045 seconds (Figure 2.4C) and 45-60 seconds (Figure 2.4D). A repeated measures ANOVA (with time slice as the repeated factor) revealed a significant effect of time slice $\left(\mathrm{F}_{(3,123)}=13.47, p<0.01\right)$ and a significant 3 -way interaction between time slice, behaviour and time $\left(\mathrm{F}_{(6,123)}=2.29, p<0.05\right)$. Pairwise comparisons (using a Bonferroni correction) revealed the $0-15$ second time slice was significantly different from all other time slices $(p<0.01)$. Based on these findings, individual ANOVAs were run at each time slice. Rats were expected to spend $25 \%$ of their time in any of the four quadrants due to chance. One sample ttests were run on experimental groups across the different time slices to examine group preferences. From 0-15 seconds (Figure 2.4A), all experimental groups, except WM/RAM:remote, spent above chance amounts of time in the target quadrant $(p<0.05)$. Only WM/RAM:recent group spent above chance amounts of time in the target quadrant for all of the time slices.

\subsection{3 c-Fos expression in the $\mathrm{ACC}$}

Figure 2.5A depicts the ACC region where c-Fos was counted and Figure 2.5B displays representative images at $4 \mathrm{X}$ and $60 \mathrm{X}$ magnification. Figure 2.5C shows representative images of c-Fos staining in the ACC at 20X magnification to depict group differences. Figure 2.6 shows the quantification of the number of c-Fos positive nuclei per $10000 \mu \mathrm{m}^{3}$ in the ACC. A 3X2 fixed factor ANOVA revealed a main effect of behaviour $\left(\mathrm{F}_{(2,25)}=4.0, p<0.05\right)$, a main effect of time $\left(\mathrm{F}_{(1,25)}=\right.$ $31.4, p<0.01)$, and a significant interaction of behaviour $\mathrm{X}$ time $\left(\mathrm{F}_{(2,25)}=5.6\right.$, $p<0.05)$. Further analyses revealed a main effect of group $\left(\mathrm{F}_{(5,25)}=9.0, p<0.01\right)$. Tukeys post hoc test revealed group WM:recent had a significantly lower number of c-Fos positive nuclei per $10000 \mu \mathrm{m}^{3}$ in the ACC compared to all remotely tested groups (WM:remote, WM/RAM:remote, WM/OP:remote) as well as compared to WM/RAM:recent $(p<0.05)$. Similarily, group WM/OP:recent had a significantly lower number of c-Fos positive nuclei per $10000 \mu \mathrm{m}^{3}$ in the

ACC compared to all remotely tested groups (WM:remote, WM/RAM:remote, WM/OP:remote) $(p<0.05)$ as well as approached significance compared to group WM/RAM:recent $(p=0.07)$. Group WM/RAM:recent was not significantly different from any of the remotely tested groups (WM:remote, WM/RAM:remote, WM/OP:remote). All remotely tested groups had a significantly higher number of c-Fos positive nuclei per $10000 \mu \mathrm{m}^{3}$ in the ACC compared to WM:recent and WM/OP:recent $(p<0.05)$, but not compared to the WM/RAM:recent group.

\subsection{4 c-Fos expression in the CA1 of the hippocampus}

Figure 2.7A depicts the CA1 region of the hippocampus where c-Fos was counted and Figure 2.7B displays representative images at $4 \mathrm{X}$ and $60 \mathrm{X}$ magnification. Figure $2.7 \mathrm{C}$ shows representative images of c-Fos staining in the CA1 of the hippocampus at 20X magnification to depict group differences. Figure 2.8 shows the quantification of the number of c-Fos positive nuclei per $10000 \mu \mathrm{m}^{3}$ in the 
CA1. A 3X2 fixed factor ANOVA revealed a main effect of time $\left(\mathrm{F}_{(1,28)}=14.5\right.$, $p<0.01)$ with recently tested animals revealing a significantly lower number of c-Fos positive nuclei per $10000 \mu \mathrm{m}^{3}$ in the CA1 compared to remotely tested groups.

\subsection{Discussion}

Behavioural findings indicated rats that were trained on two hippocampaldependent tasks and tested recently (WM/RAM:recent) outperformed all other behavioural groups on the water maze probe test. Additionally, the WM/RAM: recent group displayed a pattern of c-Fos staining in the anterior cingulate cortex similar to that seen in remotely-tested groups and different from that seen in the other recently-tested groups, suggesting multiple hippocampal-dependent memories may accelerate the speed at which memories show reliance on structures in addition to the hippocampus.

Although not exclusively (Moscovitch et al., 2006; Rosenbaum et al., 2008; Winocur et al., 2010), a great deal of human research supports the idea of a time limited role for the hippocampus in memory storage (Bayley et al., 2006; Kapur and Brooks, 1999; Manns et al., 2003; Rosenbaum et al., 2000; Teng and Squire, 1999). This is evidenced by damage to the hippocampus resulting in temporally graded retrograde amnesia, whereby memories from the recent past are lost but memories from the remote past are spared. An evident inconsistency exists within animal research, where hippocampal damage sometimes results in temporally graded retrograde amnesia (Anagnostaras et al., 1999; Kim and Fanselow, 1992; Kubie et al., 1999; Maviel et al., 2004; Teixeira et al., 2006; Zola-Morgan and Squire, 1990) and sometimes results in a flat or ungraded retrograde amnesia (Broadbent et al., 2006; Clark et al., 2005a,b, 2007; Epp et al., 2008; Lehmann et al., 2007, 2013; Mumby et al., 1999; Sparks et al., 2011; Sutherland et al., 2008, 2001). This inconsistency becomes complicated to examine because behavioural measures used to assess memory in animals makes it difficult to determine whether the hippocampus is required for performance of the task being used for assessment or whether the hippocampus is required for a memory storage/ retrieval process. It is possible that the number of memories processed over the lifetime underlies this discrepancy. In humans, there is a seemingly endless sequence of memory storage and retrieval, while laboratory animals are typically required to retrieve and store only one memory for one behavioural task. In the absence of multiple memories competing for processing space in the hippocampus, the consolidation process may not be as quick to progress and a hippocampal-dependent memory may not be as likely to require, and thus form, extra-hippocampal (cortical) connections, thereby relying heavily on hippocampal involvement for an extended period of time. We propose that several consecutive hippocampal-dependent memories (resulting from multiple hippocampal-dependent tasks) may compete for hippocampal processing space, resulting in an accelerated reliance on cortical structures.

The WM is the classic test of spatial function in rodents. Bilateral lesions 
or inactivation of the hippocampus and associated areas (dentate gyrus, subiculum, entorhinal cortex) impair performance on this task (Broadbent et al., 2006; Clark et al., 2005a,b; D'Hooge and De Deyn, 2001; Holahan and Routtenberg, 2011; Morris et al., 1986, 1982; Ramos, 2009; Remondes and Schuman, 2004; Sutherland et al., 2001, 1983; Teixeira et al., 2006; Wiltgen et al., 2010). The RAM is a spatial task considered to be hippocampal-dependent (Bouffard and Jarrard, 1988; Jarrard, 1978; Olton and Samuelson, 1976). Lesion studies demonstrate the importance of a functioning hippocampus in the RAM task (Bouffard and Jarrard, 1988; Jarrard, 1976, 1978; Olton and Papas, 1979; Olton et al., 1978). The operant conditioning task used in this study is typically not dependent on the hippocampus (Carvalho et al., 2001; Corbit and Balleine, 2000; Gallagher and Hollandt, 1992; Shull and Holloway, 1985). As both the WM and RAM tasks are dependent upon the hippocampus, training on both of these tasks may result in the activation of similar neural pathways within the hippocampus and result in an accelerated progression of the consolidation process, whereby memories which were initially heavily dependent upon circuits within the hippocampus show increasing reliance upon cortical regions.

Behavioural analyses on the water maze probe test revealed group WM/RAM: recent spent significantly more time swimming in the target quadrant and less time swimming in the opposite quadrant than their remote counterparts. Time slice data revealed WM/RAM:recent group as the only experimental group that spent significantly higher than chance amounts of time swimming in the target quadrant throughout all of the time slices. The sustained preference for the target quadrant in group WM/RAM:recent indicates a strong memory for the platform location. Curiously, the enhanced behavioural performance in the WM/RAM:recent group during the WM probe test was not seen in their remote counterparts. The WM/RAM:remote group did not differ significantly from any of the other groups in terms of preference for the target or opposite quadrant but did perform noticeably worse than their recent counterparts. However, we dont believe our findings convincingly demonstrate an impairment in the WM/RAM:remote group. Although the WM/RAM:remote group did not spend above chance amounts of time in the target quadrant during the first time slice (when all other groups did), their mean time spent in the target quadrant did not differ significantly from other behavioural groups. Further experiments investigating hippocampus and ACC protein expression, morphological changes, and behavioural findings from ACC inactivation are planned to examine this finding.

To complement the behavioural findings, it was of interest to examine c-Fos expression in the ACC, an area implicated in remote memory storage (Bontempi et al., 1999; Frankland et al., 2004; Holahan et al., 2007; Lopez et al., 2012; Maviel et al., 2004; Restivo et al., 2009; Teixeira et al., 2006; Vetere et al., 2011; Weible et al., 2012). The pattern of c-Fos expression observed in the group trained on two hippocampal-dependent tasks and tested recently (WM/RAM:recent), the same group which showed enhanced behavioural performance, showed a similar c-Fos pattern of expression as seen in groups probed remotely. The WM/RAM:recent group showed significantly higher levels of c-Fos 
expression than the other recently probed groups. This increase in c-Fos expression in the ACC is not suspected to be due to increased behavioural training or stimulation, as recently probed animals trained on two tasks (one hippocampaldependent (WM) and one non-hippocampal-dependent (OP)) did not show an increase in c-Fos expression in the ACC compared to recently probed animals trained on only one hippocampal-dependent task (WM). Therefore, this increase in c-Fos staining in the ACC seems to be a unique change conferred to this specific behavioural group. Thus, it appears recruitment of the ACC may not only be time-dependent, but hippocampal demand-dependent. The increase in c-Fos positive nuclei in all behavioural groups at the remote time point supports previous findings of a remote time-dependent role of the ACC, but the increase in c-Fos positive nuclei in the WM/RAM:recent group also argues the ACC may be recruited in times of hippocampal demand.

c-Fos expression in the CA1 of the hippocampus was examined as well. Stereological analyses revealed remotely tested groups had significantly higher numbers of c-Fos positive nuclei than recently tested groups. This suggests the hippocampus is still very much involved in retrieval (and perhaps storage) of the memory for the water maze task even at a remote time point.

The behavioural and activational results presented here suggest training on sequential hippocampal-dependent tasks accelerates the rate at which hippocampaldependent memories show an additional reliance on cortical structures. The seemingly early activation observed in the ACC of rats trained on two hippocampaldependent tasks may be indicative of an accelerated recruitment of cortical structures in the retrieval of recently stored memories. It should also be considered that this early reorganization of neuronal structures may underlie the enhanced behavioural performance seen in this group of animals. 


\section{Supplemental material not included in Manuscript 1}

\subsection{Supplemental Introduction}

To provide a more complete activation profile associated with the retrieval of memories following training on the multiple memory behavioural procedure, we examined protein labeling patterns of activity-regulated cytoskeleton-associated protein/ activity-regulated gene 3.1 (Arc/Arg 3.1). Arc is an immediate early gene protein product of interest in memory consolidation. Arc mRNA is localized selectively to dendrites that have recently been activated possibly labeling potentiated synapses (Kovács, 2008; Kuhl and Skehel, 1998; Link et al., 1995; Lyford et al., 1995; Steward et al., 1998; Steward and Worley, 2001).

Arc protein labeling is associated with memory consolidation, but not acquisition (Guzowski et al., 2000). Guzowski and colleagues infused Arc antisense oligodeoxynucleotides (ODNs) into the hippocampus prior to spatial water maze training. Controls and Arc antisense rats performed similarly during acquisition, but Arc antisense rats exhibited a deficit in retention when tested 48 hours later. Plath et al. (2006) showed Arc knockout mice exhibited deficiencies in LTP2/3 and the formation of long-lasting memories, while LTP1 and short-term memories were not affected. Behavioural tasks used by Plath et al. (2006) included hippocampal-dependent and independent tasks. Arc knockout mice demonstrated impaired spatial learning strategies when tested on the water maze task. They spent significantly less time in the target quadrant compared to wildtype controls and had significantly fewer annulus crossings over the trained platform location. In addition, Arc knockout mice were slower to learn a new platform location. The deficits exhibited on the water maze task were assumed to result from impairments in cognitive function, as sensory-motor functions were intact demonstrated by normal swim velocities, time floating and performance on a visible platform task. Both wildtypes and Arc knockouts showed a short-lasting fear association to a context and tone paired with shock during acquisition but knockout mice showed significantly lower levels of freezing to both the context and tone compared to wildtype controls 24 hours later. In addition, both wildtypes and Arc knockouts displayed short-term object-recognition memory but, when tested 24 hours later, the Arc knockout mice showed a lack of object-recognition memory. Finally, Arc knockout mice did not form a longterm conditioned taste aversion memory despite normal gustatory processing.

Arc mRNA levels are low at baseline but become upregulated in the presence of stimulation which results in LTP2/3 (Guzowski et al., 2000; Link et al., 1995; Lyford et al., 1995). Guzowski et al. (2000) used intrahippocampal infusions of Arc antisense ODNs to inhibit Arc protein expression and determine the effect on LTP. Initial LTP amplitude did not differ significantly between rats treated with antisense ODNs and scrambled ODNs, suggesting LTP induction was not affected by the inhibition of Arc protein expression. The rate of decay of LTP in Arc antisense treated rats was much more rapid than that seen in Arc scrambled rats, suggesting Arc protein expression plays a role in the maintenance of 
LTP. Messaoudi et al. (2007) injected Arc antisense two hours after the onset of high frequency stimulation (HFS) and found a rapid reversal of LTP with field excitatory postsynaptic potential recordings returning to baseline levels within 60 minutes. Interestingly, no effect was seen when Arc antisense was injected four hours following HFS, indicating a time sensitive role of Arc in LTP maintenance.

Arc, an immediate early gene, is not a transcription factor, but a cytosolic protein downstream from many neuronal signaling pathways (Shepherd and Bear, 2011). It is clear that Arc plays a role in memory consolidation, but it is unclear how Arc functions to stabilize memory. Shepherd et al. (2006) found that high levels of Arc result in increased AMPAR endocytosis, while low levels of Arc result in increased surface expression of AMPAR and low levels of endocytosis. Bramham et al. (2010) suggested Arc may play a part in stabilizing structural changes at potentiated synapses as part of its role as a cytoskeletonassociated protein (see also Messaoudi et al. (2007)). Whatever the critical role(s) of Arc in memory stabilization are, it seems clear that proper regulation of Arc expression levels is crucial.

In addition to its local synthesis at dendrites, Arc undergoes nonsensemediated decay, which restricts the translation of an mRNA to protein (Giorgi et al., 2007; Shepherd and Bear, 2011). Arc expression follows a distinct temporal course. Following a stimulus, Arc RNA is detected in neuronal nuclei within two minutes (Guzowski et al., 2005). Arc mRNA is detected in cytoplasm and dendrites 20-60 minutes following the stimuli. Recently, it has been suggested that Arc protein produced in dendrites may be transported back to the nucleus, bringing with it signaling molecules in a signaling endosomal compartment (Shepherd and Bear, 2011). The precise function of dendritic compared to somatic Arc protein expression remains unknown.

The present study used immunohistochemistry to examine Arc protein expression in rats that underwent training on the multiple memory behavioural procedure to determine regional differences in Arc distribution at recent and remote time points.

\subsection{Supplemental Materials \& Methods}

Subjects, behavioural procedures and tissue processing were as described for Manuscript 1. Tissue slices were processed using an anti-Arc antibody. A minimum of 4 sections per group were included in analyses.

\subsubsection{Immunohistochemistry}

Sections were washed for $3 \mathrm{X} 5$ minutes in a $0.2 \%$ Triton-X/0.01M phosphatebuffered saline (T-PBS) solution then blocked for 15 minutes in a $3 \% \mathrm{H}_{2} \mathrm{O}_{2}$ T-PBS solution. Sections were washed for $3 \mathrm{X} 5$ minutes in T-PBS solution then blocked in a $5 \%$ Animal Free Blocker (2.5X concentrated) (AFB) (Vector)/ TPBS solution for 30 minutes followed by incubation in the primary antibody 
(rabbit anti-Arc from Synaptic Systems, 1:500) overnight at room temperature. The following day, sections were washed for 3 X10 minutes in T-PBS solution then incubated for two hours in the secondary antibody (biotinylated donkey anti-rabbit, 1:500). Sections were then washed 3X10 minutes in T-PBS followed by one-hour incubation in an avidin-biotinylated complex (ABC Elite kit; Vector Laboratories). Sections were given a wash in PBS then reacted with a $0.25 \%$ 3,3'-diaminobenzidine tetrahydrochloride (DAB) solution in PBS solution containing $0.0025 \% \mathrm{H}_{2} \mathrm{O}_{2}$ for 6 minutes. All sections were given a final rinse in $0.01 \mathrm{M}$ PBS for 15 minutes and mounted on glass slides. Sections were dehydrated and coverslipped with glass coverslips and Permount (Sigma).

\subsubsection{Arc Quantification}

Stained sections were visualized using an Olympus BX51 brightfield microscope with a motorized stage (Olympus Canada, Markham, ON) and images captured with an Olympus U-CMAD3 camera. For each stained section, images of the ACC, CA1 and CA3 were captured at 20X magnification. When the entire region of interest could not be captured in a single image, a sample of the area was acquired.

Image J software (NIH) was used to aid in Arc quantification. The entire ACC was captured at 20X magnification resulting in an image of $1600 \mathrm{X} 1200$ pixels. For the CA1 and CA3, a rectangular box $1600 \mathrm{X} 600$ pixels was used to estimate Arc staining levels. Images were processed to 8-bit type, which converted them to gray scale with 256 shades of gray. Images were then converted to binary by adjusting the threshold such that gray scale values below a cutoff point became black and those above the cutoff became white. Particles between 50-750 pixels in size were analyzed. Circularity was defined differently for cell body and dendritic staining. Circularity is defined as $4 \pi\left(\right.$ area/perimeter $\left.{ }^{2}\right)$ and can range from 0 (infinitely elongated polygon) to 1 (perfect circle). To examine cell body (somatic) Arc staining, circularity was set between 0.7-1.0. To examine dendritic Arc staining, circularity was set between 0-0.4. To ensure the appropriateness of the counts, outlines of the particles were displayed. Counts were made by an experimenter blinded to behavioural manipulations. A twoway ANOVA with behavioural manipulation (WM Only, WM/RAM, WM/OP) and time (recent or remote) as the fixed factors and somatic or dendritic Arc staining counts as the dependent variable were carried out in each of the areas of interest.

\subsection{Supplemental Results}

\subsubsection{ACC}

Figure 2.9A depicts representative Arc staining in the ACC. Figures $2.9 \mathrm{~B}$ and $2.9 \mathrm{C}$ show quantification of Arc staining in the soma and dendrites, respectively. Statistical analyses for somatic staining revealed a main effect of time 
$\left(\mathrm{F}_{(1,18)}=5.38, p<0.05\right)$, with recent groups (133) expressing more somatic staining than remote groups (92). LSD post-hoc comparisons revealed group WM Only:Recent had more somatic staining compared to groups WM/RAM:Remote and WM/OP:Remote $(p \leq 0.05)$. Statistical analyses for dendritic staining revealed no main effect of behaviour $\left(\mathrm{F}_{(2,18)}=0.17, p<0.05\right)$, time $\left(\mathrm{F}_{(1,18)}=1.18\right.$, $p>0.05)$ and no significant interaction between the two $\left(\mathrm{F}_{(2,18)}=1.43, p>0.05\right)$.

\subsubsection{CA1}

Figure 2.10A depicts representative Arc staining in the CA1. Figures 2.10B and $2.10 \mathrm{C}$ show quantification of Arc staining in the soma and dendrites, respectively. Statistical analyses for somatic staining revealed no main effect of behaviour $\left(\mathrm{F}_{(2,24)}=1.55, p<0.05\right)$, time $\left(\mathrm{F}_{(1,24)}=0.54, p<0.05\right)$, or significant interaction between the two $\left(\mathrm{F}_{(2,24)}=0.85, p<0.05\right)$. Statistical analyses for dendritic staining revealed no main effect of behaviour $\left(\mathrm{F}_{(2,24)}=3.14, p<0.05\right)$, time $\left(\mathrm{F}_{(1,24)}=0.19, p<0.05\right)$, or significant interaction between the two $\left(\mathrm{F}_{(2,24)}=0.43\right.$, $p<0.05)$. Planned post-hoc comparisons revealed greater dendritic staining in group WM/RAM:Recent (109) compared to group WM Only:Recent (57) $(p<0.05)$.

\subsubsection{CA3}

Figure 2.11A depicts representative Arc staining in the CA3. Figures 2.11B and $2.11 \mathrm{C}$ show quantification of Arc staining in the soma and dendrites, respectively. Statistical analyses for somatic staining revealed no main effect of behaviour $\left(\mathrm{F}_{(2,19)}=0.06, p>0.05\right)$, time $\left(\mathrm{F}_{(1,19)}=1.58, p>0.05\right)$, or significant interaction between the two $\left(\mathrm{F}_{(2,19)}=0.11, p>0.05\right)$. Statistical analyses for dendritic staining revealed no main effect of behaviour $\left(\mathrm{F}_{(2,19)}=1.15, p>0.05\right)$, time $\left(\mathrm{F}_{(1,19)}=1.81, p>0.05\right)$, or significant interaction between the two $\left(\mathrm{F}_{(2,19)}=0.08\right.$, $p>0.05)$.

\subsection{Supplemental Discussion}

Arc is well-conserved across vertebrates and is induced by various behavioural procedures in many species (Bramham et al., 2008). Because Arc mRNA is rapidly transported to dendrites that have recently been activated and can undergo local synaptic transcription (Moga et al., 2004; Rodríguez et al., 2005; Steward et al., 1998; Yin et al., 2002), we examined both somatic and dendritic Arc staining patterns in the ACC and CA1/CA3 regions after recent and remote memory retrieval. We have not come across this distinction (somatic versus dendritic) in other published papers using immunohistochemistry (Holloway and McIntyre, 2011; Thomsen et al., 2008; Wall et al., 2012; Zhang et al., 2011). It was of interest to study Arc labeling in regards to the multiple memory procedure, as neurons that are activated during memory recall might highlight potential sites for memory storage. 
A study by Gusev et al. (2005), examined Arc mRNA expression in the hippocampus following recent (24 hours) and remote (1 month) recall of a WM task. They found an overall decrease in hippocampal Arc activity in remote compared to recent recall. The present study did not reveal any significant differences in Arc somatic staining expression between recent and remote time points in the CA1 or CA3 of the hippocampus. Previous reports showed increased Arc mRNA labeling patterns in the ACC at recent compared to remote time points following a WM recall task (Gusev and Gubin, 2010). In agreement, the present study showed increased ACC somatic staining in recent, compared to remote, groups (Figure 2.9B).

Evidence supports a role of Arc during memory consolidation, but not memory retrieval. For example, Holloway and McIntyre (2011) reported evidence refuting an association between elevated Arc protein levels and memory retrieval. Rats were trained on an inhibitory avoidance task and administered a post-training intra-ACC infusion of Arc antisense ODNs to suppress Arc translation. Infusion of the ODNs 3 hours prior to the 48 hour retention test did not result in a performance deficit on the inhibitory avoidance task compared to control rats. Infusion of the ODNs 6 hours following initial training resulted in performance deficits during the 48 hour retention test. Montag-Sallaz and Montag (2003) examined Arc expression using in situ hybridization in mice and a one-trial learning paradigm where mice learned to escape a brightly lit compartment by entering a dark compartment. The control group had no access to the dark compartment, the learning group had access to the dark compartment for one trial, and the retrieval group had access to the dark compartment for two trials on consecutive days. Mice were sacrificed $15 \mathrm{~min}, 1$ hour, 4.5 hours or 6 hours following access to the dark compartment. Increased expression of Arc was shown in many brain areas including the ACC and CA1 in the learning group, but not in the control group or the retrieval group. The specific increase in Arc expression seen in the learning, but not retrieval group, provides support for a role for Arc in memory consolidation, but not retrieval.

Arc dendritic staining in the ACC and CA3 did not show any trends in expression levels, and no group differences were noted. Following the recent probe, group WM/RAM had more Arc dendritic staining in the CA1 compared to group WM Only. This suggests greater Arc activity in the CA1 of groups trained on two hippocampal-dependent tasks and tested recently. An increased demand placed upon the CA1 of the hippocampus during performance on the WM probe test may account for this increased activity. Findings from Gilbert et al. (2001) implicate the CA1 subregion of the hippocampus in temporal separation tasks. These researchers lesioned the CA1 of the hippocampus and found it to be important in separating temporal events. Rats trained on two hippocampal-dependent tasks were subjected to two spatial training events, the WM and the RAM. The CA1 is important in separating events across time, thus, the activity of the CA1 may be heightened following the WM probe test as RAM training had occurred for 5 days prior to this. Rats in this group may require more input from the CA1 (and so greater activation) in separating the spatial maps formed during these two tasks. 
Given the time line of the current behavioural procedure, it is likely we investigated a time point of Arc expression not often examined. Gusev and Gubin (2010) and Gusev et al. (2005) had similar time lines with a 9 day water maze training procedure, followed by a recent and remote probe test 24 hours or 1 month following the last training session, respectively. As noted, the present results supported the findings of these past manuscripts. Considering the suggestion that Arc protein produced in dendrites may transport signaling molecules back to the nucleus, it is possible the increased somatic Arc expression noted in the ACC at the recent time point indicated a process involved in systems consolidation. The increased dendritic Arc expression noted in group WM/RAM compared to WM Only could reflect increased consolidation for detailed spatial temporal information (detailed information is hypothesized by some to always be reliant upon the hippocampus (Nadel and Moscovitch, 1997). 


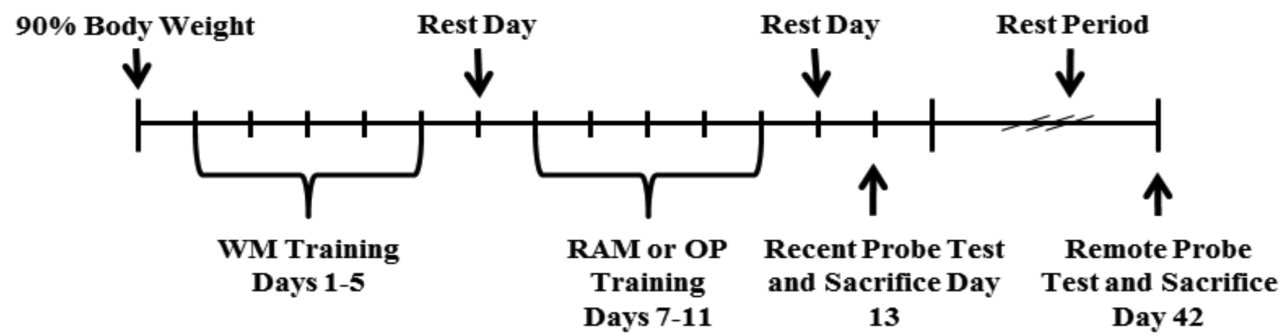

Figure 2.1: Timeline of experimental procedures. 

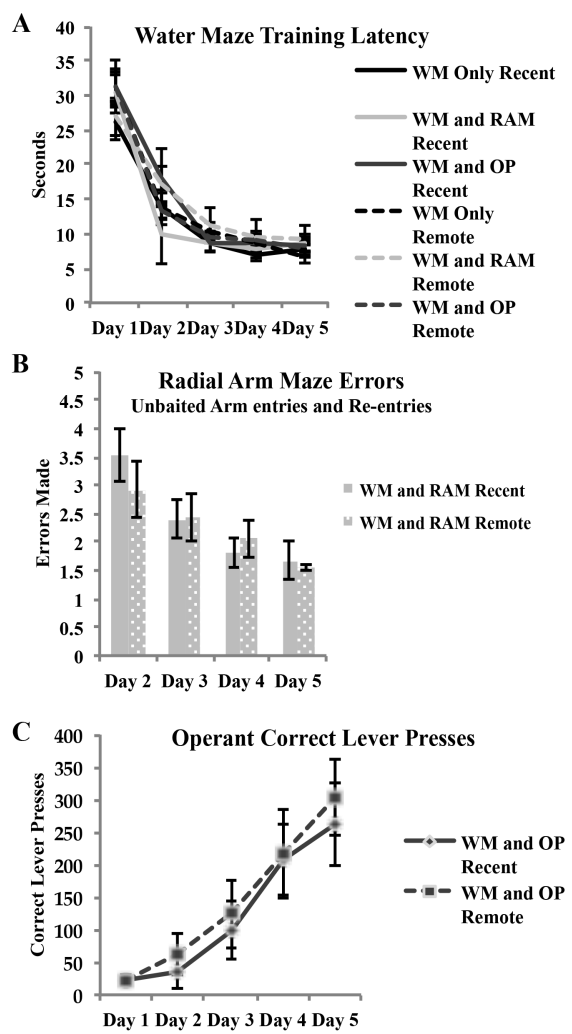

Figure 2.2: Behavioural training data. A) Average latencies to reach the hidden platform in the water maze task during the five days of training. No significant differences were seen between animals assigned to different behavioural groups. A main effect of day was found, showing a decrease in latency to reach the hidden platform across days, indicating an improvement in performance across training days. B) Average errors (unbaited arm entries and re-entries) made in the radial arm maze task during the five days of training. No significant differences were found in animals assigned to receive either a recent or remote water maze probe. A main effect of day was found within each group, showing a decrease in errors made across days, indicating an improvement in performance on the task across days. C) Average correct lever presses on the operant conditioning task. No significant differences were found in animals assigned to receive either a recent or remote water maze probe. A main effect of day was found, showing an increased number of lever presses made across days, indicating an improvement in performance on the task across days. 

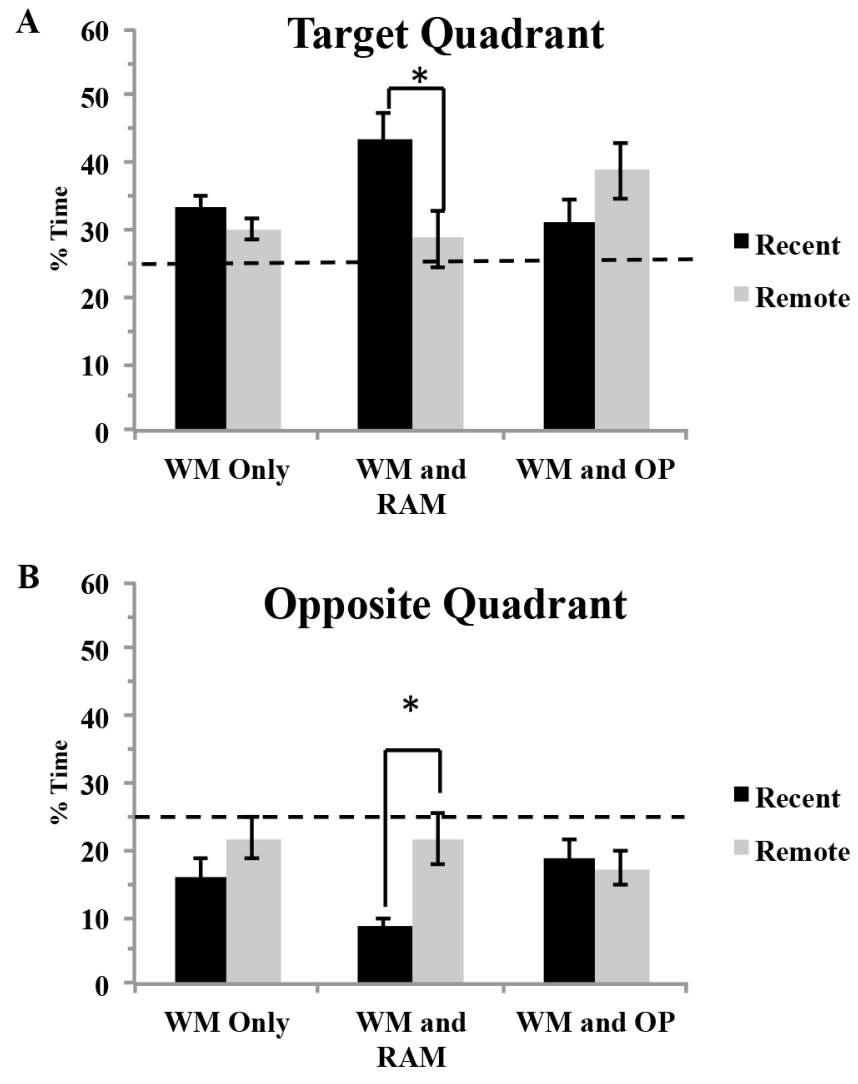

Figure 2.3: Percent time spent swimming in the target (A) and opposite (B) quadrant during the 60 second water maze probe. The dashed line represents chance performance (25\%). A) Animals trained on two hippocampal-dependent tasks and tested recently (WM/RAM:recent) spent significantly more time swimming in the target quadrant compared to their remotely probed counterparts (WM/RAM:remote). B) Animals trained on two hippocampal-dependent tasks and tested recently (WM/RAM:recent) spent significantly less time swimming in the opposite quadrant compared to their remotely probed counterparts (WM/RAM:remote). 

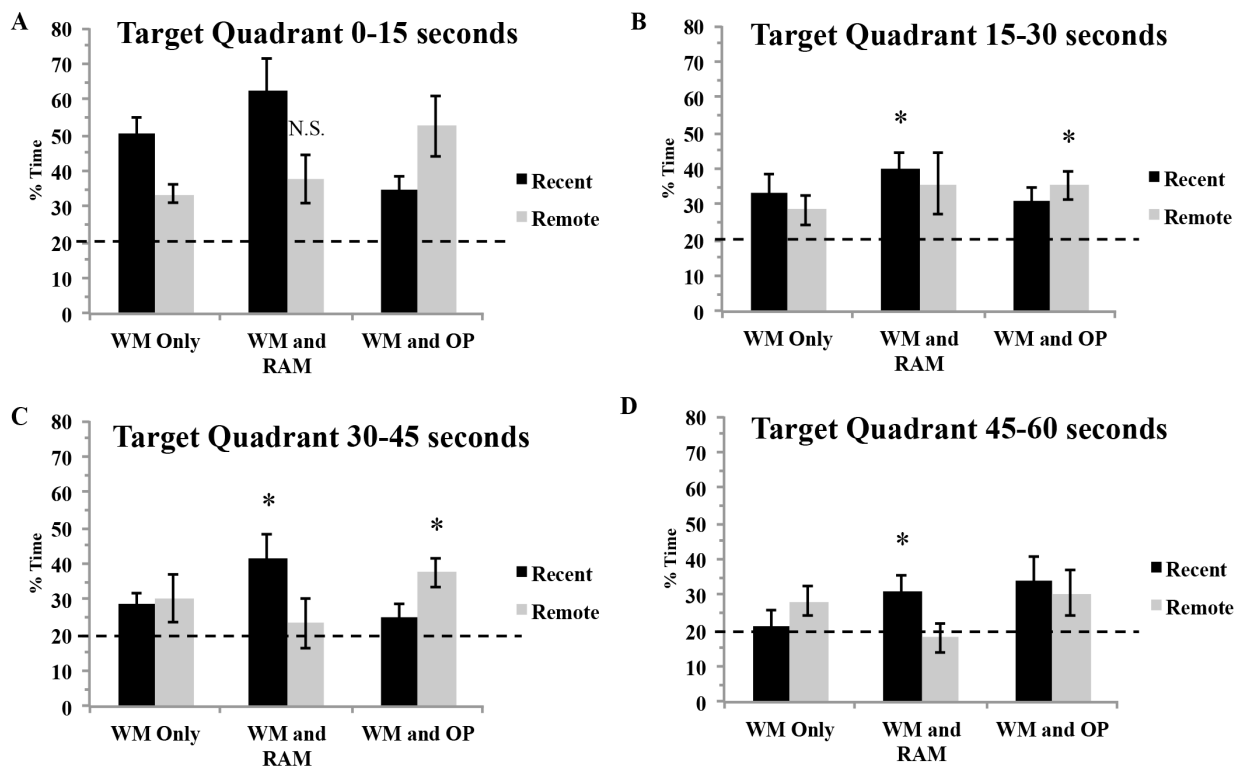

Figure 2.4: Percent time spent swimming in the target quadrant during the water maze probe in 15 second time slices. The dashed line represents chance performance (25\%). A) Percent time spent swimming in the target quadrant during the first 15 seconds of the water maze probe. All groups except for WM/RAM:remote group spent significantly higher than chance amounts of time in the target quadrant. B) Percent time spent swimming in the target quadrant during the 15-30 second time slice of the 60 second probe. C) Percent time spent swimming in the target quadrant during the 30-45 second time slice of the 60 second probe. D) Percent time spent swimming in the target quadrant during the 45-60 second time slice of the 60 second probe. The WM/RAM:recent group was the only group that spent significantly higher than chance amounts of time in the target quadrant. 
A

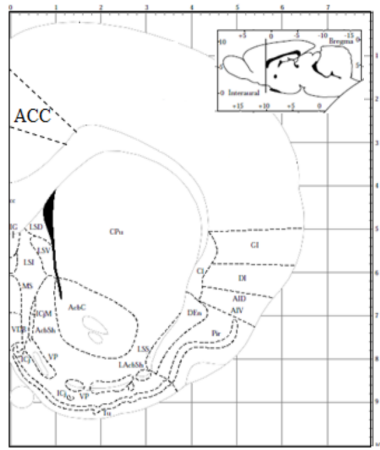

B

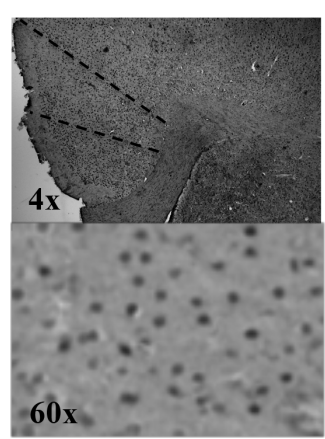

WM/OP

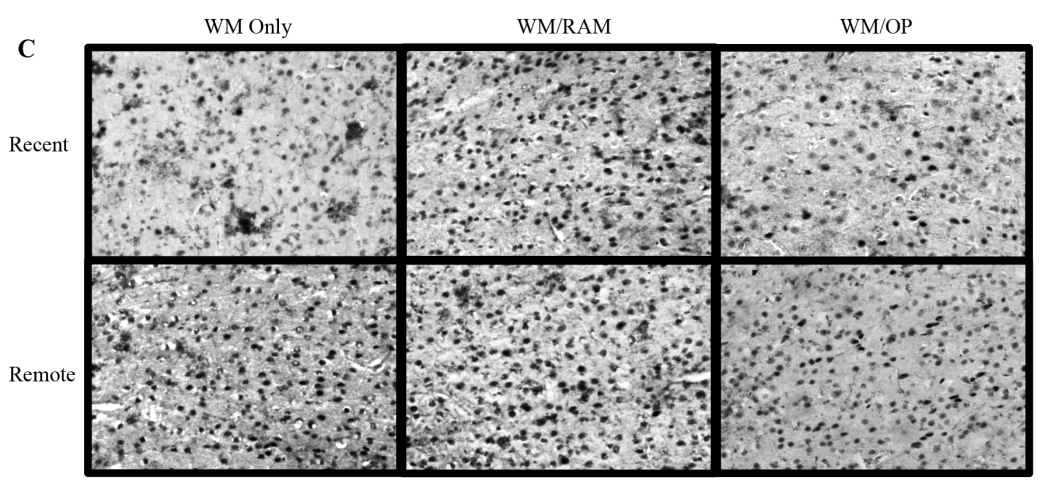

Figure 2.5: Representative images of c-Fos positive immunoreactive cells in the ACC A) Depiction of the ACC where c-Fos immunoreactive cells were counted (Paxinos and Watson, 2006). B) Images of the ACC at $4 \mathrm{X}$ and $60 \mathrm{X}$ magnification. C) Representative images of the ACC at 20X magnification to show group differences. 


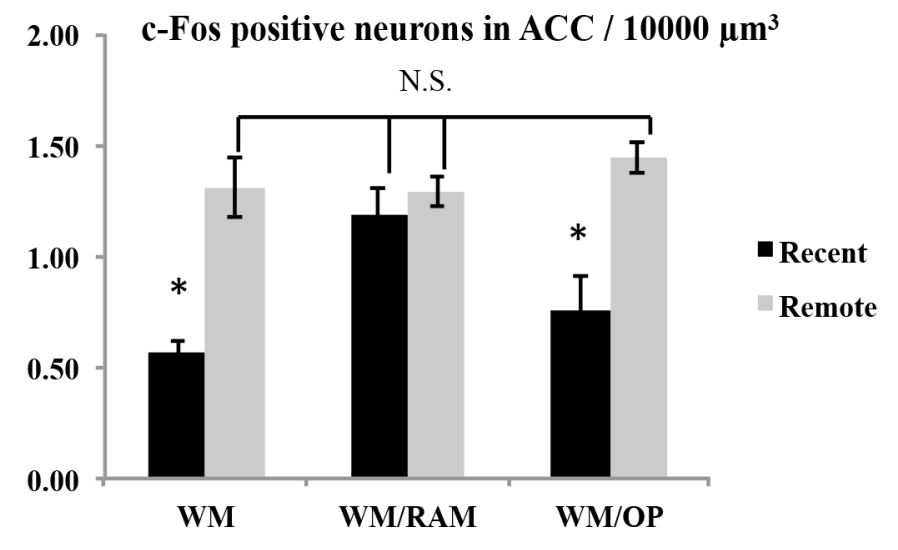

Figure 2.6: Stereological quantification of the number of c-Fos positive nuclei per $10000 \mu \mathrm{m}^{3}$ in the ACC. WM/RAM:recent group displays a pattern of c-Fos staining similar to the pattern staining observed in remotely tested groups. 
A

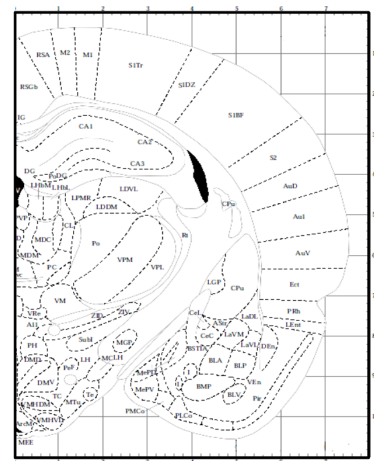

WM Only

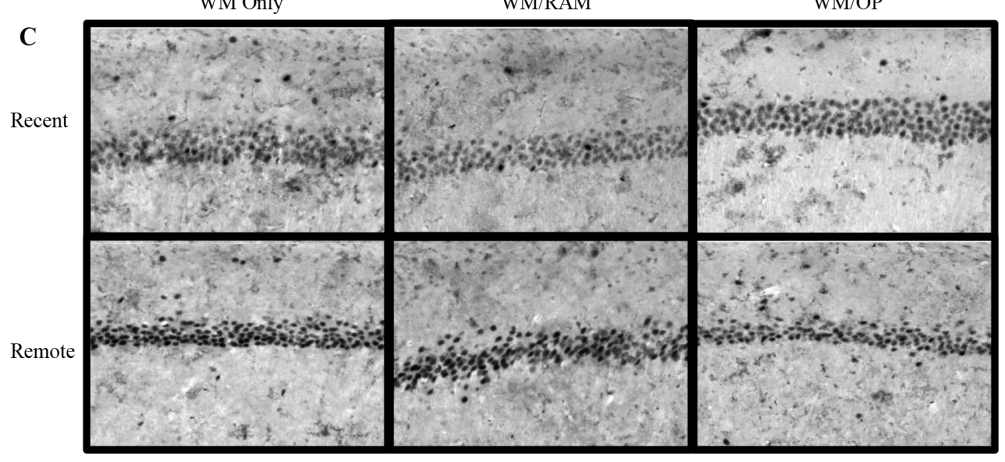

B
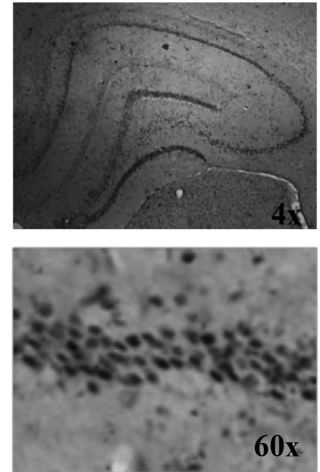

WM/OP

Figure 2.7: Representative images of c-Fos positive immunoreactive cells in the CA1 of the hippocampus A) Depiction of the CA1 where c-Fos immunoreactive cells were counted (Paxinos and Watson, 2006) B) Images of the CA1 at 4X and 60X magnification. C) Representative images of the CA1 at 20X magnification to show group differences. 


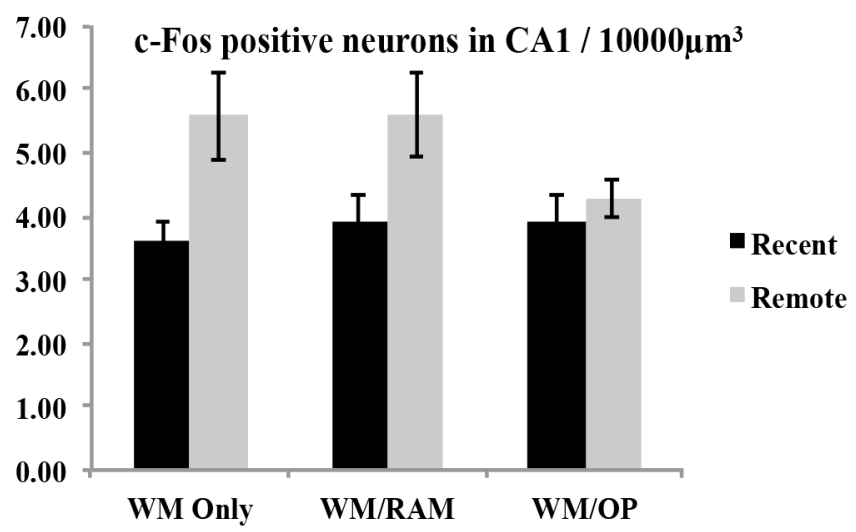

Figure 2.8: Stereological quantification of the number of c-Fos positive nuclei per $10000 \mu \mathrm{m}^{3}$ in the CA1. A main effect of time was found, with recently tested animals revealing significantly lower numbers of c-Fos positive nuclei compared to remotely tested groups. 
A

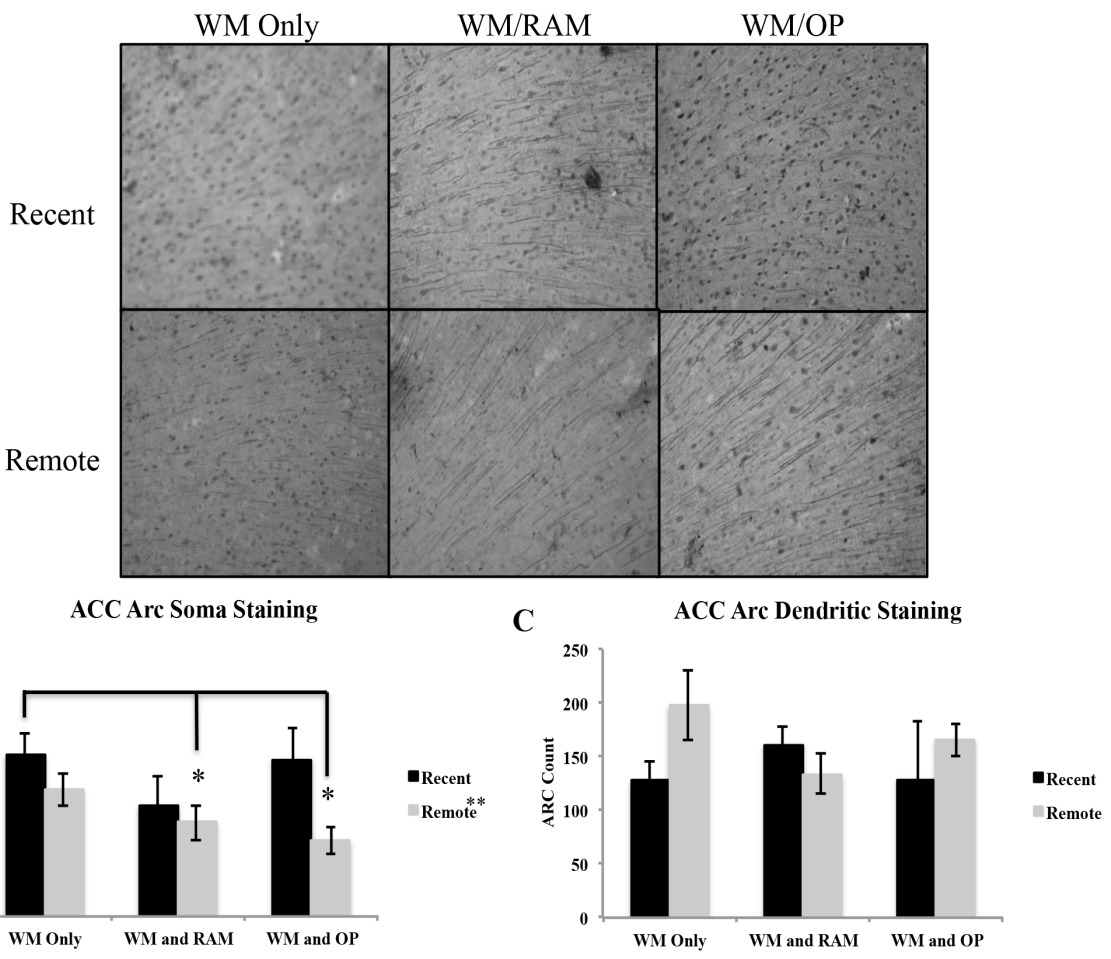

Figure 2.9: A) Representative images of Arc staining in the ACC at 20X magnification. B) Quantification of somatic Arc staining in the ACC. A main effect of time revealed recently probed groups expressed more somatic Arc staining compared to remotely probed groups. C) Quantification of dendritic Arc staining in the ACC revealed no significant differences between groups. 
A

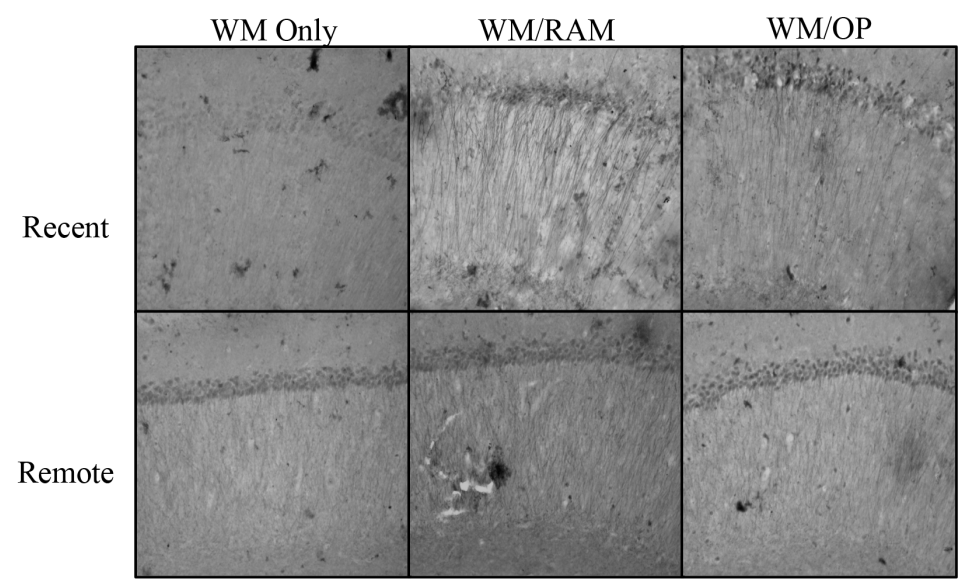

B

CA1 Arc Soma Staining

C

CA1 Arc Dendritic Staining
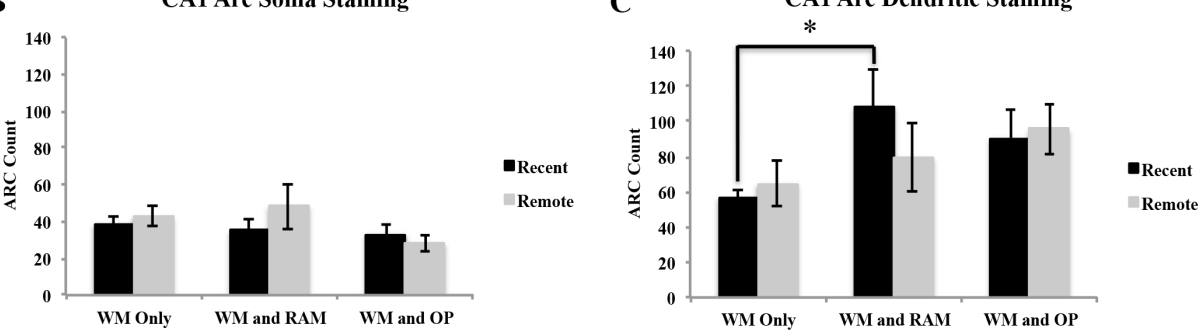

Figure 2.10: A) Representative images of Arc staining in the CA1 at 20X magnification. B) Quantification of somatic Arc staining in the CA1 revealed no significant differences between groups. C) Quantification of dendritic Arc staining in the CA1 revealed group WM/RAM showed greater dendritic Arc staining compared to group WM Only. 
A

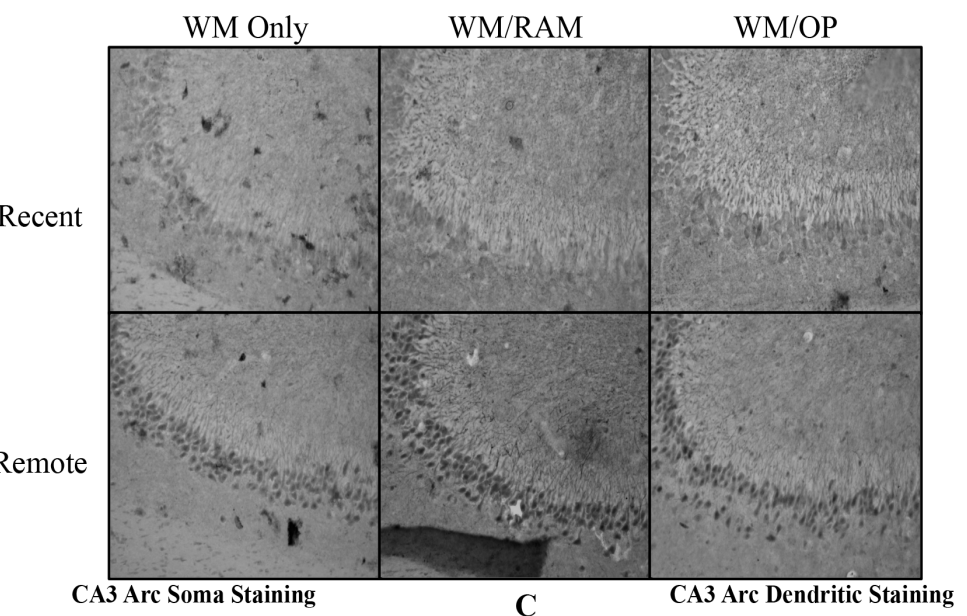

B

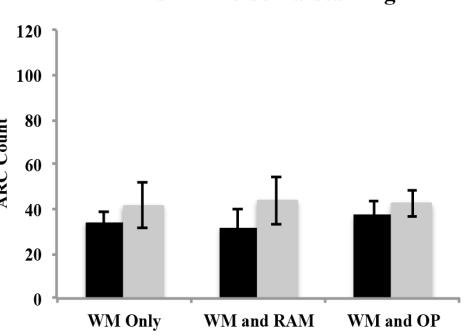

C

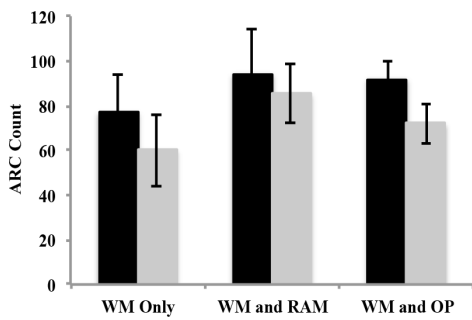

Figure 2.11: A) Representative images of Arc staining in the CA3 at 20X magnification. B) Quantification of somatic Arc staining in the CA3 revealed no significant differences between groups. C) Quantification of dendritic Arc staining in the CA3 revealed no significant differences between groups. 


\section{Manuscript 2}

The c-Fos findings from Manuscript 1 suggested that taxing the demand placed upon the hippocampus accelerated the time at which the ACC became involved in memory retrieval. Structural changes are proposed to underlie the storage and persistence of long-term memories (Bailey and Eric, 1993; Trommald et al., 1996; Yang et al., 2009). The primary purpose of this manuscript was to examine the progression of structural changes occurring in the hippocampus and ACC owing to the consolidation and storage of memories following processing, not their retrieval.

To this end, the multiple memory behavioural procedure was carried out as described in Manuscript 1, however, no probe trial followed training. We ex-

amined whether structural changes at the synaptic and systems level mimicked the activational and behavioural results found in Manuscript 1 . 


\section{Chapter 3}

\section{The impact of multiple memory formation on dendritic complexity in the hippocampus and anterior cingulate cortex assessed at recent and remote time points.}

B.C. Wartman \& M.R. Holahan

Frontiers in Behavioural Neuroscience, 2014

8:128. doi: 10.3389/fnbeh.2014.00128

Department of Neuroscience, Carleton University

Keywords: hippocampus, anterior cingulate cortex, remote memory, Golgi, memory storage 


\subsection{Abstract}

Consolidation processes, involving synaptic and systems level changes, are suggested to stabilize memories once they are formed. At the synaptic level, dendritic structural changes are associated with long-term memory storage. At the systems level, memory storage dynamics between the hippocampus and anterior cingulate cortex (ACC) may be influenced by the number of sequentially encoded memories. The present experiment utilized Golgi-Cox staining and neuron reconstruction to examine recent and remote structural changes in the hippocampus and ACC following training on three different behavioural procedures.

Rats were trained on one hippocampal-dependent task only (a water maze task), two hippocampal-dependent tasks (a water maze task followed by a radial arm maze task), or one hippocampal-dependent and one non-hippocampaldependent task (a water maze task followed by an operant conditioning task). Rats were euthanized recently or remotely. Brains underwent Golgi-Cox processing and neurons were reconstructed using Neurolucida software (MicroBrightField, Williston, VT, USA).

Rats trained on two hippocampal-dependent tasks displayed increased dendritic complexity compared to control rats, in neurons examined in both the $\mathrm{ACC}$ and hippocampus at recent and remote time points. Importantly, this behavioural group showed consistent, significant structural differences in the ACC compared to the control group at the recent time point. These findings suggest that taxing the demand placed upon the hippocampus, by training rats on two hippocampal-dependent tasks, engages synaptic and systems consolidation processes in the ACC at an accelerated rate for recent and remote storage of spatial memories. 


\subsection{Introduction}

Consolidation is distinguished into two specific components, synaptic and systems consolidation. Synaptic consolidation occurs shortly after acquisition and refers to changes at the cellular level (Alberini et al., 2006; Dudai, 2004, 2012; Frankland and Bontempi, 2005; Kandel, 2001; McGaugh et al., 2000). Systems consolidation refers to a gradual process of reorganization and rearrangement in the brain circuits which support the storage of memories, shifting the storage of memories from an initial encoding circuit to a circuit for long-term storage (Alberini et al., 2006; Dudai, 2004, 2012; Frankland and Bontempi, 2005; Kandel, 2001; McGaugh et al., 2000)

Synaptic consolidation processes involve the induction of signaling cascades and second messenger systems, protein synthesis and gene expression alterations, all of which may be important synaptic pathways in the initiation of long-term memory storage processes (Alberini et al., 2006; Dudai, 2004, 2012; Kandel, 2001). Once these pathways have been initiated, structural changes, in the form of dendritic complexity, often ensue and are proposed to provide a substrate for the storage of long-term, or remote, memories (Bailey and Eric, 1993; Trommald et al., 1996; Yang et al., 2009). Therefore, examining differences in dendritic complexity in distinct brain regions following a behavioural task may allow one to infer the involvement of that particular brain region in storing the memory representation for the task.

The hippocampus critically contributes to the initial encoding and storage of memory representations (Cohen and Eichenbaum, 1993; Scoville and Milner, 1957; Squire, 1992). It is hypothesized that as time passes, there is a shift in the balance of neural activity from the hippocampus to cortical regions in the representation of a remote memory. Evidence for the involvement of the anterior cingulate cortex (ACC) in representing remote memories is mounting. Studies have shown increased activity in the ACC on tests for remote $(\sim 30$ days after encoding) memory (Bontempi et al., 1999; Frankland et al., 2004; Lopez et al., 2012; Maviel et al., 2004; Teixeira et al., 2006; Weible et al., 2012), inactivation of the ACC has been shown to hinder performance on remote memory tests (Ding et al., 2008; Frankland et al., 2004; Holahan et al., 2007; Lopez et al., 2012; Maviel et al., 2004; Teixeira et al., 2006) and structural changes, indicative of memory storage within the ACC, have been observed at remote time points (Restivo et al., 2009; Vetere et al., 2011).

The present experiment investigated whether taxing the demand placed upon the hippocampus, by training rats on two hippocampal-dependent tasks, would alter systems consolidation process between the hippocampus and ACC. Rats were trained on one hippocampal-dependent task (the water maze; WM), two different hippocampal-dependent tasks (the WM followed by the radial arm maze; RAM) or one hippocampal-dependent and one non-hippocampaldependent task (the WM followed by operant conditioning; OP). Because impaired hippocampal function does not alter OP task performance (Carvalho et al., 2001; Corbit and Balleine, 2000; Gallagher and Hollandt, 1992; Shull and Holloway, 1985) but does impair WM (Broadbent et al., 2006; Clark et al., 
2005a,b; D'Hooge and De Deyn, 2001; Holahan and Routtenberg, 2011; Morris et al., 1986, 1982; Ramos, 2009; Remondes and Schuman, 2004; Sutherland et al., 2001, 1983; Teixeira et al., 2006; Wiltgen et al., 2010) and RAM (Bouffard and Jarrard, 1988; Jarrard, 1978; Olton and Papas, 1979; Olton et al., 1978) performance, the OP task is classified as non-hippocampal dependent and the WM and RAM are classified as hippocampal dependent. As such, sequential training on the WM and RAM tasks may result in the activation of similar neural pathways within the hippocampus and result in an accelerated systems consolidation. To examine this hypothesis, dendritic complexity was analyzed in the $\mathrm{ACC}$ and $\mathrm{CA} 1$ at recent and remote time points using the Golgi-Cox method.

\subsection{Materials \& Methods}

\subsubsection{Subjects}

Forty-eight Long Evans rats (190-250g) from Charles River, Quebec were used. Rats were housed individually in clear plastic cages (26 X 20 X 45cm) and given ad libitum water under a 12 hour light/dark cycle (lights on at 8:00 a.m.; rats tested during the light phase). Rats received no nesting material and no direct enrichment of any kind in their home cage. Food was restricted until rats reached $90 \%$ of their free-feeding baseline, which was maintained throughout the experiment. Prior to behavioural training, rats were given 5 chocolate pellets (45mg) in their home cage and handled for 5 min daily. Principles of laboratory animal care were followed and all procedures were conducted in accordance with the Canadian Council on Animal Care and protocols approved by the Carleton University Animal Care Committee.

\subsubsection{Apparatus}

\section{Water Maze (WM)}

The WM was located in a room within the animal housing area. The opaque, white, polypropylene pool measured $155 \mathrm{~cm}$ in diameter and $60 \mathrm{~cm}$ in height. The pool was filled to a depth of $37.5 \mathrm{~cm}$ with water that remained at approximately $21^{\circ} \mathrm{C}$. The escape platform was made from clear Plexiglas and submerged approximately $2 \mathrm{~cm}$ below the surface of the water. Visual cues such as posters and geometric shapes were located on the walls around the room. The experimenter remained in the same position throughout all trials.

\section{Radial Arm Maze (RAM)}

RAM testing was done in a room within the animal housing area, located across the hall from the WM testing room. The maze was positioned $98.5 \mathrm{~cm}$ off the

floor. Each arm measured $59 \mathrm{~cm}$ long and $11 \mathrm{~cm}$ wide. The distance between the ends of arms, where food (chocolate pellet, BioServe, New Jersey) reward was 
located, was $32.5 \mathrm{~cm}$. Plastic inserts were placed on the sides of the maze arms to prevent animals from jumping across arms. Visual cues such as posters and geometric shapes were located on the walls around the room. The experimenter remained in the same position throughout all trials.

\section{Operant Conditioning (OP)}

Rats were tested in groups of six using six operant chambers (Habitest Operant Cage, Coulbourn Instruments; 12 X 10 X12 inches). Each chamber was housed in an insulated box to minimize external noise. Each chamber possessed a pellet dispensing system, two levers separated by a food hopper, a houselight, a grid floor and a three light-panel. OP training took place in a room outside of the animal housing area.

\subsubsection{Behavioural Procedure}

An overview of the behavioural procedure is shown in Figure 3.1. Rats were assigned to one of three behavioural groups: (1) Training on one hippocampaldependent task, the WM; (2) Training on two different hippocampal-dependent tasks, the WM followed by the RAM; or (3) Training on one hippocampaldependent task and one non-hippocampal-dependent task, the WM followed by OP. Sequential training on two tasks was separated by a 24 hour rest period. A group of control rats received no behavioural training. Following training, rats were sacrificed recently ( 8 days after the end of WM training, $n=24$ ) or remotely (37 days after the end of WM training, $n=24$ ). Eight experimental groups resulted ( $\mathrm{n}=6$ in each group): (1) Control:Recent (2) WM:Recent (3) WM/RAM:Recent (4) WM/OP:Recent (5) Control:Remote (6) WM:Remote (7) WM/RAM:Remote (8) WM/OP:Remote.

\section{Water Maze}

Rats received 5 training trials per day for 5 days, with a different starting location for every trial within a day and randomized starting locations across days. The hidden platform was located in a fixed location within and across days. Rats were placed in the pool, facing the perimeter, and given a maximum of 60 seconds to locate the hidden platform. Rats that did not find the hidden platform within 60 seconds were guided to the platform by the experimenter. All rats remained on the platform for 15 seconds. Rats then received a 15 second rest period in a holding cage before the next trial. All movement within the pool was tracked using HVS Image 2100 Tracking System (HVS Image, Buckingham, UK). Following the final trial of each day, rats were dried with a towel and placed in a holding cage on a heating pad in the housing room for 10-15 minutes after which they were returned to the home cage. 


\section{Radial Arm Maze}

Rats received one day of pretraining and 4 days of testing on the RAM. On the first trial of pretraining, chocolate pellets were located in the starting area, at the entrance to arms, within the arms as well as in the food holes located at the end of each arm. On trial 2 of pretraining, pellets were located within the arms and in food holes only. Trials 3-5 of pretraining had pellets located only in food holes.

Days 2-5 were testing days, where pellets were located in food holes at the ends of 3 of the 5 arms. Baited arms were always the same for an individual rat but differed between rats. Each rat was given 5 trials per day. Trials were a maximum of 5 minutes each or ended when all food reward had been collected. Rats were placed in a holding cage for 30 seconds between trials while arms were re-baited. Performance on the maze was manually scored. Sessions were timed, and correct and incorrect arm entries were recorded. An arm entry was defined as all four feet inside an arm.

\section{Operant Conditioning}

Rats in the WM/OP condition were trained to lever-press for chocolate pellets over 5 days, 30 minutes per day. Upon pressing the lever to the left of the hopper two times (FR2), the house light extinguished, the panel lights above the lever changed from red to green and the pellet dispenser released one 45-mg chocolate pellet (BioServe, New Jersey) into the hopper. Presses on the right lever had no programmed consequences. Presses on the left lever were considered correct and presses on the right lever, incorrect. The number of lever presses, the number of times a rat poked its nose into the hopper and locomotor activity were recorded automatically (Graphic State Notation 2 Version 2.002-00, Coulbourn Instruments).

\subsubsection{Golgi-Cox Procedure}

The Golgi method, originally named the Black Reaction was developed by Camillo Golgi in 1873. The invention of the Golgi method marked a huge advancement in the field of neuroscience, largely thanks to Santiago Ramon y Cajals use of the method. Curiously, and fortunately, the Golgi method stains only a small percentage of cells (estimates range between 1-5\% (Milatovic et al., 2010; Spacek, 1989). This is one of the great advantages of the Golgi method: if every neuron were to become impregnated and stained, it would be impossible to trace a single neuron from its beginning to end amongst all the processes from other neurons. The seemingly random staining seen in the Golgi method provides the opportunity to examine individual neurons. Even presently, it is not well understood why some neurons become impregnated with Golgi solution and why some do not. Some factors suggested to play a role include the functional or metabolic state of a neuron at the time of Golgi fixation or mechanical injury from tissue processing rendering a neuron more susceptible 
to impregnation (Pannese, 2010; Spacek, 1989). The Golgi-Cox method used in this study is a variation on the original Golgi method. This variation uses potassium dichromate and mercuric chloride and is well-suited for analyses in rats (Gibb and Kolb, 1998; Glaser and Loos, 1981). The Golgi-Cox method is considered appropriate for the purposes of studying dendritic morphology (Buell, 1982; Ranjan and Mallick, 2010).

Rats were placed into a Decapicone (Braintree) and decapitated. Brains were rapidly removed and hemisected (hemispheres were counterbalanced). One hemisphere was placed in Golgi-Cox fixative and stored at room temperature, away from light, for 14 days. Following incubation in Golgi-Cox fixative, brains underwent 3 washes in $\mathrm{DH}_{2} \mathrm{O}$ (4 hours, 3 hours, and 8 hours; fresh $\mathrm{DH} 2 \mathrm{O}$ each time). Next, brains underwent incubation in $10 \%$ ( 8 hours), $20 \%$ ( 8 hours) and $30 \%$ sucrose (minimum four days). $200 \mu \mathrm{m}$ thick sections were sliced on a vibratome and mounted onto gelatinized slides. Slides were placed in a humidified, dark box for 24 hours before staining.

Slides were immersed in the following solutions: $\mathrm{DH}_{2} \mathrm{O}$ (1 minute), $28 \%$ Ammonium Hydroxide (40 minutes), $\mathrm{DH}_{2} \mathrm{O}$ (1 minute), Kodak film fix A (40 minutes), $\mathrm{DH}_{2} \mathrm{O}$ (2 X 1 minute), $50 \%, 70 \%$ and $95 \% \mathrm{ETOH}$ (1 min each), desiccated 100\% ETOH (3 X 5 minutes), desiccated ETOH/Clearene/Chloroform solution (10 minutes), and desiccated Clearene (2 X 15 minutes). Slides were coverslipped with generous amounts of Permount mounting medium (Sigma) and placed in a desiccated box to dry for a minimum of 4 days.

\subsubsection{Quantitative neuromorphology}

Three pyramidal neurons per rat were analyzed from each brain region. Four rats from each behavioural group were examined. All neuron reconstruction was performed by one experimenter who was unaware of the experimental condition. Brain regions were defined according to Paxinos and Watson (2006). The ACC area of interest was constricted in the anterior-posterior plan from approximately bregma +1.7 to $+0.7 \mathrm{~mm}$. The medial border was the midline of the brain and the lateral border was the corpus callosum. Neurons from layer II/III of the ACC were analyzed. The CA1 area of interest focused on the anterior aspect of the dorsal hippocampus and was constricted in the anteriorposterior plan from approximately bregma -3.1 to $-4.0 \mathrm{~mm}$.

Neurons to be traced were selected at random, but had to meet predetermined criteria for analysis. Neurons had to be entirely impregnated, staining had to be uniform and complete within processes, the neuron had to be relatively isolated from surrounding impregnated cells or obstructions, and the cell body had to be centrally located within the $200 \mu \mathrm{m}$ section depth (Anderson et al., 2009). Neurons were reconstructed at 100X magnification using Neurolucida software (MicroBrightField, Williston, VT, USA). Tracings began at the cell body. Apical and basal dendrites were traced in their entirety and all visible spines were marked. For each neuron, dendritic length, total number of branches, and total number of spines were analysed separately in apical and basal dendrites. 


\subsubsection{Statistical analyses}

Four rats from each behavioural group were examined; three neurons per rat per brain area (ACC and CA1) were analyzed. Thus resulting in 12 neurons per behavioural condition per brain region (i.e. 96 reconstructed neurons from the ACC and 96 reconstructed neurons from the CA1). For statistical analysis, the measurements of the three analyzed neurons per rat per brain region were pooled to get one value for each rat ( $\mathrm{n}=4$ per behavioural group). Separate analyses were carried out in the ACC and CA1 of the hippocampus and apical and basal dendrites were analyzed separately. Two-way ANOVAs in each region for apical and basal dendrites were run with Behavioural group (Control, WM Only, WM/RAM or WM/OP) and Time (Recent or Remote) as the fixed factors and the characteristic of interest (dendritic length, total number of branches, and total number of spines) as the dependent variables.

\subsection{Results}

\subsubsection{Behavioural training}

Figure 3.2 shows acquisition data for the WM, RAM and OP tasks. All rats received WM training. Latency to reach the hidden platform was recorded (Figure 3.2A). A repeated measures ANOVA with day (1 to 5 ) as the withinsubject factor and behaviour (WM Only, WM/RAM, or WM/OP) and time (recent or remote) as the between-subject factors revealed a main effect of day $\left(\mathrm{F}_{(4,120)}=112.88, p<0.001\right)$ but no main effect of behaviour or time.

Two groups received training on the WM followed by training on the RAM: one assigned to receive a recent WM probe test and one assigned to receive a remote WM probe (Figure 3.2B). The number of errors (unbaited arm entries and re-entries) made during training was analyzed with a repeated measures ANOVA with day (2 to 4 ) as the within-subject factor and time (recent or remote) as the between-subject factor. Analyses revealed a main effect of day $\left(\mathrm{F}_{(3,30)}=2.82, p=0.05\right)$ but no main effect of time.

Two groups received training on the WM followed by training on the operant task: one assigned to receive a recent WM probe test and one assigned to receive a remote WM probe (Figure $3.2 \mathrm{C}$ ). The number of correct lever presses made during training was analyzed with a repeated measures ANOVA with day (1 to 5) as the within-subject factor and time (recent or remote) as the betweensubject factor. Analyses revealed a main effect of day $\left(\mathrm{F}_{(4,40)}=12.73, p<0.001\right)$ but no main effect of time.

\subsubsection{Neuron morphology}

\section{Cell body size}

Cell body size in the ACC was analyzed using a two-way ANOVA with Behaviour (Control, WM Only, WM/RAM or WM/OP) and time (recent or re- 
mote) as the independent variables and cell body size as the dependent variable. No main effect of behaviour or time was found (data not shown).

Cell body size in the CA1 of the hippocampus was analyzed using a twoway ANOVA with Behaviour (Control, WM Only, WM/RAM or WM/OP) and

time (recent or remote) as the independent variables and cell body size as the dependent variable. No main effect of behaviour or time was found (data not shown).

\section{Anterior cingulate cortex}

Figure 3.3 displays images of Golgi impregnated neurons and representative graphics of Golgi impregnated neurons from each of the behavioural groups in the ACC.

\section{Number of branches}

Figure 3.4 displays total number of branches in the ACC in apical and basal dendrites.

Apical

A two-way ANOVA with Behavioural group (Control, WM Only, WM/RAM or WM/OP) and time (Recent or Remote) as the fixed factors and number of branches as the dependent variable revealed a main effect of behaviour $(\mathrm{F}(3,24)=7.46$, $p<0.001)$ with group WM/RAM displaying the greatest number of branches (16). Fishers LSD post-hoc analyses revealed group WM/RAM:Recent had significantly greater number of branches compared to groups WM/OP:Remote, Control:Recent and Control:Remote $(p<0.05)$. Group WM/RAM:Remote had significantly greater number of branches compared to groups WM Only:Recent, WM/OP:Recent, WM/OP:Remote, Control:Recent and Control:Remote $(p<0.05)$. Group WM Only:Remote had significantly greater number of branches compared to group Control:Remote $(p<0.05)$.

Basal

A two-way ANOVA with Behavioural group (Control, WM Only, WM/RAM or WM/OP) and time (Recent or Remote) as the fixed factors and number of branches as the dependent variable revealed a main effect of behaviour $\left(\mathrm{F}_{(3,24)}=5.98\right.$, $p<0.01$ ), with group WM/RAM displaying the greatest number of branches (18). Fishers LSD post-hoc analyses revealed group WM/RAM:Remote had significantly greater number of branches compared to groups from WM Only:Recent, WM Only:Remote, WM/OP:Remote, Control:Recent and Control:Remote, $(p \leq$ 0.05). Group WM/OP:Recent had significantly greater number of branches compared to group Control:Recent $p<0.05$ ).

\section{Dendritic length}

Figure 3.5 displays total dendritic length in the ACC in apical and basal dendrites. 
Apical

A two-way ANOVA with Behavioural group (Control, WM Only, WM/RAM or $\mathrm{WM} / \mathrm{OP}$ ) and time (Recent or Remote) as the fixed factors and dendritic length as the dependent variable revealed a main effect of behaviour $\left(\mathrm{F}_{(3,24)}=5.00\right.$, $p<0.01)$, with group WM/RAM displaying the greatest dendritic length $(1016 \mu \mathrm{m})$. Fishers LSD post-hoc analyses revealed group WM/RAM:Recent had significantly greater dendritic length compared to groups WM/OP:Remote, Control:Recent, and Control:Remote $(p<0.05)$. Group WM/RAM:Remote had significantly greater dendritic length compared to groups WM/OP:Remote, Control:Recent, Control:Remote $(p<0.05)$. Group WM/OP:Recent had significantly greater dendritic length compared to group Control:Remote $(p=0.05)$.

Basal

A two-way ANOVA with Behavioural group (Control, WM Only, WM/RAM or $\mathrm{WM} / \mathrm{OP}$ ) and time (Recent or Remote) as the fixed factors and dendritic length as the dependent variable revealed a main effect of behaviour $\left(\mathrm{F}_{(3,24)}=8.52\right.$, $p<0.001)$, with group WM/RAM displaying the greatest dendritic length $(839 \mu \mathrm{m})$. Fishers LSD post-hoc analyses revealed group WM/RAM:Recent had significantly greater dendritic length compared to groups WM Only:Recent, WM Only:Remote, WM/OP:Remote, Control:Recent, and Control:Remote $(p<0.05)$. Group WM/RAM:Remote had significantly greater dendritic length compared to groups WM Only:Recent, WM Only:Remote, WM/OP:Remote, Control:Recent and Control:Remote $(p<0.05)$.

\section{Number of spines}

Figure 3.6 displays total number of spines in the ACC in apical and basal dendrites.

Apical

A two-way ANOVA with Behavioural group (Control, WM Only, WM/RAM or $\mathrm{WM} / \mathrm{OP}$ ) and time (Recent or Remote) as the fixed factors and number of spines as the dependent variable revealed a main effect of behaviour $\left(\mathrm{F}_{(3,24)}=6.92\right.$, $p<0.01)$, with group WM/RAM displaying the greatest number of spines $(511)$. Fishers LSD post-hoc analyses revealed group WM/RAM:Recent had significantly greater number of spines compared to groups WM Only:Recent, WM Only:Remote, WM/OP:Recent, WM/OP:Remote, Control:Recent and Control:Remote $(p<0.05)$. Group WM/RAM:Remote had significantly greater number of spines compared to groups Control:Recent and Control:Remote $(p<0.05)$.

Basal

A two-way ANOVA with Behavioural group (Control, WM Only, WM/RAM or $\mathrm{WM} / \mathrm{OP}$ ) and time (Recent or Remote) as the fixed factors and number of spines as the dependent variable revealed a main effect of behaviour $\left(\mathrm{F}_{(3,24)}=10.23\right.$, $p<0.001$ ), with group WM/RAM displaying the greatest number of spines (385). Fishers LSD post-hoc analyses revealed group WM/RAM:Recent had significantly greater number of spines compared to groups WM Only:Recent, 
WM Only:Remote, WM/OP:Recent, WM/OP:Remote, Control:Recent, Control:Remote $(p<0.05)$. Group WM/RAM:Remote had significantly greater number of spines compared to groups WM Only:Recent, WM Only:Remote, Control:Recent and Control:Remote $(p<0.05)$. Group WM/OP:Recent had significantly greater number of spines compared to groups WM Only:Recent and Control:Recent $(p<0.05)$.

\section{Spine density}

Figure 3.7 displays spine density in the ACC in apical and basal dendrites.

Apical

A two-way ANOVA with Behavioural group (Control, WM Only, WM/RAM or $\mathrm{WM} / \mathrm{OP}$ ) and time (Recent or Remote) as the fixed factors and spine density as the dependent variable revealed no main effects. Planned Fishers LSD post-hoc comparisons revealed group WM/RAM:Recent had significantly greater spine density compared to group Control:Recent $(p<0.05)$.

Basal

A two-way ANOVA with Behavioural group (Control, WM Only, WM/RAM or WM/OP) and time (Recent or Remote) as the fixed factors and spine density as the dependent variable revealed no main effects. A significant interaction between behaviour $\mathrm{X}$ time $\left(\mathrm{F}_{(3,24)}=3.39, p<0.05\right)$ was found. Fishers LSD post-hoc comparisons revealed group WM/RAM:Recent had significantly greater spine density compared to groups WM Only:Recent, WM Only:Remote, WM/RAM:Remote and Control:Recent $(p<0.05)$. Group WM/OP:Recent had significantly greater spine density compared to group Control:Recent $(p<0.05)$. Group Control:Remote had significantly greater spine density compared to group Control:Recent $(p<0.05)$.

\section{Summary of ACC findings}

Group WM/RAM displayed the greatest and most consistent dendritic complexity in the ACC. At the recent time point, group WM/RAM was the sole group to show significantly increased dendritic complexity in apical dendrites compared to the control group.

\section{CA1 of the hippocampus}

Figure 3.8 displays images of Golgi impregnated neurons and representative graphics of Golgi impregnated neurons from each of the behavioural groups in the CA1.

\section{Number of branches}

Figure 3.9 displays total number of branches in the CA1 in apical and basal dendrites.

Apical 
A two-way ANOVA with Behavioural group (Control, WM Only, WM/RAM or WM/OP) and time (Recent or Remote) as the fixed factors and number of branches as the dependent variable revealed a main effect of behaviour $\left(\mathrm{F}_{(3,24)}=12.63\right.$, $p<0.001)$ with group WM/RAM displaying the greatest number of branches (38). Fishers LSD post-hoc analyses revealed group WM/RAM:Recent had significantly greater number of branches compared to every other behavioural group $(p<0.05)$. Group WM/RAM:Remote had significantly greater number of branches compared to groups WM Only:Recent, WM Only:Remote, WM/RAM:Recent, Control:Recent and Control:Remote $(p<0.05)$. Group WM/OP:Recent had significantly greater number of branches compared to group WM/OP:Remote $(p<0.05)$. Group WM/OP:Remote had significantly greater number of branches compared to groups WM/RAM:Recent, Control:Recent and Control:Remote $(p<0.05)$.

\section{Basal}

A two-way ANOVA with Behavioural group (Control, WM Only, WM/RAM or WM/OP) and time (Recent or Remote) as the fixed factors and number of branches as the dependent variable revealed a main effect of behaviour $\left(\mathrm{F}_{(3,24)}=2.98\right.$, $p=0.05)$ with group WM/RAM displaying the greatest number of branches (16). A significant interaction between behaviour $\mathrm{X}$ time was found $\left(\mathrm{F}_{(3,24)}=3.42\right.$, $p<0.05)$. Fishers LSD post-hoc analyses revealed group WM/RAM:Remote had significantly greater number of branches compared to groups WM Only:Remote, WM/OP:Recent, Control:Recent and Control:Remote $(p<0.05)$. Group WM/OP:Recent had significantly fewer number of branches compared to groups WM/RAM:Remote, WM/OP:Remote $(p<0.05)$. Group WM/OP:Remote had significantly greater number of branches compared to group WM Only:Remote, WM/OP:Recent and Control:Recent $(p<0.05)$.

\section{Dendritic length}

Figure 3.10 displays total dendritic length in the CA1 in apical and basal dendrites.

\section{Apical}

A two-way ANOVA with Behavioural group (Control, WM Only, WM/RAM or $\mathrm{WM} / \mathrm{OP}$ ) and time (Recent or Remote) as the fixed factors and dendritic length as the dependent variable revealed a main effect of behaviour $\left(\mathrm{F}_{(3,24)}=6.24\right.$, $p<0.01)$, with group WM/RAM displaying the greatest dendritic length $(2259 \mu \mathrm{m})$. A significant interaction between behaviour $\mathrm{X}$ time was found $\left(\mathrm{F}_{(3,24)}=4.04\right.$, $p<0.05)$. Fishers LSD post-hoc analyses revealed group WM/RAM:Recent had significantly greater dendritic length compared to groups WM Only:Recent, WM Only:Remote, WM/OP:Recent, Control:Recent and Control:Remote $(p<0.05)$. Group WM/RAM:Remote had significantly greater dendritic length compared to group Contol:Remote $(p<0.05)$. Group WM/OP:Remote had significantly greater dendritic length compared to groups WM Only:Recent, WM Only:Remote, WM/OP:Recent, Control:Recent and Control:Remote $(p<0.05)$. 
Basal

A two-way ANOVA with Behavioural group (Control, WM Only, WM/RAM or $\mathrm{WM} / \mathrm{OP}$ ) and time (Recent or Remote) as the fixed factors and dendritic length as the dependent variable revealed no main effects. Planned Fishers LSD posthoc comparisons revealed group WM/OP:Remote had significantly greater dendritic length compared to groups WM Only:Remote and Control:Recent. Group Control:Recent had significantly less dendritic length compared to groups WM Only:Recent and WM/OP:Remote $(p<0.05)$.

\section{Number of spines}

Figure 3.11 displays total number of spines in the CA1 in apical and basal dendrites.

\section{Apical}

A two-way ANOVA with Behavioural group (Control, WM Only, WM/RAM or $\mathrm{WM} / \mathrm{OP}$ ) and time (Recent or Remote) as the fixed factors and number of spines as the dependent variable revealed a main effect of behaviour $\left(\mathrm{F}_{(3,24)}=10.81\right.$, $p<0.001$ ), with group WM/RAM displaying the greatest number of spines (1793). Fishers LSD post-hoc analyses revealed group WM/RAM:Recent had significantly greater number of spines compared to groups WM Only:Recent, WM Only:Remote, WM/OP:Recent, Control:Recent and Control:Remote $(p<0.05)$. Group WM/RAM:Remote had significantly greater number of spines compared to group WM Only:Recent, WM Only:Remote, WM/OP:Reent, Control:Recent and Control:Remote $(p<0.05)$. Group WM/OP:Recent had significantly fewer number of spines compared to groups WM/RAM:Recent, WM/RAM:Remote and WM/OP:Remote (p;0.05). Group WM/OP:Remote had significantly greater number of spines compared to groups WM Only:Recent, WM Only:Remote, WM/OP:Recent, Control:Recent and Control:Remote $(p<0.05)$.

\section{Basal}

A two-way ANOVA with Behavioural group (Control, WM Only, WM/RAM or $\mathrm{WM} / \mathrm{OP}$ ) and time (Recent or Remote) as the fixed factors and number of spines as the dependent variable revealed a main effect of behaviour $\left(\mathrm{F}_{(3,24)}=5.07\right.$, $p<0.01$ ), with group WM/RAM displaying the greatest number of spines (647). Fishers LSD post-hoc analyses revealed group WM/RAM:Recent had significantly greater number of spines compared to group Control:Recent $(p<0.05)$. Group WM/RAM:Remote had significantly greater number of spines compared to groups WM Only:Remote, WM/OP:Recent, Control:Recent and Control:Remote $(p<0.05)$. Group WM/OP:Recent had significantly fewer number of spines compared to groups WM/RAM:Remote and WM/OP:Remote $(p<0.05)$. Group WM/OP:Remote had significantly greater number of spines compared to groups WM Only:Remote, WM/OP:Recent, Control:Recent and Control:Remote $(p<0.05)$.

\section{Spine density}

Figure 3.12 displays spine density in the CA1 in apical and basal dendrites. 
Apical

A two-way ANOVA with Behavioural group (Control, WM Only, WM/RAM or WM/OP) and time (Recent or Remote) as the fixed factors and spine density as the dependent variable revealed a main effect of behaviour $\left(\mathrm{F}_{(3,24)}=3.42\right.$, $p<0.05)$, with group WM/RAM displaying the greatest spine density (0.812). Fishers LSD post-hoc analyses revealed group WM/RAM:Remote had significantly greater spine density compared to groups WM Only:Recent, WM Only:Remote, WM/OP:Recent, Control:Recent and Control:Remote $(p<0.05)$.

Basal

A two-way ANOVA with Behavioural group (Control, WM Only, WM/RAM or WM/OP) and time (Recent or Remote) as the fixed factors and spine density as the dependent variable revealed a main effect of behaviour $\left(\mathrm{F}_{(3,24)}=3.63\right.$, $p<0.05)$, with group WM/RAM displaying the greatest spine density (0.812). Fishers LSD post-hoc analyses revealed group WM/RAM:Recent had significantly greater spine density compared to group Control:Recent $(p<0.05)$. Group WM/RAM:Remote had significantly greater spine density compared to groups WM/OP:Recent and Control:Recent $(p<0.05)$.

\section{Summary of CA1 findings}

Group WM/RAM displayed the greatest dendritic complexity in the CA1. Group WM/RAM was the only group to show increased dendritic complexity at the recent time point. Group WM/RAM also showed persistent increases in the dendritic complexity characteristics analysed as this group was found to have these characteristics significantly increased from the Control group at the remote time point as well. Group WM/OP:Remote consistently showed increases in dendritic complexity at the remote time point.

\subsection{Discussion}

A great deal of human research supports the idea of a time-limited role for the hippocampus in memory storage (Bayley et al., 2006; Kapur and Brooks, 1999; Manns et al., 2003; Rosenbaum et al., 2000; Scoville and Milner, 1957; Teng and Squire, 1999). Within animal research, some evidence suggests hippocampal damage results in temporally graded retrograde amnesia (Ding et al., 2008; Frankland et al., 2004; Kim and Fanselow, 1992; Maviel et al., 2004; Teixeira et al., 2006) whereas other evidence suggests hippocampal damage results in a flat, or ungraded, retrograde amnesia (Broadbent et al., 2006; Clark et al., 2005a,b, 2007; Epp et al., 2008; Lehmann et al., 2007, 2013; Mumby et al., 1999; Sparks et al., 2011; Sutherland et al., 2008, 2001). We propose that the number of memories processed over the lifetime underlies this discrepancy. In humans, there is a seemingly endless sequence of memory storage and retrieval processes, while laboratory rats and mice are typically required to retrieve and store only one memory for one behavioural task. In the absence of multiple memories competing for processing space in the hippocampus, a hippocampal-dependent 
memory may not be as likely to require, and thus form, extra-hippocampal (cortical) connections, thereby relying heavily on hippocampal involvement for an extended period of time. Processing of several hippocampal-dependent memories (resulting from multiple hippocampal-dependent tasks) may compete for hippocampal processing space, resulting in an increased reliance on cortical structures, such as the ACC.

Dendrites represent structures that serve to receive and integrate information (Jan and Jan, 2001). As such, structural changes in dendrites may partially fulfill the neural requirements necessary to maintain memory representations for the long term (Bailey and Eric, 1993; Trommald et al., 1996). Changes in the dendritic characteristics examined (dendritic length, branching, number of spines and spine density) collectively grouped as dendritic complexity, serve to increase the available surface area for synaptic contact, increasing the capacity for neuronal connectivity (Cajal, 1909). Greater dendritic length and greater dendritic branching increase the number of possible synaptic contacts and information that may be received by a dendrite (Jan and Jan, 2001). Alterations in dendritic characteristics may reflect increased or decreased responses to incoming synaptic information.

Spine remodelling, which includes both spine formation and elimination, has been suggested to play an important role in structural plasticity (Yang et al., 2009). Yang et al. (2009) demonstrated that only a small proportion of new spines that result from novel experiences persist and survive. Thus, number of spines or spine density may not always be an effective indicator of memory storage or retention on its own; spine formation may represent a transient event following experience. Likely, the type of spine (thin, mushroom, etc) is of greater importance for memory persistence. Unfortunately, spine type was not included in the analysis in the present study as technical limitations prohibited this type of examination.

\subsubsection{Results}

Examination of neuron morphology included total dendritic length, number of branches, number of spines and spine density (Hamilton et al., 2010) in the ACC and CA1. Overall, group WM/RAM displayed the most robust increases on all indices evaluated compared to the control groups in both the ACC and CA1. This suggests a greater degree of dendritic complexity in this group. Importantly, group WM/RAM consistently showed increased dendritic complexity at the recent time point in the ACC.

In the ACC, group WM/RAM displayed longer apical and basal dendritic length and more spines, both recently and remotely, compared to the control groups. This group also displayed more branches and increased spine density compared to the control groups. Group WM/OP showed increases across most of the characteristics examined at the recent time point in the ACC, but only in basal dendrites. Group WM Only did not show increased dendritic complexity at the recent time point in the ACC. However, this group did show an increase in the number of apical branches at the remote time point. 
In the CA1, group WM/RAM displayed more apical and basal spines, both recently and remotely, compared to the control groups. This group displayed longer dendritic length and more branches and spine density compared to control groups. Group WM/OP showed increases across most of the characteristics analyzed, but only at the remote time point. Group WM Only did not show increased dendritic complexity compared to the control group.

In examining published findings on dendritic complexity, it is important to note that there is a great deal of variability between laboratories (Scorcioni et al., 2004). For example, average apical dendritic lengths in the ACC in other published reports range from $8001500 \mu \mathrm{m}$ (Bock et al., 2005; Murmu et al., 2006; Radley et al., 2004; Zehle et al., 2007). The apical dendritic length in the present report ranged from $230-626 \mu \mathrm{m}$. This variance may result from age or strain differences, tissue processing protocols or neuron reconstruction software and techniques.

These results suggest competing hippocampal-dependent memories may result in more pronounced, or accelerated, increases in dendritic complexity in the ACC. Without hippocampal-dependent memories competing for hippocampal processing space, structural changes may be less distinct, or take a greater amount of time to come about. Interestingly, group WM/OP showed increases in dendritic complexity in both the ACC and CA1. As the type of OP task used in this study is not typically dependent upon the hippocampus (Carvalho et al., 2001; Corbit and Balleine, 2000; Gallagher and Hollandt, 1992; Shull and Holloway, 1985) we presume that there is no immediate competition between memory traces for the WM and OP tasks. However, structural changes indicative of memory storage did present themselves, suggesting that multiple memory processing may result in accelerated and/or more distinct structural changes compared to the processing of just a single memory.

The results presented in this study provide new information regarding structural changes associated with memory storage. Past studies have focused on spine density as the primary measure of structural changes (Restivo et al., 2009; Vetere et al., 2011). Specifically, Restivo et al. (2009) observed an increase in spine density in the CA1 recently, but not remotely, while an increase in spine density was observed remotely, but not recently, in the ACC. Spine density was also examined in the present experiment. Significantly increased apical spine density was noted in group WM/RAM:Recent compared to Control:Recent. In basal dendrites, group WM/RAM:Recent had a significantly higher spine density compared to Control:Recent, the WM Only groups and group WM/RAM:Remote. In the CA1, apical spine density was higher in group WM/RAM:Remote compared to Control:Remote, while basal spine density was higher in group WM/RAM:Recent compared to Control:Recent.

Our results are not consistent with the pattern of spine density found by others. A few important differences between Restivo et al. (2009), Vetere et al. (2011) and the present study should be pointed out. Firstly, the time point described as recent differed substantially between the current experiment and those of Restivo et al. (2009) and Vetere et al. (2011). In the present experiment, we defined recent as 8 days following the end of WM training. Restivo 
and Vetere defined recent as 24 hours following conditioning. These represent two very different time points. It may be the case that our recent time point comes too late to see an increase in CA1 spine density recently, and a decrease remotely as has been noted before (Restivo et al., 2009; Vetere et al., 2011). Different behavioural training procedures were also used. Restivo et al. (2009) and Vetere et al. (2011) trained mice using a contextual fear conditioning procedure, while the present study trained rats using a water maze procedure (along with the RAM and OP in some groups). Differences in the type and duration of behavioural procedures may play a role as well. Our behavioural training procedure took 5 days (for each procedure) to train, whereas training on contextual fear conditioning can be done in a single day. This may result in differences in the timing of structural changes.

In the present study, structural changes were found in both the ACC and CA1 of the hippocampus. The only experimental difference between the different groups of rats was the behavioural training procedures they received. No probe trial was given prior to sacrifice. Thus the morphological changes observed were not due to retrieval processes but likely associated with a memory storage process. Group WM/RAM was the only group to show consistent, significant differences in dendritic complexity at the recent time point compared to the control group in the ACC. This finding supports the hypothesis that taxing the demand placed upon the hippocampus, by training rats on two hippocampaldependent tasks, results in structural changes indicative of memory storage, in the ACC. This is consistent with results from Wartman and Holahan (2013) that showed increased number of c-Fos positive cells in remotely probed groups compared to recently probed groups. In that report, the group trained on two hippocampal-dependent tasks (WM/RAM) and probed recently demonstrated increased c-Fos labeling in the ACC compared to the other recently probed groups, and similar to the level of c-Fos labeling noted in remotely probed groups, suggesting an accelerated reliance on the ACC.

The current study did not examine structural changes associated with training on the RAM or OP tasks alone. In future studies it would be of interest to examine the structural changes resulting from these tasks alone or reversed training on these tasks (i.e. RAM or OP training followed by WM). Future behavioural studies utilizing one-day training procedures are underway so that early structural changes in the ACC and CA1 can be examined. A one-day training procedure would also help to diminish delay differences between the end of training on a behavioural task and neuron analysis. In the present study, there was a difference in the delay between the end of behavioural training and neuron analysis in rats trained on one or two tasks. Training on the RAM or OP tasks entailed five days of training followed by a day of rest then sacrifice. Rats trained on only the WM task had six days of rest prior to sacrifice. This difference could influence dynamic dendritic characteristics such as spine density. This difference may become more substantial when examining recent versus remote time points. 


\subsubsection{Conclusion}

Neuron reconstruction data showed robust increases in dendritic complexity in the ACC and CA1 at recent and remote time points in groups trained on two hippocampal-dependent tasks. These results suggest competing hippocampaldependent memories may result in more pronounced, or accelerated, increases in dendritic complexity in the ACC. Without hippocampal-dependent memories competing for hippocampal processing space, structural changes may be less distinct, or take a longer amount of time to come about. The introduction of a multiple memory behavioural procedure presents an innovative method through which structural changes associated with memory storage can be ex-

amined. Introducing variations in the type and number of tasks used during training provides an opportunity to examine and contrast morphological differences throughout the consolidation process, shedding light on the processes underlying remote memory storage. 


\section{Supplemental material not included in Manuscript 2}

\subsection{Supplemental Introduction}

The growth-and plasticity-associated protein (GAP-43) is a presynaptic protein involved in axon growth, and is used as a marker of newly formed synapses (Benowitz, Larry and Routtenberg, 1997). GAP-43 is characterized as: a) neuron-specific (Alexander et al., 1988; Basi et al., 1987; Chan et al., 1986; Liu and Storm, 1989; Nielander et al., 1990; Skene, 1989; Zuber et al., 1989) b) in high concentrations in growth cones (Nelson et al., 1989), c) closely associated with regenerative and developmental growth (Skene, 1989; Strittmatter et al., 1995), d) having both a calmodulin-binding domain and PKC phosphorylation site (Alexander et al., 1988; De Graan et al., 1990), e) having a direct relation between its phosphorylation state and enhancement of LTP (Gianotti et al., 1992; Lovinger et al., 1985; Lovinger and Routtenberg, 1988) and f) linked to behavioural learning (Ehrlich et al., 1977; Ehrlich and Routtenberg, 1974; Pascale et al., 2004; Routtenberg et al., 2000; Routtenberg and Ehrlich, 1975; Young et al., 2002, 2000). These characteristics make GAP-43 an attractive substrate for mediating the altered state of a synapse following memory formation. More relevant to the current work, GAP-43 levels have been found to exhibit region specific increases in the ACC at remote time points following behavioural training (Maviel et al., 2004; Vetere et al., 2012). It has been suggested that high levels of GAP-43 may be associated with a neurons ability to undergo activity-dependent structural changes. The present study examined GAP-43 expression in the hippocampus and ACC at recent and remote time points following behavioural training.

\subsection{Supplemental Materials \& Methods}

Subjects and behavioural procedures were as those outlined in Manuscript 2. ACC GAP-43 concentration was measured using an ELISA kit (Biomatik).

\subsubsection{Enzyme-linked immunosorbent assay (ELISA) Sample preparation}

The ACC was dissected out and rinsed in ice-cold 0.01M phosphate buffered saline (PBS). The tissue was homogenized in $300 \mu \mathrm{L}$ PBS using a mechanical pestle. The suspension was frozen and thawed twice to aid in cell membrane breakdown. Homogenates were centrifuged for 5 minutes at $5000 \mathrm{Xg}$. The supernatant was removed and kept for use and the pellet was discarded.

\section{Reagent preparation}

All kit components were brought to room temperature before use. The standard was reconstituted with $1.0 \mathrm{~mL}$ of standard diluent to a concentration of 20 
$\mathrm{ng} / \mathrm{mL}$. Serial dilutions were made from this stock solution at concentrations of $10 \mathrm{ng} / \mathrm{mL}, 5 \mathrm{ng} / \mathrm{mL}, 2.5 \mathrm{ng} / \mathrm{mL}, 1.25 \mathrm{ng} / \mathrm{mL}, 0.625 \mathrm{ng} / \mathrm{mL}, 0.312 \mathrm{ng} / \mathrm{mL}, 0.156$ $\mathrm{ng} / \mathrm{mL}$. A blank was also made $(0 \mathrm{ng} / \mathrm{mL})$ containing only standard diluent. Detection Reagent A and B were diluted to a working concentration of 1:100 with Assay Diluent A and B respectively. The Wash Solution concentrate (30X) was diluted to a working concentration of $1 \mathrm{X}$ with distilled water.

\section{Assay procedure}

$100 \mu \mathrm{L}$ of the standard, blank and samples were loaded into the wells in duplicate. The plate was covered with a plate sealer and incubated for two hours at $37^{\circ} \mathrm{C}$ on a Jitterbug shaker. Following incubation, the plate was removed, turned upside down and tapped so the liquid from the wells was removed. $100 \mu \mathrm{L}$ of Detection Reagent A solution was added to each well. The plate was covered with a plate sealer and incubated for one hour at $37^{\circ} \mathrm{C}$ on a Jitterbug. Following incubation, the plate was removed, turned upside down and tapped so the liquid from the wells was removed. The wells were washed three times with $300 \mu \mathrm{L}$ of wash buffer. $100 \mu \mathrm{L}$ of Detection Reagent B solution was added to each well. The plate was covered with a plate sealer and incubated for thirty minutes at $37^{\circ} \mathrm{C}$ on a Jitterbug. Following incubation, the plate was removed, turned upside down and tapped so the liquid from the wells was removed. The wells were washed five times with $300 \mu \mathrm{L}$ of wash buffer. $90 \mu \mathrm{L}$ of TMB substrate solution was added to each well. The plate was covered with a plate sealer and incubated for 20 minutes at $37^{\circ} \mathrm{C}$ on a Jitterbug shaker. Following the addition of substrate solution the plate was protected from light. The liquid turned blue. $50 \mu \mathrm{L}$ of stop solution was added to the wells prior to the liquid turning a deep blue color. The addition of the stop solution caused the liquid to turn yellow in color. The stop solution was mixed in the wells by tapping the plate lightly. Following the addition of the stop solution, the plate was immediately run on a plate reader at $450 \mathrm{~nm}$.

\subsection{Supplemental Results}

Figure 3.13 displays GAP-43 concentration levels. A 4X2 (Behaviour X Time) ANOVA was run on GAP-43 concentration findings from pooled ACC tissue. No main effect of behaviour $\left(\mathrm{F}_{(3,16)}=1.52, p<0.05\right)$ time $\left(\mathrm{F}_{(1,16)}=1.95\right.$, textitp $<0.05)$, and no significant interaction between the two were found $\left(\mathrm{F}_{(3,16)}=1.31\right.$, $p<0.05)$. Planned post-hoc comparisons revealed group WM Only as the sole group that showed a difference in GAP-43 levels between recent and remote time points (Fishers post-hoc $p<0.05)$.

\subsection{Supplemental Discussion}

GAP-43 concentration was examined in the brain as it has been suggested that GAP-43 levels are related to the ability of neurons to undergo synaptic re- 
organization and structural changes (Benowitz, Larry and Routtenberg, 1997; Vetere et al., 2012). Increased GAP-43 expression in the ACC has been noted in animals tested remotely on memory tasks (Maviel et al., 2004; Vetere et al., 2012). In the present experiment, rats received training on one hippocampaldependent, two hippocampal-dependent, or one hippocampal-dependent and one non-hippocampal-dependent task and were sacrificed recently or remotely. Importantly, rats were not subjected to a memory test prior to sacrifice. Presumably, this allowed us to examine GAP-43 levels in the ACC resulting from consolidation.

The analysis of GAP-43 levels in the ACC revealed similar levels in control rats (naïve) and trained rats. No main effect of time was found. Interestingly, planned Fishers LSD post-hoc analyses revealed group WM Only:Remote had significantly higher levels of GAP-43 compared to group WM Only:Recent. Elevated GAP-43 levels have been reported in the ACC at remote compared to recent time points following a memory test (Maviel et al., 2004; Vetere et al., 2012). Our findings regarding the WM Only recent and remote groups are in line with this. The other behavioural groups tested did not follow this pattern. This may be indicative of an accelerated shift in reliance upon the ACC for memory storage when multiple memories are competing for hippocampal processing space (Wartman and Holahan, 2013), such that at the recent time point, elevated levels of GAP-43 are already present in the ACC. However, in the current study, GAP-43 levels in groups WM/RAM:Recent and WM/OP:Recent were not significantly higher than GAP-43 levels in WM Only:Recent.

ACC samples were pooled together to ensure that the ELISA kit could properly detect GAP- 43 concentrations. Thus, only three samples were run for each group. As a result of the low number of samples run, the significant increase found in the WM Only recent group compared to the WM Only remote group should be analyzed cautiously, as it could represent a spurious finding. The robustness of this result should be determined by replicating the experiment presented here with a larger number of rats per group. Based on the expression levels of GAP-43 from this experiment, pooling of samples need not be necessary in the future.

It has been suggested that phosphorylated GAP-43 is associated with the induction of LTP, but not its maintenance (Son et al., 1997), and thus was not of interest to us in this behavioural design. Vetere et al. (2012) suggested increased GAP-43 levels may represent the capacity of a neuron to participate in activity-dependent structural changes, while the state of GAP-43 phosphorylation may stipulate the direction of the structural change. Son et al. (1997) found phosphorylation of GAP-43 was increased one and five minutes following LTP induction in the CA3 of the hippocampus, but was not increased at 60 minutes. As such, we did not expect to find differences from basal levels of GAP-43 phosphorylation given that no probe test was administered prior to sacrifice. It would be interesting to examine levels of phosphorylated GAP-43 between the different behavioural groups when a probe test is delivered prior to sacrifice at recent and remote time points. Comparison of the regional expression of phosphorylated GAP-43 in the hippocampus and ACC would be interesting to 
examine as well. 


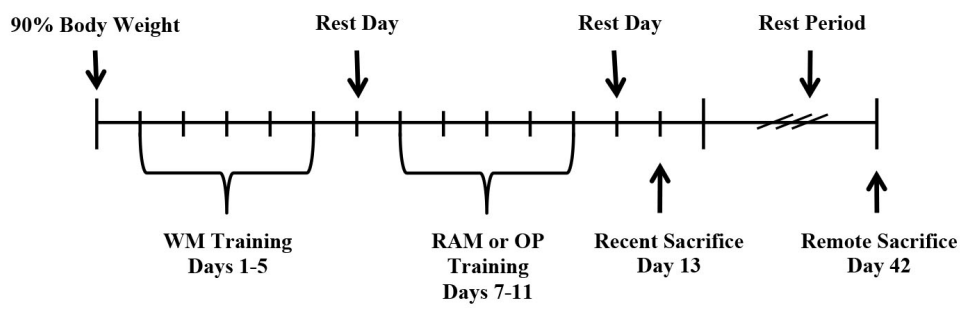

Figure 3.1: Timeline of experimental procedures 
A

Water Maze Training Latency

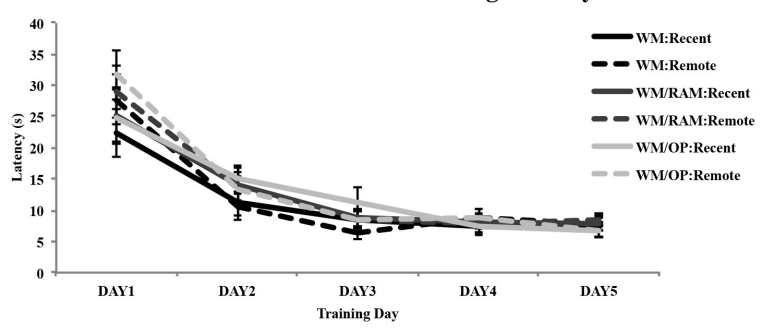

B

C
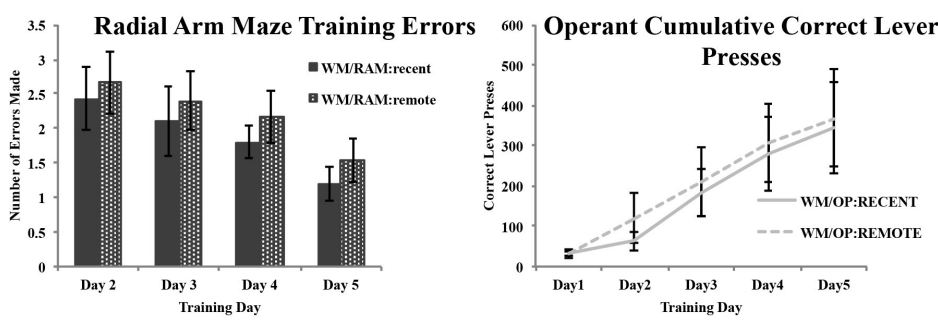

Figure 3.2: Behavioural training data. A) All rats received water maze training. Average latencies to reach the hidden platform during the five days of water maze training are displayed. A main effect of day was found. Latency to reach the hidden platform decreased across training day indicating an improvement in performance. No main effect of behaviour or time was found. B) Average number of errors (unbaited arm entries and re-entries) made in the radial arm maze task during the four days of training. A main effect of day was found. Errors made decreased across training day indicating an improvement in performance. No main effect of time was found. C) Average correct lever presses on the operant conditioning task during the five days of training. A main effect of day was found. Correct lever presses increased across training day indicating an improvement in performance. No main effect of time was found. 


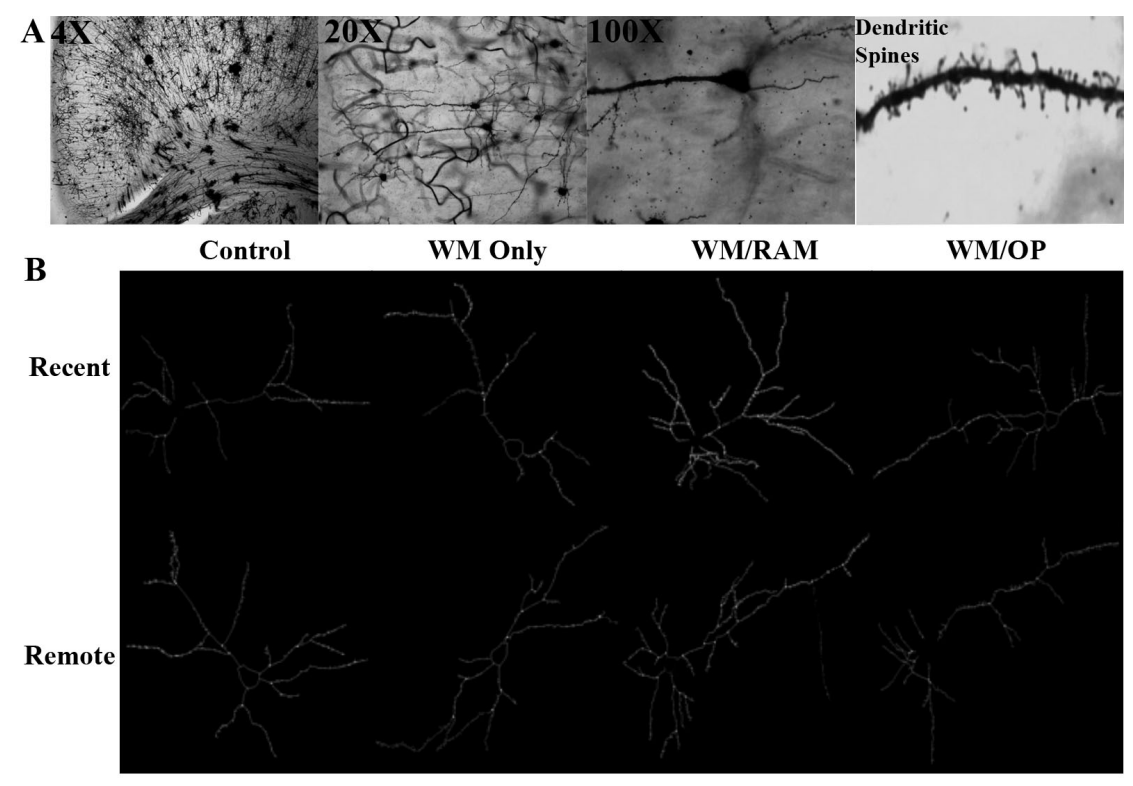

Figure 3.3: A) Images of 4X, 20X and 100X magnified Golgi impregnated neurons in the ACC. A zoomed-in image of an apical dendrite with dendritic spines is shown in the last panel. B) Representative graphics of Golgi impregnated neurons in the ACC, traced using Neurolucida software. 

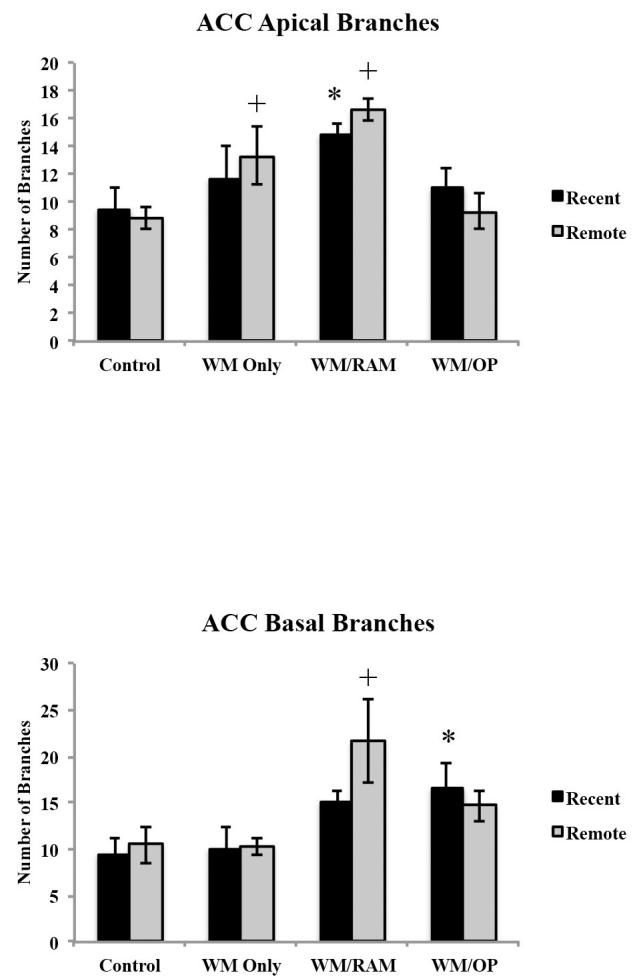

Figure 3.4: Total number of branches in the ACC in apical and basal dendrites. Only significant differences between behavioural groups and their respective control group are displayed. Refer to Results section for all comparison results. * Significantly different from Control:Recent group $(p<0.05) ;+$ Significantly different from Control:Remote $(p<0.05)$. 

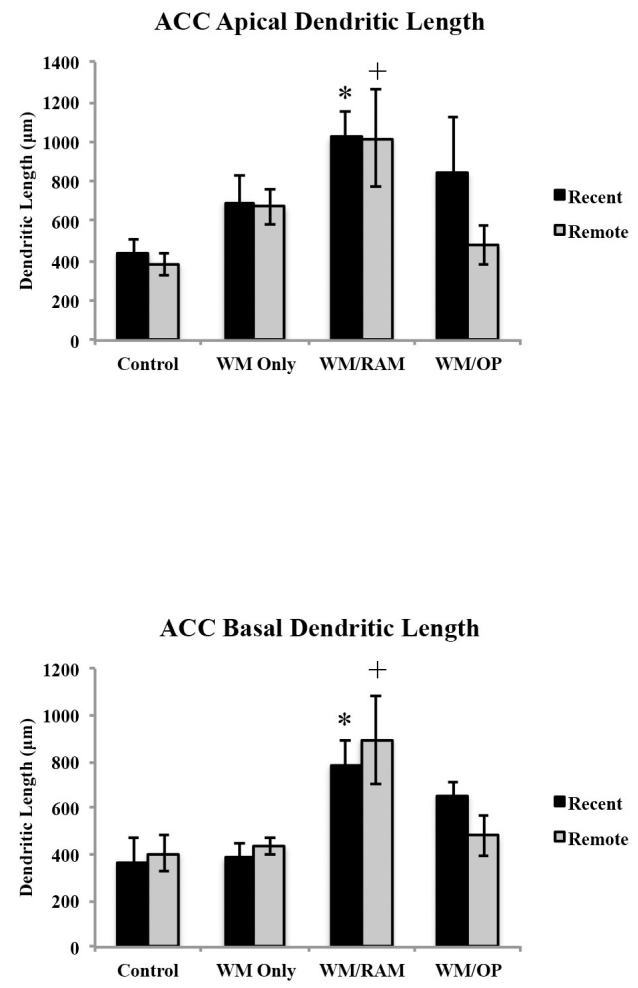

Figure 3.5: Total dendritic length in the ACC in apical and basal dendrites. Only significant differences between behavioural groups and their respective control group are displayed. Refer to Results section for all comparison results. * Significantly different from Control:recent group $(p<0.05) ;+$ Significantly different from Control:Remote $(p<0.05)$. 

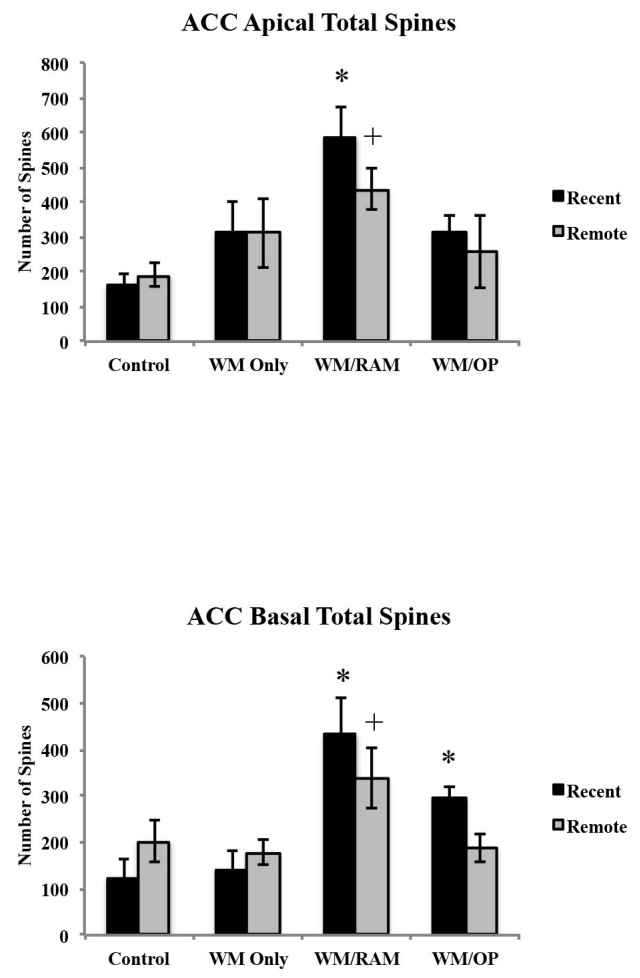

Figure 3.6: Total number of spines in the ACC in apical and basal dendrites. Only significant differences between behavioural groups and their respective control group are displayed. Refer to Results section for all comparison results. * Significantly different from Control:recent group $(p<0.05) ;+$ Significantly different from Control:Remote $(p<0.05)$. 

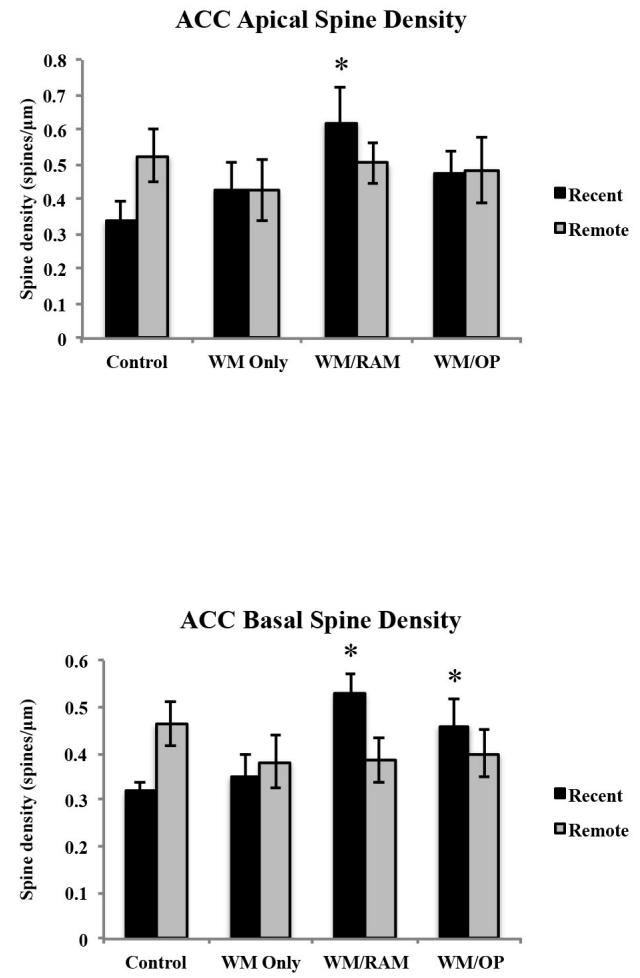

Figure 3.7: Spine density in the ACC in apical and basal dendrites. Only significant differences between behavioural groups and their respective control group are displayed. Refer to Results section for all comparison results. * Significantly different from Control:recent group $(p<0.05)$; + Significantly different from Control:Remote $(p<0.05)$. 


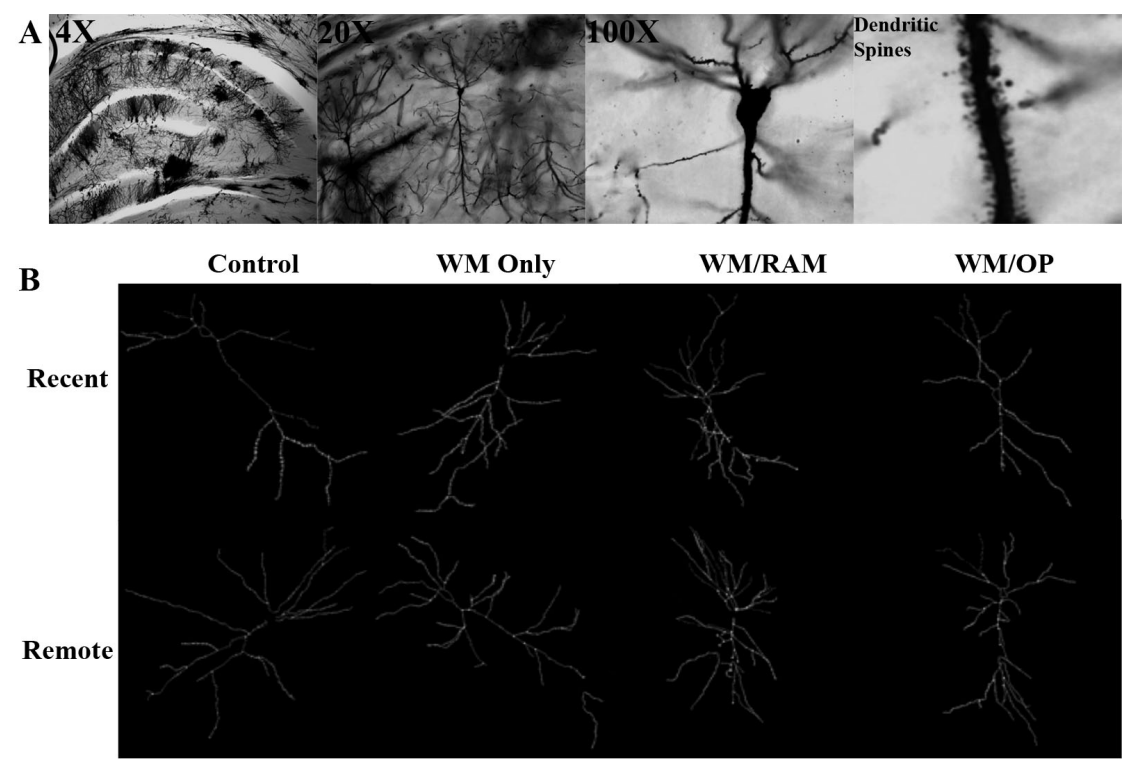

Figure 3.8: A) Images of 4X, 20X and 100X magnified Golgi impregnated neurons in the CA1. A zoomed-in image of an apical dendrite with dendritic spines is shown in the last panel. B) Representative graphics of Golgi impregnated neurons in the CA1, traced using Neurolucida software. 

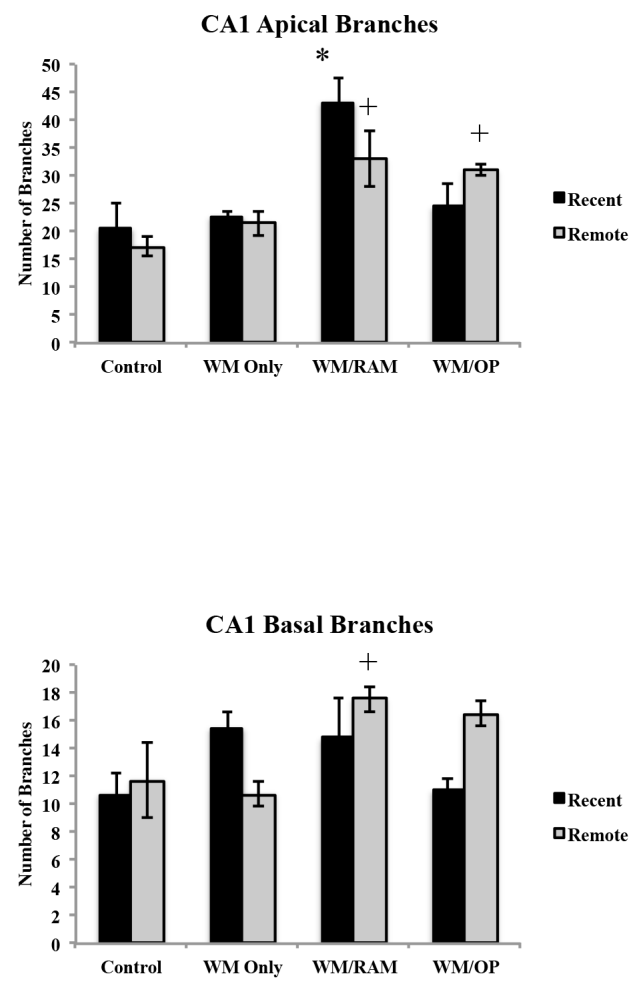

Figure 3.9: Total number of branches in the CA1 in apical and basal dendrites. Only significant differences between behavioural groups and their respective control group are displayed. Refer to Results section for all comparison results. * Significantly different from Control:recent group $(p<0.05) ;+$ Significantly different from Control:Remote $(p<0.05)$. 

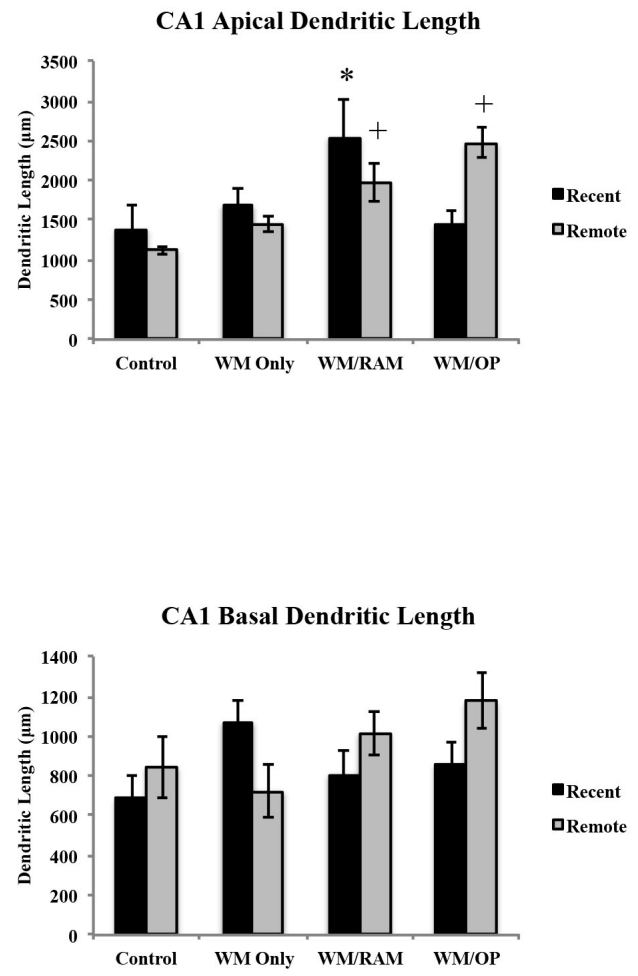

Figure 3.10: Total dendritic length in the CA1 in apical and basal dendrites. Only significant differences between behavioural groups and their respective control group are displayed. Refer to Results section for all comparison results. * Significantly different from Control:recent group $(p<0.05) ;+$ Significantly different from Control:Remote $(p<0.05)$. 

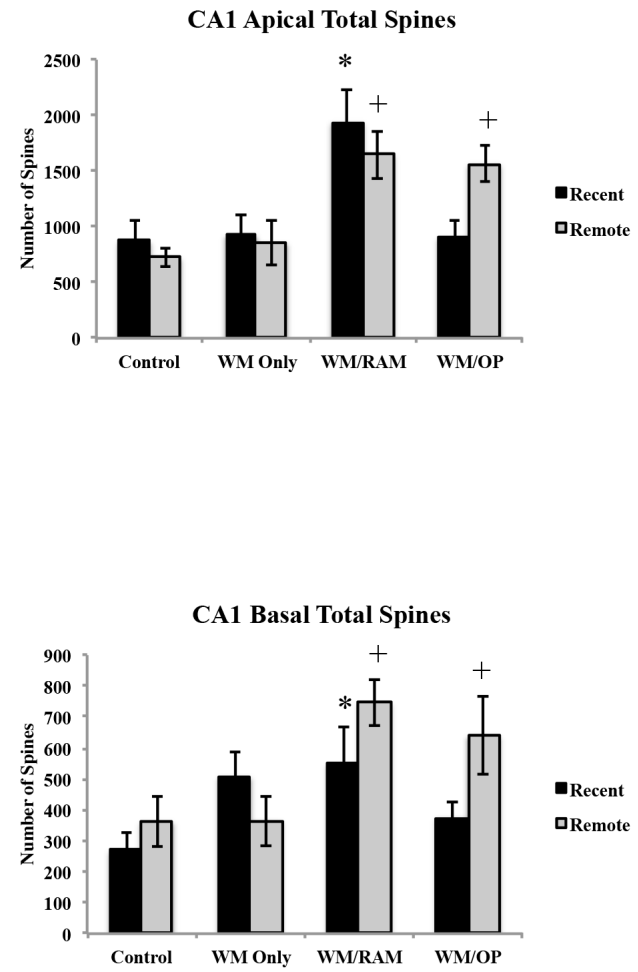

Figure 3.11: Total number of spines in the CA1 in apical and basal dendrites. Only significant differences between behavioural groups and their respective control group are displayed. Refer to Results section for all comparison results. * Significantly different from Control:recent group $(p<0.05)$; + Significantly different from Control:Remote $(p<0.05)$. 

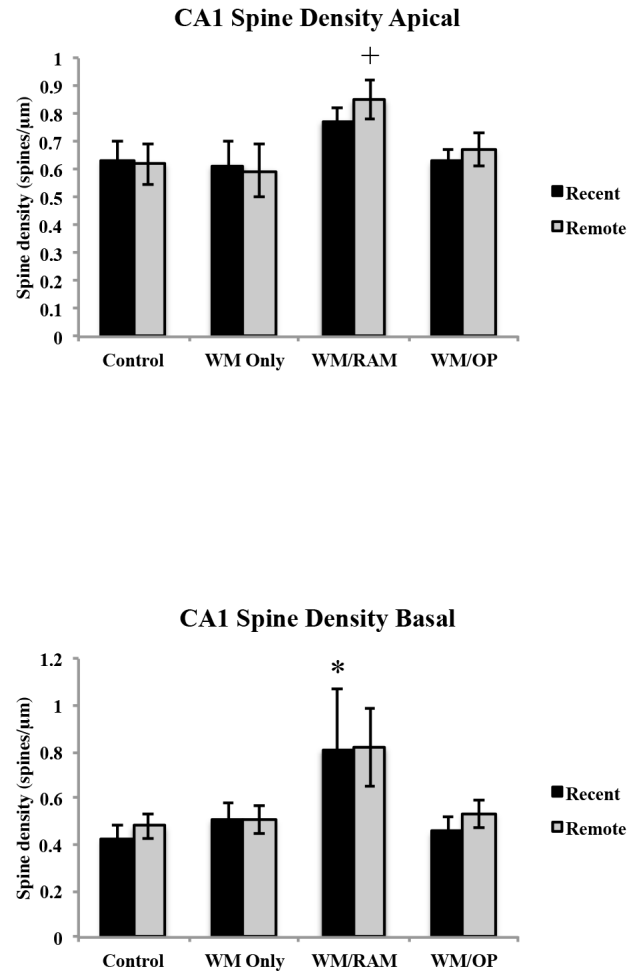

Figure 3.12: Spine density in the CA1 in apical and basal dendrites. Only significant differences between behavioural groups and their respective control group are displayed. Refer to Results section for all comparison results. * Significantly different from Control:recent group $(p<0.05)$; + Significantly different from Control:Remote $(p<0.05)$. 


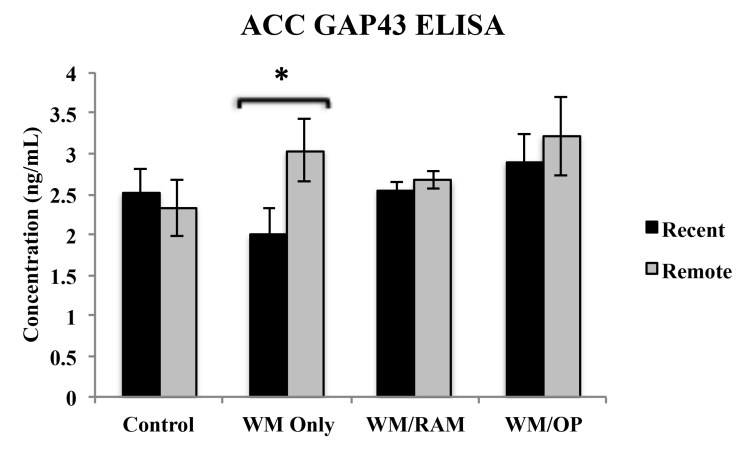

Figure 3.13: Concentration of GAP-43 in ACC tissue. * Indicates $p<0.05$ 


\section{Manuscript 3}

Manuscript 1 provided c-Fos results that suggested increasing the processing demands placed upon the hippocampus resulted in an accelerated involvement of the ACC in memory retrieval. Manuscript 2 revealed structural evidence that suggested increased processing demands on the hippocampus engaged synaptic and systems consolidation processes in the ACC at an accelerated rate. Manuscripts 1 and 2 suggested an involvement of the ACC in memory retrieval and storage, but did not determine its necessity in such processes. Therefore, the present study sought to determine if the ACC was a necessary structure involved in memory retrieval and whether the time course of necessity would be accelerated following training on multiple hippocampal-dependent tasks.

To this end, rats were trained on the multiple memory procedure as described in Manuscripts 1 and 2, however, pharmacological inactivation was used to transiently block ACC function. We assessed behavioural performance to infer whether deficits occurred as a result of ACC inactivation. 


\section{Chapter 4}

\section{Inactivation of the anterior}

cingulate reveals enhanced reliance on cortical networks for remote spatial memory retrieval after multiple memory processing

B.C. Wartman, J. Gabel \& M.R. Holahan

Submitted for publication to PLOS ONE 2014

Department of Neuroscience, Carleton University

Keywords: remote memory; hippocampus; anterior cingulate; spatial memory; water maze; radial arm maze; multiple memories 


\subsection{Abstract}

Systems consolidation models suggest that as time passes, ensembles of cortical neurons form strong connections to store and represent remote memories. While evidence points to the anterior cingulate cortex (ACC) as a cortical region that represents remote memories, there is debate as to whether remote spatial memories come to rely solely on the ACC for long-term storage. The present experiment sought to determine whether increasing the processing demand on the hippocampus, by sequential training on two spatial tasks, would more fully engage the ACC during retrieval of the remote spatial memory. In this scenario, inactivation of the ACC at a remote time point was hypothesized to produce a severe memory deficit if rats had been trained previously on two spatial tasks.

Rats were trained on a water maze (WM) task only or a WM task followed by a radial arm maze task. A WM probe test was given recently or remotely to all rats. Prior to the probe test, rats received an injection of saline or muscimol into the ACC. A subtle deficit in probe performance was found at the remote time point in the group trained on only one spatial task and treated with muscimol. In the group trained on two spatial tasks and treated with muscimol, a subtle deficit in probe performance was noted at the recent time point and a substantial deficit in probe performance was observed at the remote time point. c-Fos labeling in the hippocampus revealed more labeling in the CA1 region in all remotely tested groups than recently tested groups.

These results suggest continued involvement of the hippocampus in spatial memory retrieval along with a progressive strengthening of cortical connections as time progresses. Findings also suggest that spatial memories may come to rely more fully on the ACC when hippocampal processing requirements are increased. 


\subsection{Introduction}

Memory consolidation is a naturally occurring process whereby recently encoded memory representations become more resistant to decay over time. Consolidation processes apply to neural modifications that happen both at the cellular and systems levels (Dudai, 1996, 2000, 2004; Frankland and Bontempi, 2005, 2006; McGaugh et al., 2000; McGaugh, 2000). Systems consolidation is a gradual process, occurring over days to years, whereby memories may come to be represented by neural circuits that are linked to, but independent from, the neural ensembles that initially encoded and temporarily represented the memory. In this view, memory representations come to rely on distinct neural ensembles from those that initially encoded the memory for remote storage (Dudai, 2009; Frankland and Bontempi, 2005, 2006; Kandel, 2001; Kassardjian et al., 2005; Maviel et al., 2004; Wiltgen et al., 2004).

One brain structure that critically contributes to memory function is the hippocampus (Cohen and Eichenbaum, 1993; Scoville and Milner, 1957; Squire, 1992). Largely, though not exclusively, human research has shown that damage to the hippocampus results in temporally graded retrograde amnesia such that memories from the recent past are lost but memories from the remote past are spared (Bayley et al., 2006; Kapur and Brooks, 1999; Manns et al., 2003; Rosenbaum et al., 2000; Teng and Squire, 1999), which supports the hypothesis that memory representations encoded by the hippocampus become disengaged from those circuits over time for remote storage. Hippocampal damage in animal models has been reported by some to result in temporally graded retrograde amnesia (Ding et al., 2008; Kim and Fanselow, 1992; Maviel et al., 2004; Teixeira et al., 2006) but others have reported flat, or ungraded, retrograde amnesia following hippocampal damage (Broadbent et al., 2006; Clark et al., 2005a,b, 2007; Epp et al., 2008; Lehmann et al., 2007, 2013; Mumby et al., 1999; Sparks et al., 2011; Sutherland et al., 2008, 2001), such that memories from both the recent and remote past are disrupted.

One cortical region that may be involved in the storage of remote memories is the anterior cingulate cortex (ACC). Work studying the ACC at different time points after memory encoding has shown increased ACC activity on tests for remote memory ( $\sim 30$ days after encoding) (Bontempi et al., 1999; Frankland et al., 2004; Lopez et al., 2012; Maviel et al., 2004; Teixeira et al., 2006; Weible et al., 2012) and structural changes, indicative of memory storage, within the ACC have been observed at remote time points (Restivo et al., 2009; Vetere et al., 2011). In this framework, neural ensembles within the ACC would come to support memories for remote storage even in the absence of their involvement in initial memory encoding.

While a number of studies have shown that inactivation of the ACC hinders performance on remote memory tests (Ding et al., 2008; Frankland et al., 2004; Holahan et al., 2007; Lopez et al., 2012; Maviel et al., 2004; Teixeira et al., 2006) others have shown that inactivation of the ACC does not impair remote memory storage/retrieval (DeCoteau et al., 2009; Oswald et al., 2010). We ex-

plored this discrepancy by suggesting that a memory would more fully come to 
rely on neural ensembles in the ACC when distinct memories were sequentially encoded by overlapping neural ensembles in the hippocampus. Based on this, it was hypothesized that inactivation of the ACC would more fully disrupt remote memory retrieval in rats that had been trained on two sequential spatial tasks, the water and radial arm maze, as opposed to one. Both of these tasks rely on hippocampal neural ensembles for encoding so it might be the case that the ACC becomes fully engaged in memory storage when hippocampal demand is taxed during early phases of the consolidation process.

\subsection{Materials \& Methods}

\subsubsection{Subjects}

64 male Long Evans rats (190-250g) from Charles River, Quebec were used. Rats were housed individually in clear plastic cages $(26$ X 20 X $45 \mathrm{~cm})$ and given ad libitum water under a 12 hour light/dark cycle (lights on at 8:00 a.m.; rats tested during the light phase). Rats received no nesting material and no direct enrichment of any kind in their home cage. Rats acclimated to the vivarium environment for a minimum of five days prior to the beginning of experimental procedures. Principles of laboratory animal care were followed and all procedures were conducted in accordance with the Canadian Council on Animal Care and protocols approved by the Carleton University Animal Care Committee.

\subsubsection{Surgical Procedures}

Rats were administered Isofluorane gas anesthesia $\left(3 \%\right.$ in pure $\mathrm{O}_{2}$ to induce and $2 \%$ in pure $\mathrm{O}_{2}$ to maintain). Rats were defined to have reached an appropriate level of anesthesia when there was a loss of toe-pinch and pedal reflexes. This reflex was checked throughout surgical procedures. The head was shaved and rats were mounted in a stereotaxic apparatus. The scalp was cleaned and disinfected using betadine. Because the surgical procedures typically lasted approximately 45 minutes, a lubricating ophthalmic ointment (tear gel) was applied to the eyes of each rat. A midline incision in the scalp was made from behind the eyes to the ears using a $10 \mathrm{~mm}$ scalpel. Skin was scraped from the skull and hemostats were used to hold the incision open. Rats were bilaterally implanted with stainless steel $10 \mathrm{~mm}$ guide cannulas (25 gauge) into the anterior cingulate cortex (ACC). With the tooth bar set at $-3.9 \mathrm{~mm}$ (Paxinos and Watson, 2006) cannula tips were placed at coordinates (Anteroposterior: +1.2; Mediolateral: +/- 0.5; Dorsoventral: -2.0). Removable stylets (32 gauge) were inserted into the guide cannula to keep them free from blockage. The cannula were anchored to the skull with screws and dental cement. Polysporin was applied to the surgical area and the incision was closed using sutures. Topical lidocaine was applied to the incision site and a subcutaneous injection of Metacam $(0.1 \mathrm{~mL})$ was given. Rats were returned to their home cage, which was placed on a heating pad set to low. Soft food and water were placed inside the cage. Rats were under 
close surveillance until they were fully ambulatory. Basic biological functions including food and water intake, urination, defecation and body weight as well as clinical signs of distress (e.g., piloerection, reduced locomotion, hypothermia) were monitored daily. The surgical site and wound were monitored routinely for signs of infection. Rats recovered from surgery for a minimum of 7 days before further experimental procedures began.

\subsubsection{Apparatus}

\section{Water Maze (WM)}

The WM was located in a room within the animal housing area. The opaque, white, polypropylene pool measured $155 \mathrm{~cm}$ in diameter and $60 \mathrm{~cm}$ in height. The pool was filled to a depth of $37.5 \mathrm{~cm}$ with water that remained at approximately $21^{\circ} \mathrm{C}$. The escape platform was made from clear Plexiglas and submerged approximately $2 \mathrm{~cm}$ below the surface of the water. Visual cues such as posters and geometric shapes were located on the walls around the room. The experimenter remained in the same position throughout all trials.

\section{Radial Arm Maze (RAM)}

RAM testing was done in a room within the animal housing area, located across the hall from the WM testing room. The maze was positioned $98.5 \mathrm{~cm}$ off the floor. Each arm measured $59 \mathrm{~cm}$ long and $11 \mathrm{~cm}$ wide. The distance between the ends of arms, where food (chocolate pellet, BioServe, New Jersey) reward was located, was $32.5 \mathrm{~cm}$. Plastic inserts were placed on the sides of the maze arms to prevent animals from jumping across arms. Visual cues such as posters and geometric shapes were located on the walls around the room. The experimenter remained in the same position throughout all trials.

\subsubsection{Behavioural Procedure}

An overview of the behavioural procedure is shown in Figure 4.1. Seven days following surgery, rats were food restricted to $90 \%$ of their free feeding body weight over a minimum of 10 days. Rats were assigned to one of two behavioural groups: (1) Training on one spatial task, the WM; or (2) Training on two spatial tasks, the WM followed by the RAM. Sequential training on two tasks was separated by a 24 hour rest period. Following training, rats received either a recent WM probe test ( 8 days after the end of WM training, $\mathrm{n}=32$ ) or a remote WM probe test (37 days after the end of WM training, $\mathrm{n}=32$ ). Additionally, 15 minutes prior to the probe test, rats were assigned to receive an intracranial injection of muscimol, a $\mathrm{GABA}_{A}$ receptor agonist, or saline. Eight experimental groups resulted ( $\mathrm{n}=8$ in each group): (1) WM:Recent:Saline (2) WM:Recent:Muscimol (3) WM/RAM:Recent:Saline (4) WM/RAM:Recent:Muscimol (5) WM:Remote:Saline (6)WM:Remote:Muscimol (7) WM/RAM:Remote:Saline (8) WM/RAM:Remote:Muscimol. 


\section{Water Maze}

Rats received 5 training trials per day for 5 days, with a different starting location for every trial within a day and randomized starting locations across days. The hidden platform was located in a fixed location within and across days. Rats were placed in the pool, facing the perimeter, and given a maximum of 60 seconds to locate the hidden platform. Rats that did not find the hidden platform within 60 seconds were guided to the platform by the experimenter. All rats remained on the platform for 15 seconds. Rats then received a 15 second rest period in a holding cage before the next trial. All movement within the pool was tracked using HVS Image 2100 Tracking System (HVS Image, Buckingham, UK). Following the final trial of each day, rats were dried with a towel and placed in a holding cage on a heating pad in the housing room for 10-15 minutes after which they were returned to the home cage.

\section{Radial Arm Maze}

Rats received one day of pretraining and 4 days of testing on the RAM. On the first trial of pretraining, chocolate pellets were located in the starting area, at the entrance to arms, within the arms as well as in the food holes located at the end of each arm. On trial 2 of pretraining, pellets were located within the arms and in food holes only. Trials 3-5 of pretraining had pellets located only in food holes.

Days 2-5 were testing days, where pellets were located in food holes at the ends of 3 of the 5 arms. Baited arms were always the same for an individual rat but differed between rats. Each rat was given 5 trials per day. Trials were a maximum of 5 minutes each or ended when all food reward had been collected. Rats were placed in a holding cage for 30 seconds between trials while arms were re-baited. Performance on the maze was manually scored. Sessions were timed, and correct and incorrect arm entries were recorded. An arm entry was defined as all four feet inside an arm.

\subsubsection{Injections and Water Maze Probe}

Rats were handled daily throughout experimental procedures and rest periods to minimize stress during testing and injection procedures. Injections were performed on awake rats, held by the experimenter for the duration. Stylets were removed and two stainless-steel injection cannula (32 gauge), connected to two individual $10 \mu \mathrm{L}$ Hamilton syringes via polyethylene tubing, were carefully lowered through the guide cannula. The syringes were connected to an injection pump (Braintree Scientific, Inc.). The pump was programmed to deliver a volume of $0.5 \mu \mathrm{L}$ of muscimol $(50 \mathrm{ng} / 0.5 \mu \mathrm{L})$ or saline solution at a rate of $0.25 \mu \mathrm{L} /$ minute, with a total infusion time of two minutes. The injection cannula were left in place for an additional one minute. The injectors were removed and rats were placed in their home cage for 15 minutes before probe testing.

The escape platform was removed from the water maze pool for the probe 
test. Rats were placed in the pool and free to swim for 60 seconds. Rats were then removed from the pool, dried with a towel, placed on a heating pad and brought back to the home cage room. Sixty minutes after the end of the WM probe test, rats were placed into a Decapicone (Braintree) and decapitated. Brains were rapidly removed and hemisected (hemispheres were counterbalanced). One hemisphere was placed in $4 \%$ paraformaldehyde/0.01M phosphate buffer solution ( $\mathrm{PB} ; \mathrm{pH} 7.4)$ overnight at $4^{\circ} \mathrm{C}$. The following day brains were cryoprotected in a $30 \%$ sucrose $/ 0.01 \mathrm{M} \mathrm{PB}$ solution and stored at $4^{\circ} \mathrm{C}$ for a minimum of 72 hours or until sectioned.

\subsubsection{Tissue Processing}

\section{Cresyl Violet}

Brains were sectioned at $30 \mu \mathrm{m}$ on a Leica CM1900 cryostat (Weztler, Germany). Sections were stored in a $0.1 \%$ sodium azide/ $0.01 \mathrm{M}$ PB solution at $4^{\circ} \mathrm{C}$. Sections containing the ACC were float mounted on microscope slides and placed in $100 \%, 95 \%$, and $70 \%$ ethanol solutions for two minutes each. Excess ethanol was removed with a rinse in distilled water. Sections were then placed in a $1 \%$ Cresyl Violet solution for three minutes. Excess stain was removed with a rinse in distilled water. Sections were place in a $0.8 \%$ acetic acid solution until fiber tracks became unstained (approximately 3-5 minutes). Sections were placed in $70 \%, 95 \%$ and $100 \%$ ethanol solutions for two minutes, followed by a 15 minute incubation in Clearene. Slides were coverslipped using Permount mounting medium. Once dry, sections were examined under a light microscope to verify cannula placements. See Figure 4.7.

\section{c-Fos}

Sections were washed for $3 \mathrm{X} 5 \mathrm{~min}$ in a $0.2 \%$ Triton-X/0.01 M phosphatebuffered saline (T-PBS) solution then blocked for 15 minutes in a $0.3 \% \mathrm{H}_{2} \mathrm{O}_{2}$ T-PBS solution. Sections were washed for 3 X 5 min in T-PBS solution then blocked in a 3\% Animal Free Blocker (AFB) (Vector)/ T-PBS solution for 30 minutes followed by incubation in the primary antibody (rabbit anti-c-Fos from Abcam, 1:5000) overnight at room temperature. The following day, sections were washed for 3 X $10 \mathrm{~min}$ in T-PBS solution then incubated for two hours in the secondary antibody (biotinylated goat anti-rabbit, 1:500). Sections were then washed 3 X 10 min in T-PBS followed by one-hour incubation in an avidinbiotinylated complex (ABC Elite kit; Vector Laboratories). Sections were given a wash in PBS then reacted with a $0.25 \%$ 3,3'-diaminobenzedine tetrahydrochloride (DAB) solution in PBS solution containing $0.0025 \% \mathrm{H}_{2} \mathrm{O}_{2}$ for 6 minutes. All sections were given a final rinse in $0.01 \mathrm{M}$ PBS for 15 minutes and mounted on glass slides. Sections were dehydrated and coverslipped with glass coverslips and Permount (Sigma). 


\subsection{7 c-Fos quantification}

Utilizing unbiased stereological principles, the Optical Fractionator method provided an estimate of the number of c-Fos positive cells in the CA1 region of the dorsal hippocampus. Stained sections were visualized using an Olympus BX51 brightfield microscope with a motorized stage (Olympus Canada, Markham, $\mathrm{ON}$ ) and images captures with an Olympus U-CMAD3 camera. Stereo Investigator (MBF Bioscience, Williston, VT) software was used for unbiased stereological quantification. The CA1 volume of interest focused on the anterior aspect of the dorsal hippocampus and was restricted in the anterior-posterior plane from bregma -3.3 to $-3.6 \mathrm{~mm}$. The region of interest for each section was traced digitally at $4 \mathrm{X}$ magnification with reference to (Paxinos and Watson, 2006). Two to three random coronal sections were sampled from this region from each rat. c-Fos counting was performed using sampling parameters sufficient to produce a Gundersons coefficient of error $(\mathrm{GCE}, \mathrm{m}=1)$ less than 0.1, which has been established to be a suitable coefficient of error estimate (Blum et al., 2012; Gundersen and Jensen, 1987; Gundersen et al., 1999). A minimum of 6 animals per group were included in the analysis. Counting parameters were set to a counting frame of $30 \mathrm{X} 30 \mu \mathrm{m}^{2}$ and a dissector height of $10 \mu \mathrm{m}$ between the top and bottom guard zones. c-Fos positive cells were quantified using a $60 \mathrm{X}$ magnification lens (oil immersion, NA 1.35) when the uppermost tip of c-Fos positive nuclei were in focus within the counting frame and the dissector height. Stereo Investigator software used planar and depth information for each counted nuclei to calculate the volume for the digitally traced region of interest. Quantification is represented as an estimated total per mean measured thickness per $10,000 \mu \mathrm{m}^{3}$ to allow for comparisons across brain sections.

\subsection{Results}

\subsubsection{Behavioural Training}

An overview of the behavioural procedure is shown in Figure 4.1. Rats were randomly assigned to one of two behavioural groups: (1) Training on one spatial task, the WM or (2) Training on two spatial tasks, the WM followed by the RAM. Sequential training on two tasks was separated by a 24 hour rest period. Following training, rats received either a recent WM probe test ( 8 days after the end of WM training, $\mathrm{n}=32$ ) or a remote WM probe test (37 days after the end of WM training, $\mathrm{n}=32$ ). Fifteen minutes prior to the probe test, rats were assigned to receive an intracranial injection of muscimol, a $\mathrm{GABA}_{A}$ receptor agonist, or saline into the ACC. Eight experimental groups resulted ( $\mathrm{n}=8 /$ group): (1) WM:Recent:Saline (2) WM:Recent:Muscimol (3) WM/RAM:Recent:Saline (4) WM/RAM:Recent:Muscimol (5) WM:Remote:Saline (6)WM:Remote: Muscimol (7) WM/RAM:Remote:Saline (8) WM/RAM:Remote:Muscimol.

Figure 4.2 shows acquisition data for both the WM and RAM tasks. All rats received WM training. Latency to reach the hidden platform was recorded (Figure 4.2A). To reduce visual noise, recent and remote time points were plot- 
ted and examined separately. At the recent time point, a repeated measures ANOVA with day (1 to 5) as the within-subject factor and behaviour (WM Only or WM/RAM) and treatment (Saline or Muscimol) as the between-subjects factors revealed a main effect of day $\left(\mathrm{F}_{(4,112)}=73.14, p<0.001\right)$ but no main effect of behaviour or treatment. At the remote time point, a repeated measures ANOVA with day (1 to 5) as the within-subject factor and behaviour (WM Only or WM/RAM) and treatment (Saline or Muscimol) as the between-subjects factors revealed a main effect of day $\left(\mathrm{F}_{(4,112)}=140.28, p<0.001\right)$ but no main effect of behaviour or treatment.

Four groups received training on the WM followed by training on the RAM: one assigned to receive a recent WM probe test (Saline or Muscimol) and one assigned to receive a remote WM probe (Saline or Muscimol) (Figure 4.2B). The number of errors (unbaited arm entries and re-entries) made during training was analyzed with a repeated measures ANOVA with day (2 to 4) as the withinsubject factor and time (recent or remote) and treatment (saline or muscimol) as the between-subject factors. Analyses revealed a main effect of day $\left(\mathrm{F}_{(3,84)}=\right.$ $7.85, p<0.001$ ) but no main effect of time or treatment.

\subsubsection{Water Maze Probe Test}

To provide an accurate depiction of the search patterns exhibited by the different groups during the probe test, data were examined for the entire 60 second probe test and also parsed into two time bins: from 0-30 seconds and 31-60 seconds. This analysis allowed us to examine whether the initial search patterns (030 seconds) focused on the correct area of the pool while later search patterns (31-60 seconds) expanded to other areas of the pool or remained in the correct (target) area of the pool.

\section{Surface Plot}

Average dwell times in discrete regions of the water maze for the eight groups over the 60 second water maze probe test are illustrated in Figure 4.3 as surface or occupancy plots generated from Wintrack software (Wolfer et al., 2001). Visually, the surface plots show a clear preference in search strategy for the area associated with the platform location (circle) in groups WM Only:Recent:Saline, WM Only:Recent:Muscimol, WM Only:Remote:Saline WM/RAM:Recent Saline, and WM/RAM:Remote Saline. Groups WM Only:Remote:Muscimol and WM/ RAM:Recent:Muscimol showed less accurate search patterns for the area associated with the platform location. A clear reduction in dwell time in the platform location can be seen in group WM/RAM:Remote:Muscimol.

\section{Target Quadrant}

Rats received either a recent or remote WM probe test. The amount of time spent swimming in the target quadrant (quadrant where the platform was located during training) was examined. Separate 2 X 2 X 2 (behaviour (WM Only 
or WM/RAM) X time (recent or remote) X treatment (saline or muscimol) fixed factor ANOVAs were run.

Statistical analyses on time spent in the target quadrant during the 60 second probe (Figure 4.4A) revealed no main effect of behaviour, time or treatment $(p>0.05)$. A significant behaviour $\mathrm{X}$ injection interaction was found $\left(\mathrm{F}_{(1,56)}=\right.$ $4.48, p<0.05)$. When time spent searching in the target quadrant was compared to chance $(25 \%)$, all experimental groups spent above chance amounts of time searching in the target quadrant except group WM/RAM:Remote:Muscimol.

Statistical analyses on time spent in the target quadrant from $0-30$ seconds (Figure 4.4B) revealed a main effect of time $\left(\mathrm{F}_{(1,56)}=5.40, p=0.02\right)$ with recent groups spending greater amounts of time in the target quadrant compared to remote groups (Recent mean: $49.90 \%$ Remote mean: $40.53 \%$ ). A significant interaction between behaviour and treatment $\left(\mathrm{F}_{(1,56)}=3.73, p=0.05\right)$, and a significant three-way interaction between behaviour, time and treatment $\left(\mathrm{F}_{(1,56)}=4.82, p=0.03\right)$ were found. When time spent searching in the target quadrant was compared to chance (25\%), all experimental groups spent above chance amounts of time searching in the target quadrant during the first 30 seconds of the probe test except group WM/RAM:Remote:Muscimol.

Statistical analyses on time spent in the target quadrant from 31-60 seconds (Figure 4.4C) revealed fewer groups spent above chance amounts of time searching in the target quadrant (WM Only:Recent:Saline, WM Only:Recent:Muscimol, WM Only:Remote:Saline, WM Only:Remote:Muscimol and WM/RAM:Remote: Saline spent above chance amounts of time searching in the target quadrant). A significant interaction between behaviour and time $\left(\mathrm{F}_{(1,56)}=4.35, p=0.04\right)$ was found, but no other significant effects or interactions were noted.

\section{Target Counter Region}

The amount of time spent swimming in the target area (2X the diameter of the platform; referred to as counter in the parlance of the HVS Image software) was examined. Separate 2 X 2 X 2 (behaviour (WM Only or WM/RAM) X time (recent or remote) $\mathrm{X}$ treatment (saline or muscimol)) fixed factor ANOVAs were run.

Statistical analyses on time spent in the target area during the 60 second probe (Figure 4.5A) revealed a main effect of time $\left(\mathrm{F}_{(1,56)}=5.27, p<0.05\right)$, with recently probed rats spending greater amounts of time in the target area $(7.05 \%)$ compared to remotely probed rats $(4.81 \%)$. When time spent searching in the target area was compared to chance $(2 \%)$, experimental groups WM Only:Recent:Saline, WM Only:Recent:Muscimol, WM/RAM:Recent:Saline, WM Only:Remote:Saline and WM/RAM:Remote:Saline spent above chance amounts of time searching in the target area $(p<0.05)$. Groups WM/RAM:Recent: Muscimol, WM: Only:Remote:Muscimol and WM/RAM:Remote:Muscimol did not spent above chance amounts of time searching in the target area $(p<0.05)$.

Statistical analyses on time spent in the target area from 0-30 seconds (Figure $4.5 \mathrm{~B})$ revealed a main effect of time $\left(\mathrm{F}_{(1,56)}=11.82, p<0.001\right)$ with recently probed rats spending a greater amount of time in the target area com- 
pared to remotely probed rats (Recent mean: 9.91\% Remote mean: 5.44\%). A significant three-way interaction between behaviour, time and treatment $\left(\mathrm{F}_{(1,56)}=3.77, p=0.05\right)$ was found. Fishers LSD post hoc tests revealed group WM/RAM:Remote:Muscimol spent less time swimming in the target area compared to all other groups (except WM Only:Remote:Saline $(p=0.11)$ and WM Only:Remote: Muscimol $(p=0.08)$ ). When the time spent searching in the target area was compared to chance $(2 \%)$ all experimental groups spent above chance amounts of time in the target area during the first 30 seconds of the probe test except group WM/RAM:Remote:Muscimol.

Statistical analyses on time spent in the target counter region from 31-60 seconds (Figure 4.5C) revealed only group WM/RAM:Remote:Saline spent above chance amounts of time searching in the target area. No other main effects or significant differences between experimental groups were found.

\section{Target Area Crossings}

The number of crosses through the target area (2X the diameter of the platform) was examined. Separate 2 X 2 X 2 (behaviour (WM Only or WM/RAM) X time (recent or remote) $\mathrm{X}$ treatment (saline or muscimol)) fixed factor ANOVAs were run.

Statistical analyses on the number of crossings during the 60 second probe (Figure 4.6A) revealed a main effect of time $\left(\mathrm{F}_{(1,56)}=7.57, p<0.01\right)$ with recently tested rats having a greater number of crosses through the target area (5.25) compared to remotely tested rats (3.56).

Statistical analyses on the number of crossings from 0-30 seconds (Figure 4.6B) revealed a main effect of time $\left(\mathrm{F}_{(1,56)}=18.88, p<0.001\right)$ with recently probed rats displaying a greater number of crossings through the target area (Recent mean: 3.41 Remote mean: 1.91). A significant three-way interaction between behaviour, time and treatment $\left(\mathrm{F}_{(1,56)}=6.43, p<0.02\right)$ was found. Fishers LSD post hoc tests revealed group WM/RAM:Remote:Muscimol displayed fewer crossings through the target area during the first 30 seconds of the probe test compared to all other groups $(p=0.05)$.

Statistical analyses on the number of crossings through the target area from 31-60 seconds (Figure 4.6C) revealed no main effects or significant differences between experimental groups.

\subsection{3 c-Fos labeling in the CA1 of the hippocampus}

Figure 4.7A shows quantification of the number of c-Fos positive cells per $10,000 \mu \mathrm{m}^{3}$ in the CA1 of the hippocampus. Figure 4.7B (Paxinos and Watson, 2006) depicts the CA1 region of the hippocampus where c-Fos positive cells were counted. Figure $4.7 \mathrm{C}$ displays representative images of c-Fos positive neurons at 4X, 20X and 60X magnification. A 2 X 2 X 2 (behaviour (WM Only or WM/RAM) X time (recent or remote) $\mathrm{X}$ treatment (saline or muscimol) fixed factor ANOVA revealed a main effect of time $\left(\mathrm{F}_{(1,49)}=16.509, p<0.001\right)$ with remotely probed rats showing greater numbers of c-Fos positive cells compared 
to recently probed rats (Recent mean: 3.279 Remote mean: 4.157).

\subsubsection{Placements}

Figure 4.8 shows representative placements of injector tips aimed at the ACC. Postmortem analyses of cannula and injector placements confirmed consistent placement of injections into the ACC of all rats used. The range in injector tip locations was: Anteroposterior: +1.7 to +0.7 ; Mediolateral: $+/-0.2$ to 0.7 ; Dorsoventral: -2.0 to -3.0 . This range is well within the boundary of the ACC and, given prior estimates of the volume of inactivation produced by a similar volume and dose of muscimol (Holahan and White, 2004; Wang and Redgrave, 1997), the entire ACC was likely inactivated by the muscimol injections.

\subsection{Discussion}

The purpose of this study was to investigate whether sequential training on two spatial tasks would more fully engage the ACC during retrieval of a remote spatial memory. Inactivation of the ACC at a remote time point was hypothesized to produce a severe memory deficit if rats had been trained on two spatial tasks. Results revealed a substantial impairment in remote spatial memory recall in the group trained on two spatial tasks and injected with muscimol into the ACC prior to the remote probe test (WM/RAM:Remote:Muscimol). This group failed to show evidence of a memory for the platform location by spending chance amounts of time in the target quadrant and target area. Additionally, this group made fewer crossings through the target area compared to all other experimental groups. The control condition for this group (WM/RAM:Remote:Saline) showed a strong memory for the platform location during the probe test on all indices analyzed, emphasizing the profound memory deficit seen in group WM/RAM:Remote:Muscimol. The severely impaired performance in group WM/RAM:Remote:Muscimol supports the hypothesis that a remote spatial memory comes to rely more fully on neural ensembles in the ACC when distinct memories are sequentially encoded by overlapping neural ensembles in the hippocampus.

A slight deficit in memory recall was seen in groups WM Only:Remote: Muscimol and WM/RAM:Recent:Muscimol. The overall performance on the probe test revealed that these groups did not spend above chance amounts of time searching in the target area, indicative of a memory retrieval deficit. More stringent behavioural analyses revealed that these groups displayed above chance amounts of time in the target area during the first 30 seconds of the probe test, but dropped to chance amounts of time in the target area during the last 30 seconds of the probe test. The moderate impairment in group WM Only:Remote:Muscimol is in line with past research which has shown that the ACC becomes engaged in remote memory recall (Ding et al., 2008; Frankland et al., 2004; Holahan et al., 2007; Maviel et al., 2004; Teixeira et al., 2006). The slight impairment in group WM/RAM:Recent:Muscimol is consistent with 
recently published research which suggests that taxing the demand placed upon the hippocampus, by training rats on two sequential spatial tasks, engages the recruitment of the ACC at earlier time points (Wartman and Holahan, 2013).

The pattern of impairments seen in this study supports the idea of a progressive strengthening of cortical connections over time, as suggested by the standard consolidation model (Frankland and Bontempi, 2005). The group trained on a single hippocampal-dependent task (WM Only) did not show evidence of reliance upon the ACC at the recent time point as there was no deficit in performance following muscimol treatment. Over time, the memory representation in the WM Only remote group came to partially rely on the ACC, as a slight deficit in probe performance was seen in this group following muscimol treatment. These results suggest the remote memory for the WM task in the WM only group was not initially dependent upon the ACC but as time progressed, the memory may have become represented in neural ensembles in both the hippocampus and ACC. One factor that may be important in enhancing the dependence of a remote memory representation on ACC networks is the processing demand placed on the hippocampus during early stages of consolidation (Wartman and Holahan, 2013). In line with this, results from the present study showed a substantial impairment in the group trained on the two spatial tasks and injected with muscimol into the ACC prior to the remote time point. These results support the hypothesis of a progressive strengthening of cortical connections as time progresses and an accelerated strengthening of cortical networks with increasing memory processing demands.

It should be emphasized that group WM/RAM:Remote:Muscimol showed no indication of an intact memory representation for the platform location. Multiple memory traces that are hippocampal-dependent, such as those arising from spatial tasks, may compete for hippocampal resources if they are encoded within a relatively short timeframe. In this situation, reliance upon cortical structures, such as the ACC, may more readily occur so that subsequent memory traces do not interfere with one another. Transferring the primary memory representation from the hippocampus to cortical regions may free hippocampal resources to process and encode a new memory trace. As many models suggest (Frankland and Bontempi, 2005; Roxin and Fusi, 2013; Squire and Alvarez, 1995) a gradual shift in memory storage from the hippocampus to cortical regions is likely and advantageous. However, multiple memories relying heavily on hippocampal resources may accelerate the time course of this shift in an effort to free hippocampal resources. Future studies could manipulate the basic behavioural procedure outlined in this experiment to test the robustness of this hypothesis. For example, the order of training tasks could be reversed, or a RAM probe (instead of a WM probe) could be used. It would also be of interest to conduct the present experiment using a non-spatial task following WM training.

To accompany the behavioural findings, we examined c-Fos labeling patterns in the CA1 region of the hippocampus. Stereological analyses revealed remotely tested groups had significantly higher numbers of c-Fos positive cells than recently tested groups. This finding contradicts some earlier reports that revealed lower hippocampal activation at remote time points (Frankland et al., 
2004; Maviel et al., 2004) but is similar to other reported data (Bonaccorsi et al., 2013; Lopez et al., 2012; Schlesiger et al., 2013; Wartman and Holahan, 2013). As the WM task is a hippocampal-dependent task, it comes as no surprise the hippocampus was activated during the probe test (Broadbent et al., 2006; Clark et al., 2005a,b; D'Hooge and De Deyn, 2001; Holahan and Routtenberg, 2011; Morris et al., 1986, 1982; Ramos, 2009; Remondes and Schuman, 2004; Sutherland et al., 2001, 1983; Teixeira et al., 2006; Wiltgen et al., 2010). From the current study, it is impossible to tell whether the increase in c-Fos positive cells resulted from increased activation of the hippocampus due to performance of the task (i.e., swimming), or, whether the increase resulted from a cognitive process (i.e., retrieving the stored memory).

Discrepancies in c-Fos labeling in the hippocampus may result from different behavioural tasks used in assessment. Bonaccorsi et al. (2013), Wartman and Holahan (2013), and Lopez et al. (2012), used the water maze task as a behavioural measure and found increased activation of the hippocampus at remote compared to recent time points, consistent with the present set of results. Schlesiger et al. (2013) used a modified radial arm maze task that incorporated different start locations and retention testing in a single trial in an effort to incorporate aspects of a standard water maze procedure. Frankland et al. (2004) used contextual fear conditioning, while Maviel et al. (2004) used a five-arm maze as their behavioural measure. Other studies have revealed similar Fos expression following recent and remote probes on the water maze task (Teixeira et al., 2006). Factors surrounding the task used such as complexity, cue saliency, and other environmental influences may result in a greater burden placed upon the hippocampus during remote memory retrieval.

Interestingly, group WM/RAM:Remote:Muscimol (as well as WM Only: Remote:Muscimol and WM/RAM:Remote:Muscimol), similar to all other remotely probed groups, showed greater numbers of c-Fos positive cells than recently probed muscimol or saline groups, but showed impaired performance on the water maze probe test when the ACC was inactivated. As one interpretation of our findings (elevated c-Fos labeling in the CA1 in all remote groups, but no consistent behavioural evidence for an intact memory representation at the remote time point), we suggest that elevated activity in hippocampal networks is required for retrieving a remote spatial memory and that remote spatial memory is supported in networks in the ACC. Alternatively, it could be the case that the ACC is involved in remote memory retrieval from the hippocampus, and when offline, cannot perform this function. This interpretation would require an experimental design that allows for the testing of this hypothesis.

The findings presented here suggest continued involvement of the hippocampus in memory retrieval as well as involvement of the ACC. The pattern of behavioural findings suggests a progressive strengthening of cortical connections as time progresses and an accelerated involvement of the ACC in memory representation when hippocampal demand is taxed. 


\section{Supplemental material not included in Manuscript 3}

\subsection{Supplemental Introduction}

Protein kinase $\mathrm{M} \zeta(\mathrm{PKM} \zeta)$ is a constitutively active $\mathrm{PKC}$ isoform that lacks the regulatory domain of PKC and contains only the catalytic domain (Hernandez et al., 2003). As a second-messenger independent kinase, its activity persists longer than usual for a typical kinase. PKM $\zeta$ plays a seemingly critical role in the maintenance of L-LTP as blocking the activity of $\mathrm{PKM} \zeta$ results in the reversal of late-phase LTP, but does not disrupt early-phase LTP (Pastalkova et al., 2006; Serrano et al., 2005). $\operatorname{PKM~} \zeta$ has been hypothesized to be involved in forming and preserving structural changes at synapses thought to be critical for maintaining long-term memories (Serrano et al., 2005; Shao et al., 2011; Shema et al., 2007). Locally inhibiting $\operatorname{PKM} \zeta$ days, weeks or months after training can erase long-term memories, both declarative and procedural, illustrating its profound and potent effect on memory storage (Madroñal et al., 2010; Migues et al., 2010; Shema et al., 2007; von Kraus et al., 2010). PKM s suspected mode of action is through the maintenance of glutamate receptor 2 (GluR2) at the postsynaptic density. Evidence suggests $\operatorname{PKM} \zeta$ blocks a pathway important in removing certain postsynaptic AMPAR, thereby increasing levels of postsynaptic AMPAR persistently (Hernandez et al., 2003; Ling et al., 2006, 2002; Migues et al., 2010; Sacktor, 2008; Shao et al., 2011; Yao et al., 2008).

In neurons, $\operatorname{PKM} \zeta$ mRNA is produced from an internal promoter within the PKC $\zeta$ gene, which produces a $\zeta$ catalytic domain lacking a regulatory domain. $\mathrm{PKM} \zeta$ mRNA is shuttled to the dendrites of neurons but is translationally blocked until signalling cascades set off by LTP induction (such as CaMKII, PKA, and NMDAR activation) release the block. Following translation, PKM $\zeta$ binds to and is phosphorylated by phosphoinositide-dependent protein kinase 1 (PDK1), which increases $\mathrm{PKM} \zeta$ s constitutive activity, and maintains increased translation of itself. PKM $\zeta$ regulates AMPAR trafficking by effecting the interaction between N-ethylmaleimide-sensitive factor (NSF) and GluR2, a relationship which, under basal conditions keeps a homeostatic balance of AMPARs to potentiate synaptic transmission by increasing the number of GluR2-containing subunits at postsynaptic sites (Migues et al., 2010; Sacktor, 2008, 2011; Yao et al., 2008).In addition to its effect on AMPAR trafficking, PKM $\zeta$ may have effects on neuronal structure. Ron et al. (2012) demonstrated that overexpression of $\mathrm{PKM} \zeta$ increases the proportion of mature spines (stubby and mushroom shaped spines) in cortical neurons. This corresponded to an increase in the amplitude of spontaneous miniature excitatory synaptic potentials, believed to reflect an increase in synaptic strength. PKM $\zeta$ overexpression in hippocampal neurons increased the size of dendritic spines and PSD-95 clusters, suggesting structural effects mediated by $\mathrm{PKM} \zeta$.

To provide a more detailed profile of protein expression, we examined PKM $\zeta$ protein following recent and remote WM probe tests based on behavioural manipulations from Manuscripts 1 and 3. 


\subsection{Supplemental Materials \& Methods}

Subjects and behavioural procedures were the same as outlined in Manuscript 1 and 3.

\subsubsection{Western Blot}

Rats were decapitated, brains were rapidly removed, hemisected and flash frozen.

\section{Sample preparation}

The ACC and HPC were dissected from the brain. Samples were prepared using Radio Immuno-Precipitation Assay (RIPA) lysis buffer, phosphatase inhibitor and protease inhibitor (Sigma). A mechanical pestle was used to homogenize tissue. Samples were spun at $5000 \mathrm{Xg}$ for 5 minutes. The supernatant was removed and placed into a fresh tub and the pellet was discarded. To determine protein concentration in the samples, a Pierce BCA Protein Assay Reagent kit was used (Thermo Scientific). $10 \mu \mathrm{g}$ samples were prepared for loading with Laemmli buffer and $5 \%$ beta mercaptoenthanol (BME).

\section{Protein separation and transfer}

$7.5 \%$ separating gels and $4 \%$ stacking gels were cast. Samples were heated at $95^{\circ} \mathrm{C}$, spun, cooled and loaded into the gel. The gel was run at 140 volts for 40 minutes. The gel was transferred to PVDF membranes using semi-dry transfer at 15 volts for 17 minutes. Membranes were blocked in $5 \%$ milk overnight.

\section{Immunoblot and development}

The primary antibody used for western blotting was a polyclonal rabbit antiPKC $\zeta$ derived from the middle region of the catalytic domain. This antibody reacts with $\mathrm{PKM} \zeta$ proteins in the brain with a band at approximately $55 \mathrm{kDa}$. Glyceraldehyde 3-phosphate dehydrogenase (GAPDH) is considered a housekeeping protein and is constitutively expressed at high levels in neurons making it a suitable choice as a loading control for Western blot.

Membranes were incubated in primary antibody (PKC $\zeta$ (Zymed, Life Technologies) 1:300; GAPDH (Ambion, Life Technologies) 1:10000) for one hour at room temperature. Membranes were rinsed 3X10 minutes in Tris Buffered Saline and Tween 20 (TBST). Membranes were incubated in secondary antibody (anti rabbit anti mouse) at 1:20000 for one hour at room temperature. Membranes were rinsed 3X10 minutes in TBST. Membranes were incubated in chemiluminesence (Bio-Rad) for approximately 3 minutes. Following chemiluminesence, membranes were developed on film in a dark room. 


\section{Quantification}

Alpha Ease (Protein Simple) software was used to quantify Western blot results. Developed film was scanned into a computer as a gray scale image. Alpha Ease analysis Spot Denso software was used to provide an integrated density value (IDV). IDV of the protein of interest $(\mathrm{PKC} \zeta)$ was divided by the IDV of the control protein (GAPDH) to obtain an IDV ratio.

\subsection{Supplemental Results}

Figure 4.9 shows a representative blot depicting bands representing both $\mathrm{PKC} \zeta$ (approx. 55kDa) and GAPDH (approx. 37kDa). Quantification of the ratio of PKC $\zeta$ IDV:GAPDH IDV is displayed in Figure 4.10. In tissue from rats that underwent behavioural procedures from Manuscript 1, separate 3X2 (Behaviour $\mathrm{X}$ Time) ANOVAs were run on ACC and hippocampal tissue. No main effect of behaviour $\left(\mathrm{F}_{(2,44)}=1.37, p<0.05\right)$, time $\left(\mathrm{F}_{(1,44)}=0, p<0.05\right)$, and no significant interaction $\left(\mathrm{F}_{(2,44)}=0.37, p<0.05\right)$ between the two was found in the ACC. Similarly, no main effect of behaviour $\left(\mathrm{F}_{(2,46)}=1.4, p<0.05\right)$, time $\left(\mathrm{F}_{(1,46)}=\right.$ $0.07, p<0.05)$, and no significant interaction $\left(\mathrm{F}_{(2,46)}=0.09, p<0.05\right)$ between the two was found in the hippocampus.

In tissue from rats that underwent surgical and behavioural procedures from Manuscript 3, a 2X2X2 (Behaviour X Time X Treatment) ANOVA run on hippocampal tissue revealed no main effect of behaviour $\left(\mathrm{F}_{(1,44)}=1.5, p<0.05\right)$, time $\left(\mathrm{F}_{(1,44)}=2.14, p<0.05\right)$ or treatment $\left(\mathrm{F}_{(1,44)}=0.1, p<0.05\right)$. No significant interactions were found (behaviour $\mathrm{X}$ time, behaviour $\mathrm{X}$ treatment, time $\mathrm{X}$ treatment, behaviour $\mathrm{X}$ time $\mathrm{X}$ treatment, $p<0.05$.

\subsection{Supplemental Discussion}

The expression of $\mathrm{PKM} \zeta$ protein was examined in rat hippocampal and ACC tissue using Western blot. No significant differences were noted in the protein levels of $\mathrm{PKM} \zeta$ between experimental groups from Manuscript 1 or 3 . The level of $\mathrm{PKM} \zeta$ protein in each subject was normalized to the level of GAPDH, a housekeeping protein, to account for variability in loading and transfer rates between different samples in different lanes.

Based on our c-Fos findings from the current manuscript and Manuscript 1 (Wartman and Holahan, 2013), we expected to observe increased PKM $\zeta$ levels in the hippocampus at remote compared to recent time points. With elevated c-Fos levels noted in the ACC in rats trained on two hippocampal-dependent tasks and tested recently, we hypothesized that there would be elevated levels of $\mathrm{PKM} \zeta$ in the ACC of this group compared to other recently tested groups. Our findings did not support this hypothesis. There were no significant differences between any experimental groups. Our hypotheses were similar for rats that underwent behavioural procedures from Manuscript 3. We expected rats to show increased levels of hippocampal $\mathrm{PKM} \zeta$ expression at remote compared 
to recent time points. No significant differences between groups and no effect of ACC inactivation was found on $\mathrm{PKM} \zeta$ expression.

In future studies, it may be beneficial to include a naïve control group of rats for comparison. In Manuscripts 1 and 3, all rats received at least 5 days of training on a WM task. Our Western blot technique may not have been sensitive enough to detect an increase in $\operatorname{PKM} \zeta$ expression resulting from training on subsequent tasks. Initial WM training could have resulted in $\mathrm{PKM} \zeta$ expression levels that masked any additional increases.

Although initially very promising, presently the role of PKM $\zeta$ in LTP and memory maintenance is under question. Conventional and conditional PKC $\zeta /$ PKM $\zeta$ knockout mice showed normal hippocampal synaptic transmission and performance on learning and memory tasks (Volk et al., 2013). ZIP, believed to be a selective PKM $\zeta$ pseudosubstrate inhibitor (Shema et al., 2007) resulted in LTP reversal in these knockout mice. These results suggest that ZIP has either non-specific effects or its action is not dependent on $\mathrm{PKM} \zeta$ (Volk et al., 2013). Lee et al. (2013) et al found similar findings using $\mathrm{PKC} \zeta / \mathrm{PKM} \zeta$ knockout mice. Knockout mice performed similarly to controls on tests of learning and memory. Of importance, injections of ZIP into the nucleus accumbens of knockout mice impaired reward memory. The results from Lee et al. (2013) support the findings of Volk et al. (2013), and suggest ZIP can impair memory through PKM $\zeta$ independent mechanisms. These recent findings are troubling considering the body of research involving PKM $\zeta$. Much of the research conducted and conclusions drawn about PKM $\zeta$ have resulted from inhibition of PKM $\zeta$ using ZIP (Migues et al., 2010; Pastalkova et al., 2006; Serrano et al., 2008; Shao et al., 2011; Shema et al., 2007; Yao et al., 2008). With recent results suggesting ZIP may function through $\mathrm{PKM} \zeta$ independent mechanisms, the importance of $\mathrm{PKM} \zeta$ in memory maintenance has been brought into question. However, research regarding PKMZs role in memory maintenance is not exclusively dependent on data using ZIP. As well, a different form of PKC, PKC iota, has been suggested to compensate for deficiencies in $\mathrm{PKM} \zeta$ in the above mentioned experiments. 


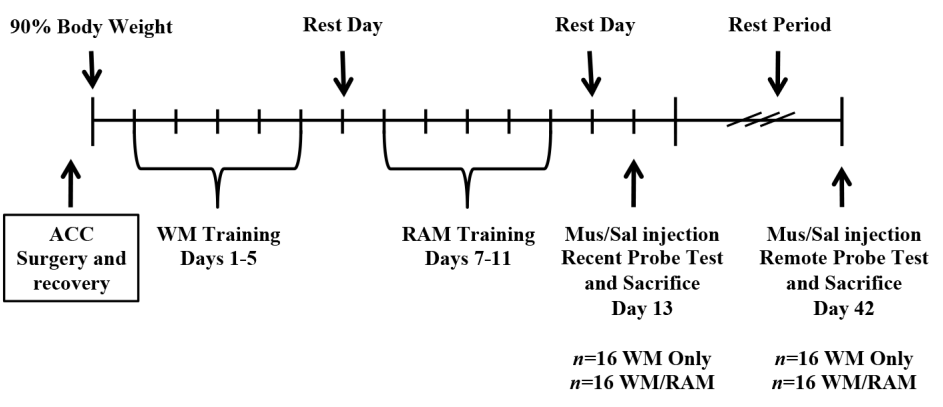

Figure 4.1: Timeline of experimental procedures. 
A
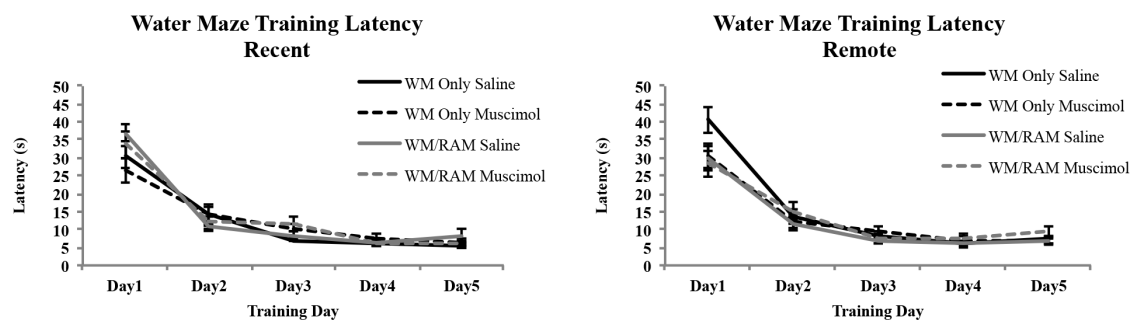

B

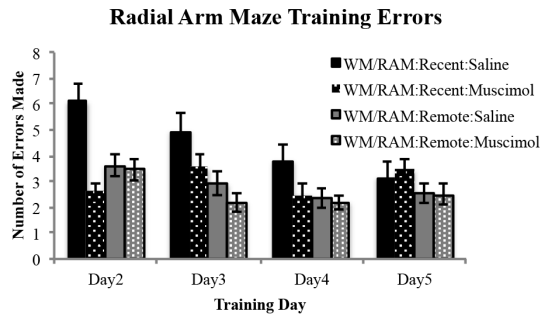

Figure 4.2: Behavioural training data. A) Average latencies to reach the hidden platform during the five days of water maze training. Recent and remote time points are plotted and examined separately to reduce visual noise. At the recent time point, a main effect of day was found. Latency to reach the hidden platform decreased across training day indicating an improvement in performance. No main effect of behaviour or treatment was found. Similarly, at the remote time point a main effect of day was found. Latency to reach the hidden platform decreased across training day indicating an improvement in performance. No main effect of behaviour or treatment was found. B) Average number of errors (unbaited arm entries and re-entries) made in the radial arm maze task during the four days of training. A main effect of day was found. Errors made decreased across training day indicating an improvement in performance. No main effect of time or treatment was found. 

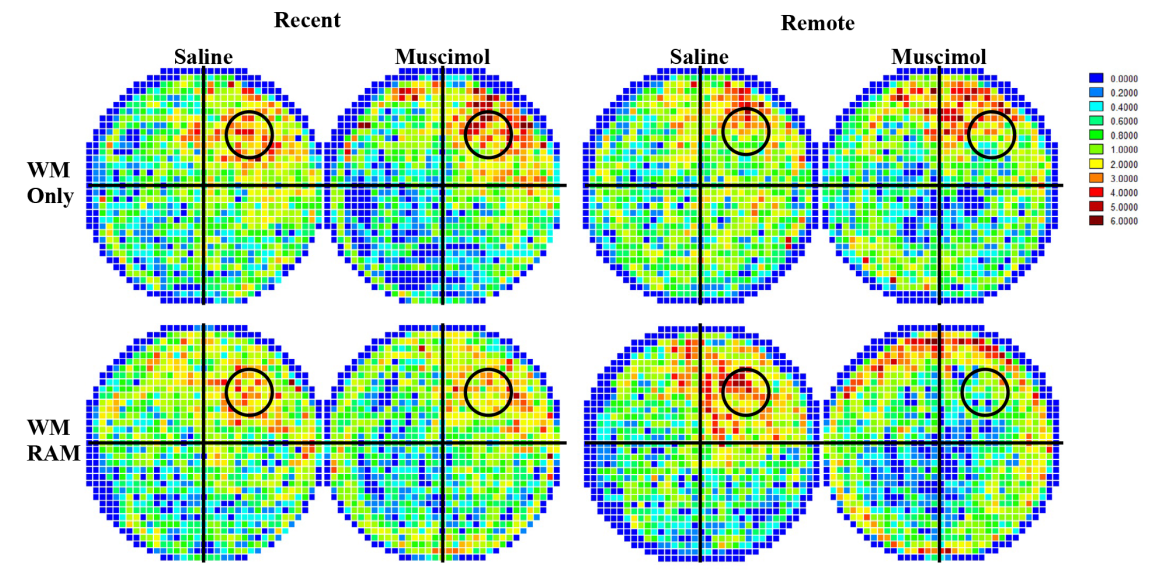

Figure 4.3: Average dwell times in discrete areas of the pool for all eight experimental groups during the 60 second water maze probe test. The scale bar colors represent average time spent in an area of the pool derived from group data. The circle represents the location of the hidden platform during training. 
A

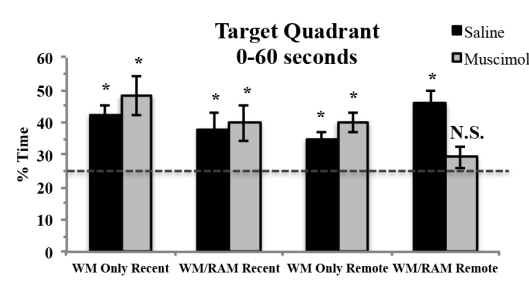

B

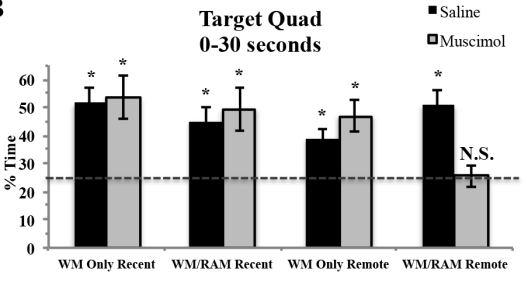

C

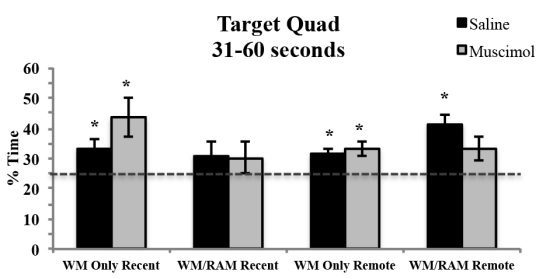

Figure 4.4: Percent of time spent swimming in the target quadrant during the 60 second probe (A), from 0-30 seconds (B) and 31-60 seconds (C). The dashed line represents chance performance (25\%). A) During the 60 second probe, all experimental groups spent above chance amounts of time swimming in the target quadrant, except rats trained on two spatial tasks, tested remotely and given a muscimol injection (WM/RAM:Remote:Muscimol). B) From 0-30 seconds, all experimental groups spent above chance amounts of time swimming in the target quadrant, except rats trained on two spatial tasks, tested remotely and given a muscimol injection (WM/RAM:Remote:Muscimol). C) From 31-60 seconds fewer groups displayed a preference for the target quadrant. * Significantly different from chance performance $(p<0.05)$. 

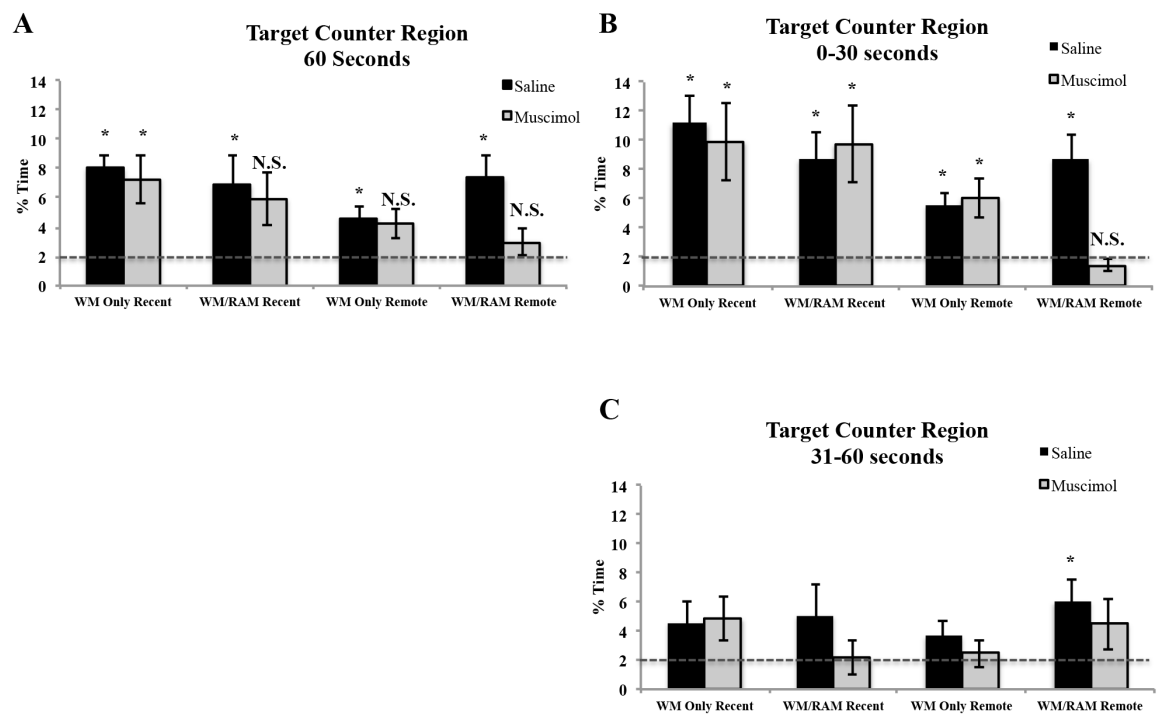

Figure 4.5: Percent of time spent swimming in the target area during the 60 second probe (A), from 0-30 seconds (B) and 31-60 seconds (C). The dashed line represents chance performance (2\%). A) During the 60 second probe, all experimental groups spent above chance amounts of time swimming in the target area, except the group trained on two spatial tasks, tested remotely and given a muscimol injection (WM/RAM:Remote:Muscimol), as well as groups WM Only:Recent:Muscimol and WM/RAM:Recent:Muscimol. B) From 0-30 seconds, all experimental groups spent above chance amounts of time swimming in the target area, except rats trained on two spatial tasks, tested remotely and given a muscimol injection (WM/RAM:Remote:Muscimol). C) From 31-60 seconds, only group WM/RAM:Remote:Saline spent above chance amounts of time in the target area. ${ }^{*}$ Significantly different from chance performance $(p<0.05)$. 
A

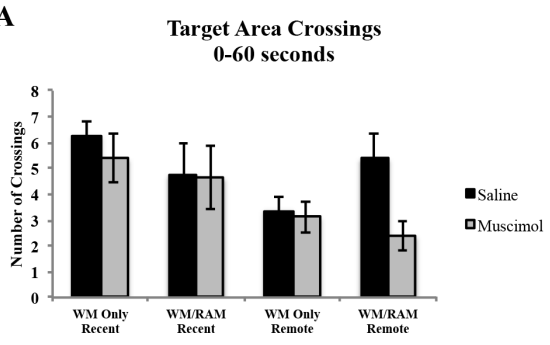

B
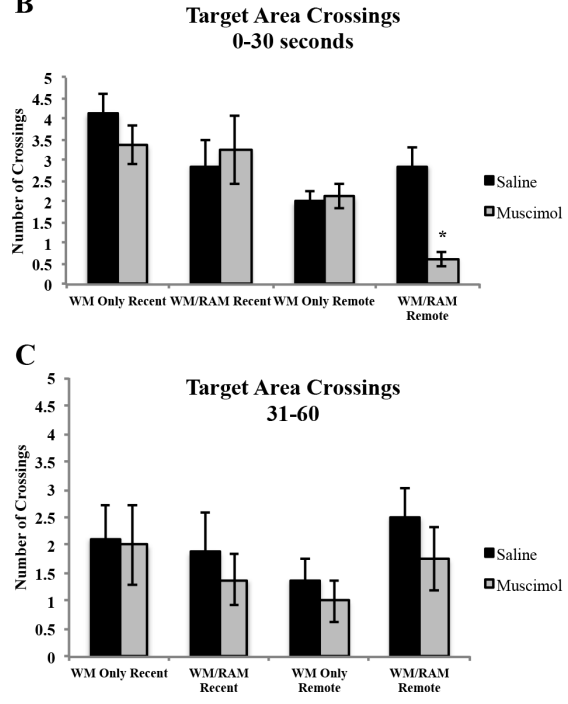

Figure 4.6: Number of crossings through the target area during the 60 second probe A), from 0-30 seconds (B) and 31-60 seconds (C) during the water maze probe test. A) During the 60 second probe, no significant differences between experimental groups were found. B) From 0-30 seconds, rats trained on two spatial tasks, tested remotely and given a muscimol injection (WM/RAM:Remote:Muscimol) had significantly fewer crossings through the target area compared to all other experimental groups. C) From 31-60 seconds, no significant differences between experimental groups were found. * Significantly different from all other experimental groups $(p=0.05)$. 


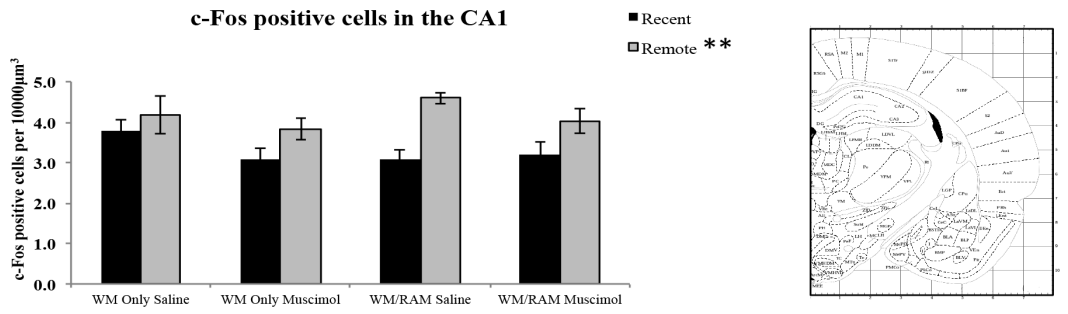

C

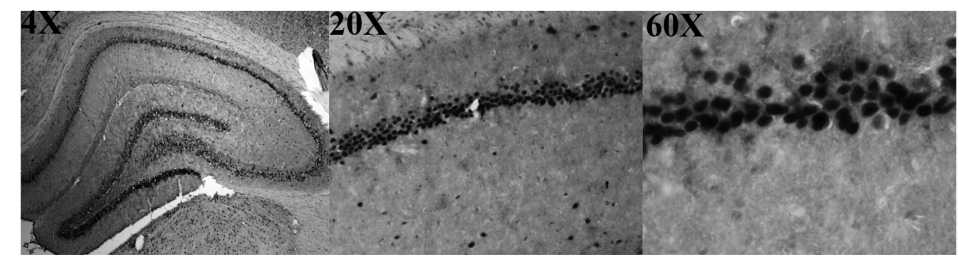

Figure 4.7: c-Fos positive cells in the CA1 of the hippocampus. A) Stereological quantification of the number of c-Fos positive cells per $10000 \mu \mathrm{m}^{3}$ in the CA1 of the hippocampus. Remotely probed rats displayed greater numbers of c-Fos positive cells compared to recently probed rats. B) Depiction of the CA1 area where c-Fos immunoreactive cells were counted (Paxinos and Watson, 2006). C) Representative images of the c-Fos in the CA1 at 4X, 20X and 60X magnification. ${ }^{* *}$ Indicates a main effect 
A

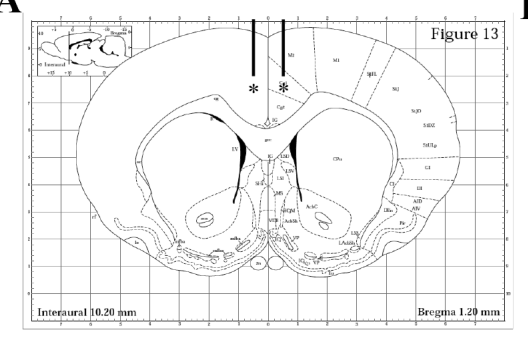

B
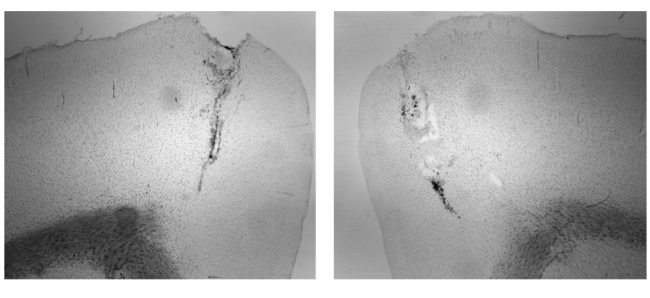

Figure 4.8: A) Depiction of the ACC where cannulas were implanted (Paxinos and Watson, 2006). B) Representative images of right and left hemisphere microinjection site (4 X magnification). 


\section{$55 \mathrm{kDa}$

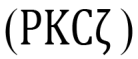 $37 \mathrm{kDa}$ (GAPDH)}

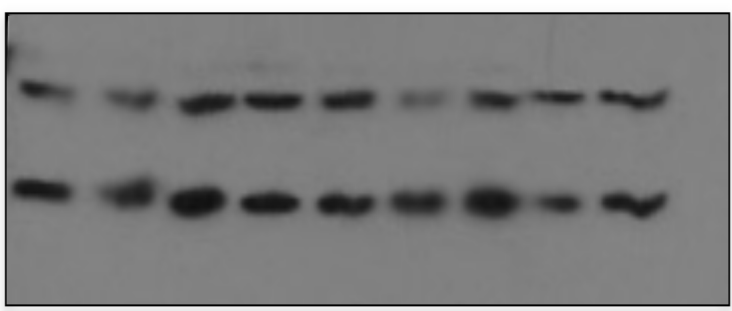

Figure 4.9: A representative developed western blot displaying bands at approximately 55 and $37 \mathrm{kDa}$, representing $\mathrm{PKC} \zeta$ and GAPDH respectively. 
A

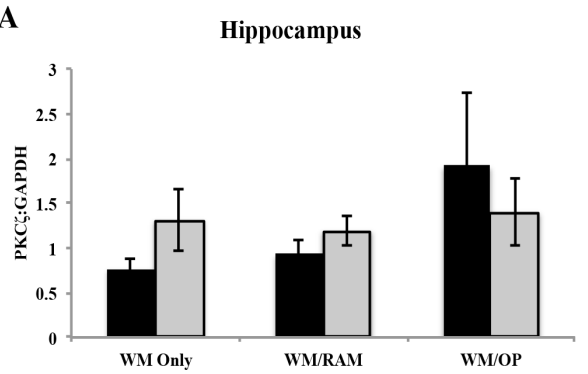

B

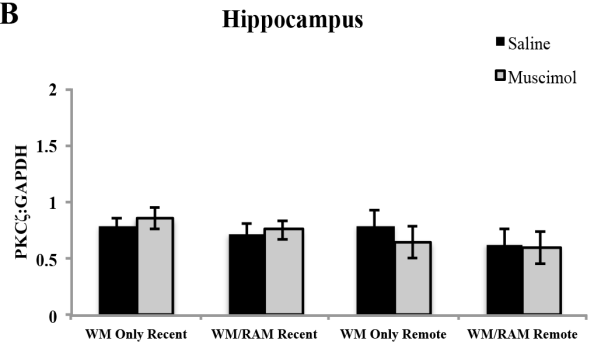

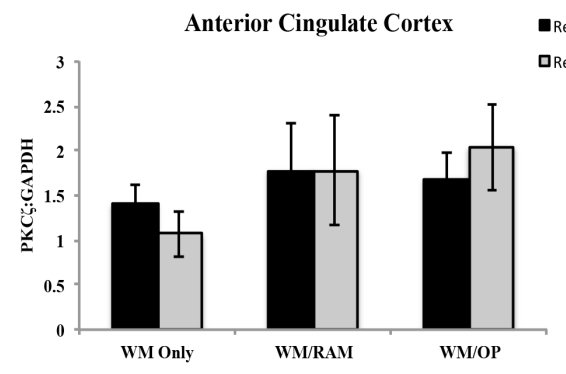

- Recent 口Remote

- 


\section{Chapter 5}

\section{General Discussion}

This thesis sought to determine whether increasing memory processing demands, in addition to the passage of time, would influence whether memory storage and retrieval were associated with ACC engagement. To this end, a novel behavioural procedure was developed to increase the processing demand placed upon the hippocampus. The present thesis demonstrates 1) continued involvement of the hippocampus in remote spatial memory processes 2) increased involvement of the ACC as time progresses and 3) increased ACC engagement at both recent and remote time points with increased memory processing demands.

\subsection{Summary of Results}

Manuscript 1 provided activational evidence for the involvement of the ACC in remote and recent memory retrieval. In agreement with past research, increased activation of the ACC was noted at remote time points (Bontempi et al., 1999; Frankland et al., 2004; Lopez et al., 2012; Maviel et al., 2004; Teixeira et al., 2006; Weible et al., 2012). The multiple memory behavioural manipulation resulted in earlier activation of the ACC when rats were trained on two spatial tasks. This earlier activation noted in the ACC was not simply due to increased behavioural training, as rats trained on one spatial and one non-spatial task did not show the same robust increase in ACC activation at the recent time point.

Manuscript 2 investigated the structural correlates associated with the multiple memory procedure. Increased dendritic complexity of ACC and hippocampal neurons were found in rats trained on two spatial tasks and euthanized at both recent and remote time points. Importantly, significant structural differences were noted consistently in the ACC at the recent time point in rats trained on two spatial tasks. This experiment did not include a probe test, and thus, morphological changes observed were likely associated with consolidation and memory storage processes. Manuscript 2 provided structural support for the functional findings from Manuscript 1 suggesting an accelerated ACC recruitment in rats trained on two spatial tasks. 
Manuscript 3 examined the necessity of the ACC in memory retrieval, through inactivation of $\mathrm{ACC}$ function prior to a WM probe test. Behavioural evidence indicated a graded effect of ACC inactivation dependent upon the number of memories processed. In agreement with past literature (Ding et al., 2008; Frankland et al., 2004; Lopez et al., 2012; Maviel et al., 2004; Teixeira et al., 2006), ACC inactivation resulted in a slight performance impairment in rats trained on one spatial task and tested remotely, while recent counterparts did not display a performance deficit. A subtle deficit in performance was noted in rats trained on two spatial tasks and tested recently. A robust deficit in performance was noted in rats trained on two spatial tasks and tested remotely. These results demonstrate a graded dependence on ACC function when rats were trained on one or two spatial tasks. The causational evidence from Manuscript 3 supports the activational results described in Manuscript 1 and the structural evidence described in Manuscript 2.

Together, these data provide evidence to suggest that the ACC becomes an important node in spatial memory consolidation, retrieval and storage processes and that the involvement of the ACC can be manipulated by increasing memory processing demands, in this case, the demand placed on the hippocampus. These data also demonstrate that the hippocampus remains involved in memory processes at remote time points.

\subsection{Consolidation Theories}

The consolidation theories presented in the introduction (The Standard Model of Consolidation, the Multiple Trace Theory, and the Cognitive Map Theory) hypothesize that the hippocampal formation integrates information initially registered in neocortical circuits to form a memory trace represented by ensembles of bound neocortical-hippocampal neurons. However, the theories differ in their hypothesis of the contribution of different brain regions to the representation of a memory as it ages. The experiments in the present thesis were not designed to specifically test any of the consolidation theories. However, we believe it is important to discuss the results with respect to the specific components of these theories in an effort to contribute and advance the pertinent literature.

The SMC theory posits that hippocampal contribution gradually diminishes while cortical contribution increases as consolidation processes proceed, indicating a time-dependent role of the hippocampus in memory storage. In line with the SMC, our results suggest increased involvement of the ACC as time progresses. Our results show that increased processing demand on the hippocampus led to accelerated recruitment of the ACC at the recent time point. These findings support a progressive strengthening of cortical connections as proposed by the SMC. However, increased levels of activity were observed in the hippocampus at remote, compared to recent, time points and structural findings showed increased dendritic complexity at both time points in the hippocampus. These results suggest there is a continued and sustained involvement of the hippocampus in memory storage and retrieval at recent and remote time points. This 
is inconsistent with the time-limited role of the hippocampus proposed by the SMC. In line with other research, memory retention was assessed only up to a time point of 37 days following the end of training. However, it is possible hippocampal involvement could completely diminish at later time points.

The MTT suggests vivid and detailed memories, such as autobiographical (episodic) memories, always require hippocampal function. The MTT hypothesizes that older memories are represented by a greater number of traces and are stronger than recent memories that are represented by fewer traces. Our finding of increased hippocampal activation at remote compared to recent time points provides support for the MTT hypothesis. The MTT suggests that spatial memories, like other memories, can be represented in extra-hippocampal regions, but when stored outside of the hippocampus these memories become generic, schematic and lacking in detail and salience, while hippocampal representation would be required for contextual cues rich in detail and integration (Moscovitch et al., 2006, 2005; Winocur et al., 2010). Inactivation of the ACC produced graded behavioural impairments, which suggested a progression of cortical consolidation processes. This is not hypothesized by the MTT for vivid episodic memories. However, the vividness of the memory expressed was not examined in any way in this thesis. The direct contribution of the hippocampus was not examined either, as no hippocampal lesions or inactivations were included making it difficult to fully conclude whether the present set of experiments support all aspects of the MTT.

The CMT does not differentiate between recent and remote memories. Instead, it suggests the hippocampus is required for allocentric spatial processing that provides the context for episodic events, and thus the hippocampus is important for recent and remote retrieval of spatial memories. Although the direct contribution of the hippocampus was not examined in this thesis, activational and structural analysis suggested involvement of the hippocampus at recent and remote time points. The direct contribution of the ACC was examined and findings from all studies suggested the ACC was involved in the retrieval and storage of a spatial memory in a time dependent and hippocampal-demand dependent fashion. The CMT does not account for all of the findings presented in the present thesis.

\subsection{Complementary Memory Systems}

It is well established that the hippocampus is required to encode new memories. This thesis examined the consolidation of memories, hypothesized to become increasingly more reliant on the ACC as time and hippocampal-demand increased. This begs two important questions, initially posed by McClelland et al. (1995): If memory becomes dependent on cortical areas, why is a hippocampal system required in the first place-why are memories transferred from one area to another? Why does dependence on cortical areas take such a long time? Computational models have provided a rationale to help answer these questions (McClelland et al., 1995; Roxin and Fusi, 2013; Takashima et al., 2009). As 
the hippocampus is required to form new declarative memories it makes sense that the hippocampus be a highly plastic structure able to encode information rapidly. However, rapid plasticity is not conducive to long-term storage, as the acquisition of new information may disturb the retention of old information. If the cortex represents a permanent repository for information, it makes sense for this area to be less plastic and slower to encode new information. This would allow the integration of new information into existing circuits, without interference. This rationale provides the basis for why memories may not be stored in the hippocampus for the long-term.

The present thesis provides evidence that shows the ACC is recruited at earlier time points when hippocampal-processing demand is taxed. If the ACC is largely time-dependent in its involvement in memory processes, why can recruitment of this region be manipulated by hippocampal-processing demand? Increased processing demands on the hippocampus, particularly by tasks that require explicit hippocampal functioning for their performance, may act to consolidate memories to cortical areas more quickly in order to free processing space for incoming information. This does not necessarily mean that consolidated memories become completely independent from the hippocampus. In fact, we provide evidence that suggests spatial memory retrieval engages hippocampal activation more strongly at remote compared to recent time points. However, memories for spatial tasks may represent a special case, such that these memories remain associated with the hippocampus for an extended period of time.

\subsection{The Special Case of Spatial Memory}

One of the objectives of this thesis was to shed more light on remote spatial memory processes. As Winocur et al. (2010) pointed out, there has been much interest in spatial memories, but little research into the representation of remote spatial memories. As spatial memories are intrinsically linked to hippocampal function, it is of interest to determine their representation at remote time points. As described previously, conflict exists in human and animal research concerning whether remote spatial memories can be represented independently of the hippocampus. In the present thesis, we focused on the role of the cortex (specifically the ACC) in the representation of remote spatial memories. This thesis showed deficits in spatial memory retrieval when the ACC was pharmacologically inactivated. Hippocampal function was not interfered with and levels of hippocampal activation were similar to those seen in control animals and past experiments. This suggests that some critical aspects of memory become represented within cortical ACC circuits, with hippocampal function insufficient to produce an intact memory representation. The graded nature of the performance deficit seen suggests time and hippocampal processing demand impact the rate of dependence on the ACC. 


\subsection{Relevant Findings and Future Directions}

Factors that lead to an accelerated rate of consolidation to extra-hippocampal areas have not been researched extensively. However, Tse et al. (2007) carried out an elegant series of experiments that demonstrated accelerated consolidation to extra-hippocampal regions when an associative schema existed into which new information could be incorporated. They found hippocampal-independent memory existed at 48 hours following learning of a hippocampal-dependent paired associations task when previous paired associations in the same environment had been learned. They concluded that the rate at which systems consolidation occurs in the cortex could be influenced by what is already known. An additional study by this team showed the ACC was part of a neocortical network for schema storage (Wang et al., 2012). The present thesis showed that learning of a spatial task, followed by learning of a different spatial task with similar demands, accelerated the rate of ACC recruitment in memory processes. Based on the finding from Tse et al. (2007) it would be interesting to examine the activation and necessity of the ACC in the subsequent task learned in the multiple memory behavioural procedure.

Recent research has reached similar conclusions as those presented in the present thesis. Bonaccorsi et al. (2013) examined the effect of environmental enrichment on systems consolidation. Mice were trained on a water maze task and tested 1, 10, 20, 30 or 50 days following the last day of training. c-Fos activation was examined in the hippocampus and cortical regions. Activity in the dorsal CA1 region of the hippocampus did not differ between standard housed and environmentally enriched mice. Activation in the CA1 increased with increasing retention intervals up to 30 days. A remarkably similar pattern of c-Fos activity was found in the present thesis, with an increase in CA1 activation noted at remote time points compared to recent time points, but no significant differences between different behavioural manipulations (e.g. one or two training tasks). Similar to past research indicating a time-dependent recruitment of the ACC in remote memory processing (Bontempi et al., 1999; Frankland et al., 2004; Lopez et al., 2012; Maviel et al., 2004; Teixeira et al., 2006; Weible et al., 2012), Bonaccorsi et al. (2013) noted increased ACC activity in standard housed mice at 30 days following WM learning. Importantly, Bonaccorsi et al. (2013) also showed that medial prefrontal cortex activation, including the ACC, was noticeable at shorter time intervals (10 days following WM training) in environmentally enriched rats compared to standard housed rats, evidence of accelerated $\mathrm{mPFC}$ recruitment. The present thesis noted ACC activation at 8 days following WM training in rats trained on two spatial tasks. Thus, early ACC recruitment is noted following environmental enrichment and training on two spatial tasks.

Environmental enrichment as outlined by Bonaccorsi et al (2013) is a combination of social and inanimate stimulation that may serve to enhance cognitive activity, exploration and physical activity. Environmental enrichment promotes

the survival of newly generated neurons and is thus implicated in neurogenesis (van Praag et al., 2000). In the present thesis, behavioural manipulation of the 
number and type of tasks used resulted in similar findings as those reported by Bonaccorsi et al. (2013). There are similarities between exposure to environmental enrichment and training on the multiple memory procedure. In the present thesis, training on multiple tasks occurred in different rooms, providing environmental stimulation and exploration of novel environments. Training on different tasks likely increased cognitive activity, as each task required the learning of specific task demands. The WM and RAM tasks were both spatial tasks that included a physical component, while the OP task was non-spatial and did not place specific physical activity demands on the rat. The components of environmental enrichment are similar to the components outlined in the multiple memory behavioural procedure, particularly in the group trained on the WM and RAM tasks.

Bonaccorsi et al. (2013) suggested hippocampal neurogenesis, resulting from environmental enrichment, could underlie accelerated ACC recruitment. Kitamura et al. (2009) provide compelling support for this hypothesis. This team examined the effect of neurogenesis on hippocampal-dependency of a memory. Decreased neurogenesis was associated with a prolonged period of hippocampaldependency of an associative fear memory. Increased neurogenesis was associated with an accelerated decay rate of hippocampal-dependency without a corresponding loss in memory, indicating the memory had come to depend on extra-hippocampal components. These findings suggest that hippocampal neurogenesis plays a role in determining the period of hippocampal-dependency of a memory.

All three behavioural tasks used in the multiple memory behavioural procedure have been associated with neurogenesis (WM:(Ambrogini et al., 2000; Gould et al., 1999; Kee et al., 2007; Kempermann and Gage, 2002) RAM: (Ambrogini et al., 2000; Epp et al., 2010; Gould et al., 1999; Hairston et al., 2005; Leuner et al., 2004) OP: (Rapanelli et al., 2011). Future studies should examine neurogenesis rates in the different manipulations of the multiple memory behavioural procedure and determine whether accelerated ACC recruitment occurs in a neurogenesis-dependent fashion. An important question to explore is, does neurogenesis contribute to accelerate the dependence of memory on cortical structures? And if so, how? A recent review by Kitamura and Inokuchi (2014) suggested that hippocampal neurogenesis might help to generate sharp-wave ripples (SPWs) in the CA3, which could drive CA1 reactivation leading to intercortical plasticity. This could promote cortical consolidation during periods of inactivity, leading to accelerated cortical recruitment. Kitamura and Inokuchi (2014) also suggest that hippocampal neurogenesis is important in erasing old memories in the hippocampus. The present thesis does not provide explicit evidence to support this theory of hippocampal disengagement, as functional and structural findings suggest continued involvement of the hippocampus in memory processes. However, the hypothesis that cortical recruitment is driven by neurogenesis is a compelling argument, and future investigations should include experiments to test this hypothesis in regards to the multiple memory behavioural procedure.

Past research has suggested there is a progressive reduction in hippocampal 
activation as a memory ages (Bontempi et al., 1999; Frankland and Bontempi, 2005; Maviel et al., 2004). The present thesis, along with other recent studies (Bonaccorsi et al., 2013; Lopez et al., 2012; Schlesiger et al., 2013; Wartman and Holahan, 2013), does not provide support for a reduction in hippocampal activation. In fact, results from this thesis showed greater hippocampal activation at remote, compared to recent, time points. It is likely that discrepancies concerning hippocampal activation result from differences in task complexity, cue saliency, environmental influences and general procedures. For example, Lopez et al. (2012) examined performance on a water maze task in two different contexts: a context with salient distal cues and a context with less salient and fewer distal cues. Their results showed that the requirement of brain regions such as the $\mathrm{mPFC}$ and hippocampus during remote spatial memory retrieval depend on environmental conditions such as cue saliency and the complexity of the task. These findings highlight the importance of environmental conditions under which the memory is formed and recalled. Remote hippocampal activation may result from a greater burden on hippocampal function resulting from a complex spatial environment and navigational demands on the WM task. The burden on hippocampal function may be less with more recent memories. However, Schlesiger et al. (2013) found increased hippocampal activation at a remote time point in a modified RAM task where there was very low demand for spatial navigation, indicating remote hippocampal activation does not result from high navigational demands alone. The modified RAM included the use of multiple start locations and single trial retention testing, creating a RAM task with demands more similar to a WM task, but without additional navigational burdens. There was greater CA1 activation at remote compared to recent recall time points. Schlesiger et al. (2013) suggested the increased hippocampal activation at remote time points might reflect greater pattern completion or relearning requirements following longer time intervals. It should be considered that spatial memory represents a special case, whereby the hippocampus, as a result of its role in spatial processes, remains involved in the memory trace. Additionally, it should be stated that the involvement and activation of the hippocampus at a remote time point does not mean it is critical or necessary for remote memory processes, as the requirement for hippocampal function was not explicitly examined in the present thesis.

A point of interest is determining whether the ACC is involved in storing the memory trace or retrieving and integrating memories from distributed cortical regions. The structural evidence in this thesis was gathered from rats that received training on the multiple memory procedure but did not receive a probe test prior to sacrifice. Training on two spatial tasks was sufficient to induce significant structural changes at recent and remote time points of consolidation. Pronounced and sustained significant morphological increases in dendritic complexity in ACC neurons at the recent and remote time point in rats trained on two spatial tasks likely indicate ACC involvement in storage processes. Similar significant increases in dendritic complexity were found in the hippocampus in rats trained on two spatial tasks. These structural changes occurred in the absence of retrieval processes, suggesting a long-lasting involvement of the ACC 
and hippocampus in memory processes.

The reliability of the findings described in this thesis should be tested utilizing other behavioural manipulations. For example, reversing the order of the training tasks would be valuable such that rats are trained on the RAM followed by the WM, with a RAM probe test. It would also be beneficial to use a modified RAM task with task demands similar to the WM, as outlined by Schlesiger et al. (2013). The multiple memory procedure used in this thesis allows for consolidation of task learning across five days of the training procedure. It would be interesting to examine the effect of accelerating the training procedures on consolidation processes. In this sense, it would be valuable to include tasks that require only a single day for training. This would allow behavioural, synaptic and systems level early consolidation processes, resulting from the rapid consolidation of memories, to be examined.

The graded behavioural deficits noted from ACC inactivation in Manuscript 3 should be expanded to investigate the effect of ACC inactivation on rats trained on one spatial and one non-spatial task (e.g. WM/OP). It would also be of interest to probe the memory of the second task learned, and examine the functional and behavioural outcomes resulting from ACC inactivation. The structural analysis conducted in the present thesis provided interesting and valuable findings. An interesting continuation of this work would include classifying and quantifying spine types e.g. mushroom spines, thin spines and stubby spines. Quantity of spine type may provide valuable insight into the function of that area.

\subsection{Conclusion}

The present thesis provides evidence that increasing the processing demands placed on the hippocampus can effect time-dependent spatial memory system consolidation. Training on two spatial tasks prompted earlier recruitment of the ACC, which suggests accelerated dependence of spatial memory on this cortical area. We do not provide evidence to suggest this early recruitment of the ACC implies an earlier independence from the hippocampus, as results show the hippocampus is involved in spatial memory processes regardless of the age of the memory. Structural findings indicate the ACC likely represents an area for permanent memory storage. A critical role of the ACC has been demonstrated in remote spatial memory processes, with its recruitment being both time-dependent and hippocampal-demand dependent.

In addition to shedding light on the mechanisms involved in remote memory storage, this thesis demonstrates the processes involved in the consolidation of memories that may be more similar to the natural human condition. Laboratory animals are typically behaviourally naïve, trained and tested on a single task, without the opportunity to experience multiple environments and events. This proves useful in laboratory procedures, but differs from the real world. By exposing rats to more than one task, and in effect challenging the processing systems in their brains, we may uncover a more realistic time-line of consolidation 
processes, improving and advancing our knowledge of such processes. 


\section{Chapter 6}

\section{Appendix A- Copyright Documentation}

\subsection{Permission from Neurobiology of Learning and Memory}

The following has been extracted from the Elsevier website (http://www.elsevier. com/journal-authors/author-rights-and-responsibilities? $\mathrm{a}=105167$ \#published-journalarticle):

A published journal article (PJA) is the definitive final record of published research that appears or will appear in the journal and embodies all value-adding publisher activities including copy-editing, formatting and (if relevant) pagination. An author may use the PJA for personal use and internal institutional use.

Use by an author in the authors classroom teaching (including distribution of copies, paper or electronic), distribution of copies to research colleagues for their personal use, use in a subsequent compilation of the authors works, inclusion in a thesis or dissertation, preparation of other derivative works such as extending the article to book-length form, or otherwise using or re-using portions or excerpts in other works (with full acknowledgment of the original publication of the article). 


\subsection{Permission from Frontiers in Behavioural Neu- roscience}

The following has been extracted from the Frontiers website (http://www.frontiersin. org/about/faq):

Under the Frontiers Terms and Conditions, authors retain the copyright to their work. All Frontiers articles are Open Access and distributed under the terms of the Creative Commons Attribution License, (CC-BY 3,0), which permits the use, distribution and reproduction of material from published articles, provided the original authors and source are credited, and subject to any copyright notices concerning any third-party content. 


\subsection{Co-author Permission Statements}

To Whom It May Concern:

I, Matthew Holahan, hereby grant Brianne Wartman permission to use all contents associated with the manuscript, The use of sequential hippocampal-dependent and-nondependent tasks to study the activation profile of the anterior cingulate cortex during recent and remote memory test, published in Neurobiology of Learning and Memory, as part of her $\mathrm{PhD}$ dissertation.

Signature:

Date: Apr: 28,2014 
To Whom It May Concern:

I, Matthew Holahan, hereby grant Brianne Wartman permission to use all contents associated with the manuscript, The impact of multiple memory formation on dendritic complexity in the hippocampus and anterior cingulate cortex assessed at recent and remote time points, published in Frontiers in Behavioural Neuroscience, as part of her $\mathrm{PhD}$ dissertation.

signature: $\mathrm{Feg}$

Date: April 28, 2014 
To Whom It May Concern:

I, Matthew Holahan, hereby grant Brianne Wartman permission to use all contents associated with the manuscript, Inactivation of the anterior cingulate reveals enhanced reliance on cortical networks for remote spatial memory retrieval after multiple memory processing, submitted for publication in PLOS ONE, as part of her PhD dissertation.

signature: Hutgh

Date: Apc.l 28,2014 
To Whom It May Concern:

I, Jennifer Gabel, hereby grant Brianne Wartman permission to use all contents associated with the manuscript, Inactivation of the anterior cingulate reveals enhanced reliance on cortical networks for remote spatial memory retrieval after multiple memory processing, submitted for publication in PLOS ONE, as part of her $\mathrm{PhD}$ dissertation.

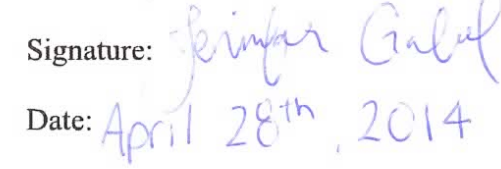


Chapter 7

\section{Appendix B-Supervisor Statement}


Carleton

Canada's Capital University

April 14, 2014

I certify that this thesis conforms in all respects to the regulations of Carleton University and that I have verified the following requirements:

1. The thesis comprises original work which the student was fully involved in setting up and conducting. Brianne not only set up and conducted all work reported in a largely independent manner (with guidance provided by me and minor assistance provided by students), she also collected and analyzed all data.

2. The student provided the conception and design of the experiments, provided analysis and interpretation of data, drafted and revised the manuscripts and submitted them serving as corresponding author and dealing with reviewer comments. The thesis supervisor edited written text, provided final approval for submitted and revised manuscripts and provided overall guidance in data analysis and interpretation.

3. The information provided by the student in the preface is accurate.

4. The thesis reflects work done during the period of candidature and no work presented has contributed to an award of a previous qualification.

5. Research data collected, used and maintained in the conduct of this thesis have been deposited with the supervisor having administrative oversight of the candidature in accordance with legal and ethical requirements. Research data and records will be retained and accessible for a minimum of 5 years from the date the thesis is passed or from the date of publication.

Sincerely,

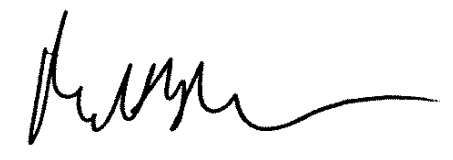

Matthew R. Holahan, PhD

Dept of Neuroscience/ Carleton University

325 Life Sciences Research Building

1125 Colonel By Drive

Ottawa, ON Canada K1S 5B6

tele: 613-520-2600 ext 1543

matthew_holahan@carleton.ca 


\section{Bibliography}

Abraham, W. C. and Williams, J. M. (2003). Properties and mechanisms of LTP maintenance. The Neuroscientist : a review journal bringing neurobiology, neurology and psychiatry, 9(6):463-74.

Aggleton, J. P. and Brown, M. W. (1999). Episodic memory, amnesia, and the hippocampal-anterior thalamic axis. The Behavioral and brain sciences, 22(3):425-44; discussion 444-89.

Aharoni, E., Vincent, G. M., Harenski, C. L., Calhoun, V. D., SinnottArmstrong, W., Gazzaniga, M. S., and Kiehl, K. A. (2013). Neuroprediction of future rearrest. Proceedings of the National Academy of Sciences of the United States of America, 110:6223-6228.

Alberini, C. M., Milekic, M. H., and Tronel, S. (2006). Mechanisms of memory stabilization and de-stabilization. Cellular and molecular life sciences, 63(9):999-1008.

Alexander, K. A., Wakim, B. T., Doyle, G. S., Walsh, K. A., and Storm, D. R. (1988). Identification and characterization of the calmodulin-binding domain of neuromodulin, a neurospecific calmodulin-binding protein. The Journal of biological chemistry, 263(16):7544-9.

Allman, J. M., Hakeem, A., Erwin, J. M., Nimchinsky, E., and Hof, P. (2001). The anterior cingulate cortex. The evolution of an interface between emotion and cognition. Annals of the New York Academy of Sciences, 935:107-117.

Amaral, D. G., Dolorfo, C., and Alvarez-Royo, P. (1991). Organization of CA1 projections to the subiculum: a PHA-L analysis in the rat. Hippocampus, $1: 415-436$

Ambrogini, P., Cuppini, R., Cuppini, C., Ciaroni, S., Cecchini, T., Ferri, P., Sartini, S., and Del Grande, P. (2000). Spatial learning affects immature granule cell survival in adult rat dentate gyrus. Neuroscience letters, 286(1):21-4.

Anagnostaras, S. G., Maren, S., and Fanselow, M. S. (1999). Temporally graded retrograde amnesia of contextual fear after hippocampal damage in rats: within-subjects examination. The Journal of neuroscience, 19(3):1106-14. 
Andersen, P., Sundberg, S. H., Sveen, O., Swann, J. W., and Wigström, H. (1980). Possible mechanisms for long-lasting potentiation of synaptic transmission in hippocampal slices from guinea-pigs. The Journal of physiology, 302:463-82.

Anderson, K., Bones, B., Robinson, B., Hass, C., Lee, H., Ford, K., Roberts, T.-A., and Jacobs, B. (2009). The morphology of supragranular pyramidal neurons in the human insular cortex: a quantitative Golgi study. Cerebral cortex, 19(9):2131-44.

Annese, J., Schenker-Ahmed, N. M., Bartsch, H., Maechler, P., Sheh, C., Thomas, N., Kayano, J., Ghatan, A., Bresler, N., Frosch, M. P., Klaming, R., and Corkin, S. (2014). Postmortem examination of patient H.M.'s brain based on histological sectioning and digital 3D reconstruction. Nature communications, 5:3122.

Ascher, B. Y. P. and Nowak, L. (1988). The role of divalent cations in the Nmethyl-D-aspartate responses of mouse central neurones in culture. Journal of physiology, Paris, 399:247-266.

Ashby, M. C., Maier, S. R., Nishimune, A., and Henley, J. M. (2006). Lateral diffusion drives constitutive exchange of AMPA receptors at dendritic spines and is regulated by spine morphology. The Journal of neuroscience, 26(26):7046-55.

Atkinson, R. and Shiffrin, R. (1971). The control processes of short-term memory. Technical report.

Atkinson, R. C. and Shiffrin, R. M. (1968). Some speculations on storage and retrieval processes in long-term memory. In Research on human decision making, number 127, pages 1-24.

Azevedo, F. a. C., Carvalho, L. R. B., Grinberg, L. T., Farfel, J. M., Ferretti, R. E. L., Leite, R. E. P., Jacob Filho, W., Lent, R., and Herculano-Houzel, S. (2009). Equal numbers of neuronal and nonneuronal cells make the human brain an isometrically scaled-up primate brain. The Journal of comparative neurology, 513(5):532-41.

Bailey, C. H. and Eric, R. (1993). Structural Changes Accompanying Memory Storage. Annual review of physiology, 55:397-426.

Barbizet, J. (1963). Defect of memorizing of hippocampal-mammillary origin: a review. Journal of neurology, neurosurgery, and psychiatry, 26:127-35.

Barr, W. B., Goldberg, E., Wasserstein, J., and Novelly, R. A. (1990). Retrograde amnesia following unilateral temporal lobectomy. Neuropsychologia, 28(3):243-55. 
Bartsch, T., Schönfeld, R., Müller, F. J., Alfke, K., Leplow, B., Aldenhoff, J., Deuschl, G., and Koch, J. M. (2010). Focal lesions of human hippocampal CA1 neurons in transient global amnesia impair place memory. Science, 328(5984):1412-5.

Bashir, Z. I., Tam, B., and Collingridge, G. L. (1990). Activation of the glycine site in the NMDA receptor is necessary for the induction of LTP. Neuroscience letters, 108(3):261-6.

Basi, G. S., Jacobson, R. D., Virág, I., Schilling, J., and Skene, J. H. (1987). Primary structure and transcriptional regulation of GAP-43, a protein associated with nerve growth. Cell, 49(6):785-91.

Basil, J. A., Kamil, A. C., Balda, R. P., and Fite, K. V. (1996). Differences in hippocampal volume among food storing corvids. Brain, Behavior and Evolution, 47:156-164.

Bayley, P. J., Gold, J. J., Hopkins, R. O., and Squire, L. R. (2005). The neuroanatomy of remote memory. Neuron, 46(5):799-810.

Bayley, P. J., Hopkins, R. O., and Squire, L. R. (2003). Successful recollection of remote autobiographical memories by amnesic patients with medial temporal lobe lesions. Neuron, 38(1):135-44.

Bayley, P. J., Hopkins, R. O., and Squire, L. R. (2006). The fate of old memories after medial temporal lobe damage. The Journal of neuroscience, 26(51):13311-7.

Bear, M. F. and Abraham, W. C. (1996). Long-term depression in hippocampus. Annual review of neuroscience, 19:437-62.

Bednarek, E. and Caroni, P. (2011). $\beta$-Adducin is required for stable assembly of new synapses and improved memory upon environmental enrichment. Neuron, 69(6):1132-46.

Benfenati, F. (2007). Synaptic plasticity and the neurobiology of learning and memory. Acta bio-medica : Atenei Parmensis, 78 Suppl 1:58-66.

Benhamou, S. and Poucet, B. (1995). A comparative analysis of spatial memory processes. Behavioural Processes, 35(1-3):113-126.

Benowitz, Larry, I. and Routtenberg, A. (1997). GAP-43: an intrinsic determinant of neuronal development and plasticity. Trends in neurosciences, 20:84-91.

Bindman, L. J., Lippold, O. C., and Milne, A. R. (1979). Prolonged changes in excitability of pyramidal tract neurones in the cat: a post-synaptic mechanism. The Journal of physiology, 286:457-77. 
Bingman, V. P., Ioalé, P., Casini, G., and Bagnoli, P. (1990). The avian hippocampus: evidence for a role in the development of the homing pigeon navigational map. Behavioral neuroscience, 104(6):906-11.

Blackstad, T. W., Brink, K., Hem, J., and Jeune, B. (1970). Distribution of hippocampal mossy fibers in the rat: an experimental study with silver impregnation methods. Journal of comparative neurology, 138:433-450.

Bliss, T. V. and Gardner-Medwin, A. R. (1973). Long-lasting potentiation of synaptic transmission in the dentate area of the unanaestetized rabbit following stimulation of the perforant path. The Journal of physiology, 232(2):35774 .

Bliss, T. V., Lancaster, B., and Wheal, H. V. (1983). Long-term potentiation in commissural and Schaffer projections to hippocampal CA1 cells: an in vivo study in the rat. The Journal of physiology, 341:617-26.

Bliss, T. V. P. and Collingridge, G. L. (1993). A synaptic model of memory: long-term potentiation in the hippocampus. Nature, 361:31-39.

Bliss, T. V. P. and Lø mo, T. (1973). Long-lasting potentiation of synaptic transmission in the dentate area of the anaesthetized rabbit following stimulation of the perforant path. Journal of Physiology, 232:331-356.

Blum, I. D., Lamont, E. W., Rodrigues, T., and Abizaid, A. (2012). Isolating neural correlates of the pacemaker for food anticipation. PloS one, 7(4):e36117.

Bock, J., Gruss, M., Becker, S., and Braun, K. (2005). Experience-induced changes of dendritic spine densities in the prefrontal and sensory cortex: correlation with developmental time windows. Cerebral cortex, 15(6):802-8.

Bohbot, V. D., Iaria, G., and Petrides, M. (2004). Hippocampal function and spatial memory: evidence from functional neuroimaging in healthy participants and performance of patients with medial temporal lobe resections. Neuropsychology, 18(3):418-25.

Bolhuis, J. J. and Stewart, C. A. (1994). Retrograde amnesia and memory reactivation in rats with ibotenate lesions to the hippocampus or subiculum. The Quarterly journal of Experimental Psychology, 47B(October 2012):129150 .

Bonaccorsi, J., Cintoli, S., Mastrogiacomo, R., Baldanzi, S., Braschi, C., Pizzorusso, T., Cenni, M. C., and Berardi, N. (2013). System Consolidation of Spatial Memories in Mice: Effects of Enriched Environment. Neural plasticity, 2013:956312.

Bontempi, B., Laurent-Demir, C., Destrade, C., and Jaffard, R. (1999). Timedependent reorganization of brain circuitry underlying long-term memory storage. Nature, 400(6745):671-5. 
Bouffard, J. P. and Jarrard, L. E. (1988). Acquisition of a complex place task in rats with selective ibotenate lesions of hippocampal formation: combined lesions of subiculum and entorhinal cortex versus hippocampus. Behavioral neuroscience, 102(6):828-34.

Bourne, J. and Harris, K. M. (2007). Do thin spines learn to be mushroom spines that remember? Current opinion in neurobiology, 17(3):381-6.

Bourne, J. N. and Harris, K. M. (2008). Balancing structure and function at hippocampal dendritic spines. Annual review of neuroscience, 31:47-67.

Bramham, C. R., Alme, M. N., Bittins, M., Kuipers, S. D., Nair, R. R., Pai, B., Panja, D., Schubert, M., Soule, J., Tiron, A., and Wibrand, K. (2010). The Arc of synaptic memory. Experimental brain research, 200(2):125-40.

Bramham, C. R., Worley, P. F., Moore, M. J., and Guzowski, J. F. (2008). The immediate early gene Arc/Arg3.1. Journal of Neuroscience, 28(46):1176011767 .

Brandeis, R., Brandys, Y., and Yehuda, S. (1989). The use of the Morris Water Maze in the study of memory and learning. The International journal of neuroscience, 48(1-2):29-69.

Bright, P., Buckman, J., Fradera, A., Yoshimasu, H., Colchester, A. C. F., and Kopelman, M. (2006). Retrograde amnesia in patients with hippocampal, medial temporal, temporal lobe, or frontal pathology. Learning \& $\mathcal{E}$ Memory, 13:545-557.

Broadbent, N. J., Squire, L. R., and Clark, R. E. (2004). Spatial memory, recognition memory, and the hippocampus. Proceedings of the National Academy of Sciences of the United States of America, 101(40):14515-20.

Broadbent, N. J., Squire, L. R., and Clark, R. E. (2006). Reversible hippocampal lesions disrupt water maze performance during both recent and remote memory tests. Learning $\& 3$ Memory, 13:187-191.

Brodmann, K. (1909). Vergleichende lokalisationslehre der grosshirn- rinde in ihren prinzipien dargestellt auf grund des zellsenbaues. Barth, Leipzig.

Bruce, D. (2001). Fifty Years Since Lashley's In Search of the Engram: Refutations and Conjectures Fifty Years Since Lashley's In Search of the Engram: Refutations and Conjectures. Journal of the History of the Neurosciences : Basic and Clinical Perspectives, 10(3):308-318.

Buell, S. J. (1982). Golgi-Cox and rapid golgi methods as applied to autopsied human brain tissue: widely disparate results. Journal of neuropathology and experimental neurology, 41(5):500-7.

Burgess, N. (2008). Spatial cognition and the brain. Annals of the New York Academy of Sciences, 1124:77-97. 
Burgess, N., Maguire, E. a., and O'Keefe, J. (2002). The human hippocampus and spatial and episodic memory. Neuron, 35(4):625-41.

Burwell, R. D., Bucci, D. J., Sanborn, M. R., and Jutras, M. J. (2004). Perirhinal and postrhinal contributions to remote memory for context. The Journal of neuroscience, 24(49):11023-8.

Bush, G., Luu, P., and Posner, M. I. (2000). Cognitive and emotional influences in anterior cingulate cortex. Trends in cognitive sciences, 4(6):215-222.

Caballero-Bleda, M. and Witter, M. P. (1993). Regional and laminar organization of projections from the presubiculum and parasubiculum to the entorhinal cortex: an anterograde tracing study in the rat. Journal of comparative neurology, 328:115-129.

Cajal, R. (1888). Estructura de los centros nerviosos de la saves. Rev. Trimest. Histol. Norm. Patol., 1:1-10.

Cajal, R. (1893). Estructura del asta de Ammon y fascia dentate. Ann Soc Esp Hist Nat, 22.

Cajal, R. (1909). Histologie du Systeme Nerveux de lHomme et des Vertebres. Maloine, Paris.

Cameron, K. A., Yashar, S., Wilson, C. L., and Fried, I. (2001). Human hippocampal neurons predict how well word pairs will be remembered. Neuron, 30:289-298.

Cammarota, M., Bevilaqua, L. R., Ardenghi, P., Paratcha, G., Levi de Stein, M., Izquierdo, I., and Medina, J. H. (2000). Learning-associated activation of nuclear MAPK, CREB and Elk-1, along with Fos production, in the rat hippocampus after a one-trial avoidance learning: abolition by NMDA receptor blockade. Brain research. Molecular brain research, 76(1):36-46.

Canteras, N. and Swanson, L. W. (1992). Projections of the ventral subiculum to the amygdala, septum, and hypothalamus: a PHAL anterograde tract-tracing study in the rat. Journal of comparative neurology, 324:180-194.

Carlesimo, G. a. (2012). Memory disorders in patients with cerebral tumors. Journal of neuro-oncology, 108(2):253-6.

Carvalho, O. M., Silva, A. J., and Balleine, B. W. (2001). Evidence of Selective Learning Deficits on Tests of $\alpha$-CaMKII T286A Mutant Mice. International Journal of Comparative Psychology, 14:161-174.

Cenquizca, L. a. and Swanson, L. W. (2007). Spatial organization of direct hippocampal field CA1 axonal projections to the rest of the cerebral cortex. Brain research reviews, 56(1):1-26. 
Cermak, L. S. and O'Connor, M. (1983). The anterograde and retrograde retrieval ability of a patient with amnesia due to encephalitis. Neuropsychologia, $21: 213-234$.

Chan, D., Henley, S. M. D., Rossor, M. N., and Warrington, E. K. (2007). Extensive and Temporally Ungraded Retrograde Amnesia in Encephalitis Associated With Antibodies to Voltage-Gated Potassium Channels. Archives of Neurology, 64:404-410.

Chan, S. Y., Murakami, K., and Routtenberg, A. (1986). Phosphoprotein F1: purification and characterization of a brain kinase C substrate related to plasticity. The Journal of neuroscience, 6(12):3618-27.

Chang, F. L. and Greenough, W. T. (1984). Transient and enduring morphological correlates of synaptic activity and efficacy change in the rat hippocampal slice. Brain research, 309(1):35-46.

Cho, Y. H., Beracochea, D., and Jaffard, R. (1993). Extended temporal gradient for the retrograde and anterograde amnesia produced by ibotenate entorhinal cortex lesions in mice. The Journal of neuroscience, 13(4):1759-66.

Cho, Y. H. and Kesner, R. P. (1996). Involvement of entorhinal cortex or parietal cortex in long-term spatial discrimination memory in rats: retrograde amnesia. Behavioral neuroscience, 110(3):436-42.

Churchwell, J. C., Morris, A. M., Musso, N. D., and Kesner, R. P. (2010). Prefrontal and hippocampal contributions to encoding and retrieval of spatial memory. Neurobiology of learning and memory, 93(3):415-21.

Cipolotti, L., Shallice, T., Chan, D., Fox, N., Scahill, R., Harrison, G., Stevens, J., and Rudge, P. (2001). Long-term retrograde amnesia ... the crucial role of the hippocampus. Neuropsychologia, 39:151-172.

Claiborne, B. J., Amaral, D. G., and Cowan, W. M. (1986). A light and electron microscopic analysis of the mossy fibers of the rat dentate gyrus. Journal of comparative neurology, 246:435-458.

Clark, R. E., Broadbent, N. J., and Squire, L. R. (2005a). Hippocampus and remote spatial memory in rats. Hippocampus, 15:260-72.

Clark, R. E., Broadbent, N. J., and Squire, L. R. (2005b). Impaired remote spatial memory after hippocampal lesions despite extensive training beginning early in life. Hippocampus, 15(3):340-346.

Clark, R. E., Broadbent, N. J., and Squire, L. R. (2007). The Hippocampus and Spatial Memory: Findings with a Novel Modification of the Water Maze. Journal of Neuroscience, 27(25):6647-6654. 
Clark, R. E., Broadbent, N. J., Zola, S. M., and Squire, L. R. (2002). Anterograde amnesia and temporally graded retrograde amnesia for a nonspatial memory task after lesions of hippocampus and subiculum. The Journal of neuroscience, 22(11):4663-9.

Coan, E. J., Saywood, W., and Collingridge, G. L. (1987). MK-801 blocks NMDA receptor-mediated synaptic transmission and long term potentiation in rat hippocampal slices. Neuroscience letters, 80:111-4.

Cohen, J. D., Botvinick, M., and Carter, C. S. (2000). Anterior cingulate and prefrontal cortex: whos in control? Nature neuroscience, 3(5):421-423.

Cohen, N. and Eichenbaum, H. (1993). Memory, Amnesia, and the Hippocampal System. MIT Press, Cambridge, MA.

Cohen, N. J. (1984). Preserved learning capacity in amnesia: Evidence for multiple memory systems. In Squire, L. R. and Butters, N., editors, Neuropsychology of memory, pages 83-103. Guilford Press, New York.

Cohen, N. J. and Squire, L. R. (1980). Preserved learning and retention of pattern-analyzing skill in amnesia: Dissociation of knowing how and knowing that. Science, 210:207-210.

Collingridge, B. Y. G. L., Kehl, S. J., Mclennan, H., and Vt, W. (1983). Excitatory amino acids in synaptic transmission in the Schaffer CollateralCommissural pathway of the rat hippocampus. The Journal of Physiology, 334:33-46.

Corbit, L. H. and Balleine, B. W. (2000). The role of the hippocampus in instrumental conditioning. The Journal of neuroscience, 20(11):4233-9.

Corkin, S. (1984). Lasting consequences of bilateral medial temporal lobectomy: Clinical course and experimental findings in H.M. Seminars in Neurology, $4: 249-259$.

Corkin, S. (2002). What's new with the amnesic patient H.M.? Nature reviews. Neuroscience, 3(2):153-60.

Cowan, N. (2001). The magical number 4 in short-term memory: a reconsideration of mental storage capacity. The Behavioral and brain sciences, 24(1):87-114; discussion 114-85.

Damasio, A. R., Eslinger, P. J., Damasio, H., Van Hoesen, G. W., and Cornell, S. (1985). Multimodal amnesic syndrome following bilateral temporal and basal forebrain damage. Archives of neurology, 42(3):252-9.

Davis, H. P. and Squire, L. R. (1984). Protein synthesis and memory: a review. Psychological bulletin, 96(3):518-59. 
De Graan, P. N., Oestreicher, A. B., De Wit, M., Kroef, M., Schrama, L. H., and Gispen, W. H. (1990). Evidence for the binding of calmodulin to endogenous B-50 (GAP-43) in native synaptosomal plasma membranes. Journal of neurochemistry, 55(6):2139-41.

De Roo, M., Klauser, P., and Muller, D. (2008). LTP promotes a selective longterm stabilization and clustering of dendritic spines. PLoS biology, 6(9):e219.

Debiec, J., LeDoux, J. E., and Nader, K. (2002). Cellular and systems reconsolidation in the hippocampus. Neuron, 36(3):527-38.

DeCoteau, W. E., McElvaine, D., Smolentzov, L., and Kesner, R. P. (2009). Effects of rodent prefrontal lesions on object-based, visual scene memory. Neurobiology of learning and memory, 92(4):552-8.

Desmond, N. L. and Levy, W. B. (1983). Synaptic correlates of associative potentiation/depression: an ultrastructural study in the hippocampus. Brain research, 265(1):21-30.

Desmond, N. L. and Levy, W. B. (1986). Changes in the numerical density of synaptic contacts with long-term potentiation in the hippocampal dentate gyrus. The Journal of comparative neurology, 253(4):466-75.

Desmond, N. L. and Levy, W. B. (1988). Synaptic interface surface area increases with long-term potentiation in the hippocampal dentate gyrus. Brain research, 453(1-2):308-14.

Devinsky, O., Morrell, M. J., and Vogt, B. A. (1995). Contributions of anterior cingulate cortex to behaviour. Brain, 118:279-306.

D'Hooge, R. and De Deyn, P. P. (2001). Applications of the Morris water maze in the study of learning and memory. Brain research reviews, 36(1):60-90.

Ding, H. K., Teixeira, C. M., and Frankland, P. W. (2008). Inactivation of the anterior cingulate cortex blocks expression of remote, but not recent , conditioned taste aversion memory. Learning \& Memory, 15:290-293.

Donovan, M. K. and Wyss, J. M. (1983). Evidence for some collateralization between cortical and diencephalic efferent axons of the rat subicular cortex. Brain research, 259:181-192.

Doyere, V., Burette, F., Redini-Del Negron, C., and Laroche, S. (1993). Longterm potentiation of hippocampal afferents and efferents to prefrontal cortex: Implications for associative learning. Neuropsychologia, 31:1031-1053.

Dragunow, M., Currie, R. W., Faull, R. L., Robertson, H. a., and Jansen, K. (1989). Immediate-early genes, kindling and long-term potentiation. Neuroscience and biobehavioral reviews, 13(4):301-13. 
Driscoll, I., Howard, S. R., Prusky, G. T., Rudy, J. W., and Sutherland, R. J. (2005). Seahorse wins all races: hippocampus participates in both linear and non-linear visual discrimination learning. Behavioural brain research, 164(1):29-35.

Dudai, Y. (1996). Consolidation: Fragility on the road to the engram. Neuron, $17: 367-70$.

Dudai, Y. (2000). The shaky trace. Nature, 406(August):686-687.

Dudai, Y. (2004). The neurobiology of consolidations, or, how stable is the engram? Annual review of psychology, 55:51-86.

Dudai, Y. (2009). Predicting not to predict too much: how the cellular machinery of memory anticipates the uncertain future. Philosophical transactions of the Royal Society of London. Series B, Biological sciences, 364(1521):1255-62.

Dudai, Y. (2012). The Restless Engram: Consolidations Never End. Annual Review of Neuroscience, 35(1):227-247.

Dunwiddie, T. and Lynch, G. (1978). Long-term potentiation and depression of synaptic responses in the rat hippocampus: localization and frequency dependency. The Journal of physiology, 276:353-67.

Dupret, D., O'Neill, J., Pleydell-Bouverie, B., and Csicsvari, J. (2010). The reorganization and reactivation of hippocampal maps predict spatial memory performance. Nature neuroscience, 13(8):995-1002.

Ehrlich, Y. H., Rabjohns, R. R., and Routtenberg, A. (1977). Experiential input alters the phosphorylation of specific proteins in brain membranes. Pharmacology, biochemistry, and behavior, 6(2):169-74.

Ehrlich, Y. H. and Routtenberg, A. (1974). Cyclic AMP regulates phosphorylation of three protein components of rat cerebral cortex membranes for thirty minutes. FEBS letters, 45(1):237-43.

Eichenbaum, H., Schoenbaum, G., Young, B., and Bunsey, M. (1996). Functional organization of the hippocampal memory system. Proceedings of the National Academy of Sciences of the United States of America, 93(24):135007 .

Ekstrom, A. D., Kahana, M. J., Caplan, J. B., Fields, T. A., Isham, E. A., Newman, E. L., and Fried, I. (2003). Cellular networks underlying human spatial navigation. Nature, 425:184-188.

Eldridge, L. L., Knowlton, B. J., Furmanski, C. S., Bookheimer, S. Y., and Engel, S. a. (2000). Remembering episodes: a selective role for the hippocampus during retrieval. Nature neuroscience, 3(11):1149-52. 
Epp, J., Keith, J. R., Spanswick, S. C., Stone, J. C., Prusky, G. T., and Sutherland, R. J. (2008). Retrograde amnesia for visual memories after hippocampal damage in rats. Learning \& Memory, 15:214-21.

Epp, J. R., Haack, A. K., and Galea, L. a. M. (2010). Task difficulty in the Morris water task influences the survival of new neurons in the dentate gyrus. Hippocampus, 20(7):866-76.

Etkin, A., Egner, T., and Kalisch, R. (2011). Emotional processing in anterior cingulate and medial prefrontal cortex. Trends in cognitive sciences, 15(2):8593.

Farris, S. M., Robinson, G. E., and Fahrbach, S. E. (2001). Experience- and agerelated outgrowth of intrinsic neurons in the mushroom bodies of the adult worker honeybee. The Journal of neuroscience, 21(16):6395-404.

Feigenbaum, J. D. and Morris, R. G. (2004). Allocentric versus egocentric spatial memory after unilateral temporal lobectomy in humans. Neuropsychology, $18: 462-472$.

Fell, J., Klaver, P., Elfadil, H., Schaller, C., Elger, C. E., and Fernandez, G. (2003). Rhinal-hippocampal theta coherence during declarative memory formation: interaction with gamma synchronization? European Journal of Neuroscience, 17(5):1082-1088.

Ferino, F., Thierry, A. M., and Glowinski, J. (1987). Anatomical and electrophysiological evidence for a direct projection from Ammon's horn to the medial prefrontal cortex in the rat. Experimental brain research, 65(2):421-6.

Fernández, G., Effern, A., Grunwald, T., Pezer, N., Lehnertz, K., Dumpelmann, M., Van Roost, D., and Elger, C. (1999). Real-Time Tracking of Memory Formation in the Human Rhinal Cortex and Hippocampus. Science, 285:1582-1585.

Fernández, G., Klaver, P., Fell, J., Grunwald, T., and Elger, C. E. (2002). Human declarative memory formation: segregating rhinal and hippocampal contributions. Hippocampus, 12(4):514-9.

Frankland, P. W. and Bontempi, B. (2005). The organization of recent and remote memories. Nature reviews. Neuroscience, 6(2):119-30.

Frankland, P. W. and Bontempi, B. (2006). Fast track to the medial prefrontal cortex. Proceedings of the National Academy of Sciences of the United States of America, 103(3):509-510.

Frankland, P. W., Bontempi, B., Talton, L. E., Kaczmarek, L., and Silva, A. J. (2004). The involvement of the anterior cingulate cortex in remote contextual fear memory. Science, 304(5672):881-3. 
Frankland, P. W., O'Brien, C., Ohno, M., Kirkwood, A., and Silva, a. J. (2001). Alpha-CaMKII-dependent plasticity in the cortex is required for permanent memory. Letters to Nature, 411:309-13.

Frey, U., Krug, M., Reymann, K. G., and Matthies, H. (1988). Anisomycin, an inhibitor of protein synthesis, blocks late phases of LTP phenomena in the hippocampal CA1 region in vitro. Brain research, 452(1-2):57-65.

Gaarskjaer, F. B. (1978). Organization of the mossy fiber system of the rat studied in extended hippocampi. II. Experimental analysis of fiber distribution with silver impregnation methods. Journal of comparative neurology, 178:73-88.

Gabrieli, J. D. (1997). Separate Neural Bases of Two Fundamental Memory Processes in the Human Medial Temporal Lobe. Science, 276(5310):264-266.

Gaffan, D. (1974). Recognition impaired and association intact in the memory of monkeys after transection of the fornix. Journal of comparative and physiological psychology, 86(6):1100-9.

Gaffan, D. (1993). Additive effects of forgetting and fornix transfection in the temporal gradient of retrograde amnesia. Neuropsychologia, 31(10):1055-66.

Gafford, G. M., Parsons, R. G., and Helmstetter, F. J. (2013). Memory accuracy predicts hippocampal mTOR pathway activation following retrieval of contextual fear memory. Hippocampus, 23(9):842-7.

Gale, G. D., Anagnostaras, S. G., Godsil, B. P., Mitchell, S., Nozawa, T., Sage, J. R., Wiltgen, B., and Fanselow, M. S. (2004). Role of the basolateral amygdala in the storage of fear memories across the adult lifetime of rats. The Journal of neuroscience, 24(15):3810-5.

Gallagher, M. and Hollandt, P. C. (1992). Preserved Configural Learning and Spatial Learning Impairment in Rats With Hippocampal Damage. Hippocampus, 2(1):81-88.

Ganeshina, O., Berry, R. W., Petralia, R. S., Nicholson, D. A., and Geinisman, Y. (2004). Synapses with a segmented, completely partitioned postsynaptic density express more AMPA receptors than other axospinous synaptic junctions. Neuroscience, 125(3):615-23.

Gaskin, S., Gamliel, A., Tardif, M., Cole, E., and Mumby, D. G. (2009). Incidental (unreinforced) and reinforced spatial learning in rats with ventral and dorsal lesions of the hippocampus. Behavioural Brain Research, 202(1):64-70.

Gaskin, S., Tremblay, A., and Mumby, D. G. (2003). Retrograde and anterograde object recognition in rats with hippocampal lesions. Hippocampus, 13(8):962-9. 
Gehring, W. J. and Knight, R. T. (2000). Prefrontal-cingulate interactions in action monitoring. Nature neuroscience, 3:516-520.

Geinisman, Y., DeToledo-Morrell, L., and Morrell, F. (1991). Induction of longterm potentiation is associated with an increase in the number of axospinous synapses with segmented postsynaptic densities. Brain research, 566(1-2):7788.

Gianotti, C., Nunzi, M. G., Gispen, W. H., and Corradetti, R. (1992). Phosphorylation of the presynaptic protein B-50 (GAP-43) is increased during electrically induced long-term potentiation. Neuron, 8(5):843-8.

Gibb, R. and Kolb, B. (1998). A method for vibratome sectioning of Golgi-Cox stained whole rat brain. Journal of neuroscience methods, 79(1):1-4.

Gilbert, P. E., Kesner, R. P., and Lee, I. (2001). Dissociating Hippocampal Subregions: A Double Dissociation Between Dentate Gyrus and CA1. Hippocampus, 11:626-636.

Giorgi, C., Yeo, G. W., Stone, M. E., Katz, D. B., Burge, C., Turrigiano, G., and Moore, M. J. (2007). The EJC factor eIF4AIII modulates synaptic strength and neuronal protein expression. Cell, 130(1):179-91.

Glaser, E. M. and Loos, H. V. A. N. D. E. R. (1981). Analysis of thick brain sections by obverse-reverse computer microscopy: Application of a new, high clarity Golg-Nissl stain. Journal of neuroscience methods, 4:117-125.

Glenn, M. J., Nesbitt, C., and Mumby, D. G. (2003). Perirhinal cortex lesions produce variable patterns of retrograde amnesia in rats. Behavioural brain research, 141(2):183-93.

Golgi, C. (1873). Sulla struttura della sostanza grigia del cervello. Gazzetta Medica Italiana-Lombardia, 6:244-246.

Gomez, A., Rousset, S., and Charnallet, A. (2012). Spatial Deficits in an Amnesic Patient with Hippocampal Damage : Questioning the Multiple Trace Theory. Hippocampus, 22:1313-1324.

Goodrich-Hunsaker, N. J. and Hopkins, R. O. (2010). Spatial Memory Deficits in a Virtual Radial Arm Maze in Amnesic Participants With Hippocampal Damage. Behavioral Neuroscience, 124(3):405-413.

Gould, E., Beylin, a., Tanapat, P., Reeves, a., and Shors, T. J. (1999). Learning enhances adult neurogenesis in the hippocampal formation. Nature neuroscience, $2(3): 260-5$.

Greenberg, M. E. and Ziff, E. B. (1984). Stimulation of 3T3 cells induces transcription of the c-fos proto-oncogene. Nature, 311(5985):433-8. 
Grimm, R., Schicknick, H., Riede, I., Gundelfinger, E. D., Herdegen, T., Zuschratter, W., and Tischmeyer, W. (1997). Suppression of c-los Induction in Rat Brain Impairs Retention of a Brightness Discrimination Reaction. Learning 83 Memory, 3:402-413.

Groenewegen, H. J., Vermeulen-Van Der Zee, E., Te Kortschot, A., and Witter, M. P. (1987). Organization of the projections from the subiculum to the ventral striatum in the rat: a study using anterograde transport of Phaseolus vulgaris leucoagglutinin. Neuroscience, 23:103-120.

Gundersen, H. J. and Jensen, E. B. (1987). The efficiency of systematic sampling in stereology and its prediction. Journal of microscopy, 147:229-263.

Gundersen, H. J., Jensen, E. B., Kiêu, K., and Nielsen J (1999). The efficiency of systematic sampling in stereology-reconsidered. Journal of microscopy, 193(Pt 3):199-211.

Gusev, P. a., Cui, C., Alkon, D. L., and Gubin, A. N. (2005). Topography of Arc/Arg3.1 mRNA expression in the dorsal and ventral hippocampus induced by recent and remote spatial memory recall: dissociation of CA3 and CA1 activation. The Journal of neuroscience, 25(41):9384-97.

Gusev, P. a. and Gubin, A. N. (2010). Recent and remote memory recalls modulate different sets of stereotypical interlaminar correlations in Arc/Arg3.1 mRNA expression in cortical areas. Brain Research, 1352:118-139.

Guzowski, J. F., Lyford, G. L., Stevenson, G. D., Houston, F. P., McGaugh, J. L., Worley, P. F., and Barnes, C. a. (2000). Inhibition of activity-dependent arc protein expression in the rat hippocampus impairs the maintenance of long-term potentiation and the consolidation of long-term memory. The Journal of neuroscience, 20(11):3993-4001.

Guzowski, J. F., Setlow, B., Wagner, E. K., and McGaugh, J. L. (2001). Experience-dependent gene expression in the rat hippocampus after spatial learning: a comparison of the immediate-early genes Arc, c-fos, and zif268. The Journal of neuroscience, 21(14):5089-98.

Guzowski, J. F., Timlin, J. a., Roysam, B., McNaughton, B. L., Worley, P. F., and Barnes, C. a. (2005). Mapping behaviorally relevant neural circuits with immediate-early gene expression. Current opinion in neurobiology, 15(5):599606 .

Haas, H. L. and Rose, G. (1982). Long-term potentiation of excitatory synaptic transmission in the rat hippocampus: the role of inhibitory processes. The Journal of physiology, 329:541-52.

Haijima, A. and Ichitani, Y. (2008). Anterograde and retrograde amnesia of place discrimination in retrosplenial cortex and hippocampal lesioned rats. Learning $\& 3$ memory, 15(7):477-82. 
Hairston, I. S., Little, M. T. M., Scanlon, M. D., Barakat, M. T., Palmer, T. D., Sapolsky, R. M., and Heller, H. C. (2005). Sleep restriction suppresses neurogenesis induced by hippocampus-dependent learning. Journal of neurophysiology, 94(6):4224-33.

Hamann, S. B. and Squire, L. R. (1997). Intact perceptual memory in the absence of conscious memory. Behavioral Neuroscience, 111(4):850-4.

Hamilton, G. F., Whitcher, L. T., and Klintsova, A. Y. (2010). Postnatal bingelike alcohol exposure decreases dendritic complexity while increasing the density of mature spines in mPFC layer II/III pyramidal neurons. Synapse, 64(2):127-135.

Hampton, R. R., Sherry, D. F., Shettleworth, S. J., Khurgel, M., and Ivy, G. (1995). Hippocampal volume and food-storing behavior are related in parids. Brain, Behavior and Evolution, 45:54-61.

Harand, C., Bertran, F., La Joie, R., Landeau, B., Mézenge, F., Desgranges, B., Peigneux, P., Eustache, F., and Rauchs, G. (2012). The hippocampus remains activated over the long term for the retrieval of truly episodic memories. PloS one, 7(8):e43495.

Harris, K. M., Jensen, F. E., and Tsao, B. (1992). Three-dimensional structure of dendritic spines and synapses in rat hippocampus (CA1) at postnatal day 15 and adult ages: implications for the maturation of synaptic physiology and long-term potentiation. The Journal of neuroscience, 12(7):2685-705.

Hassabis, D., Chu, C., Rees, G., Weiskopf, N., Molyneux, P. D., and Maguire, E. a. (2009). Decoding neuronal ensembles in the human hippocampus. Current biology, 19(7):546-54.

He, J., Yamada, K., Nakajima, A., Kamei, H., and Nabeshima, T. (2002). Learning and memory in two different reward tasks in a radial arm maze in rats. Behavioural Brain Research, 134(1-2):139-48.

Healy, S. D. and Krebs, J. R. (1993). Development of hippocampal specialisation in a food-storing bird. Behavioural Brain Research, 53:127-131.

Hebb, D. O. (1955). Drives and the C.N.S. (conceptual nervous system). The Psychological Review, 62:243-254.

Heindel, C., Salmon, D. P., Shults, W., Walicke, A., and Butters, N. (1989). Neuropsychological evidence for multiple implicit memory systems: A comparison of Alzheimer's, Huntingtons, and Parkinsons disease patients. The Journal of neuroscience, 9:582-587.

Heindel, W. C., Butters, N., and Salmon, D. P. (1988). Impaired learning of a motor skill in patients with huntington's disease. Behavioral neuroscience, 102:141-147. 
Herdegen, T. and Leah, J. D. (1998). Inducible and constitutive transcription factors in the mammalian nervous system: control of gene expression by Jun, Fos and Krox, and CREB/ATF proteins. Brain Research Reviews, 28(3):370490.

Hernandez, a. I., Blace, N., Crary, J. F., Serrano, P. a., Leitges, M., Libien, J. M., Weinstein, G., Tcherapanov, A., and Sacktor, T. C. (2003). Protein kinase $\mathrm{M}$ zeta synthesis from a brain mRNA encoding an independent protein kinase $\mathrm{C}$ zeta catalytic domain. Implications for the molecular mechanism of memory. The Journal of Biological Chemistry, 278(41):40305-16.

Hirano, M. and Noguchi, K. (1998). Dissociation between specific personal episodes and other aspects of remote memory in a patient with hippocampal amnesia. Perceptual and Motor Skills, 87:99-107.

Hirsh, R. (1974). The Hippocampus and contextual retrieval of information from memory: A theory. Behavioral Biology, 12:421-444.

Ho, V., Lee, J., and Martin, K. (2011). The cell biology of synaptic plasticity. Science, 334(6056):623-628.

Hoh, T. E., Kolb, B., Eppel, A., Vanderwolf, C. H., and Cain, D. P. (2003). Role of the neocortex in the water maze task in the rat: a detailed beha $\mathrm{v}$ ioral and Golgi-Cox analysis. Behavioural Brain Research, 138:81-94.

Holahan, M. R., Honegger, K. S., and Routtenberg, A. (2010). Ectopic growth of hippocampal mossy fibers in a mutated GAP-43 transgenic mouse with impaired spatial memory retention. Hippocampus, 20(1):58-64.

Holahan, M. R., Honegger, K. S., Tabatadze, N., and Routtenberg, A. (2007). GAP-43 gene expression regulates information storage. Learning $\& 3$ Memory, 14:407-415.

Holahan, M. R. and Routtenberg, A. (2007). Post-Translational Synaptic Protein Modification as Substrate for Long-Lasting, Remote Memory : An Initial Test. Hippocampus, 97:93-97.

Holahan, M. R. and Routtenberg, A. (2011). Lidocaine Injections Targeting CA3 Hippocampus Impair Long-Term Spatial Memory and Prevent LearningInduced Mossy Fiber Remodeling. Hippocampus, 21(5):532-540.

Holahan, M. R., Westby, E. P., and Albert, K. (2012). Comparison of the MK-801-induced appetitive extinction deficit with pressing for reward and associated pERK1/2 staining in prefrontal cortex and nucleus accumbens. Behavioural brain research, 228(1):194-202.

Holahan, M. R. and White, N. M. (2004). Intra-amygdala muscimol injections impair freezing and place avoidance in aversive contextual conditioning. Learning 83 Memory, 11(4):436-46. 
Holdstock, J. S., Mayes, a. R., Cezayirli, E., Isaac, C. L., Aggleton, J. P., and Roberts, N. (2000). A comparison of egocentric and allocentric spatial memory in a patient with selective hippocampal damage. Neuropsychologia, $38(4): 410-25$.

Holloway, C. M. and McIntyre, C. K. (2011). Post-training disruption of Arc protein expression in the anterior cingulate cortex impairs long-term memory for inhibitory avoidance training. Neurobiology of learning and memory, 95(4):425-32.

Holtmaat, A., Wilbrecht, L., Knott, G. W., Welker, E., and Svoboda, K. (2006). Experience-dependent and cell-type-specific spine growth in the neocortex. Nature, 441(7096):979-83.

Hoover, W. B. and Vertes, R. P. (2007). Anatomical analysis of afferent projections to the medial prefrontal cortex in the rat. Brain Structure 83 Function, 212(2):149-79.

Huang, Y.-Y. and Kandel, E. R. (2005). Theta frequency stimulation induces a local form of late phase LTP in the CA1 region of the hippocampus. Learning 83 memory, 12(6):587-93.

Hübener, M. and Bonhoeffer, T. (2010). Searching for engrams. Neuron, $67(3): 363-71$.

Insausti, R., Herrero, M. T., and Witter, M. P. (1997). Entorhinal cortex of the rat: cytoarchitectonic subdivisions and the origin and distribution of cortical efferents. Hippocampus, 7:146-183.

Isaac, J. T., Oliet, S. H., Hjelmstad, G. O., Nicoll, R. a., and Malenka, R. C. (1996). Expression mechanisms of long-term potentiation in the hippocampus. Journal of Physiology Paris, 90(5-6):299-303.

Ishizuka, N., Weber, I., and Amaral, D. G. (1990). Organization of intrahippocampal projections originating from CA3 pyramidal cells in the rat. Journal of comparative neurology, 295:580-623.

Ito, I., Hidaka, H., and Sugiyama, H. (1991). Effects of KN-62, a specific inhibitor of calcium/calmodulin-dependent protein kinase II, on long-term potentiation in the rat hippocampus. Neuroscience letters, 121(1-2):119-21.

Izquierdo, I., Quillfeldtl, A., Zanatta, M. S., Quevedo, J., Schaeffer, E., Schmitz, P. K., and Medina, J. H. (1997). Sequential role of hippocampus and amygdala, entorhinal cortex and parietal cortex in formation and retrieval of memory for inhibitory avoidance in rats. European Journal of Neuroscience, 9(November 1996):786-793.

Jaffe, D. B. and Gutierrez, R. (2007). Mossy fiber synaptic transmission: communication from the dentate gyrus to area CA3. Progress in brain research, 163:109-132. 
Jan, Y. N. and Jan, L. Y. (2001). Dendrites. Genes $\mathscr{G}$ development, 15(20):262741.

Jarrard, L. E. (1976). Anatomical and behavioral analysis of hippocampal cell fields in rats. Journal of Comparative and Physiological Psychology, 90(11):1035-50.

Jarrard, L. E. (1978). Selective hippocampal lesions: differential effects on performance by rats of a spatial task with preoperative versus postoperative training. Journal of Comparative and Physiological Psychology, 92(6):111927.

Jarrard, L. E. (1983). Selective hippocampal lesions and behavior: Effects of kainic acid lesions on performance of place and cue tasks. Behavioral Neuroscience, 97:873-889.

Jay, T. M., Burette, F., and Laroche, S. (1996). Plasticity of the hippocampalprefrontal cortex synapses. Journal of Physiology Paris, 90:361-366.

Jay, T. M., Glowinski, J., and Thierry, A. M. (1989). Selectivity of the hippocampal projection to the prelimbic area of the prefrontal cortex in the rat. Brain research, 505(2):337-40.

Jay, T. M. and Witter, M. P. (1991). Distribution of hippocampal CA1 and subicular efferents in the prefrontal cortex of the rat studied by means of anterograde transport of Phaseolus vulgaris-leucoagglutinin. The Journal of comparative neurology, 313(4):574-86.

Jeneson, A. and Squire, L. R. (2012). Working memory, long-term memory, and medial temporal lobe function. Learning \&3 Memory, 19(1):15-25.

Johansen, J. P., Cain, C. K., Ostroff, L. E., and LeDoux, J. E. (2011). Molecular mechanisms of fear learning and memory. Cell, 147(3):509-24.

Jones, B. F., Groenewegen, H. J., and Witter, M. P. (2005). Intrinsic connections of the cingulate cortex in the rat suggest the existence of multiple functionally segregated networks. Neuroscience, 133:193-207.

Jones, B. F. and Witter, M. P. (2007). Cingulate Cortex Projections to the Parahippocampal Region and Hippocampal Formation in the Rat. Hippocampus, 17:957-976.

Josselyn, S. (2010). Continuing the search for the engram: examining the mechanism of fear memories. Journal of Psychiatry $\&$ Neuroscience, 35(4):221-228.

Kaczmarek, L. and Kamiska, B. (1989). Molecular biology of cell activation. Experimental Cell Research, 183(1):24-35.

Kandel, E. R. (2001). The molecular biology of memory storage: a dialogue between genes and synapses. Science, 294(5544):1030-8. 
Kandel, E. R. (2012). The molecular biology of memory: cAMP, PKA, CRE, CREB-1, CREB-2, and CPEB. Molecular Brain, 5(1):14.

Kandel, E. R. and Hawkins, R. D. (1992). The biological basis of learning and individuality. Scientific American, 267(3):78-86.

Kapur, N. and Brooks, D. J. (1999). Temporally-specific retrograde amnesia in two cases of discrete bilateral hippocampal pathology. Hippocampus, 9(3):24754 .

Kartsounis, L. D., Rudge, P., and Stevens, J. M. (1995). Bilateral lesions of CAl and CA2 fields of the hippocampus are sufficient to cause a severe amnesic syndrome in humans. Journal of neurology, neurosurgery, and psychiatry, 59:95-98.

Kasai, H., Matsuzaki, M., Noguchi, J., Yasumatsu, N., and Nakahara, H. (2003). Structure-stability-function relationships of dendritic spines. Trends in neurosciences, 26(7):360-8.

Kassardjian, C. D., Tan, Y.-F., Chung, J.-Y. J., Heskin, R., Peterson, M. J., and Broussard, D. M. (2005). The site of a motor memory shifts with consolidation. The Journal of neuroscience, 25(35):7979-85.

Katche, C., Bekinschtein, P., Slipczuk, L., Goldin, A., Izquierdo, I. a., Cammarota, M., and Medina, J. H. (2010). Delayed wave of c-Fos expression in the dorsal hippocampus involved specifically in persistence of long-term memory storage. Proceedings of the National Academy of Sciences of the United States of America, 107(1):349-54.

Kee, N., Teixeira, C. M., Wang, A. H., and Frankland, P. W. (2007). Preferential incorporation of adult-generated granule cells into spatial memory networks in the dentate gyrus. Nature neuroscience, 10(3):355-62.

Kelleher, R. J., Govindarajan, A., and Tonegawa, S. (2004). Translational regulatory mechanisms in persistent forms of synaptic plasticity. Neuron, 44(1):59-73.

Kempermann, G. and Gage, F. H. (2002). Genetic determinants of adult hippocampal neurogenesis correlate with acquisition, but not probe trial performance, in the water maze task. The European journal of neuroscience, 16(1):129-36.

Kentros, C. G., Agnihotri, N. T., Streater, S., Hawkins, R. D., and Kandel, E. R. (2004). Increased Attention to Spatial Context Increases Both Place Field Stability and Spatial Memory. Neuron, 42:283-295.

Kessels, H. W. and Malinow, R. (2009). Synaptic AMPA receptor plasticity and behavior. Neuron, 61(3):340-50. 
Kim, J. J., Clark, R. E., and Thompson, R. F. (1995). Hippocampectomy impairs the memory of recently, but not remotely, acquired trace eyeblink conditioned responses. Behavioral neuroscience, 109(2):195-203.

Kim, J. J. and Fanselow, M. S. (1992). Modality-Specific Retrograde Amnesia of Fear. Science, 256:675-677.

Kirwan, C. B., Bayley, P. J., and Galva, V. V. (2008). Detailed recollection of remote autobiographical. Proceedings of the National Academy of Sciences of the United States of America, 105:2676-2680.

Kirwan, C. B. and Stark, C. E. L. (2004). Medial temporal lobe activation during encoding and retrieval of novel face-name pairs. Hippocampus, 14(7):919-30.

Kitamura, T. and Inokuchi, K. (2014). Role of adult neurogenesis in hippocampal-cortical memory consolidation. Molecular brain, 7(1):13.

Kitamura, T., Saitoh, Y., Takashima, N., Murayama, A., Niibori, Y., Ageta, H., Sekiguchi, M., Sugiyama, H., and Inokuchi, K. (2009). Adult neurogenesis modulates the hippocampus-dependent period of associative fear memory. Cell, 139(4):814-27.

Knafo, S., Ariav, G., Barkai, E., and Libersat, F. (2004). Olfactory learninginduced increase in spine density along the apical dendrites of CA1 hippocampal neurons. Hippocampus, 14(7):819-25.

Koerner, A., Thomas, M. J., Weisand, M. P., and Sutherland, R. J. (1996). Hippocampal-dependent memory consolidation: An evaluation of three hypotheses. In Society for Neuroscience Abstract, page 1118.

Kohler, C. (1985). Intrinsic Projections of the Retrohippocampal Region in the Rat Brain. I. The subicular complex. The journal of compa, 236:504-522.

Kolb, B. and Whishaw, I. Q. (2011). An introduction to brain and behavior. Worth Publishers, New York, N.Y., 3rd edition.

Kopelman, M. D., Lasserson, D., Kingsley, D. R., Bello, F., Rush, C., Stanhope, N., Stevens, T. G., Goodman, G., Buckman, J. R., Heilpern, G., Kendall, B. E., and Colchester, a. C. F. (2003). Retrograde amnesia and the volume of critical brain structures. Hippocampus, 13(8):879-91.

Kopelman, M. D., Stanhope, N., and Kingsley, D. (1999). Retrograde amnesia in patients with diencephalic, temporal lobe or frontal lesions. Neuropsychologia, 37(8):939-58.

Kovács, K. J. (2008). Measurement of immediate-early gene activation- c-fos and beyond. Journal of neuroendocrinology, 20(6):665-72.

Krebs, J. R., Sherry, D. F., Healy, S. D., Perry, V. H., and Vaccarino, a. L. (1989). Hippocampal specialization of food-storing birds. Proceedings of the National Academy of Sciences of the United States of America, 86(4):1388-92. 
Krettek, J. E. and Price, J. L. (1977). Projections from the amygdaloid complex and adjacent olfactory structures to the entorhinal cortex and to the subiculum in the rat and cat. Journal of comparative neurology, 172:723-752.

Krug, M., Lössner, B., and Ott, T. (1984). Anisomycin blocks the late phase of long-term potentiation in the dentate gyrus of freely moving rats. Brain research bulletin, 13(1):39-42.

Kubie, J. L., Sutherland, R. J., and Muller, R. U. (1999). Hippocampal lesions produce a temporally graded retrograde amnesia on a dry version of the Morris swimming task. Psychobiology, 27(3):313-330.

Kuhl, D. and Skehel, P. (1998). Dendritic localization of mRNAs. Current opinion in neurobiology, 8(5):600-6.

Kyd, R. J. and Bilkey, D. K. (2003). Prefrontal Cortex Lesions Modify the Spatial Properties of Hippocampal Place Cells. Cerebral Cortex, 13:444-451.

Lai, C. S. W., Franke, T. F., and Gan, W.-B. (2012). Opposite effects of fear conditioning and extinction on dendritic spine remodelling. Nature, 483(7387):87-91.

Lalonde, R. and Botez, M. I. (1990). The cerebellum and learning processes in animals. Brain research reviews, 15(3):325-32.

Laroche, S. and Davis, S. (2000). Plasticity at Hippocampal to Prefrontal Cortex Synapses: Dual Roles in Working Memory and Consolidation. Hippocampus, 446:438-446.

Lashley, K. S. (1950). In search of the engram. In Danielli, J. F. and Brown, R., editors, Physiological mechanisms in animal behavior, pages 454-482. Academic Press, New York.

Laurent-Demir, C. and Jaffard, R. (1997). Temporally extended retrograde amnesia for spatial information resulting from afterdischarges induced by electrical stimulation of the dorsal hippocampus in mice. Psychobiology, 25(2):133140 .

Lechner, H. A., Squire, L. R., and Byrne, J. H. (1999). 100 Years of Consolidation Remembering Müller and Pilzecker. Learning \& memory, 6:77-87.

Ledoux, J. E. (1993). Emotional Memory: In Search of Systems and Synapses. Annals of the New York Academy of Sciences, 17:149-157.

Lee, A. M., Kanter, B. R., Wang, D., Lim, J. P., Zou, M. E., Qiu, C., Mcmahon, T., Dadgar, J., Fischbach-weiss, S. C., and Messing, R. O. (2013). Prkcz null mice show normal learning and memory. Nature, 493(7432):416-419.

Lee, D. W., Miyasato, L. E., and Clayton, N. S. (1998). Neurobiological bases of spatial learning in the natural environment: neurogenesis and growth in the avian and mammalian hippocampus. Neuroreport, 9(7):R15-27. 
Lee, K., Oliver, M., Schottler, F., and Lynch, G. (1981). Electron microscopic studies of brain slices: The effects of high-frequency stimulation on dendritic ultrastructure. In Kerkut, G. A. and Wheal, H. V., editors, Electrophysiology of Isolated Mammalian CNS Properties, pages 189-211. Academic Press, New York.

Lee, K. S., Schottler, F., Oliver, M., and Lynch, G. (1980). Brief bursts of high-frequency stimulation produce two types of structural change in rat hippocampus. Journal of neurophysiology, 44(2):247-58.

Lehmann, H., Lacanilao, S., and Sutherland, R. J. (2007). Complete or partial hippocampal damage produces equivalent retrograde amnesia for remote contextual fear memories. The European journal of neuroscience, 25(5):1278-86.

Lehmann, H., Rourke, B. K., Booker, A., and Glenn, M. J. (2013). Single session contextual fear conditioning remains dependent on the hippocampus despite an increase in the number of context-shock pairings during learning. Neurobiology of learning and memory, 106:294-9.

Leuner, B., Falduto, J., and Shors, T. J. (2003). Associative memory formation increases the observation of dendritic spines in the hippocampus. The Journal of neuroscience, 23(2):659-65.

Leuner, B., Mendolia-Loffredo, S., Kozorovitskiy, Y., Samburg, D., Gould, E., and Shors, T. J. (2004). Learning enhances the survival of new neurons beyond the time when the hippocampus is required for memory. The Journal of neuroscience, $24(34): 7477-81$.

Li, X.-G., Somogyi, P., Yilnen, A., and Buzsaki, G. (1994). The hippocampal CA3 network: an in vivo intracellular labeling study. Journal of comparative neurology, 339:181-2008.

Ling, D. S. F., Benardo, L. S., and Sacktor, T. C. (2006). Protein kinase Mzeta enhances excitatory synaptic transmission by increasing the number of active postsynaptic AMPA receptors. Hippocampus, 16(5):443-52.

Ling, D. S. F., Benardo, L. S., Serrano, P. a., Blace, N., Kelly, M. T., Crary, J. F., and Sacktor, T. C. (2002). Protein kinase Mzeta is necessary and sufficient for LTP maintenance. Nature neuroscience, 5(4):295-6.

Link, W., Konietzko, U., Kauselmann, G., Krug, M., Schwanke, B., Frey, U., and Kuhl, D. (1995). Somatodendritic expression of an immediate early gene is regulated by synaptic activity. Proceedings of the National Academy of Sciences of the United States of America, 92(12):5734-8.

Liu, Y. C. and Storm, D. R. (1989). Dephosphorylation of neuromodulin by calcineurin. The Journal of biological chemistry, 264(22):12800-4. 
Lø mo, T. (1966). Frequency potentiation of excitatory synaptic activity in the dentate area of the hippocampal formation. Acta Physiol. Scand, 68:Suppl. 277,128 .

Lopez, J., Herbeaux, K., Cosquer, B., Engeln, M., Muller, C., Lazarus, C., Kelche, C., Bontempi, B., Cassel, J.-C., and de Vasconcelos, A. P. (2012). Context-dependent modulation of hippocampal and cortical recruitment during remote spatial memory retrieval. Hippocampus, 22(4):827-41.

Lovinger, D. M., Akers, R. F., Nelson, R. B., Barnes, C. A., McNaughton, B. L., and Routtenberg, A. (1985). A selective increase in phosporylation of protein $\mathrm{F} 1$, a protein kinase $\mathrm{C}$ substrate, directly related to three day growth of long term synaptic enhancement. Brain research, 343(1):137-43.

Lovinger, D. M. and Routtenberg, A. (1988). Synapse-specific protein kinase C activation enhances maintenance of long-term potentiation in rat hippocampus. The Journal of physiology, 400:321-33.

Lyford, G. L., Yamagata, K., Kaufmann, W. E., Barnes, C. a., Sanders, L. K., Copeland, N. G., Gilbert, D. J., Jenkins, N. a., Lanahan, a. a., and Worley, P. F. (1995). Arc, a growth factor and activity-regulated gene, encodes a novel cytoskeleton-associated protein that is enriched in neuronal dendrites. Neuron, 14(2):433-45.

Lynch, G., Larson, J., Kelso, S., Barrionuevo, G., and Schottler, F. (1983). Intracellular injections of EGTA block induction of hippocampal long-term potentiation. Letters to Nature, 305:719-721.

Lynch, M. A., Introduction, I., Erk, B., Potentiation, L.-t., Age, D., and Cognition, E. (2004). Long-Term Potentiation and Memory. Physiological Reviews, $84: 87-136$.

Madroñal, N., Gruart, A., Sacktor, T. C., and Delgado-García, J. M. (2010). PKMzeta inhibition reverses learning-induced increases in hippocampal synaptic strength and memory during trace eyeblink conditioning. PloS one, 5(4):e10400.

Maguire, E. a., Gadian, D. G., Johnsrude, I. S., Good, C. D., Ashburner, J., Frackowiak, R. S., and Frith, C. D. (2000). Navigation-related structural change in the hippocampi of taxi drivers. Proceedings of the National Academy of Sciences of the United States of America, 97(8):4398-403.

Maguire, E. A., Henson, C. A. R. N. A., Mummery, C. J., and Frith, C. D. (2001). Activity in prefrontal cortex, not hippocampus, varies parametrically with the increasing remoteness of memories. Cognitive Neuroscience and Neuropsychology, 12(3):441-444. 
Maier, D. L., Mani, S., Donovan, S. L., Soppet, D., Tessarollo, L., McCasland, J. S., and Meiri, K. F. (1999). Disrupted cortical map and absence of cortical barrels in growth-associated protein (GAP)-43 knockout mice. Proceedings of the National Academy of Sciences of the United States of America, 96(16):9397-402.

Malenka, R. C. and Bear, M. F. (2004). LTP and LTD : An Embarrassment of Riches. Neuron, 44:5-21.

Malenka, R. C., Kauer, J. A., Perkel, D. J., Mauk, M. D., Kelly, P. T., Nicoll, R. A., and Waxham, M. N. (1989). An essential role for postsynaptic calmodulin and protein kinase activity in long-term potentiation. Letters to Nature, 340:554-557.

Malinow, R., Madison, Daniel, V., and Tsien, Richard, W. (1988). Persistent protein kinase acticity underlying long-term potentiation. Letters to Nature, $335: 820-824$.

Malinow, R., Mainen, Z. F., and Hayashi, Y. (2000). LTP mechanisms: from silence to four-lane traffic. Current opinion in neurobiology, 10(3):352-7.

Malinow, R. and Malenka, R. C. (2002). AMPA receptor trafficking and synaptic plasticity. Annual review of neuroscience, 25:103-26.

Malinow, R., Schulman, H., and Tsien, R. (1989). Inhibition of postsynaptic PKC or CaMKII blocks induction but not expression of LTP. Science, 245(4920):862-866.

Manns, J. R., Hopkins, R. O., and Squire, L. R. (2003). Semantic memory and the human hippocampus. Neuron, 38(1):127-33.

Maren, S., Aharonov, G., and Fanselow, M. S. (1997). Neurotoxic lesions of the dorsal hippocampus and Pavlovian fear conditioning in rats. Behavioural brain research, 88(2):261-74.

Marr, D. (1969). A theory of cerebellar cortex. Journal of Physiology, 202:437470.

Marslen-Wilson, W. D. and Teuber, H. L. (1975). Memory for remote events in anterograde amnesia: recognition of public figures from newsphotographs. Neuropsychologia, 13(3):353-64.

Martin, S. J., de Hoz, L., and Morris, R. G. M. (2005). Retrograde amnesia: neither partial nor complete hippocampal lesions in rats result in preferential sparing of remote spatial memory, even after reminding. Neuropsychologia, 43(4):609-24.

Martin, S. J., Grimwood, P. D., and Morris, R. G. M. (2000). Synaptic plasticity and memory: An evaluation of the hypothesis. Annual review of neuroscience, 23:649-711. 
Matsuzaki, M. (2007). Factors critical for the plasticity of dendritic spines and memory storage. Neuroscience research, 57(1):1-9.

Matsuzaki, M., Ellis-Davies, G. C., Nemoto, T., Miyashita, Y., Iino, M., and Kasai, H. (2001). Dendritic spine geometry is critical for AMPA receptor expression in hippocampal CA1 pyramidal neurons. Nature neuroscience, 4(11):1086-92.

Matsuzaki, M., Honkura, N., Ellis-Davies, G. C. R., and Kasai, H. (2004). Structural basis of long-term potentiation in single dendritic spines. Nature, 429(6993):761-6.

Matthies, H., Frey, U., Reymann, K., Krug, M., Jork, R., and Schroeder, H. (1990). Different mechanisms and multiple stages of LTP. Advances in experimental medicine and biology, 268:359-68.

Maviel, T., Durkin, T. P., Menzaghi, F., and Bontempi, B. (2004). Sites of neocortical reorganization critical for remote spatial memory. Science, 305:96-99.

McClelland, J. L., McNaughton, B. L., and O'Reilly, R. C. (1995). Why there are complementary learning systems in the hippocampus and neocortex: insights from the successes and failures of connectionist models of learning and memory. Psychological review, 102(3):419-57.

McDonald, R. J. and White, N. M. (1993). A triple dissociation of memory systems: Hippocampus, amygdala, and dorsal striatum. Behavioral neuroscience, $107(6): 3-22$.

McDonald, R. J. and White, N. M. (1994). Parallel information processing in the water maze: evidence for independent memory systems involving dorsal striatum and hippocampus. Behavioral and neural biology, 61(3):260-70.

McDonald, R. J. and White, N. M. (1995). Hippocampal and nonhippocampal contributions to place learning in rats. Behavioral neuroscience, 109(4):57993.

McGaugh, J., Cahill, L., Ferry, B., and Roozendaal, B. (2000). Brain systems and the regulaton of memory consolidation. In. In Bolhuis, J., editor, Brain, Perception, Memory: Advances in Cognitive Neuroscience, pages 233-251. Oxford University Press, Oxford.

McGaugh, J. L. (2000). Memory-a Century of Consolidation. Science, 287(5451):248-251.

McGaugh, J. L. and Herz, M. J. (1972). Memory Consolidation. Albion, San Fransisco.

McNaughton, B. L. (1982). Long-term synaptic enhancement and short-term potentiation in rat fascia dentata act through different mechanisms. The Journal of physiology, 324:249-62. 
McNaughton, B. L., Barnes, C. A., Gerrard, J. L., Gothard, K., Jung, M. W., Knierim, J. J., Kudrimoti, H., Qin, Y., Skaggs, W. E., Suster, M., and Weaver, K. L. (1996). Deciphering the hippocampal polyglot: the hippocampus as a path integration system. The Journal of experimental biology, 199(Pt 1):17385 .

Meiri, K. F., Saffell, J. L., Walsh, F. S., and Doherty, P. (1998). Neurite outgrowth stimulated by neural cell adhesion molecules requires growthassociated protein-43 (GAP-43) function and is associated with GAP-43 phosphorylation in growth cones. The Journal of neuroscience, 18(24):10429-37.

Messaoudi, E., Kanhema, T., Soulé, J., Tiron, A., Dagyte, G., da Silva, B., and Bramham, C. R. (2007). Sustained Arc/Arg3.1 synthesis controls long-term potentiation consolidation through regulation of local actin polymerization in the dentate gyrus in vivo. The Journal of neuroscience, 27(39):10445-55.

Migues, P. V., Hardt, O., Wu, D. C., Gamache, K., Sacktor, T. C., Wang, Y. T., and Nader, K. (2010). PKMzeta maintains memories by regulating GluR2dependent AMPA receptor trafficking. Nature neuroscience, 13(5):630-4.

Milatovic, D., Montine, T. J., Zaja-milatovic, S., Madison, J. L., Bowman, A. B., and Aschner, M. (2010). Morphometric analysis in neurodegenerative disorders. Current protocols in toxicology, 12:1-14.

Miller, G. A. (1956). The magical number seven, plus or minus two: Some limits on our capacity for processing information. The Psychological Review, $63: 45-59$.

Miller, S. G. and Kennedy, M. B. (1986). Regulation of brain type II $\mathrm{Ca} 2+/$ Calmodulin-dependent protein kinase by autophosphorylation: A Ca2+-triggered molecular switch. Cell, 44:861-870.

Milner, B. (1962). Les troubles de la memoire accompagnant des lesions hippocampiques bilaterales. In Physiologie de lhippocampe, pages 257-272. Centre National de la Recherche Scientifique, Paris.

Milner, B. (1972). Disorders of learning and memory after temporal lobe lesions in man. Clinical Neurosurgery, 19:421-446.

Milner, B., Corkin, S., and Teuber, H.-L. (1968). Further analysis of the hippocampal amnesic syndrome: 14-year follow-up study of H.M. Neuropsychologia, 6(3):215-234.

Milner, P. M. (1957). The cell assembly: mark II. The Psychological Review, 64:242-252.

Mody, I., Baimbridge, K. G., and Miller, J. J. (1984). Blockade of tetanic- and calcium-induced long-term potentiation in the hippocampal slice preparation by neuroleptics. Neuropharmacology, 23(6):625-31. 
Moga, D. E., Calhoun, M. E., Chowdhury, A., Worley, P., Morrison, J. H., and Shapiro, M. L. (2004). Activity-regulated cytoskeletal-associated protein is localized to recently activated excitatory synapses. Neuroscience, 125(1):7-11.

Monaghan, D. T. and Jane, D. E. (2009). Pharmacology of NMDA receptors. In Van Dongen, A. M., editor, Biology of the NMDA Receptor, pages 257-282. CRC Press, Boca Raton.

Montag-Sallaz, M. and Montag, D. (2003). Learning-induced arg 3.1/arc mRNA expression in the mouse brain. Learning \& Memory, 10:99-107.

Morgan, J. I. and Curran, T. (1989). Stimulus-transcription coupling in neurons: role of cellular immediate-early genes. Trends in neurosciences, 12(11):45962 .

Morris, G. M. (1981). Spatial Localization Does Not Require Local Cues the Presence of local cues. Learning and Motivation, 260:239-260.

Morris, R. G. M. (1989). Synaptic Plasticity and Learning : Selective Impairment of Learning in Rats and Blockade of Long-Term Potentiation in viva by the IV-Methyl-D-Aspartate Receptor Antagonist AP5. The Journal of neuroscience, 9(September):3040-3057.

Morris, R. G. M., Anderson, E., Lynch, G. S., and Baudry, M. (1986). Selective impairment of learning and blockade of long-term potentiation by an N-methyl-D-aspartate receptor agonist, AP5. Letters to Nature, 319:774-776.

Morris, R. G. M., Garrud, P., Rawlins, J. N. P., and O'Keefe, J. (1982). Place navigation impaired in rats with hipocampal lesions. Nature, 297:681-683.

Moscovitch, M., Nadel, L., Winocur, G., Gilboa, A., and Rosenbaum, R. S. (2006). The cognitive neuroscience of remote episodic, semantic and spatial memory. Current opinion in neurobiology, 16(2):179-90.

Moscovitch, M., Rosenbaum, R. S., Gilboa, A., Addis, D. R., Westmacott, R., Grady, C., McAndrews, M. P., Levine, B., Black, S., Winocur, G., and Nadel, L. (2005). Functional neuroanatomy of remote episodic, semantic and spatial memory: a unified account based on multiple trace theory. Journal of anatomy, 207(1):35-66.

Moser, E., Moser, M. B., and Andersen, P. (1993). Spatial learning impairment parallels the magnitude of dorsal hippocampal lesions, but is hardly present following ventral lesions. The Journal of neuroscience, 13(9):3916-25.

Moser, M. B., Moser, E. I., Forrest, E., Andersen, P., and Morris, R. G. (1995). Spatial learning with a minislab in the dorsal hippocampus. Proceedings of the National Academy of Sciences of the United States of America, 92(21):9697701. 
Müller, G. E. and Pilzecker, A. (1900). Experimentelle beitrage zur lehre von gedachtnis. Zeitschrift Psychologie, pages 1-300.

Muller, G. E. and Pilzecker, A. (1900). Experimentelle beitrage zur lehre von gedachtnis. Z. Psychol., 1:1-300.

Mumby, D. G., Astur, R. S., Weisend, M. P., and Sutherland, R. J. (1999). Retrograde amnesia and selective damage to the hippocampal formation: memory for places and object discriminations. Behavioural brain research, 106(1-2):97-107.

Mumby, D. G. and Glenn, M. J. (2000). Anterograde and retrograde memory for object discriminations and places in rats with perirhinal cortex lesions. Behavioural brain research, 114(1-2):119-34.

Murmu, M. S., Salomon, S., Biala, Y., Weinstock, M., Braun, K., and Bock, J. (2006). Changes of spine density and dendritic complexity in the prefrontal cortex in offspring of mothers exposed to stress during pregnancy. The European journal of neuroscience, 24(5):1477-87.

Naber, P. A., da Silva, F. H. L., and Witter, M. P. (2001). Reciprocal connections between the entorhinal cortex and hippocampal fields CA1 and the subiculum are in register with the projections from CA1 to the subiculum. Hippocampus, 11:99-104.

Nadel, L. and Bohbot, V. (2001). Consolidation of memory. Hippocampus, 11(1):56-60.

Nadel, L. and Hardt, O. (2004). The spatial brain. Neuropsychology, 18(3):4736.

Nadel, L. and Moscovitch, M. (1997). Memory consolidation, retrograde amnesia and the hippocampal complex. Current opinion in neurobiology, 7(2):217-27.

Nadel, L. and Moscovitch, M. (1998). Hippocampal contributions to cortical plasticity. Neuropharmacology, 37(4-5):431-9.

Nadel, L., Winocur, G., and Ryan, L. (2007). Systems consolidation and hippocampus : two views. Debates in Neuroscience, 1:55-66.

Nelson, R. B., Linden, D. J., Hyman, C., Pfenninger, K. H., and Routtenberg, A. (1989). The two major phosphoproteins in growth cones are probably identical to two protein kinase $\mathrm{C}$ substrates correlated with persistence of long-term potentiation. The Journal of neuroscience, 9(2):381-9.

Nielander, H. B., Schrama, L. H., van Rozen, A. J., Kasperaitis, M., Oestreicher, A. B., Gispen, W. H., and Schotman, P. (1990). Mutation of serine 41 in the neuron-specific protein B-50 (GAP-43) prohibits phosphorylation by protein kinase C. Journal of neurochemistry, 55(4):1442-5. 
Noulhiane, M., Piolino, P., Hasboun, D., Clemenceau, S., Baulac, M., Samson, S., Upr, L. C., Marie, P., and Pitie, C. H. U. (2007). Autobiographical memory after temporal lobe resection : neuropsychological and MRI volumetric findings. Brain, 130:3184-3199.

O'Connor, M., Butters, N., Miliotis, P., Eslinger, P., and Cermak, L. S. (1992). The dissociation of anterograde and retrograde amnesia in a patient with herpes encephalitis. Journal of clinical and experimental neuropsychology, 14(2):159-78.

Okamoto, K.-I., Nagai, T., Miyawaki, A., and Hayashi, Y. (2004). Rapid and persistent modulation of actin dynamics regulates postsynaptic reorganization underlying bidirectional plasticity. Nature neuroscience, 7(10):1104-12.

O'Keefe, J. and Nadel, L. (1978). The hippocampus as a cognitive map. Oxford University Press, Oxford.

Olton, D. S. and Papas, B. C. (1979). Spatial memory and hippocampal function. Neuropsychologia, 17:669-682.

Olton, D. S. and Samuelson, R. J. (1976). Remembrance of places passed: spatial memory in rats. Journal of experimental psychology. Animal behavior processes, 2:97-116.

Olton, D. S., Walker, J. A., and Gage, F. H. (1978). Hippocampal connections and spatial discrimination. Brain research, 139:295-308.

Öngür, D. and Price, J. L. (2000). The Organization of Networks within the Orbital and Medial Prefrontal Cortex of Rats, Monkeys and Humans. Cerebral Cortex, 10:206-219.

Oswald, B. B., Maddox, S. A., Tisdale, N., and Powell, D. A. (2010). Neurobiology of Learning and Memory Encoding and retrieval are differentially processed by the anterior cingulate and prelimbic cortices : A study based on trace eyeblink conditioning in the rabbit. Neurobiology of Learning and Memory, 93(1):37-45.

Otani, S., Marshall, C. J., Tate, W. P., Goddard, G. V., and Abraham, W. C. (1989). Maintenance of long-term potentiation in rat dentate gyrus requires protein synthesis but not messenger RNA synthesis immediately posttetanization. Neuroscience, 28(3):519-26.

Packard, M. G. (2009). Exhumed from thought: basal ganglia and response learning in the plus-maze. Behavioural brain research, 199(1):24-31.

Packard, M. G., Hirsh, R., and White, M. (1989). Differential effects of fornix and caudate nucleus lesions on two radial maze tasks: Evidence for multiple memory systems. The Journal of neuroscience, 9:1465-1472. 
Paller, K. a. and Wagner, A. D. (2002). Observing the transformation of experience into memory. Trends in cognitive sciences, 6(2):93-102.

Pannese, E. (2010). The Golgi Stain: Invention, diffusion and impact on neurosciences. Journal of the History of the Neurosciences Basic and Clinical Perspectives, 8:132-140.

Papa, M., Pellicano, M. P., Cerbone, A., Lamberti-D’Mello, C., Menna, T., Buono, C., Giuditta, A., Welzl, H., and Sadile, A. G. (1995). Immediate early genes and brain DNA remodeling in the Naples high- and low-excitability rat lines following exposure to a spatial novelty. Brain research bulletin, $37(2): 111-8$.

Papez, J. W. (1937). A proposed mechanism of emotion. Archives of Neurology And Psychiatry, 38:725.

Parslow, D. M., Rose, D., Brooks, B., Fleminger, S., Gray, J. A., Giampietro, V., Brammer, M. J., Williams, S., Gasston, D., Andrew, C., Vythelingum, G. N., Loannou, G., Simmons, A., and Morris, R. G. (2004). Allocentric spatial memory activation of the hippocampal formation measured with fMRI. Neuropsychology, 18:450-461.

Parsons, R. G. and Davis, M. (2011). Temporary disruption of fear potentiated startle following PKMZ inhibition in the amygdala. Nature neuroscience, 14(3):295-296.

Pascale, A., Gusev, P. A., Amadio, M., Dottorini, T., Govoni, S., Alkon, D. L., and Quattrone, A. (2004). Increase of the RNA-binding protein $\mathrm{HuD}$ and posttranscriptional up-regulation of the GAP-43 gene during spatial memory. Proceedings of the National Academy of Sciences of the United States of America, 101(5):1217-1222.

Pastalkova, E., Serrano, P., Pinkhasova, D., Wallace, E., Fenton, A. A., and Sacktor, T. C. (2006). Storage of spatial information by the maintenance mechanism of LTP. Science, 313(5790):1141-4.

Paxinos, G. and Watson, C. (2006). The Rat Brain in Stereotaxic Coordinates, volume 170. Academic Press, San Diego, sixth edition.

Paylor, R., Johnson, R. S., Papaioannou, V., Spiegelman, B. M., and Wehner, J. M. (1994). Behavioral assessment of c-fos mutant mice. Brain research, 651:275-282.

Pikkarainen, M., Ronkko, S., Savander, V., Insausti, R., and Pitkanen, A. (1999). Projections from the lateral, basal, and accessory basal nuclei of the amygdala to the hippocampal formation in rat. Journal of comparative neurology, 403:229-260. 
Pitkanen, A., Pikkarainen, M., Nurminen, N., and Ylinen, A. (2000). Reciprocal connections between the amygdala and the hippocampal formation, perirhinal cortex and postrhinal cortex in the rat. Annals of the New York Academy of Sciences, 911:369-391.

Plath, N., Ohana, O., Schmitz, D., Gross, C., Mao, X., Engelsberg, A., Mahlke, C., Welzl, H., Kobalz, U., Stawrakakis, A., Fernandez, E., Waltereit, R., Bicksander, A., Therstappen, E., Cooke, S. F., Blanquet, V., Wurst, W., Salmen, B., Bo, M. R., Grant, S. G. N., Bliss, T. V. P., Wolfer, D. P., and Kuhl, D. (2006). Arc / Arg3 . 1 Is Essential for the Consolidation of Synaptic Plasticity and Memories. Neuron, 52:437-444.

Poldrack, R. a. and Packard, M. G. (2003). Competition among multiple memory systems: converging evidence from animal and human brain studies. Neuropsychologia, 41(3):245-51.

Posner, M. I. and Keele, S. W. (1968). On the genesis of abstract ideas. Experimental Psychology, 77:353-363.

Quillfeldt, J. A., Zanatta, M. S., Schmitz, P. K., Quevedo, J., Schaeffer, E., Lima, J. B., Medina, J. H., and Izquierdo, I. (1996). Different brain areas are involved in memory expression at different times from training. Neurobiology of learning and memory, 66(2):97-101.

Quinn, J. J., Ma, Q. D., Tinsley, M. R., Koch, C., and Fanselow, M. S. (2008). Inverse temporal contributions of the dorsal hippocampus and medial prefrontal cortex to the expression of long-term fear memories. Learning $\&$ memory, 15(5):368-72.

Radley, J. J., Sisti, H. M., Hao, J., Rocher, a. B., McCall, T., Hof, P. R., McEwen, B. S., and Morrison, J. H. (2004). Chronic behavioral stress induces apical dendritic reorganization in pyramidal neurons of the medial prefrontal cortex. Neuroscience, 125(1):1-6.

Ramos, J. M. J. (1998). Retrograde amnesia for spatial information : a dissociation between intra and extramaze cues following hippocampus lesions in rats. European Journal of Neuroscience, 10(July):3295-3301.

Ramos, J. M. J. (2008). Hippocampal damage impairs long-term spatial memory in rats: comparison between electrolytic and neurotoxic lesions. Physiology \& behavior, 93(4-5):1078-85.

Ramos, J. M. J. (2009). Remote spatial memory and the hippocampus: effect of early and extensive training in the radial maze. Learning 83 memory, 16(9):554-63.

Ranjan, A. and Mallick, B. N. (2010). A modified method for consistent and reliable Golgi-cox staining in significantly reduced time. Frontiers in neurology, 1(December):157. 
Rapanelli, M., Frick, L. R., and Zanutto, B. S. (2011). Learning an operant conditioning task differentially induces gliogenesis in the medial prefrontal cortex and neurogenesis in the hippocampus. PloS one, 6(2):e14713.

Raymond, C. R. (2007). LTP forms 1, 2 and 3: different mechanisms for the "long" in long-term potentiation. Trends in neurosciences, 30(4):167-75.

Reber, P. J., Stark, C. E. L., and Squire, L. R. (1998). Contrasting Cortical Activity Associated with Category Memory and Recognition Memory. Learning E5 Memory, 5:420-428.

Reed, J. M. and Squire, L. R. (1998). Retrograde amnesia for facts and events: findings from four new cases. The Journal of neuroscience, 18(10):3943-54.

Remondes, M. and Schuman, E. M. (2004). Role for a cortical input to hippocampal area CA1 in the consolidation of a long-term memory. Letters to Nature, 431:699-703.

Rempel-Clower, N. L., Zola, S. M., Squire, L. R., and Amaral, D. G. (1996). Three cases of enduring memory impairment after bilateral damage limited to the hippocampal formation. The Journal of neuroscience, 16(16):5233-55.

Restivo, L., Roman, F. S., Ammassari-Teule, M., and Marchetti, E. (2006). Simultaneous olfactory discrimination elicits a strain-specific increase in dendritic spines in the hippocampus of inbred mice. Hippocampus, 16(5):472-9.

Restivo, L., Vetere, G., Bontempi, B., and Ammassari-Teule, M. (2009). The formation of recent and remote memory is associated with time-dependent formation of dendritic spines in the hippocampus and anterior cingulate cortex. The Journal of neuroscience, 29(25):8206-14.

Reymann, K. G., Brdemann, R., Kase, H., and Matthies, H. (1988). Inhibitors of calmodulin and protein kinase $\mathrm{C}$ block different phases of hippocampal long-term potentiation. Brain research, 461:388-392.

Ribot, T. (1882). Diseases of the memory: An essay in the positive psychology. Appleton and Company, New York, N.Y.

Riedel, G., Micheau, J., Lam, A. G. M., Roloff, E. L., Martin, S. J., Bridge, H., and Hoz, L. D. (1999). Reversible neural inactivation reveals hippocampal participation in several memory processes. Nature neuroscience, 2(10):898905.

Roberson, E. D. and Sweatt, J. D. (1999). A Biochemical Blueprint for LongTerm Memory. Learning $\& 3$ Memory, 6:381-388.

Rodríguez, J. J., Davies, H. A., Silva, A. T., De Souza, I. E. J., Peddie, C. J., Colyer, F. M., Lancashire, C. L., Fine, A., Errington, M. L., Bliss, T. V. P., and Stewart, M. G. (2005). Long-term potentiation in the rat dentate gyrus is associated with enhanced Arc/Arg3.1 protein expression in spines, dendrites and glia. The European journal of neuroscience, 21(9):2384-96. 
Ron, S., Dudai, Y., and Segal, M. (2012). Overexpression of PKM $\zeta$ alters morphology and function of dendritic spines in cultured cortical neurons. Cerebral cortex, 22(11):2519-28.

Rosenbaum, R. S., Gao, F., Richards, B., Black, S. E., and Moscovitch, M. (2005). "Where to?" remote memory for spatial relations and landmark identity in former taxi drivers with Alzheimer's disease and encephalitis. Journal of cognitive neuroscience, 17(3):446-62.

Rosenbaum, R. S., Moscovitch, M., Foster, J. K., Schnyer, D. M., Gao, F., Kovacevic, N., Verfaellie, M., Black, S. E., and Levine, B. (2008). Patterns of Autobiographical Memory Loss in Medial-Temporal Lobe Amnesic Patients. Journal of cognitive neuroscience, 20:1490-1506.

Rosenbaum, R. S., Priselac, S., Köhler, S., Black, S. E., Gao, F., Nadel, L., and Moscovitch, M. (2000). Remote spatial memory in an amnesic person with extensive bilateral hippocampal lesions. Nature neuroscience, 3(10):1044-8.

Rosenbaum, R. S., Winocur, G., and Moscovitch, M. (2001). New views on old memories: re-evaluating the role of the hippocampal complex. Behavioural brain research, 127(1-2):183-97.

Ross, R. S. and Eichenbaum, H. (2006). Dynamics of hippocampal and cortical activation during consolidation of a nonspatial memory. The Journal of neuroscience, 26(18):4852-9.

Routtenberg, A., Cantallops, I., Zaffuto, S., Serrano, P., and Namgung, U. (2000). Enhanced learning after genetic overexpression of a brain growth protein. Proceedings of the National Academy of Sciences of the United States of America, 97(13):7657-7662.

Routtenberg, A. and Ehrlich, Y. H. (1975). Endogenous phosphorylation of four cerebral cortical membrane proteins: role of cyclic nucleotides, ATP and divalent cations. Brain research, 92(3):415-30.

Rowland, D. and Kentros, C. (2008). Potential Anatomical Basis for Attentional Modulation of Hippocampal Neurons. Annals of the New York Academy of Sciences, 1129:213-224.

Roxin, A. and Fusi, S. (2013). Efficient Partitioning of Memory Systems and Its Importance for Memory Consolidation. PloS one, 9(7):e1003146.

Rudy, J. W., Biedenkapp, J. C., and O'Reilly, R. C. (2005). Prefrontal cortex and the organization of recent and remote memories: an alternative view. Learning $\&$ memory, 12(5):445-6.

Sacktor, T. C. (2008). PKMzeta, LTP maintenance, and the dynamic molecular biology of memory storage. Progress in brain research, 169(07):27-40. 
Sacktor, T. C. (2011). How does PKM $\zeta$ maintain long-term memory? Nature reviews. Neuroscience, 12(1):9-15.

Saint-Cyr, J. A., Taylor, A. E., and Lang, A. E. (1988). Procedural learning and neostriatal dysfunction in man. Brain, 111:941-959.

Salmon, D. P., Zola-Morgan, S., and Squire, L. R. (1987). Retrograde amnesia following combined hippocampus-amygdala lesions in monkeys. Psychobiology, 15(1):37-47.

Sanders, H. I. and Warrington, E. K. (1971). Memory for remote events in amnesic patients. Brain, 94(4):661-8.

Schacter, D. L. (1992). Review Implicit knowledge : New perspectives on unconscious processes. Proceedings of the National Academy of Sciences of the United States of America, 89:11113-11117.

Schaffer, K. (1892). Beitrag zur Histologie der Ammonshornformation. Arch mikroskop Anatomie, 39:611-632.

Schlesiger, M. I., Cressey, J. C., Boublil, B., Koenig, J., Melvin, N. R., Leutgeb, J. K., and Leutgeb, S. (2013). Neurobiology of Learning and Memory Hippocampal activation during the recall of remote spatial memories in radial maze tasks. Neurobiology of Learning and Memory, 106:324-333.

Schott, B. H., Henson, R. N., Richardson-Klavehn, A., Becker, C., Thoma, V., Heinze, H.-J., and Düzel, E. (2005). Redefining implicit and explicit memory: the functional neuroanatomy of priming, remembering, and control of retrieval. Proceedings of the National Academy of Sciences of the United States of America, 102(4):1257-62.

Scorcioni, R., Lazarewicz, M. T., and Ascoli, G. a. (2004). Quantitative morphometry of hippocampal pyramidal cells: differences between anatomical classes and reconstructing laboratories. The Journal of comparative neurology, 473(2):177-93.

Scoville, W. B. and Milner, B. (1957). Loss of Recent Memory After Bilateral Hippocampal Lesions. Journal of neurology, neurosurgery, and psychiatry, 20:11-21.

Serrano, P., Friedman, E. L., Kenney, J., Taubenfeld, S. M., Zimmerman, J. M., Hanna, J., Alberini, C., Kelley, A. E., Maren, S., Rudy, J. W., Yin, J. C. P., Sacktor, T. C., and Fenton, A. a. (2008). PKMzeta maintains spatial, instrumental, and classically conditioned long-term memories. PLoS biology, 6(12):2698-706.

Serrano, P., Yao, Y., and Sacktor, T. C. (2005). Persistent phosphorylation by protein kinase Mzeta maintains late-phase long-term potentiation. The Journal of neuroscience, 25(8):1979-84. 
Shao, C. Y., Sondhi, R., van de Nes, P. S., and Sacktor, T. C. (2011). PKM $\zeta$ is necessary and sufficient for synaptic clustering of PSD-95. Hippocampus, $22(7): 1501-7$.

Shema, R., Sacktor, T. C., and Dudai, Y. (2007). Rapid erasure of long-term memory associations in the cortex by an inhibitor of PKM zeta. Science, 317(5840):951-3.

Shepherd, J. D. and Bear, M. F. (2011). New views of Arc, a master regulator of synaptic plasticity. Nature neuroscience, 14(3):279-84.

Shepherd, J. D., Rumbaugh, G., Wu, J., Chowdhury, S., Kuhl, D., Huganir, R. L., and Worley, P. F. (2006). Arc mediates homeostatic synaptic scaling of AMPA receptors. Neuron, 52(3):475-484.

Sherry, D. F., Vaccarino, A. L., Buckenham, K., and Herz, R. S. (1989). The hippocampal complex of food-storing birds. Brain, Behavior and Evolution, $34: 308-317$.

Shibata, H. and Naito, J. (2008). Organization of Anterior Cingulate and Frontal Cortical Projections to the Retrosplenial Cortex in the Rat. The Journal of comparative neurology, 45:30-45.

Shimizu, E., Tang, Y. P., Rampon, C., and Tsien, J. Z. (2000). NMDA receptordependent synaptic reinforcement as a crucial process for memory consolidation. Science, 290(5494):1170-4.

Shull, R. N. and Holloway, F. a. (1985). Behavioral effects of hippocampal system lesions on rats in an operant paradigm. Brain research bulletin, 14(4):31522 .

Skene, J. H. (1989). Axonal growth-associated proteins. Annual review of neuroscience, $12: 127-56$.

Skinner, B. F. (1937). Two types of conditioned reflex: a reply to Konorski and Miller. J. Gen. Psychol., 16:272-279.

Söderlund, H., Moscovitch, M., Kumar, N., Mandic, M., and Levine, B. (2012). As time goes by: Hippocampal connectivity changes with remoteness of autobiographical memory retrieval. Hippocampus, 22(4):670-679.

Son, H., Davis, P. J., and Carpenter, D. O. (1997). Time Course and Involvement of Protein Kinase C-Mediated Phosphorylation of Fl / GAP-43 in Area CA3 After Mossy Fiber Stimulation. Cellular and molecular neurobiology, $17(2): 171-194$.

Spacek, J. (1989). Dynamics of the Golgi method: a time-lapse study of the early stages of impregnation in single sections. Journal of neurocytology, 18(1):27-38. 
Sparks, F. T., Lehmann, H., Hernandez, K., and Sutherland, R. J. (2011). Suppression of neurotoxic lesion-induced seizure activity: evidence for a permanent role for the hippocampus in contextual memory. PloS one, 6(11):e27426.

Squire, L. R. (1982). The neuropsychology of human memory. Annual review of neuroscience, 5:241-273.

Squire, L. R. (1992). Memory and the hippocampus: a synthesis from findings with rats, monkeys, and humans. Psychological review, 99(2):195-231.

Squire, L. R. (2004). Memory systems of the brain: a brief history and current perspective. Neurobiology of learning and memory, 82(3):171-7.

Squire, L. R. and Alvarez, P. (1995). Retrograde amnesia and memory consolidation: a neurobiological perspective. Current opinion in neurobiology, $5(2): 169-77$.

Squire, L. R. and Barondes, S. H. (1972). Variable decay of memory and its recovery in cycloheximide-treated mice. Proceedings of the National Academy of Sciences of the United States of America, 69(6):1416-20.

Squire, L. R., Clark, R. E., and Bayley, P. J. (2004). Medial temporal lobe function and memory. In Gazzaniga, M. S., editor, The Cognitive Neurosciences, pages 691-708. MIT Press, USA, 3 edition.

Squire, L. R., Clark, R. E., and Knowlton, B. J. (2001). Retrograde amnesia. Hippocampus, 11(1):50-5.

Squire, L. R. and Kandel, E. R. (1999). Memory: From mind to molecules. W.H. Freeman and Co, New York.

Stark, C. E. and Okado, Y. (2003). Making memories without trying: medial temporal lobe activity associated with incidental memory formation. Journal of Neuroscience, 23:6748-6753.

Stark, C. E. and Squire, L. R. (2000). Functional magnetic resonance imaging (fMRI) activity in the hippocampal region during recognition memory. The Journal of neuroscience, 20(20):7776-81.

Stark, C. E. and Squire, L. R. (2001). Simple and associative recognition mem-

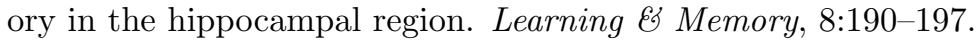

Steinvorth, S., Levine, B., and Corkin, S. (2005). Medial temporal lobe structures are needed to re-experience remote autobiographical memories : evidence from H. M . and W . R . Neuropsychologia, 43:479-496.

Steward, O., Wallace, C. S., Lyford, G. L., and Worley, P. F. (1998). Synaptic activation causes the mRNA for the IEG Arc to localize selectively near activated postsynaptic sites on dendrites. Neuron, 21(4):741-51. 
Steward, O. and Worley, P. F. (2001). Selective Targeting of Newly Synthesized Arc mRNA to Active Synapses Requires NMDA Receptor Activation University of California at Irvine. Neuron, 30:227-240.

Strittmatter, S. M., Fankhauser, C., Huang, P. L., Mashimo, H., and Fishman, M. C. (1995). Neuronal pathfinding is abnormal in mice lacking the neuronal growth cone protein GAP-43. Cell, 80(3):445-52.

Sutherland, R. J. and Lehmann, H. (2011). Alternative conceptions of memory consolidation and the role of the hippocampus at the systems level in rodents. Current opinion in neurobiology, 21(3):446-51.

Sutherland, R. J., O'Brien, J., and Lehmann, H. (2008). Absence of systems consolidation of fear memories after dorsal, ventral, or complete hippocampal damage. Hippocampus, 18(7):710-718.

Sutherland, R. J., Sparks, F. T., and Lehmann, H. (2010). Hippocampus and retrograde amnesia in the rat model: a modest proposal for the situation of systems consolidation. Neuropsychologia, 48(8):2357-69.

Sutherland, R. J., Weisend, M. P., Mumby, D., Astur, R. S., Hanlon, F. M., Koerner, a., Thomas, M. J., Wu, Y., Moses, S. N., Cole, C., Hamilton, D. a., and Hoesing, J. M. (2001). Retrograde amnesia after hippocampal damage: recent vs. remote memories in two tasks. Hippocampus, 11(1):27-42.

Sutherland, R. J., Whishaw, I. Q., and Kolb, B. (1983). A Behavioural Analysis of Spatial Localization Following Electrolytic, Kainate- or Colchicine-Induced Damage to the Hippocampal Formation in the Rat. Behavioural brain research, 7:133-153.

Swanson, L. W. and Cowan, W. M. (1975). Hippocampo-hypothalamic connections: origin in subicular cortex, not Ammons horn. Science, 189:303-304.

Swanson, L. W., Sawchenko, P. E., and Cowan, W. M. (1981). Evidence for collateral projections by neurons in ammons horn, the dentate gyrus, and the subiculum: a multiple retrograde labeling study in the rat. Journal of Neuroscience, 1:548-559.

Swanson, L. W., Wyss, J. M., and Cowan, W. M. (1978). An autoradiographic study of the organization of intrahippocampal association pathways in the rat. Journal of comparative neurology, 181:681-716.

Sweatt, J. D. (1999). Toward a Molecular Explanation for Long-Term Potentiation. Learning \& Memory, 6(5):399-416.

Sweatt, J. D. (2010). Mechanisms of memory. Academic Press, London, 2 edition. 
Takashima, A., Nieuwenhuis, I. L. C., Jensen, O., Talamini, L. M., Rijpkema, M., and Fernández, G. (2009). Shift from hippocampal to neocortical centered retrieval network with consolidation. The Journal of neuroscience, 29(32):10087-93.

Takashima, A., Nieuwenhuis, I. L. C., Rijpkema, M., Petersson, K. M., Jensen, O., and Fernández, G. (2007). Memory trace stabilization leads to large-scale changes in the retrieval network : A functional MRI study on associative memory. Learning \& Memory, 14:472-479.

Takashima, A., Petersson, K. M., Rutters, F., Tendolkar, I., Jensen, O., Zwarts, M. J., and Mcnaughton, B. L. (2006). Declarative memory consolidation in humans : A prospective functional magnetic resonance. Proceedings of the National Academy of Sciences of the United States of America, 103(3):756761.

Takehara, K., Kawahara, S., and Kirino, Y. (2003). Time-dependent reorganization of the brain components underlying memory retention in trace eyeblink conditioning. The Journal of neuroscience, 23(30):9897-905.

Takehara, K., Kawahara, S., Takatsuki, K., and Kirino, Y. (2002). Time-limited role of the hippocampus in the memory for trace eyeblink conditioning in mice. Brain research, 951(2):183-90.

Tamamaki, N., Abe, K., and Nojyo, Y. (1987). Columnar organization in the subiculum formed by axon branches originating from single CA1 pyramidal neurons in the rat hippocampus. Brain research, 412:156-160.

Teixeira, C. M., Pomedli, S. R., Maei, H. R., Kee, N., and Frankland, P. W. (2006). Involvement of the anterior cingulate cortex in the expression of remote spatial memory. The Journal of neuroscience, 26(29):7555-64.

Teng, E. and Squire, L. R. (1999). Memory for places learned long ago is intact after hippocampal damage. Nature, 400(6745):675-7.

Thomas, A. G., Koumellis, P., and Dineen, R. a. (2011). The fornix in health and disease: an imaging review. Radiographics, 31(4):1107-21.

Thompson, R. F. (1986). The neurobiology of learning and memory. Science, 233:941-947.

Thomsen, M. S., Mikkelsen, J. D., Timmermann, D. B., Peters, D., Martens, H., and Hansen, H. H. (2008). The selective alpha7 nicotinic acetylcholine receptor agonist A-582941 activates immediate early genes in limbic regions of the forebrain: Differential effects in the juvenile and adult rat. Neuroscience, 154:741-753.

Thornton, J. A., Rothblat, L. A., and Murray, E. A. (1997). Rhinal cortex removal produces amnesia for preoperatively learned discrimination problems but fails to disrupt postoperative acquisition and retention in rhesus monkeys. The Journal of neuroscience, 17(21):8536-49. 
Tischmeyer, W. and Grimm, R. (1999). Activation of immediate early genes and memory formation. Cellular and molecular life sciences, 55(4):564-74.

Trogrlic, L., Wilson, Y. M., Newman, A. G., and Murphy, M. (2011). Context fear learning specifically activates distinct populations of neurons in amygdala and hypothalamus. Learning $\mathscr{E}$ memory, 18(10):678-87.

Trommald, M., Hulleberg, G., and Andersen, P. (1996). Long-term Potentiation Is Associated with New Excitatory Spine Synapses on Rat Dentate Granule Cells. Learning 83 Memory, 3:218-228.

Tse, D., Langston, R. F., Kakeyama, M., Bethus, I., Spooner, P. A., Wood, E. R., Witter, M. P., and Morris, R. G. M. (2007). Schemas and memory consolidation. Science, 316(5821):76-82.

Tulving, E. (1992). Memory systems and the brain. Clinical Neuropharmacology, 15(Suppl 1 Pt A):327-328.

Tulving, E., Schacter, D. L., McLachlan, D. R., and Moscovitch, M. (1988). Priming of semantic autobiographical knowledge: a case study of retrograde amnesia. Brain and cognition, 8(1):3-20.

Uylings, H. and Van Eden, C. (1990). Qualitative and quantitative comparison of the prefrontal cortex in rat and in primates, including humans. In Uylings, H. B., Van Eden, C., De Bruin, J., Corner, M., and Feenstra, M., editors, The prefrontal cortex: its structure, function and pathology., pages 31-62. Elsevier, Amsterdam.

Uylings, H. B., Groenewegen, H. J., and Kolb, B. (2003). Do rats have a prefrontal cortex? Behavioural Brain Research, 146(1-2):3-17.

Valenstein, E., Bowers, D., Verfaellie, M., Heilman, K. M., Day, A., and Watson, R. T. (1987). Retrosplenial amnesia. Brain, 110:1631-46.

Van Groen, T. and Wyss, J. M. (2003). Connections of the retrosplenial granular b cortex in the rat. The Journal of comparative neurology, 463(3):249-63.

van Praag, H., Kempermann, G., and Gage, F. H. (2000). Neural consequences of environmental enrichment. Nature reviews. Neuroscience, 1(3):191-8.

Vann, S. D., Aggleton, J. P., and Maguire, E. A. (2009). What does the retrosplenial cortex do? Nature reviews. Neuroscience, 10(11):792-802.

Vann, S. D., Brown, M. W., Erichsen, J. T., and Aggleton, J. P. (2000). Fos imaging reveals differential patterns of hippocampal and parahippocampal subfield activation in rats in response to different spatial memory tests. The Journal of neuroscience, 20(7):2711-8.

Vetere, G., Restivo, L., and Ammassari-Teule, M. (2012). Pre-synaptic control of remote fear extinction in the neocortex. Frontiers in behavioral neuroscience, 6:1-6. 
Vetere, G., Restivo, L., Cole, C. J., Ross, P. J., Ammassari-Teule, M., Josselyn, S. a., and Frankland, P. W. (2011). Spine growth in the anterior cingulate cortex is necessary for the consolidation of contextual fear memory. Proceedings of the National Academy of Sciences of the United States of America, 108(20):8456-60.

Victor, M. and Agamanolis, D. (1990). Amnesia due to Lesions Confined to the Hippocampus: A Clinical-Pathologic Study. Journal of cognitive neuroscience, $2(3): 246-57$.

Viskontas, I. V., McAndrews, M. P., and Moscovitch, M. (2000). Remote episodic memory deficits in patients with unilateral temporal lobe epilepsy and excisions. The Journal of neuroscience, 20(15):5853-7.

Vnek, N. and Rothblat, L. (1993). Rats with hippocampal damage demonstrate retrograde amnesia for object discrimination. Society for Neuroscience Abstract, 19:363.

Vogt, B. A., Finch, D. M., and Olson, C. R. (1992). Functional heterogeneity in cingulate cortex: the anterior executive and posterior evaluative regions. Cerebral cortex, 2(6):435-43.

Vogt, B. a. and Peters, a. (1981). Form and distribution of neurons in rat cingulate cortex: areas 32, 24, and 29. The Journal of comparative neurology, 195(4):603-25.

Vogt, B. A., Vogt, L., and Farber, N. B. (2004). Cingulate cortex and disease models. In Paxinos, G., editor, The rat nervous system, pages 705-727. Academic Press, 3rd edition.

Volk, L. J., Bachman, J. L., Johnson, R., Yu, Y., and Huganir, R. L. (2013). PKM- $\zeta$ is not required for hippocampal synaptic plasticity, learning and memory. Nature, 493(7432):420-3.

von Kraus, L. M., Sacktor, T. C., and Francis, J. T. (2010). Erasing sensorimotor memories via PKMzeta inhibition. PloS one, 5(6):e11125.

Wall, V. L., Fischer, E. K., and Bland, S. T. (2012). Isolation rearing attenuates social interaction-induced expression of immediate early gene protein products in the medial prefrontal cortex of male and female rats. Physiology Es Behavior, 107(3):440-450.

Wan, H., Aggleton, J. P., and Brown, M. W. (1999). Different contributions of the hippocampus and perirhinal cortex to recognition memory. The Journal of neuroscience : the official journal of the Society for Neuroscience, 19(3):11428.

Wang, G.-w. and Cai, J.-x. (2008). Reversible disconnection of the hippocampalprelimbic cortical circuit impairs spatial learning but not passive avoidance learning in rats. Neurobiology of learning and memory, 90:365-373. 
Wang, H., Hu, Y., and Tsien, J. Z. (2006). Molecular and systems mechanisms of memory consolidation and storage. Progress in neurobiology, 79(3):123-35.

Wang, J., Aigner, T., and Mishkin, M. (1990). Effects of neostriatal lesions on visual habit formation in rhesus monkeys. In Society for Neuroscience Abstract.

Wang, S. and Redgrave, P. (1997). Microinjections of muscimol into lateral superior colliculus disrupt orienting and oral movements in the formalin model of pain. Neuroscience, 81(4):967-88.

Wang, S.-H., Tse, D., and Morris, R. G. M. (2012). Anterior cingulate cortex in schema assimilation and expression. Learning \& memory, 19(8):315-8.

Ward, M. T., Oler, J. A., and Markus, E. J. (1999). Hippocampal dysfunction during aging I: deficits in memory consolidation. Neurobiology of aging, 20(4):363-72.

Warrington, E. and Weiskrantz, L. (1968). New method of testing long-term retention with special reference to amnesic patients. Nature, 217:972-974.

Warrington, E. and Weiskrantz, L. (1974). The effect of prior learning on subsequent retention in amnesic patients. Neuropsychologia, 12:419-428.

Warrington, E. K. and McCarthy, R. A. (1988). The fractionation of retrograde amnesia. Brain and cognition, 7(2):184-200.

Wartman, B. C. and Holahan, M. R. (2013). The use of sequential hippocampaldependent and -non-dependent tasks to study the activation profile of the anterior cingulate cortex during recent and remote memory tests. Neurobiology of learning and memory, 106:334-42.

Weible, A. P. (2013). Remembering to attend: The anterior cingulate cortex and remote memory. Behavioural Brain Research, 245:63-75.

Weible, A. P., Rowland, D. C., Monaghan, C. K., Wolfgang, N. T., and Kentros, C. G. (2012). Neural correlates of long-term object memory in the mouse anterior cingulate cortex. The Journal of neuroscience, 32(16):5598-608.

Weisand, M. P., Astur, R. S., and Sutherland, R. J. (1996). The specificity and temporal characteristics of retrograde amnesia after hippocampal lesions. In Society for Neuroscience Abstract, page 1118.

Weissman, D. H., Gopalakrishnan, A., Hazlett, C. J., and Woldorff, M. G. (2005). Dorsal anterior cingulate cortex resolves conflict from distracting stimuli by boosting attention toward relevant events. Cerebral Cortex, 15:229237.

Whishaw, I. Q., McKenna, J. E., and Maaswinkel, H. (1997). Hippocampal lesions and path integration. Current opinion in neurobiology, 7(2):228-34. 
White, N. M. and McDonald, R. J. (2002). Multiple parallel memory systems in the brain of the rat. Neurobiology of learning and memory, 77(2):125-84.

Wiltgen, B. J., Brown, R. a. M., Talton, L. E., and Silva, A. J. (2004). New circuits for old memories: the role of the neocortex in consolidation. Neuron, 44(1):101-8.

Wiltgen, B. J., Sanders, M. J., Anagnostaras, S. G., Sage, J. R., and Fanselow, M. S. (2006). Context fear learning in the absence of the hippocampus. The Journal of neuroscience, 26(20):5484-91.

Wiltgen, B. J., Zhou, M., Cai, Y., Balaji, J., Karlsson, M. G., Parivash, S. N., Li, W., and Silva, A. J. (2010). The hippocampus plays a selective role in the retrieval of detailed contextual memories. Current biology, 20(15):1336-44.

Winocur, G. (1982). Radial-arm-maze behavior by rats with dorsal hippocampal lesions: effect of cuing. Journal of comparative and physiological psychology, 96(2):155-69.

Winocur, G. (1990). Anterograde and retrograde amnesia in rats with dorsal hippocampal or dorsomedial thalamic lesions. Behavioural brain research, $38(2): 145-54$.

Winocur, G., McDonald, R. M., and Moscovitch, M. (2001). Anterograde and retrograde amnesia in rats with large hippocampal lesions. Hippocampus, $11(1): 18-26$.

Winocur, G., Moscovitch, M., and Bontempi, B. (2010). Memory formation and long-term retention in humans and animals: convergence towards a transformation account of hippocampal-neocortical interactions. Neuropsychologia, 48(8):2339-2356.

Winocur, G., Moscovitch, M., Caruana, D. a., and Binns, M. a. (2005a). Retrograde amnesia in rats with lesions to the hippocampus on a test of spatial memory. Neuropsychologia, 43(11):1580-90.

Winocur, G., Moscovitch, M., Fogel, S., Rosenbaum, R. S., and Sekeres, M. (2005b). Preserved spatial memory after hippocampal lesions: effects of extensive experience in a complex environment. Nature neuroscience, 8(3):2735 .

Witter, M. P. (1993). Organization of the entorhinal-hippocampal system: a review of current anatomical data. Hippocampus, 3:33-44.

Witter, M. P. (1997). No Entorhinal cortex of the rat: cytoarchitectonic subdivisions and the origin and distribution of cortical efferents. Hippocampus, $7: 146-183$.

Witter, M. P., Griffioen, A. W., Jorritsma-Byham, B., and Krijnen, J. L. M. (1988). Entorhinal projections to the hippocampal CA1 region in the rat: an underestimated pathway. Neuroscience letters, 85:193-198. 
Witter, M. P., Ostendorf, R. H., and Groenewegen, H. J. (1990). Heterogeneity in the dorsal subiculum of the rat: distinct neuronal zones project to different cortical and subcortical targets. European Journal of Neuroscience, 2:718725 .

Wolfer, D. P., Madani, R., Valenti, P., and Lipp, H. P. (2001). Extended analysis of path data from mutant mice using the public domain software Wintrack. Physiology $\& 3$ behavior, 73(5):745-753.

Woodard, J. L., Seidenberg, M., Nielson, K. A., Miller, S. K., Franczak, M., Antuono, P., Douville, K. L., and Rao, S. M. (2007). Response to Memories of Different Ages. Journal of cognitive neuroscience, 19(7):1113-1124.

Xu, T., Yu, X., Perlik, A. J., Tobin, W. F., Zweig, J. a., Tennant, K., Jones, T., and Zuo, Y. (2009). Rapid formation and selective stabilization of synapses for enduring motor memories. Nature, 462(7275):915-9.

Yang, G., Pan, F., and Gan, W.-b. (2009). Stably maintained dendritic spines are associated with lifelong memories. Nature, 462(7275):920-924.

Yao, Y., Kelly, M. T., Sajikumar, S., Serrano, P., Tian, D., Bergold, P. J., Frey, J. U., and Sacktor, T. C. (2008). PKM zeta maintains late long-term potentiation by N-ethylmaleimide-sensitive factor/GluR2-dependent trafficking of postsynaptic AMPA receptors. The Journal of neuroscience, 28(31):7820-7.

Yin, Y., Edelman, G. M., and Vanderklish, P. W. (2002). The brain-derived neurotrophic factor enhances synthesis of Arc in synaptoneurosomes. Proceedings of the National Academy of Sciences of the United States of America, 99(4):2368-73.

Young, E., Cesena, T., Meiri, K. F., and Perrone-Bizzozero, N. I. (2002). Changes in protein kinase $\mathrm{C}$ (PKC) activity, isozyme translocation, and GAP43 phosphorylation in the tat hippocampal formation after a single-trial contextual fear conditioning paradigm. Hippocampus, 12:457-464.

Young, E. A., Owen, E. H., Meiri, K. F., and Wehner, J. M. (2000). Alterations in hippocampal GAP-43 phosphorylation and protein level following contextual fear conditioning. Brain research, 860:95-103.

Zaehle, T., Jordan, K., Wüstenberg, T., Baudewig, J., Dechent, P., and Mast, F. W. (2007). The neural basis of the egocentric and allocentric spatial frame of reference. Brain research, 1137(1):92-103.

Zehle, S., Bock J, Jezierski, G., Gruss, M., and Braun, K. (2007). Methylphenidate Treatment Recovers Stress-Induced Elevated Dendritic Spine Densities in the Rodent Dorsal Anterior Cingulate Cortex. Developmental neurobiology, 67:1891-1900. 
Zhang, Y., Fukushima, H., and Kida, S. (2011). Induction and requirement of gene expression in the anterior cingulate cortex and medial prefrontal cortex for the consolidation of inhibitory avoidance memory. Molecular Brain, 4(1):4.

Zhou, Q., Homma, K. J., and Poo, M.-m. (2004). Shrinkage of dendritic spines associated with long-term depression of hippocampal synapses. Neuron, 44(5):749-57.

Zhu, X. O., McCabe, B. J., Aggleton, J. P., and Brown, M. W. (1997). Differential activation of the rat hippocampus and perirhinal cortex by novel visual stimuli and a novel environment. Neuroscience letters, 229(2):141-3.

Zola-Morgan, S. and Squire, L. R. (1990). The primate hippocampal formation: Evidence for a time-limited role in memory storage. Science, 250:288-290.

Zola-Morgan, S., Squire, L. R., and Amaral, D. G. (1986). Human amnesia and the medial temporal region: enduring memory impairment following a bilateral lesion limited to field CA1 of the hippocampus. The Journal of neuroscience, 6(10):2950-67.

Zuber, M. X., Strittmatter, S. M., and Fishman, M. C. (1989). A membranetargeting signal in the amino terminus of the neuronal protein GAP-43. $\mathrm{Na}$ ture, 341(6240):345-8. 


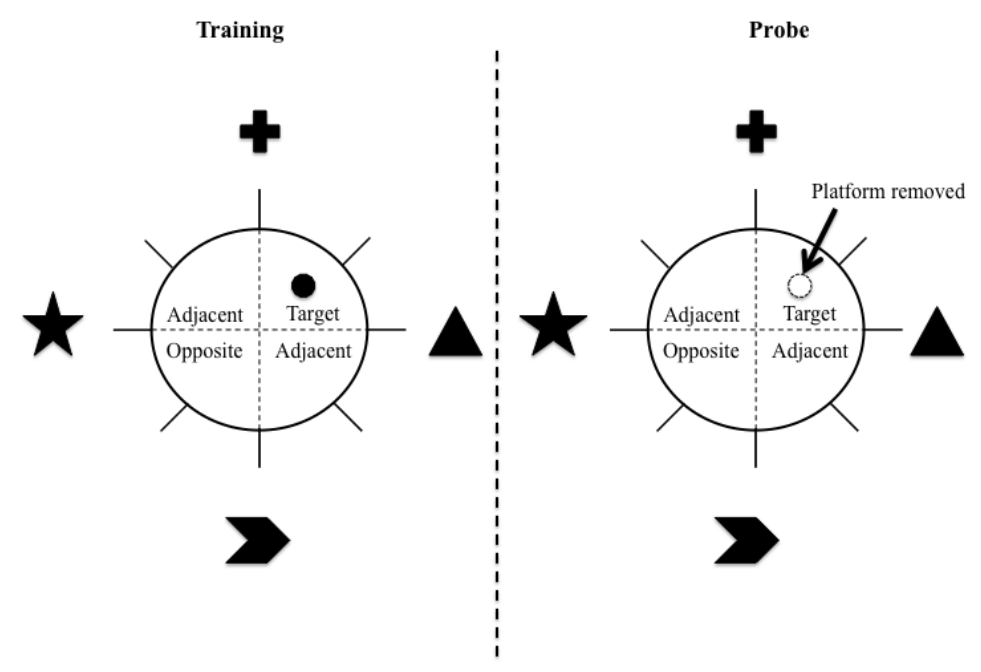

Figure 1.2: Illustration of the water maze task. 


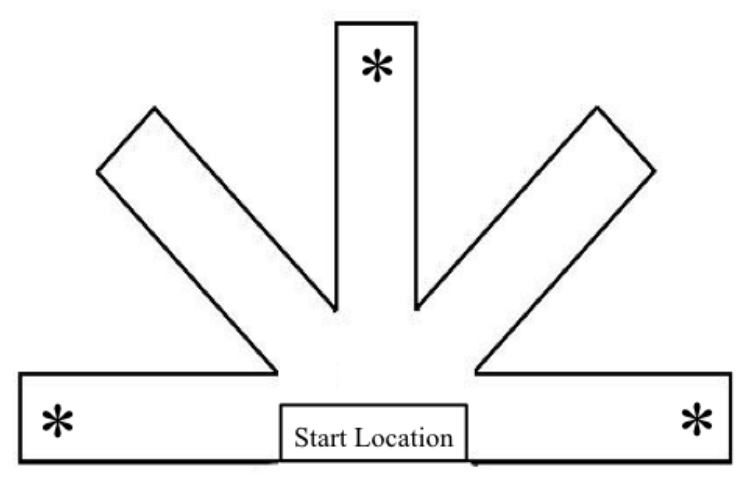

Figure 1.3: Illustration of the radial arm maze task. 


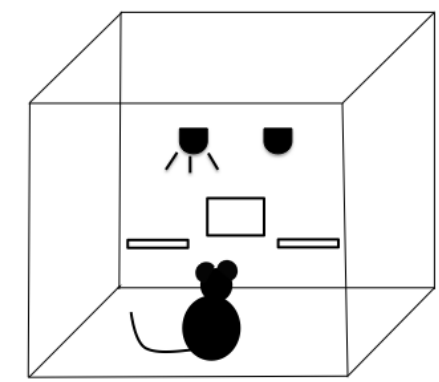

Figure 1.4: Illustration of the operant task. 


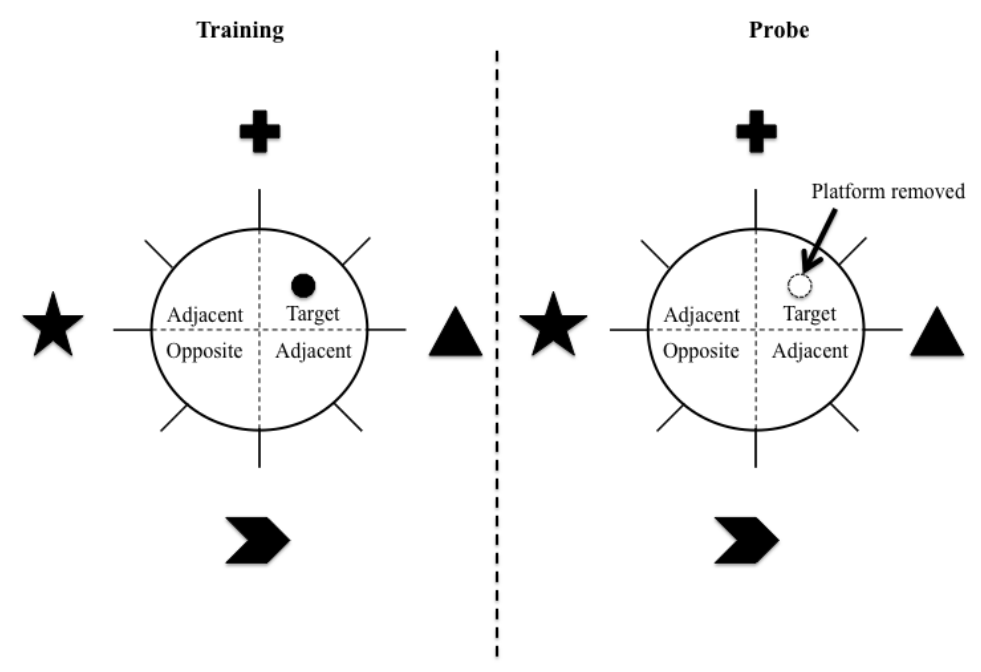

Figure 1.2: Illustration of the water maze task. 


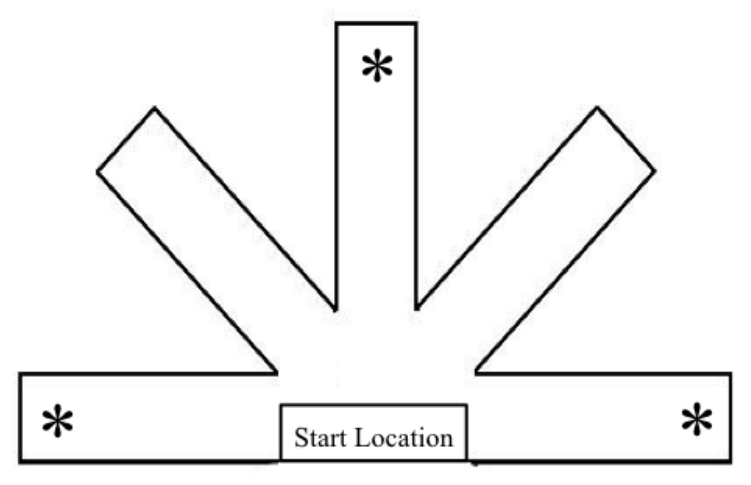

Figure 1.3: Illustration of the radial arm maze task. 


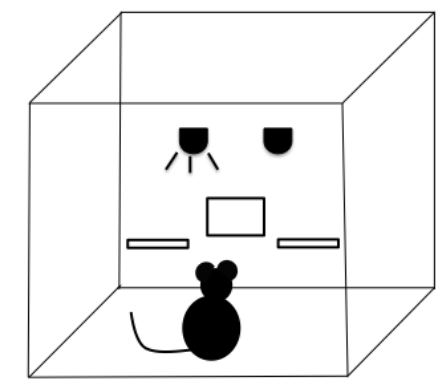

Figure 1.4: Illustration of the operant task. 